\title{
Technical Basis for User Interface Design of Health IT
}

\author{
Michael E. Wiklund \\ Jonathan Kendler \\ Limor Hochberg \\ $U L, L L C$ \\ Matthew B. Weinger \\ Vanderbilt University
}

This publication is available free of charge from:

http://dx.doi.org/10.6028/NIST.GCR.15-996

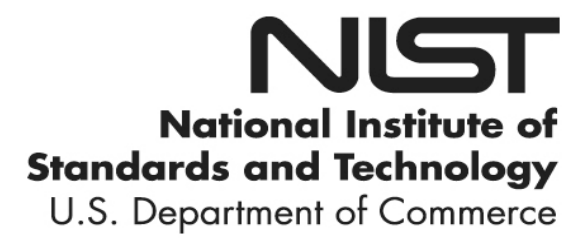




\title{
Technical Basis for User Interface Design of Health IT
}

\author{
Prepared for \\ U.S. Department of Commerce \\ Information Technology Laboratory \\ National Institute of Standards and Technology \\ Gaithersburg, MD 20899
}

Michael E. Wiklund

Jonathan Kendler

Limor Hochberg

$U L L L C$

Matthew B. Weinger

Vanderbilt University Medical Center

This publication is available free of charge from:

http://dx.doi.org/10.6028/NIST.GCR.15-996

September 2015

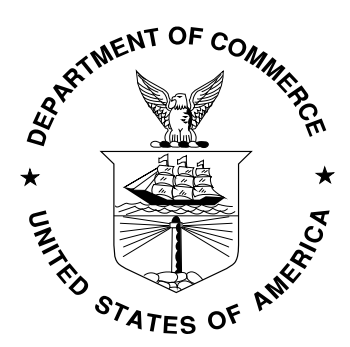

U.S. Department of Commerce

Penny Pritzker, Secretary

National Institute of Standards and Technology Willie May, Under Secretary of Commerce for Standards and Technology and Director 
This publication was produced as part of contract SB1341-11-CN-0011 with the National Institute of Standards and Technology. The contents of this publication do not necessarily reflect the views or policies of the National Institute of Standards and Technology or the US Government. 


\section{Table of Contents}

1 Introduction

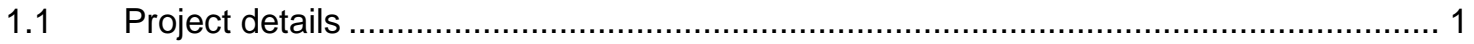

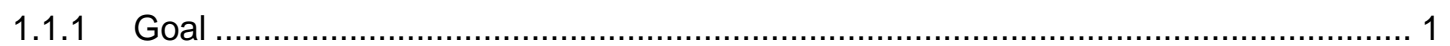

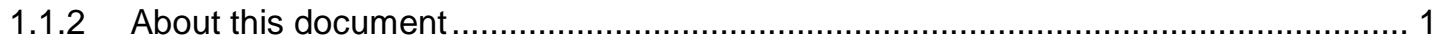

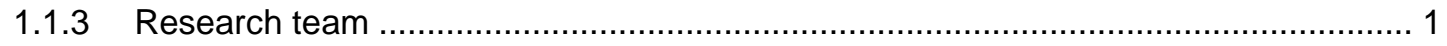

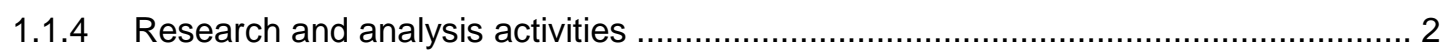

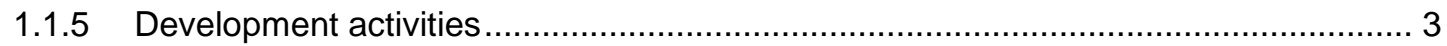

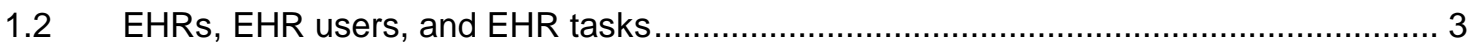

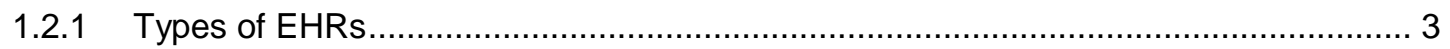

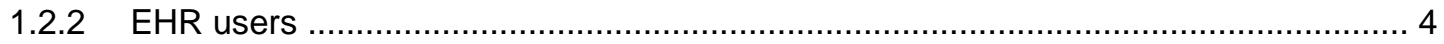

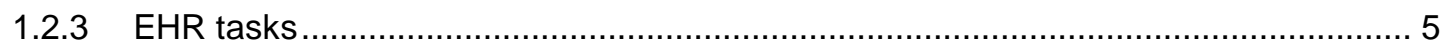

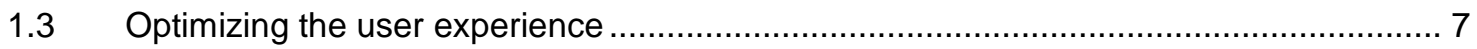

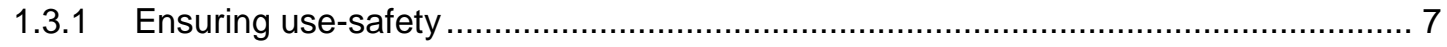

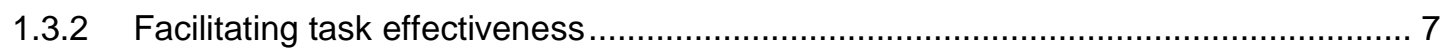

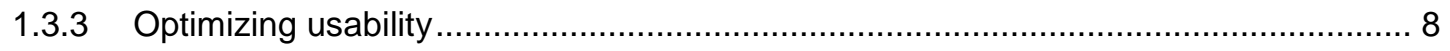

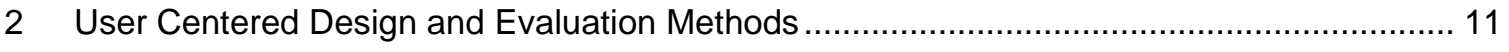

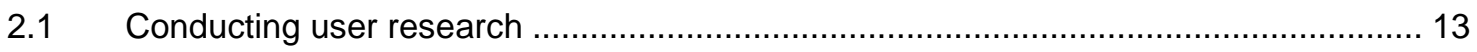

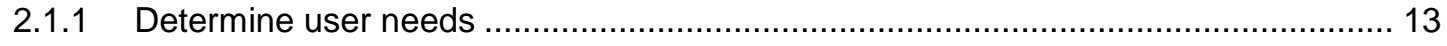

2.1.2 Identify work environment characteristics....................................................... 16

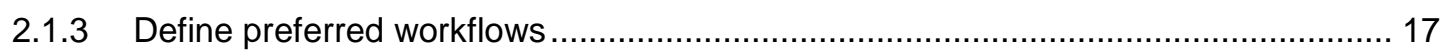

2.1.4 Determine and evaluate common workflows ....................................................... 17

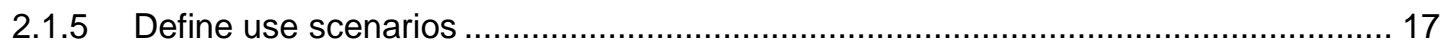

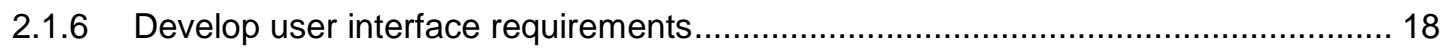

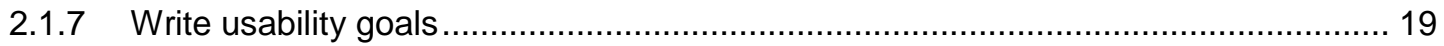

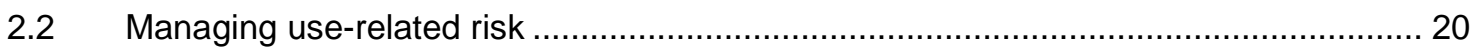

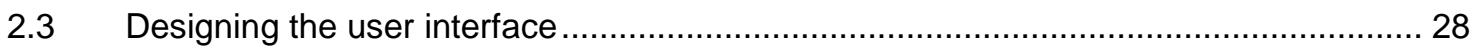

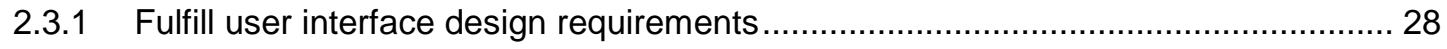

2.3.2 Re-analyze tasks and perform additional risk management .................................. 29

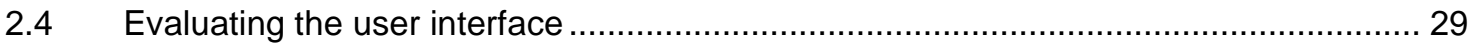

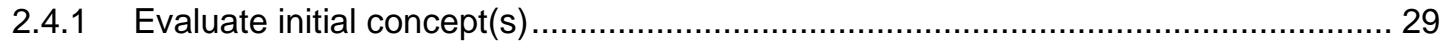

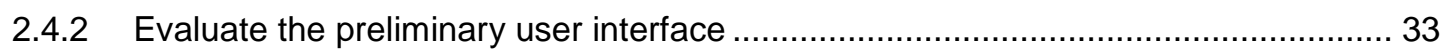

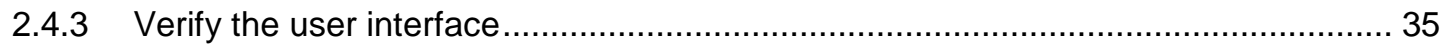

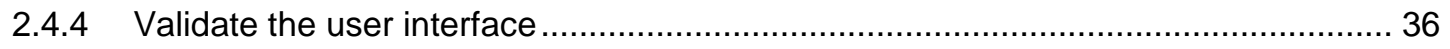

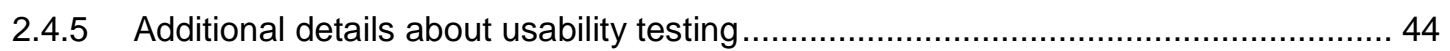

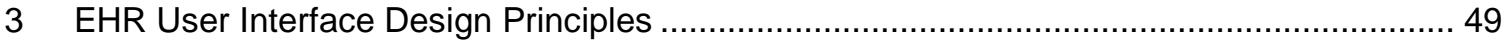




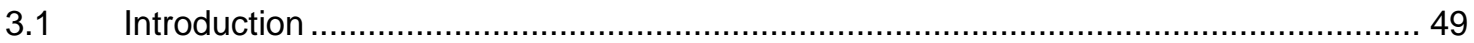

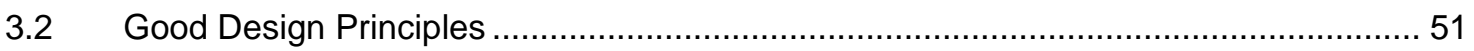

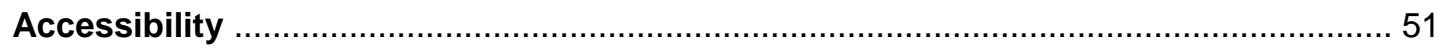

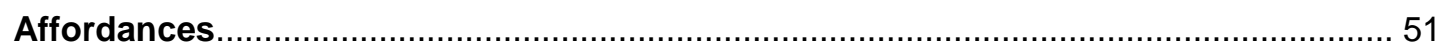

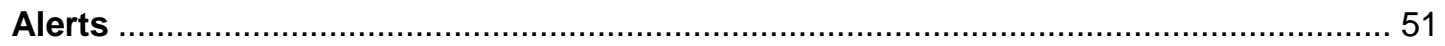

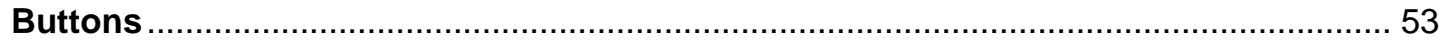

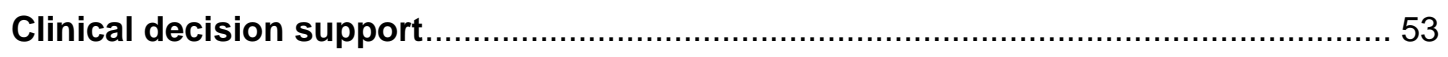

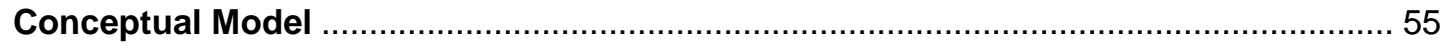

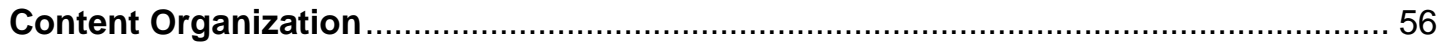

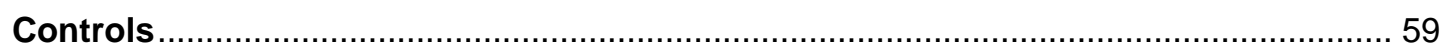

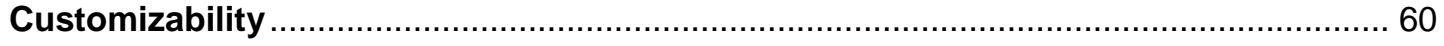

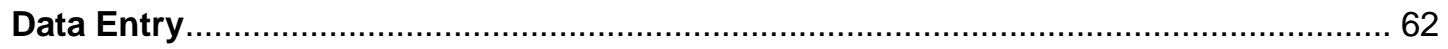

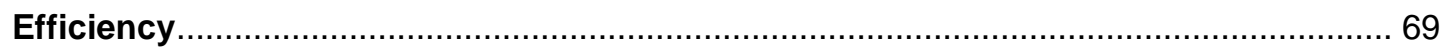

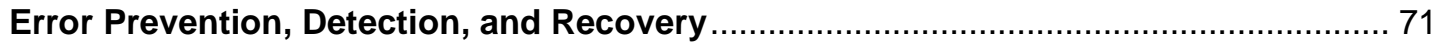

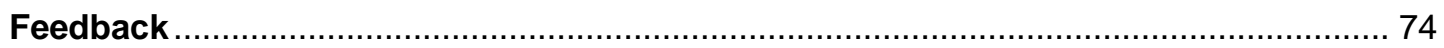

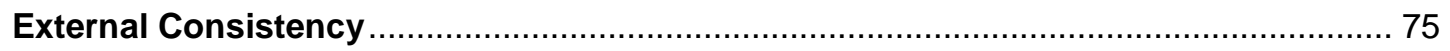

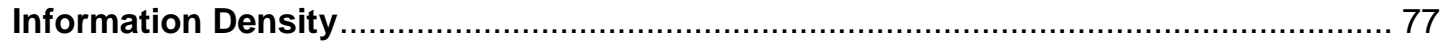

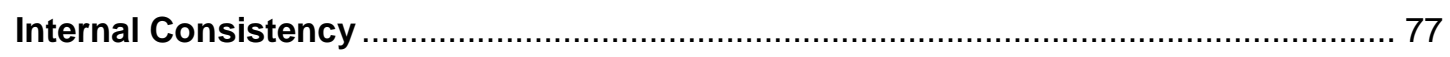

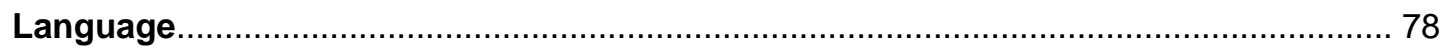

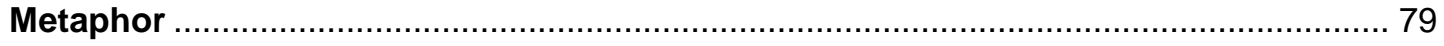

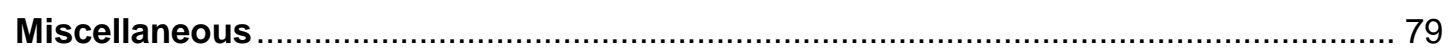

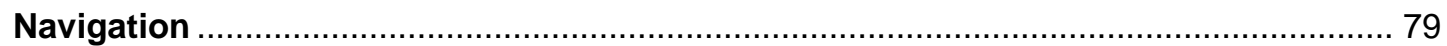

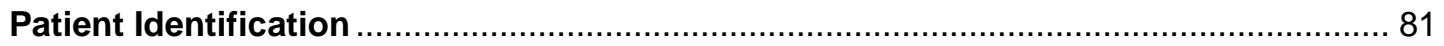

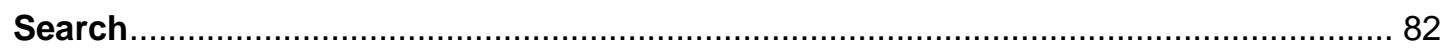

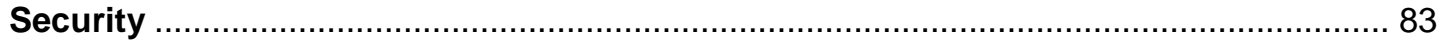

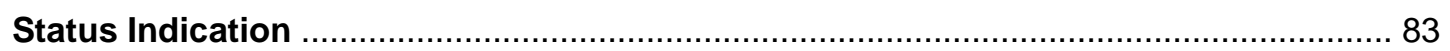

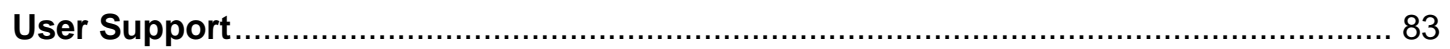

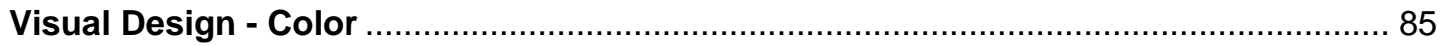

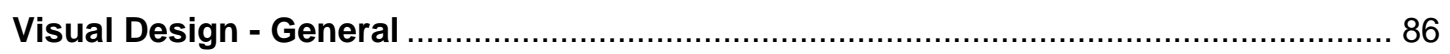

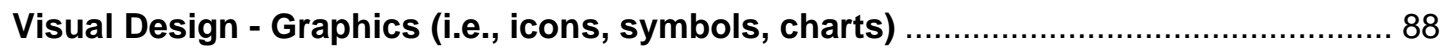

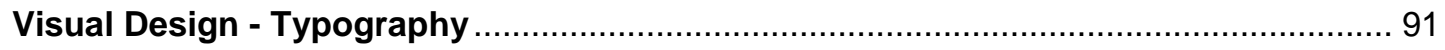

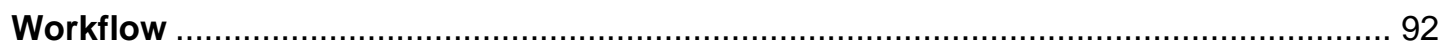

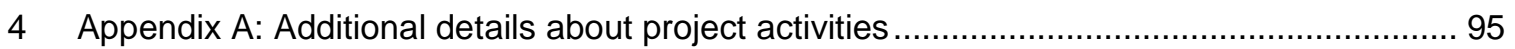

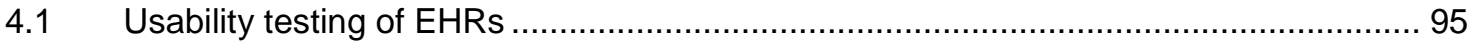

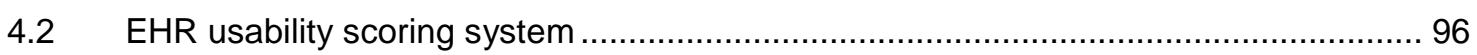

4.2.1 EHRUS usability scoring system development …............................................ 98

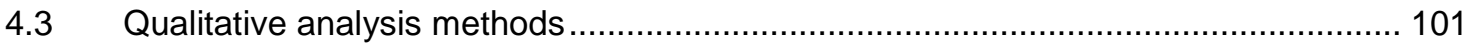

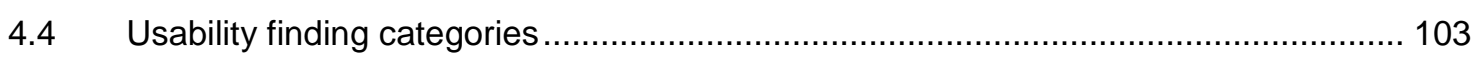

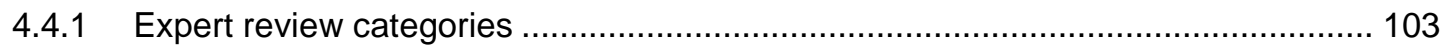




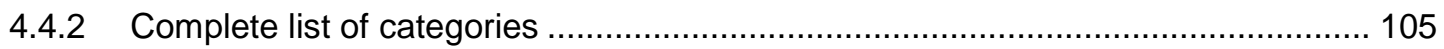

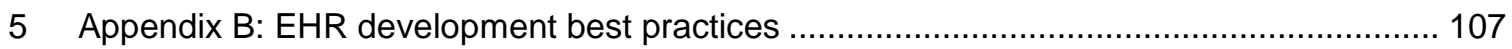

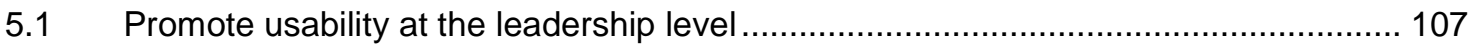

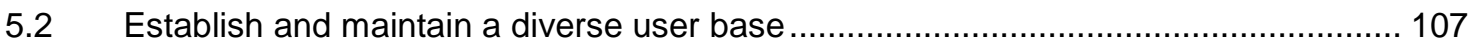

5.3 Conduct a variety of user-centered design activities.............................................. 108

$5.4 \quad$ Conduct formative usability tests early and often ................................................ 108

5.5 Invest in professional usability expertise and encourage frequent interaction between

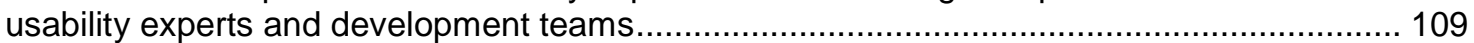

5.6 Integrate user-centered design into the development process ................................. 109

$5.7 \quad$ Infuse design and evaluation with clinical expertise ............................................. 109

5.8 Develop a use-related risk management process ................................................. 110

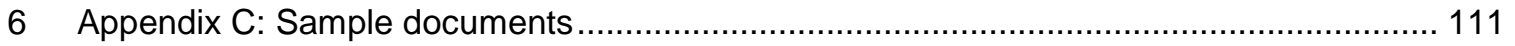

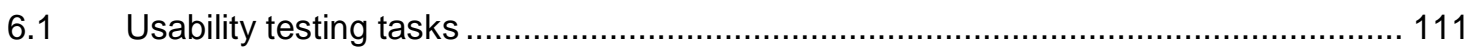

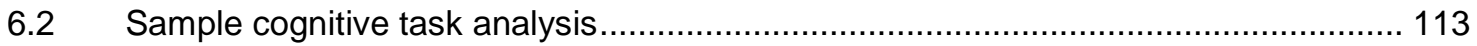

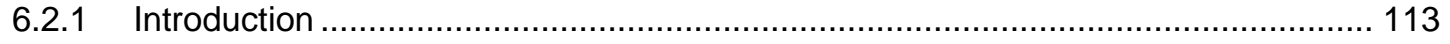

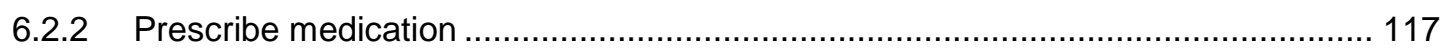

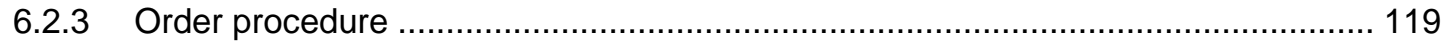

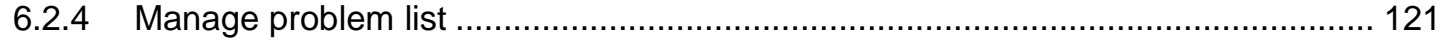

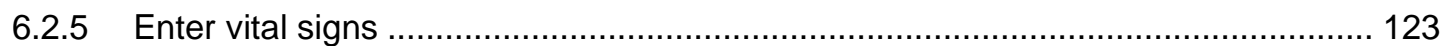

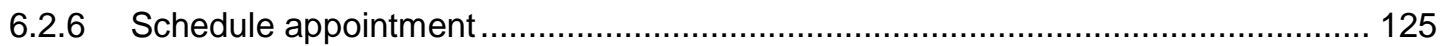

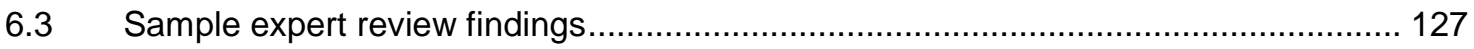

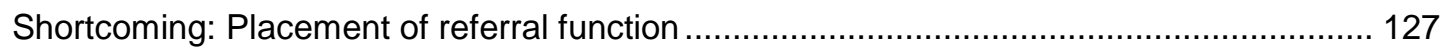

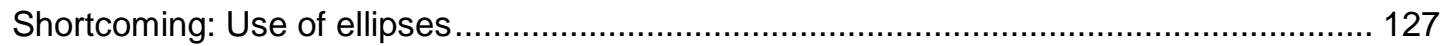

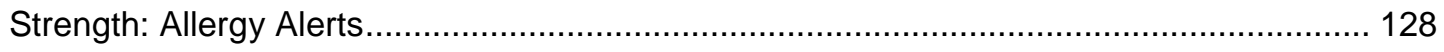

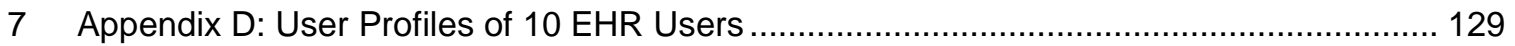

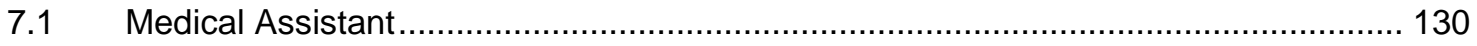

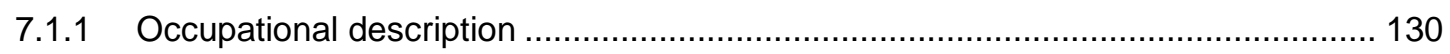

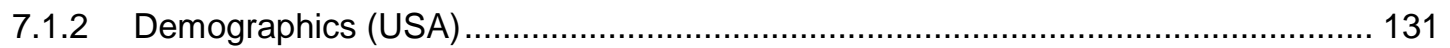

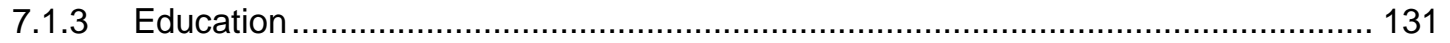

7.1.4 Electronic Health Record (EHR) Use environment(s) ........................................ 132

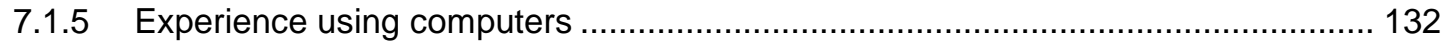

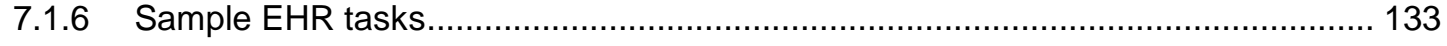

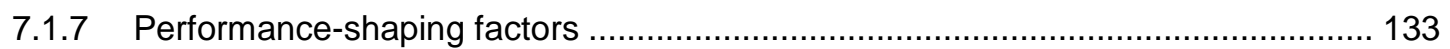

7.2 Medical Record and Health Information Technicians ............................................... 134

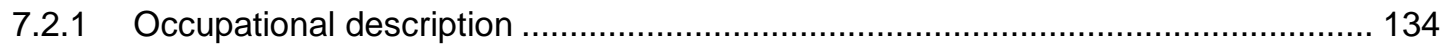

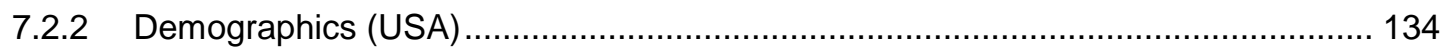

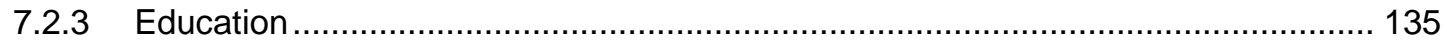

7.2.4 Electronic Health Record (EHR) Use environment(s) ...................................... 135

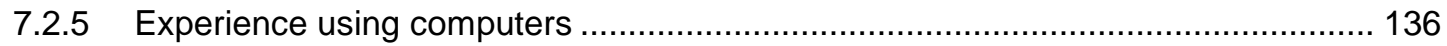




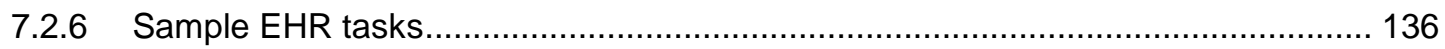

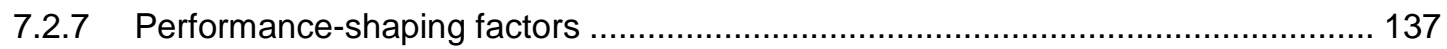

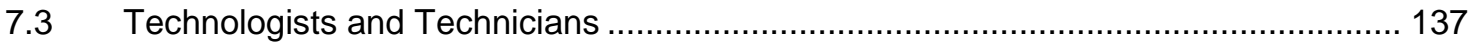

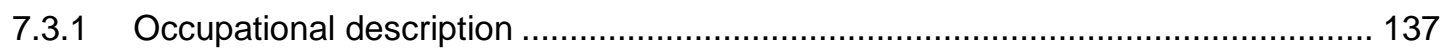

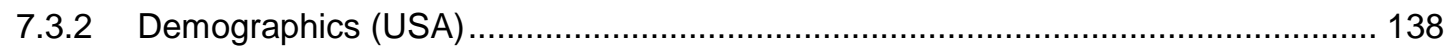

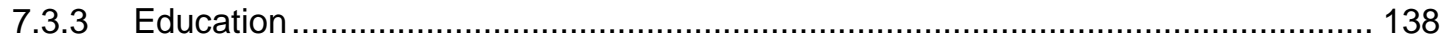

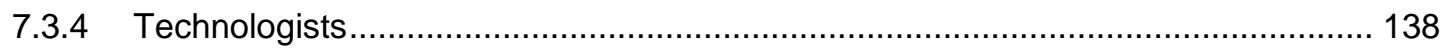

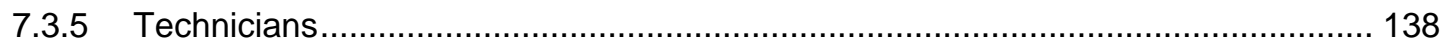

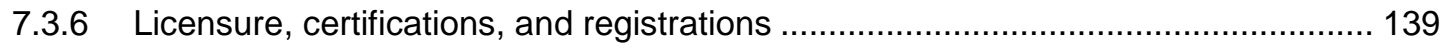

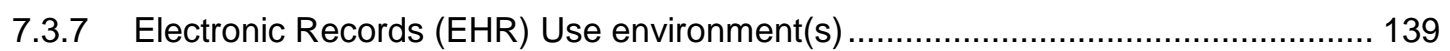

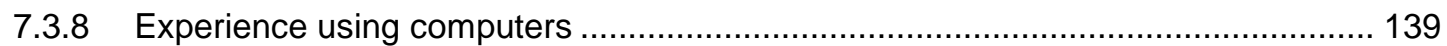

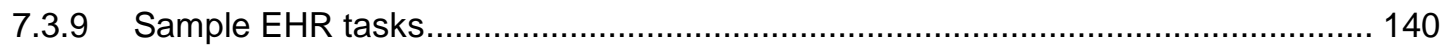

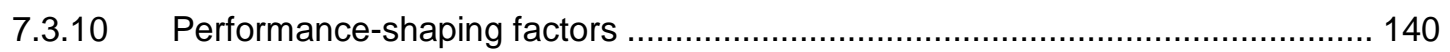

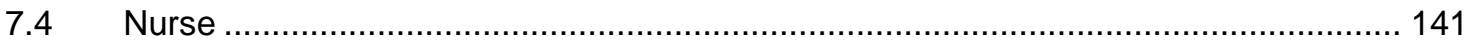

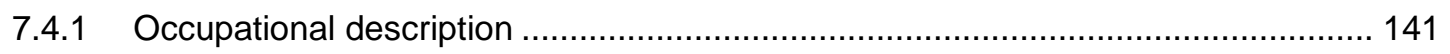

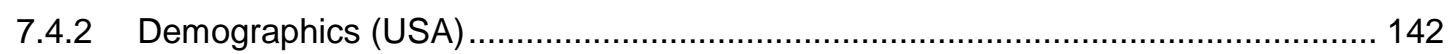

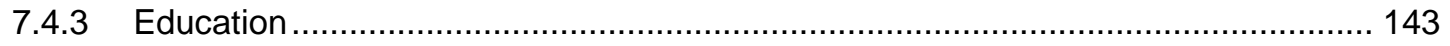

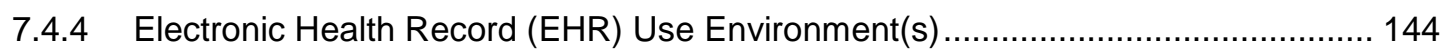

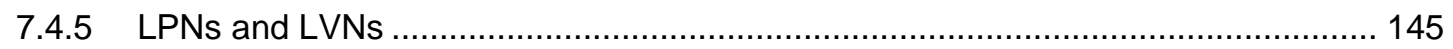

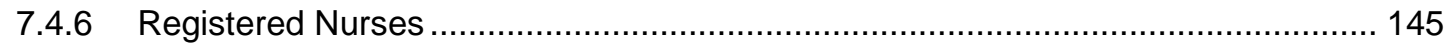

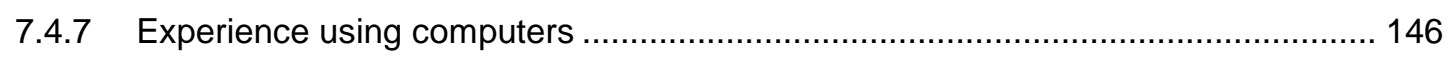

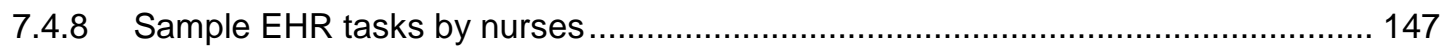

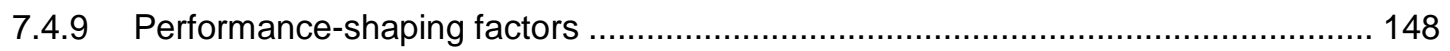

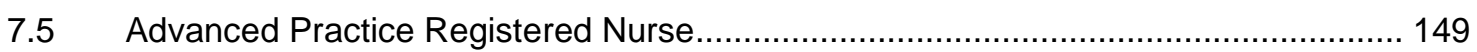

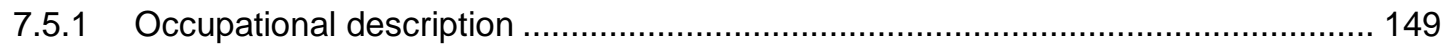

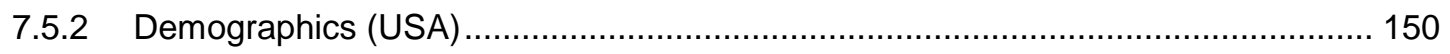

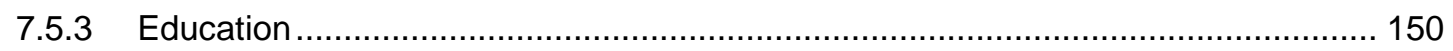

7.5.4 Electronic Health Record (EHR) Use environment(s) ..................................... 150

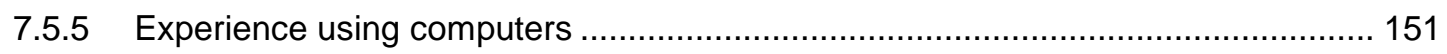

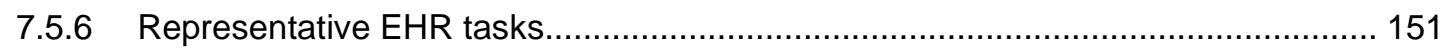

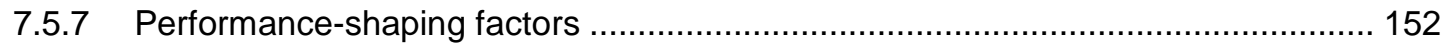

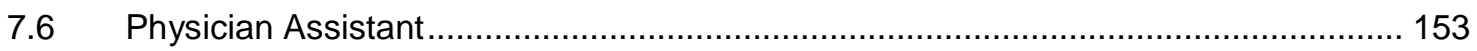

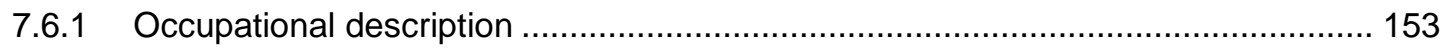

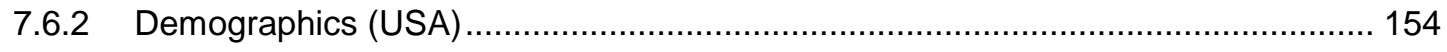

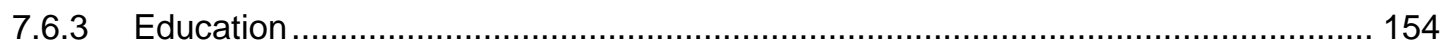

7.6.4 Electronic Health Record (EHR) Use environment(s) ..................................... 155

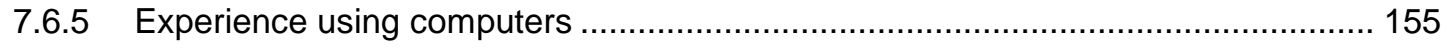

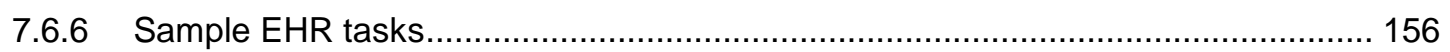

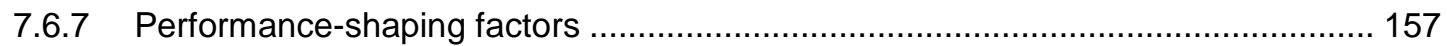




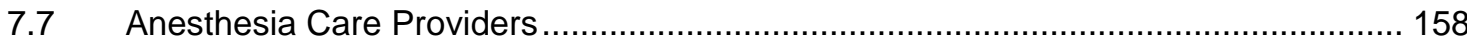

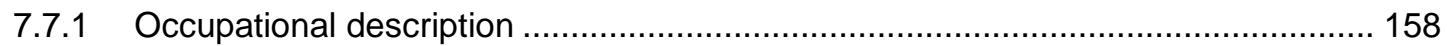

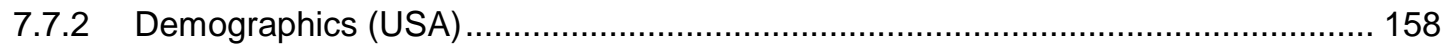

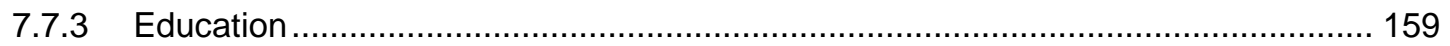

7.7.4 Electronic Health Record (EHR) Use environment(s) ........................................ 159

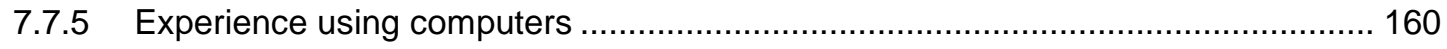

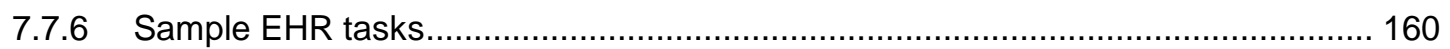

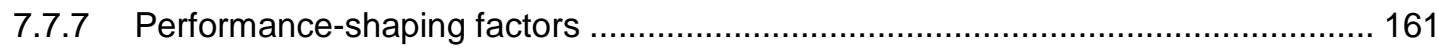

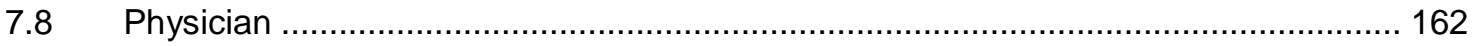

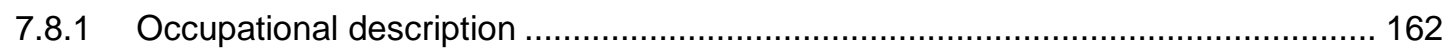

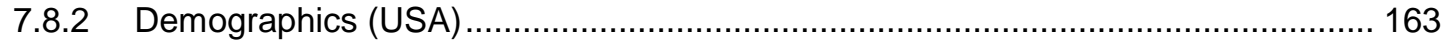

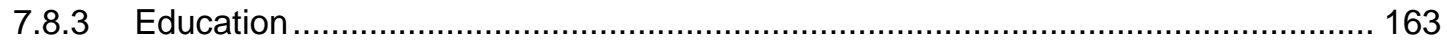

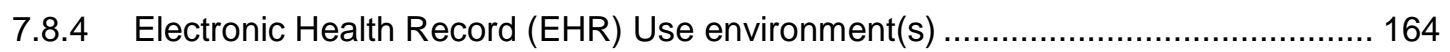

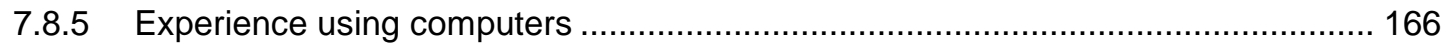

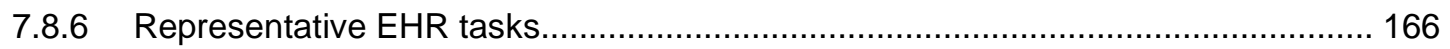

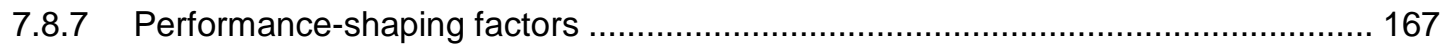

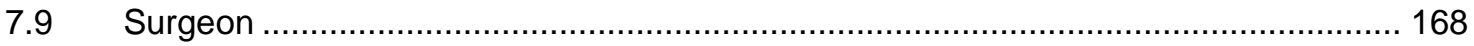

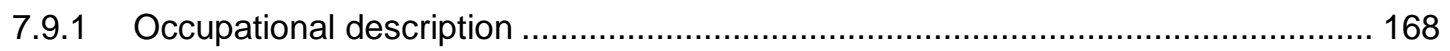

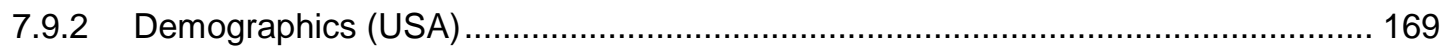

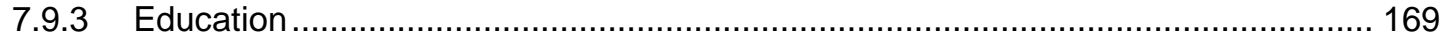

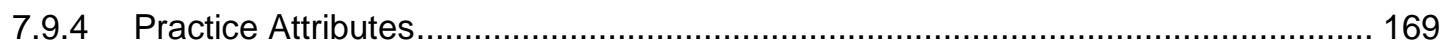

7.9.5 Electronic Health Record (EHR) Use environment(s) ....................................... 169

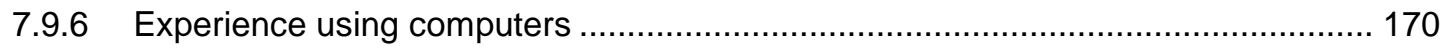

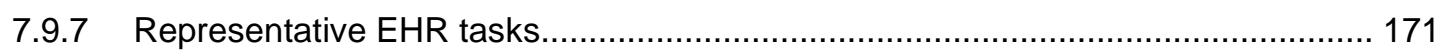

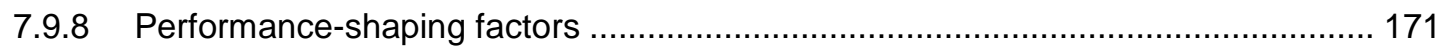

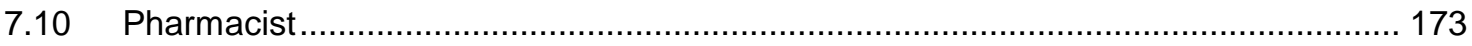

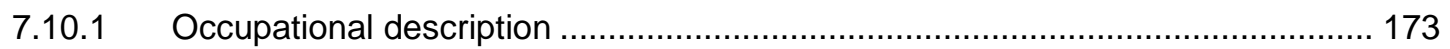

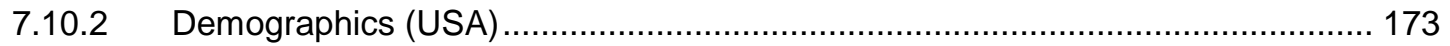

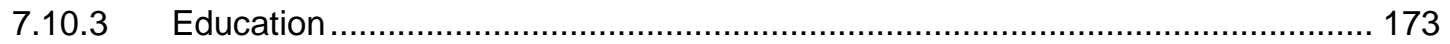

7.10.4 Electronic Health Record (EHR) Use environment(s) ................................... 173

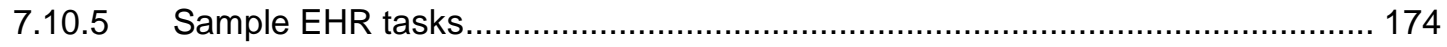

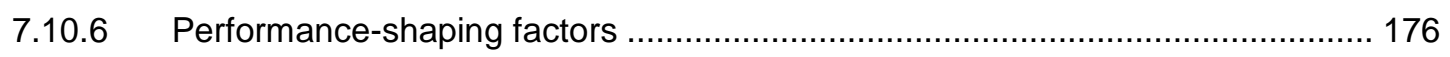

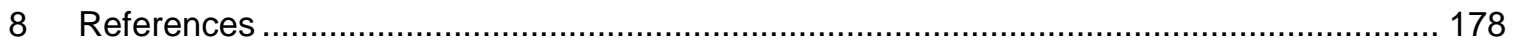




\section{List of Tables}

Table 1-1: User interface characteristics that can lead to user satisfaction or dissatisfaction........ 8

Table 2-1: Sample objective and subjective usability goals.

Table 2-2: Sample possible harms and use errors, which may be derived from a fault tree or similar analysis.

Table 2-3: Levels of probability and severity associated with use errors and their potential harms (adapted from ISO 14971:2007 Medical devices -- Application of risk management to medical devices).

Table 2-4: Sample guidelines and their intended use-safety benefit. ..................................... 26

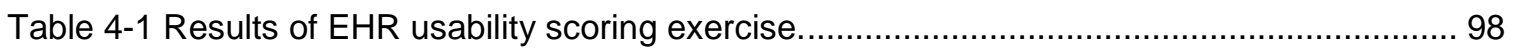

Table 4-2: Number of findings generated from each data source.......................................... 102

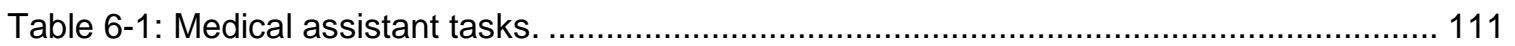

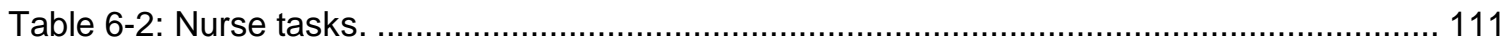

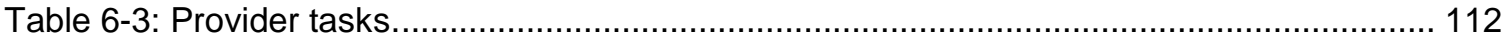

Table 6-4: Cognitive task analysis insights and resulting design solutions.............................. 114

\section{List of Figures}

Figure 2-1: An illustration of a sample implementation of a user-centered design process.......... 12

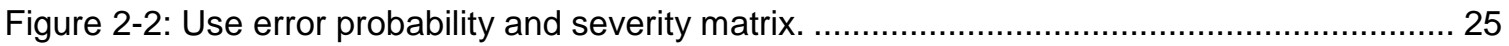

Figure 2-3: An illustration of the risk analysis and mitigation process...................................... 39

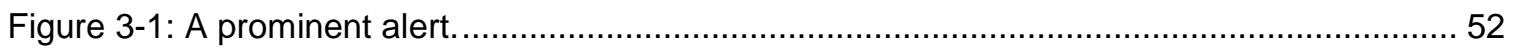

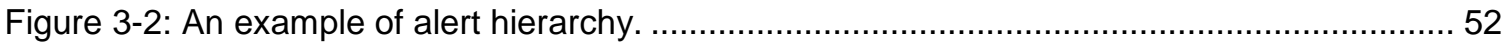

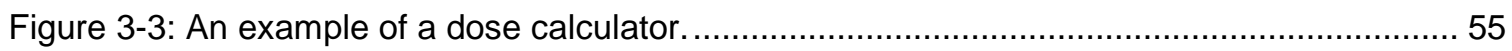

Figure 3-4: A sample patient summary that presents five groupings of related patient information.

Figure 3-5: Clicking on a table header sorts the table's data according to selected column. ....... 57

Figure 3-6: Samples of scrolling controls for tabular data..................................................... 58

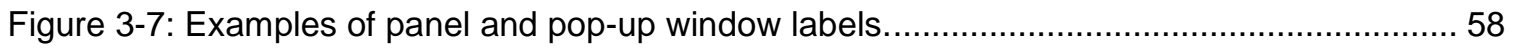

Figure 3-8: Today's current parameters are highlighted with a green header and thick black outline.

Figure 3-9: An example of "Reset" controls that cause the EHR to revert to its default settings.. 60 Figure 3-10: The "View more" buttons provide access to additional details; an example of progressive disclosure.

Figure 3-11: An example of items presented in a vertical list (left) and in a paragraph format

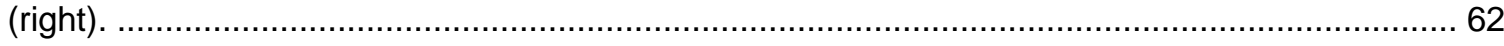

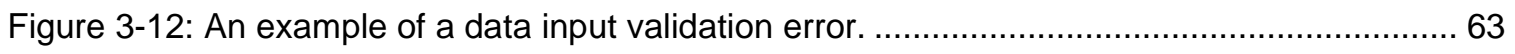

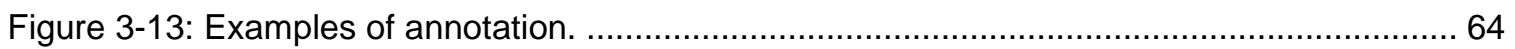

Figure 3-14: Examples of selection controls (left) and text entry fields (right). ............................ 64

Figure 3-15: An example of a conventional QWERT keyboard layout (top) and a less common

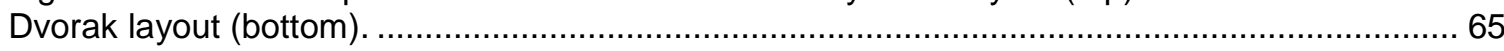

Figure 3-16: Slider controls used to toggle the alarm status and adjust its volume.................... 65 
Figure 3-17: Examples of a well aligned field layout (left) and a haphazardly arranged layout (right).

Figure 3-18: An example of well-sized fields (left) and fields that are too small to contain the expected information (right). 66

Figure 3-19: Sample controls for adjusting weight, height, and temperature units. .................... 67

Figure 3-20: A sample format suggestion appearing below the Birth Date field. ....................... 67

Figure 3-21: An example of a workflow that utilizes the entered postal code to automatically fill the city field.

Figure 3-22: An example of type-ahead functionality that presents drug names including the entered characters

Figure 3-23: An example of a data import progress indicator.

Figure 3-24: A sample patient overview layout.

Figure 3-25: An example of procedural steps that give the user an indication of their progress through the application.

Figure 6-1: Referral function located under Demographics.

Figure 6-2: Indication of allergies is subtle....... 128

Figure 6-3: Clicking on "..." leads to list of allergies or none. 128

Figure 6-4: Clinical reminders 128 


\section{Introduction}

\subsection{Project details}

This document presents the technical guidance from a NIST-sponsored research project titled "Developing a Usability Framework for Healthcare Information Technology".

The project began in September 2010. It was funded under the American Recovery and Reinvestment Act of 2009. The project ended in September 2015.

\subsubsection{Goal}

The project goal was to develop methods for evaluating the usability of healthcare information technology (HIT) and particularly electronic health records systems (EHRs). The project evolved to focus most intensively on EHR use-safety and the effectiveness of such applications from a user interaction standpoint.

\subsubsection{About this document}

This document's contents are intended to help EHR developers plan and implement a usercentered design and evaluation process that ultimately leads to a safe and effective application. Specifically, it provides:

- Methods describing research, analysis, design, and evaluation practices intended to optimize EHR use-safety, effectiveness, and usability (see section 2 - User Centered Design and Evaluation Methods).

- User interface design principles describing design strategies and practices that are likely to prevent common EHR design flaws and establish a basic level of interaction design quality in an EHR (see section 3 - EHR User Interface Design Principles).

- Descriptions of the research and evaluation activities that led to the development of this document (see Appendix A).

- Summaries of EHR development best practices as described by EHR developers (see Appendix B).

- Sample versions of EHR-related usability engineering documents, including usability test tasks, cognitive task analyses, expert review findings, and user profiles (see Appendix C).

\subsubsection{Research team}

UL-Wiklund's Michael Wiklund served as the Project Director. The research team included the following members: 
- UL-Wiklund - Michael Wiklund, Jonathan Kendler, Limor Hochberg, Danielle Heinle, Kayley Wolf, Maureen Mulcare, Timothy McEwen, Robert Stephens, Celine Berger, Victor Versteeg, and Luis Herrera Suazo

- Vanderbilt University - Matthew B. Weinger, Jason Slagle, Carrie Reale, Shilo Anders, Anne Miller, and Rich Holden

- Design Science - Stephen Wilcox, Robin Littlejohn, Logan McCullar, Katie Hansbro, Neil Mejia, Matthew Cavanagh, Angouh Morfaw, and Dana Long

- The Ohio State University - Emily Patterson and Austin Mount-Campbell

The following individuals also contributed to the research efforts:

- Additional staff members working at UL-Wiklund, Vanderbilt University, and Design Science.

- Daniel Hannon, Tufts University, Director - Human Factors Engineering Program

- David Brick, Village Pediatric Cardiology, affiliated with NYU Langone Medical Center

- Bentzi Karsh, Pascale Carayon, and Tosha Wetterneck, University of Wisconsin

- Sandra Prettyman, University of Akron

- Christine Buchanan, Tony Threat, Kevin Johnson, Joshua Peterson, Vanderbilt University

The research team is indebted to many more individuals, including EHR developers and EHR users, who supported the project in the following ways:

- Provided access to sample EHRs and associated training services.

- Provided access to clinical settings where EHRs were currently in use.

- Participated in surveys, observations, interviews, and usability tests.

These individuals' names are withheld in accordance with IRB-approved research protocols and other terms and conditions pertaining to their participation in the research project.

\subsubsection{Research and analysis activities}

Each of the primary research activities focused on determining user interface design features that contribute to or detract from an EHR's use-safety and effectiveness, included the following (in chronological order of performance):

- Survey - Conducted a Web-based survey of 559 individuals who routinely use EHRs installed in multiple clinical environments within a large healthcare system. 
- Observations and interviews - Visited multiple healthcare facilities operated by two large, multi-hospital healthcare systems to observe and interview a total of 86 clinical, non-clinical and administrative staff that routinely used EHRs.

- $\quad$ Supporting analyses - Analyzed EHR tasks in terms of the associated cognitive demands and potential for harmful use errors as well as use errors that could pose a barrier to successful task completion (i.e., effectiveness); reviewed literature and conducted interviews pertaining to best practices among EHR vendors regarding usercentered approaches to application development to ensure an EHR's use-safety and effectiveness; and collected over 900 user interface design principles published in multiple sources, including national and international standards, textbooks, and websites.

- Usability tests - Conducted formative usability tests of five EHRs with 63 representative users, all of whom were clinicians working in patient care.

- Expert reviews - Engaged six human factors engineering experts (members of the research team) to review the same five EHRs evaluated in the usability tests and identify the applications' user interface strengths and weaknesses (i.e., opportunities for improvement).

\subsubsection{Development activities}

The primary development activities, all focused on providing government and industry with better knowledge and tools for ensuring an EHR's use-safety and effectiveness, included the following:

- User profiles - Developed user profiles describing 10 common types of EHR users.

- Vendor best practices - Documented vendor best practices for implementing a usercentered approach to designing and evaluating an EHR user interface.

- Usability scoring system - Developed a prototype EHR usability scoring system for experimental use as an adjunct to the aforementioned usability tests of five EHRs.

- User interface principles - Wrote 343 EHR-specific user interface design principles.

- User interface design process technical document - Wrote an EHR user interface design process methods, based on vendor best practices, insights gained from the project's research activities, and research team members' judgment and applied design experience.

\subsection{EHRs, EHR users, and EHR tasks}

\subsubsection{Types of EHRs}

There are many different types of EHRs. As a result, EHR user interface design varies widely.

The variability results from many factors, including: 
- User organization type. EHRs may be developed for a variety of clinical organizations, including national or regional healthcare networks, individual hospitals or hospital systems, and private practices of varying size (e.g., large, medium, small, sole practitioner), thereby leading to variation in the applications' functional capabilities.

- Practice setting. EHRs may be designed to support outpatient, inpatient, and/or ambulatory settings.

- Clinical focus. Some EHRs are designed to support particular clinical specialties, such as dentistry, optometry, allied health professions (i.e., physical therapy), oncology, and anesthesiology.

- Functional focus. Whereas some EHRs provide a wide range of functions that are applicable to diverse uses, some EHRs focus on particular functions or features, for example, patient portals.

- Software platform. EHRs may be web-based (i.e., the EHR is accessible using a standard web browser on an internet enabled computer) or client-based (i.e., the EHR is accessible only on the computers on which it is installed). Increasingly, EHR developers are also creating EHRs that are intended for use on mobile devices (i.e., smartphones and tablet computers) in addition to conventional personal computers.

- Development approach. EHRs may be developed as commercial products (i.e., purchased from an EHR vendor), custom-built applications (i.e., developed by an institution's information technology department), or open-source applications based on publically available software code that is widely available for free use.

\subsubsection{EHR users}

EHR users represent a wide variety of professions, clinical specialties, and demographic characteristics, such as the following:

- Providers - hereafter used to refer to clinicians who most commonly prescribe medications, such as physicians, nurse practitioners, and physician assistants

- $\quad$ Nurses - registered nurses (RNs) and licensed practical nurses (LPNs)

- Technicians - including health information technicians (i.e., data analysts) and laboratory technicians

- Pharmacists and supporting staff - pharmacists and pharmacy technicians

- Physicians are further subcategorized as:

o General practitioners - including internists, pediatricians, family doctors, gynecologists, and others who predominantly provide primary care to patients

o Surgeons - including general surgeons, trauma surgeons, neurosurgeons, and cardiothoracic surgeons 
o Medical specialists - including internal medical subspecialists (nephrology, gastroenterology), neurologists, radiologists, and anesthesiologists

o Others - including podiatrists and chiropractors

\section{- Dentists and dental assistants}

\section{- Optometrists and ophthalmologists}

- Clinical specialists in allied health professions - including physical \& occupational therapists, respiratory therapists, speech-language pathologists, dieticians

- Administrative assistants - including office managers, case managers, and administrative assistants

\section{- Billing/coding specialists}

\section{- Medical assistants}

In addition to occupation (or role), EHR users can be profiled according to many criteria, including:

- Demographic traits such as age, gender, ethnicity and other cultural attributes

- Education

- Experience and expertise (particularly pertaining to EHR use)

- Learning style

- Work environment (sometimes called the "use environment")

- Common tasks (as they affect EHR use)

- Performance shaping factors (pertaining to an EHR) such as stress, workload, distraction, sleepiness/fatigue, etc.

EHR developers can develop user profiles (see section 2.1.1.1 - User profiles) to communicate to the entire development team the intended users' demographic characteristics, clinical needs, and common EHR tasks.

\subsubsection{EHR tasks}

EHR developers should perform detailed task analyses to identify potential use errors, some of which might be harmful and warrant risk control measures. Such task analyses are likely to produce a lengthy list of tasks, which may be complemented by task flow diagrams that depict discrete steps. 
EHR tasks may be divided into two broad categories:

- Administrative tasks performed primarily by administrative and support staff, including medical assistants.

- $\quad$ Clinical tasks performed by clinicians such as nurses and providers.

Common administrative tasks, as determined during the course of this research project, include:

- $\quad$ Creating and updating patient records.

- Admitting, discharging, and transferring patients.

- Communicating with patients, staff, and external providers and facilities.

- Printing and faxing documents.

- Managing patient insurance information.

- Scheduling appointments.

- Managing clinicians' schedules.

Common clinical tasks, also as determined during the course of this research project, include:

- Creating patient care plans.

- Writing clinical summaries and notes.

- Entering vital sign measurements.

- Documenting procedures, patient education, and immunization administration.

- Reviewing patient history.

- Reviewing patient clinical information, including vital signs and growth chart.

- Reviewing lab and test results.

- Managing patient problem lists.

- $\quad$ Ordering referrals.

- $\quad$ Ordering tests, labs, procedures, and immunizations.

- Ordering and modifying prescriptions.

- Reconciling patient medication lists. 
- Researching clinical conditions and treatment options.

\subsection{Optimizing the user experience}

\subsubsection{Ensuring use-safety}

The ability to deliver high quality health care is potentially closely tied to the quality of an EHR's user interface design. Simply stated, a poorly designed EHR can induce use errors that could lead to patient injury and even death. A system, if used differently than a manufacturer intended that can lead to hazardous situations. For example, just as an intravenous infusion pump can be misprogrammed to deliver a morphine overdose, a user can enter the wrong medication order into an EHR. Therefore, as EHRs become increasingly integral to healthcare delivery, it will become ever more important to minimize the chance of potentially harmful use errors during their use.

Mirroring the well-established approach to reducing risk that prevails in other industries (e.g., power generation, aviation, transportation, medical devices) where use errors can lead to injury to the user, developers should follow a user interface design process that seeks to identify potentially harmful use errors and decrease their likelihood of occurrence. Fortunately, a usesafety focused design process typically also increases task effectiveness, work efficiency, and user satisfaction.

For example, in the medical device industry, taking steps to reduce the chance of a potentially harmful use error is an important part of a broad-based risk management process. Such efforts make intuitive sense because medical devices can directly cause harm and use errors can lead to infection, laceration, burn, overdose, asphyxiation, and exsanguination. In such cases, risk reduction techniques might include placing automatically activating guards on needles, insulating heat sources, rounding the edges of mechanical parts, shape-coding tubes and associated ports, and adding pressure sensors. The contribution to safety of these design features may be augmented by attention-getting warnings, clearly written and illustrated instructions, and thorough training approaches.

A structured approach to identifying potential use errors using an EHR can lead to conceptually equivalent protections, such as preventing orders of the wrong medication for a given patient by presenting prescription information in a coherent structure, including bold screen titles, using color to effectively highlight important information, and including helpful, non-distracting prompts. The user interface principles presented in section 3 - EHR User Interface Design Principles promote the implementation of these features and many more features to reduce use-related risks associated with the use of EHRs.

\subsubsection{Facilitating task effectiveness}

The first priority is to ensure that user interactions with an EHR are safe, and the second is to ensure that they are effective.

An effective EHR not just enables, but also facilitates task performance. It helps various types of users to complete tasks easily and efficiently. As discussed in section 2.2.4-Reduce use-related 
risks, effectiveness may be a natural by-product of design features principally intended to ensure use-safety. Moreover, an effective EHR is likely to garner compliments from users about its usability.

Usability testing is a productive means to evaluate an EHR's effectiveness. In a usability test, a representative sample of an EHR's intended users attempts to perform selected tasks. Such tasks may be derived from meaningful use criteria. In parallel, they also can be tasks judged to be safety-critical, urgent, frequent, and challenging (see section 2.5.5.2 - Specifying usability test tasks for additional information on task selection).

If a test participant completes a task, but also commits a potentially harmful use error, the task should be characterized a failure. The outcome should be the same - declaration of task failure if there is any need for the test administrator to help the participant complete a task, because such assistance might not be available during actual use and a use error could result.

Developers should aspire to produce EHRs with which all intended users can interact effectively, albeit while recognizing the near impossibility of achieving this goal. Inevitably, certain people at certain times will fail to complete a given EHR task, perhaps due to use errors that cannot be attributed to a design-related root cause. However, if a usability test reveals that several individuals (e.g., 3 out of 30) cannot complete selected tasks, this is a strong indication that the user interface needs modification.

\subsubsection{Optimizing usability}

While use-safety and effectiveness are key components of an optimal user experience, another key component is usability, especially as it relates to user satisfaction. Satisfaction with a technology or software application influences users' willingness to use the product and, when they do, to tolerate aspects of the user interface that do not fully meet their needs. User satisfaction is usually influenced by multiple, interrelated user interface design features, rather than just one or a few features. For example, a user might be satisfied by an EHR that has the following characteristics:

- Intuitiveness enabling the user to perform basic tasks without first reading instructional materials.

- Workflow that closely matches the conventional clinical workflow that existed before EHR implementation.

- Visual appeal resulting from the balanced-looking arrangement of onscreen contents, the use of harmonious colors, easy to interpret icons augmented by text labels, large and three-dimensional looking controls (i.e., buttons), and the use of blank space to separate blocks of information.

Table 1-1 provides additional examples of user interface characteristics that can lead to user satisfaction or dissatisfaction.

Table 1-1: User interface characteristics that can lead to user satisfaction or dissatisfaction. 


\begin{tabular}{|l|l|}
\hline Source of satisfaction & Source of dissatisfaction \\
\hline Harmonious color palette & Garish screen appearance \\
\hline Large, meaningful screen titles & Lack of screen titles \\
\hline Prompt placed in a consistent location & Minimal prompts presented in varying formats \\
\hline $\begin{array}{l}\text { Key information summarized on a single } \\
\text { screen }\end{array}$ & $\begin{array}{l}\text { Key information spread across multiple } \\
\text { screens }\end{array}$ \\
\hline $\begin{array}{l}\text { Confirmation pop-ups associated with } \\
\text { irreversible actions }\end{array}$ & No means to undo an erroneous action \\
\hline $\begin{array}{l}\text { Information, prompts, and alerts provided in a } \\
\text { context-appropriate manner and time }\end{array}$ & $\begin{array}{l}\text { Alerts and prompts that interrupt user } \\
\text { concentration and workflow }\end{array}$ \\
\hline
\end{tabular}

Note that these characteristics could have a positive influence on EHR use-safety and effectiveness. ${ }^{1}$ But, even if they do not, a well-designed EHR should possess these and/or comparable interface characteristics to foster task efficiency and user satisfaction.

The automobile provides a fitting comparator for recognizing the value of usability. Comfortable seats and air conditioning might not be essential for safety and effectiveness, but both are important to users and likely to have a disproportionately strong influence on consumer preference for one car versus another. Similarly, usability is likely to have a disproportionately strong influence on user preference for one EHR versus another.

Accordingly, developers should consider taking the following steps to increase an EHR's usability:

- Address EHR usability concerns along with use-safety and effectiveness concerns during user research activities.

- Write usability-focused user interface requirements.

- Conduct benchmarking studies of competitor EHRs to better understand user expectations, prominent design trends, and opportunities for market differentiation through usability improvements.

- Establish quantitative usability goals that will be upheld with the same diligence as other technical or software engineering goals (e.g., software response time).

- Invest user interface design effort into making an EHR visually appealing and intuitive to use.

- Conduct a variety of usability-related evaluations, such as expert reviews and usability tests.

\footnotetext{
${ }^{1}$ Tractinsky, N., Katz, A. S., \& Ikar, D. (2000). What is beautiful is usable. Interacting with computers, 13(2), 127-145.
} 
- Iterate the design process (i.e., establish user interface requirements $\rightarrow$ design $\rightarrow$ model $\rightarrow$ test) to the extent necessary to ensure EHR usability.

Developers are likely to find that usability is often a natural by-product of efforts to ensure an EHR's use-safety and effectiveness because an investment in user-centered design can have a positive influence on all three factors. However, developers might have to invest at least some extra effort to ensure an EHR's usability, which should pay off well in terms of higher user satisfaction. 


\section{User Centered Design and Evaluation Methods}

This section describes an approach to developing and validating an EHR's user interface so that the application is reasonably safe and effective. The intent is to promote good development practices without being overly prescriptive or stalling innovation.

The need for this guidance is based on the fact that EHR development practices to date have not consistently considered users' needs, particularly in the context of less than optimal conditions of actual clinical work. As a result, some EHRs have been vulnerable to use errors.

Some EHR functions, such as medication prescribing, are potentially, safety-critical similar to the functions on medical devices such as defibrillators, intravenous infusion pumps, and anesthesia delivery machines. Even EHR functions perceived by some to be "low risk," such as clinical documentation capabilities, have been shown to contribute to patient harm. For example, if a provider fails to document or incorrectly documents a patient's medical condition or allergy, the consequences associated with subsequent care (or lack thereof) could be catastrophic.

As such, developers should commit themselves to developing EHRs: taking a user-centered design approach to ensure EHR use-safety and effectiveness, as well as EHR usability and user satisfaction.

Note that a particular EHR user interface development effort is likely to involve parallel activities and iterative cycles that are difficult to describe in a linear narrative. Therefore, developers should consider opportunities for sequencing the steps described below in a manner that complements other EHR development activities and proceeds in a productive and efficient manner.

Insights shared in this section are based on the following:

- $\quad$ Findings from research into the best practices already employed by selected EHR developers, including those that have developed a wide array of EHR applications to be sold to and implemented within diverse clinical settings, including physician's offices, outpatient clinics, and hospitals ${ }^{2}$.

- Review of human factors engineering and related literature, including many guidance documents and standards, on best user interface design practices.

- Research team member experience conducting user research, designing user interfaces, and evaluating user interfaces for a multitude of products including software applications,

\footnotetext{
${ }^{2}$ The authors express their gratitude to the National Center for Human Factors in Healthcare, Medstar Health, Washington DC., which shared the results of their own research into best user interface design practices among EHR developers; work they performed under contract to the Office of the National Coordinator in 2013. Some of their findings are also documented in the following article: Ratwani, R. M., Fairbanks, R. J., Hettinger, A. Z., \& Benda, N. C. (2015). Electronic health record usability: analysis of the user-centered design processes of eleven electronic health record vendors. Journal of the American Medical Informatics Association, ocv050.
} 
websites, consumer products, industrial products, military equipment, power generation equipment, aviation equipment, and medical devices.

Figure 2-1: An illustration of a sample implementation of a user-centered design process.

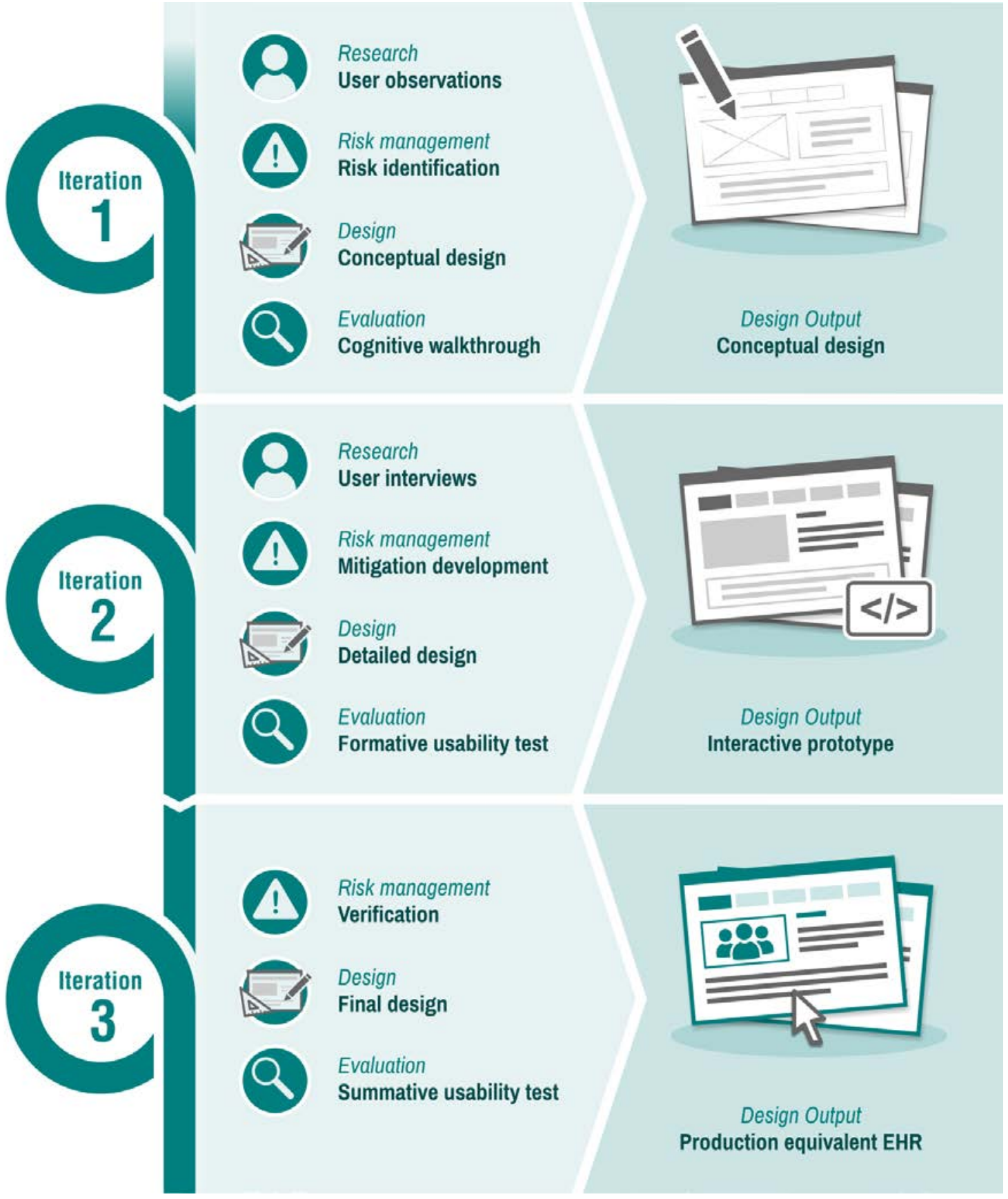

The balance of this section covers the following major elements of a user-centered approach to designing an EHR's user interface:

- Conducting user research. 
- Managing use-related risk.

- Designing the user interface.

- Evaluating the user interface.

\subsection{Conducting user research}

The primary goal of user-centered design is to develop user interface design solutions that meet users' needs, a key one of which is to operate the user interface safely and effectively. This goal can be accomplished in part by taking energetic steps to identify user needs. Even though an EHR developer might already have (or believe it has) extensive knowledge of user needs based on the developer's prior market research activities or general knowledge, some specific user research is usually still warranted.

It is important to emphasize that clinicians who are employed by a developer, or are hired as consultants because of their special expertise, are never representative of all of the EHR's intended users. Thus, active research involving a cross-section of typical users is beneficial.

This subsection describes how developers can identify user needs, convert the needs into user interface requirements, and then use the requirements to drive the user interface design effort.

\subsubsection{Determine user needs}

As suggested above, a broad and deep understanding of an EHR's users' needs is fundamental to designing an EHR user interface that is safe, effective and easy to use. Common and effective methods for developing this understanding involve (1) observing the intended users at work, and (2) speaking with them about their EHR-related needs and desires.

Observation and interview methods vary in name and emphasis depending on what type of professionals perform the research. The possibilities include people trained in the overlapping fields of human factors, ethnography, interaction design, information design, marketing, communications, and related disciplines. For example, human factors specialists use the term contextual inquiry when referring to interviewing people while they work. Ethnographers speak of performing ethnographic research as a specialized means of observing people unobtrusively to gain a more accurate understanding of how they work and performance-shaping factors related to the environment and system in which they work. Market researchers speak about conducting interviews and focus groups to capture the voice of the customer.

During such research activities, users might articulate their needs such as those presented below in hypothetical, first-person forms:

- "I want the patient's chart to clearly show their name, age, hospital ID number, principal medical condition, and maybe even a photograph for ease of recognition."

- "I don't like having to go to many screens to get the information I need to make a clinical decision. The key information should be on one screen. Stuff it in if you need to." 
- "I need the system to check for drug interactions and grab my attention if there is a problem."

The overarching point is that observing and speaking with users can generate useful insights regarding the qualities that will make an EHR safe, effective, usable, and satisfying. As such, best practices suggest that one or more development team members should conduct some form of user research with a heavy focus on EHR safety and effectiveness.

A critical element of any user research method is to get beyond users' current practices (i.e., the tasks they currently perform with the tools they currently use) to identify their underlying work goals and priorities. For example, the best software products do not simply convert a paperbased form into an electronic one. Rather, by first gaining a thorough understanding of the underlying associated work, developers can produce electronic forms that harness the value added by a computer to improve task safety and effectiveness, as well as usability (encapsulating efficiency, intuitiveness, and satisfaction).

Further, neither interviews nor observations alone will suffice. Observations, no matter how extensive and detailed, in the absence of user interviews, will not reveal users' underlying reasons or motivations for their actions and thus miss essential aspects of users' real needs. On the other hand, relying solely on users' descriptions of their current practices and their preferences for future EHRs will inevitably yield suboptimal products. Therefore, it is important to observe and speak to users.

Ultimately, EHR developers should adopt a user research approach of its own choosing while considering the following advice:

- Extend the research to cover the widest possible range of intended EHR users, including primary and secondary users.

- Extend the research to cover not only common use scenarios, but also uncommon ones (i.e., edge cases), especially if those use scenarios are safety-related.

Common deliverables for documenting user research include a prioritized list of user needs, a list of use scenarios (i.e., common and high risk tasks), and a description of user characteristics, sometimes expressed as user profiles and/or user personas, which are described below. Research findings may be described in stand-alone documents of the research activities or converted directly into user interface requirements (see section 2.1.6 - Develop user interface requirements). 


\subsubsection{User profiles}

A user profile is a description of users who are members of a distinct user group (i.e., a sub-group of the overall intended user population). User profiles may be brief (e.g., $1 / 2$ page) or expanded (23 pages) documents. They typically cover characteristics that could have bearing on an EHR's user interface design, such as the following:

- Demographic traits such as age, gender, ethnicity and other cultural attributes

- Education, experience and expertise (particularly pertaining to EHR use)

- $\quad$ Learning style

- Work environment

- Common tasks (as they affect EHR use)

- Performance shaping factors (pertaining to an EHR)

Performance shaping factors might include the following details ${ }^{3}$ :

- General health and mental state (stressed, relaxed, rested, tired, affected by medication or disease) when using the EHR

- Sensory capabilities (vision, hearing, touch)

- Coordination (manual dexterity) as it might influence onscreen navigation and data entry

- Cognitive ability and memory

- Knowledge about EHR operation and the associated functions (e.g., physician order entry)

- Previous experience with EHRs (particularly EHRs that serve similar purposes)

- Previous experience using software applications (including desktop, web-based, and mobile applications)

- Expectations about how an EHR will operate,

- Motivation

- Ability to adapt to adverse circumstances associated with EHR use (e.g., using it in an emergency use scenario)

\footnotetext{
${ }^{3}$ FDA: Medical Device Use-Safety: Incorporating Human Factors Engineering into Risk Management, 3.2.2 Medical Device Users, Page 12, Document issued on July 18, 2000.
} 
- Amount of cooperation with other EHR users (e.g., individual EHR use versus teambased use)

- Work pace (e.g., slow and deliberate or fast and decisive)

Appendix D: User Profiles of 10 EHR Users support the development of user interface requirements that can be linked to the traits and work habits of specific user groups.

\subsubsection{Personas}

A persona is an alternative or a complement to a user profile. Typically a 1-2 page narrative, it describes a particular but usually hypothetical (or at least an actual but de-identified) individual rather than members of an overall user population. Developers may create multiple personas for each user group, with each persona reflecting characteristics and behavioral traits amalgamated from user research findings.

Personas are often used by market researchers to describe specific types of customers. They often include potentially relevant personal life details (e.g., hobbies) and behavioral descriptions that are not normally included in a user profile. Some user interface developers prefer personas over user profiles because they believe that it can be more productive to design an application to meet the needs of selected individuals rather than the general needs associated with distinct types of users. They may find it easier to imagine and empathize with hypothetical individuals rather than more general user profiles. While the comparative values of both end products are debatable, either approach can support the development of user interface requirements.

\subsubsection{Identify work environment characteristics}

A solid understanding of the environments in which people will interact with an EHR (the "use environments") also provides a foundation for making good user interface design decisions.

It is practical to study EHR users' work environments at the same time one learns about user's needs and preferences (i.e., while conducting user interviews or observations - see section 2.1.1 - Determine user needs). Insights are typically documented in the form of use environment descriptions; one for each significant type of use environment.

Similar to a user profile, a use environment description may be a brief (e.g., $1 / 2$ page) or expanded (e.g., 1-3 page) description of a particular setting. Use environment descriptions discuss characteristics that might affect an EHR's user interface design, such as:

- EHR placement within the use environment and expected viewing distances

- Availability of work surfaces

- Network connectivity

- Connected and adjacent medical devices

- Seating (affecting viewing angle) 
- Noise levels, lighting, and other environmental factors

- Sources of distraction (e.g., phone calls, patient requests)

- Concurrent tasks and activities

- Nearby personnel

- Potential for unauthorized use (e.g., by hospital visitors)

Note that an EHR might be used on a fixed workstation, such as those installed in central nursing stations, patient rooms, and offices; on a rolling or hallway workstation; or on mobile devices (e.g., tablets, smartphones) that travel throughout a clinical environment.

Similar to user profiles, use environment descriptions support the development of user interface requirements pertaining to such factors as visual and physical access to the EHR display and pointing device (if not a touchscreen), and conditions and events that could interrupt interactions with the EHR.

\subsubsection{Define preferred workflows}

Whether or not people are accustomed to healthcare delivery workflows involving existing EHRs, they will be accustomed to particular workflows. Those workflows can vary widely in terms of safety, effectiveness, efficiency, and the degree to which workers consider them to be satisfactory. Developers should study existing workflows to determine which characteristics must or should be preserved in a new EHR, and most importantly, how the EHR could enhance or facilitate task safety, effectiveness, and efficiency.

There are ample examples of EHR's technical limitations or user interface design flaws that have led to undesirable changes in clinicians' workflow. Developers should be acutely aware that changes to existing workflows might be disruptive and even unsafe, sometimes in unanticipated ways. As such, they should analyze the potential effects of all changes in workflow and proceed with solutions that change workflow only when there is a significant benefit to the EHR users.

\subsubsection{Determine and evaluate common workflows}

Developers should study existing workflows before designing an EHR, noting that an EHR will invariably affect future workflows to some extent. Specifically, a developer should determine how clinicians complete EHR-related tasks in the absence of an EHR (i.e., using paper-based records) and/or with a legacy EHR system.

It is common to document existing and anticipated workflows in the form of flow charts that depict user actions and decisions in response to computer actions.

\subsubsection{Define use scenarios}

In the context of EHR development, a use scenario is a description of possible interactions between the user and the application. Use scenario details may include: 
- Types of users involved in the use scenario

- Use environment in which the use scenario occurs

- Conditions influencing user interactions (e.g., workload, task urgency, experience using other EHRs)

- Tasks performed during the use scenario

Common use scenarios and their associated details can be identified by speaking with EHR users about how they use their current EHRs or how they currently perform tasks that will eventually performed using an EHR under development. Direct observations are useful in both identifying potential use scenarios and in confirming scenarios derived from user interviews.

Developers should identify a wide range of EHR use scenarios as an input to the user interface requirements development and risk management process.

The following are summaries of a few sample use scenarios:

- Neonatologist using an EHR in a neonatal intensive care unit to order an emergency medication for a newborn baby.

- Medical assistant using an EHR in an outpatient clinic to schedule a patient's follow-up visit.

- During shift-turnover, a registered nurse starting her shift by using an EHR in a hospital's medical/surgical unit to review current medication orders for the 8 patients for whom she will be caring.

\subsubsection{Develop user interface requirements}

Just as technical requirements can drive the design of an EHR's database structure or software architecture, user interface requirements can drive user interface design. Requirements can be derived from the following sources:

- User interface design principles, such as those found in this document and many other articles, websites, textbooks, and standards

- Needs expressed by prospective EHR users, documented in user research documents, user profiles, or personas

- Use environment characteristics that influence users interactions with the EHR, documented in use environment descriptions

- User interface designer judgment, informed by education, experience, and design talent

- Usability problems and use errors identified in legacy or competitor EHRs, in the literature, or in publically available databases 
- User interface design principles applicable to the particular operating system on which the EHR runs

There should be sufficient user interface requirements to address important user needs, ensure adequate EHR functionality, prescribe error resistant and error tolerant features, and imbue the final EHR with other relevant usability-related characteristics. As such, it is common for EHRs to have many user interface requirements, typically numbering in the dozens and perhaps exceeding one hundred.

\subsubsection{Write usability goals}

Recognizing that they are not normally a mechanism for ensuring an EHR's use-safety, developers may still write usability goals that quantitatively specify the desired quality of user interaction with and the desired user impressions of the EHR. Usability goals can be established based on usability evaluations of legacy and competing EHRs, competitive market goals, or the developer's aspirations for the overall quality of the EHR's user experience. Data collected during usability testing efforts can then be compared to the established usability goals, enabling the developer to determine whether the evolving or final EHR design meets the target metrics.

Usability goals gained favor in the 1990s and continue to be embraced by some developers as a means to elevate an application's usability to the same level as other, testable quality measures. Meanwhile, others believe the technique can mislead developers to accept a sub-optimal level of application dysfunction. For example, if a developer sets the apparently high usability goal that $90 \%$ of new users will be able to complete a task, meeting the goal would still allow that $10 \%$ of new users may unable to complete that task during actual use. On the other hand, setting a usability goal of $99 \%$ is simply not feasible since hundreds of users would need to be tested to assure that the goal has been attained.

Therefore, best practice suggests limiting the use of usability goals to matters of general usability, efficiency, intuitiveness, and appeal, but to eschew them when it comes to matters of safety. Clearly, percentage-based usability goals are inappropriate for safety-related task performance because even a single instance of failure is cause for serious investigation and design revision. In contrast, time based usability goals might be valuable and provide a straightforward basis for performance assessment.

In clear cases where the time to perform a task is safety critical (e.g., ordering a stat ECG), ensuring that an EHR meets a time-based usability goals could be an important step toward validating an EHR's safe use.

Typically, a complete set of usability goals includes some that are objective and thus observable and others that are subjective and thus require users to express their opinions. Objective usability goals are often based on the time taken to perform a selected task or the rate at which new users are able to complete a selected task successfully. Subjective usability goals call upon individuals who have used an application to express their opinion about it, typically using a rating scale. Table 2-1 presents a sample of objective and subjective goals pertaining only to an EHR's usability. 
Table 2-1: Sample objective and subjective usability goals.

\begin{tabular}{|l|l|}
\hline Objective usability goal & \multicolumn{1}{l}{ Subjective usability goals } \\
\hline $\begin{array}{l}\text { 85\% of new users shall successfully log into } \\
\text { the EHR on their first attempt. }\end{array}$ & $\begin{array}{l}\text { On average, new users shall rate the EHR's } \\
\text { ease-of-use as } 4.5 \text { or better (scale: } 1 \text { = poor, } \\
7=\text { excellent) }\end{array}$ \\
\hline $\begin{array}{l}90 \% \text { of new users shall be able to load a } \\
\text { patient's chart within } 30 \text { seconds or less. }\end{array}$ & $\begin{array}{l}\text { On average, new users shall rate the patient } \\
\text { chart's readability as } 5 \text { or better (scale: } 1= \\
\text { poor, } 7=\text { excellent) }\end{array}$ \\
\hline $\begin{array}{l}75 \% \text { of new users shall be able to enter a } \\
\text { patient's vital signs in } 5 \text { minutes or less. }\end{array}$ & $\begin{array}{l}\text { On average, new users shall rate the EHR's } \\
\text { intuitiveness as } 3.5 \text { or better (scale: } 1=\text { poor, } \\
7=\text { excellent) }\end{array}$ \\
\hline
\end{tabular}

Developers have the prerogative to make the success criteria for each goal (e.g., target task time for an objective goal, target average rating for a subjective goal) based on their ambitions for the EHR's interaction quality. Ideally, the set criteria should be based on user research or usability testing results (e.g., average task times collected from a usability test of a legacy or competitor's EHR).

\subsection{Managing use-related risk}

Another goal of user-centered design is to develop user interface solutions that are less vulnerable to potentially harmful use errors. This goal can be accomplished by taking the necessary steps to understand how EHR users might err and either preventing such occurrences altogether or mitigating their negative effect. This process is called use-related risk management.

In summary, use-related risk management involves identifying hazards and associated use errors, estimating the risk associated with the use errors, and reducing the risk of use errors that could cause significant harm. The requisite risk management steps are described below.

\subsubsection{Identify hazards and associated use errors (top-down approach)}

One might normally think about hazards as physical things that can cause harm. In clinical environments, for example, use of medical devices can introduce hazards such as an unguarded needle, energized electrode, leaking blood tubing, or unshielded radiation beam. Each of these hazards could cause harm to a patient or a clinician. In this case, to address these risks of harm, device developers are compelled to identify hazards associated with the use of their devices and to implement protections against use errors that could expose users to hazards (i.e., create hazardous situations) and lead to harm. The implementation of risk control (i.e., reduction) measures is often termed risk mitigation. The "top down" approach described here (starting with identification of possible hazards and the harms they can cause, and then analyzing how use of the product could lead to such harms, is typically called a use-related hazard analysis.

For example, in contrast to therapeutic medical devices such as infusion pumps, a use error committed with an EHR cannot harm a patient directly. However, EHR users can commit use 
errors that create hazardous situations whereby the actions or inactions resulting from EHR use can be injurious or even deadly. Therefore, it is incumbent on EHR developers to identify potential hazards, just as would the developer of nuclear radiation delivery software, dialysis machine, or anesthesia workstation.

Table 2-2 presents sample use errors and possible harms.

Table 2-2: Sample possible harms and use errors, which may be derived from a fault tree or similar analysis.

\section{Possible harms \\ Intended patient does not receive treatment, resulting in a range of possible harms ranging from suboptimal disease management to death \\ Incorrect patient receives unnecessary procedure, resulting in a range of possible harms ranging from temporary and/or mild discomfort to permanent injury or death \\ Delay in diagnosis or suboptimal disease management due to additional time required to recognize wrong test order and re-order correct test \\ False positive diagnosis based on test results of wrong test, resulting in unnecessary treatment}

Patient is subsequently (re)prescribed that medication, resulting in a deadly allergic reaction

Delay in follow-up care for intended patient, resulting in suboptimal disease management.

Patient receives unnecessary medication, resulting in anything from mild discomfort to deadly allergic reaction

Patient does not receive influenza vaccination and contracts a form of influenza, resulting in a life-threatening pulmonary infection

Patient does not receive the diagnostic test, resulting in delayed detection of a critical diagnosis (e.g., breast cancer) thereby appreciably worsening prognosis
Use errors

Ordered procedure for the wrong patient

\section{Ordered wrong test}

Stopped a medication to which a patient had an allergic reaction but did not add medication to the patient's allergy list

Scheduled appointment for wrong patient

Ordered an unnecessary medication

Documented that an influenza vaccine had been administered without actually ordering (or administering) the vaccine

Overlooked clinical reminder and failed to order a diagnostic screening test (e.g., a mammogram) 
Risk analysts should consider how user interactions with the EHR could expose people to the following types of hazards:

- Biological / clinical (including both over- and under-diagnosis or treatment)

- Chemical (including medications)

- Electrical

- Mechanical (i.e., physical injury)

- Psychological / emotional

- Radioactive

Once hazards are identified, developers should determine all of the possible use errors that could lead to exposure of the patient (or even provider or bystanders) to one or more hazardous situations. Fault tree analysis is a common way to determine use errors that could expose the user to a hazard and potential harm. The technique calls for analysts to start with a harm and then work backwards to determine what event or series of events, including one or more use errors, can lead to the harm.

\subsubsection{Identify potential use errors and associated hazardous scenarios (bottom-up approach)}

In addition to taking the top-down approach of identifying hazards and linking them to use errors that can lead to exposure, developers can take a bottom-up approach that calls for identifying potential use errors and then determining what harm the errors could cause. This process is often termed a use-related failure modes and effects analysis.

The first step in this approach is to perform a task analysis, defining each step users will take to complete particular tasks with the software. This poses a significant challenge when an EHR's user interface design is incomplete or perhaps not substantially advanced beyond an initial concept. In such cases, the analysis will be more speculative, but the results can actually guide the ensuing design effort. When there is already a preliminary user interface design, or a new version will be heavily based on a previous version (i.e., share many of the predecessor's characteristics), the task of tracing the interactive steps involved in the selected tasks is relatively straightforward.

A user-EHR interaction can be deconstructed into one or more of the following elements:

- Perception - acquisition of information (e.g., reading a growth chart)

- Cognition - mental processing of the information (e.g., deciding if a child is growing at a healthy pace and what to do if this is not the case)

- Action - taking action (e.g., ordering a diagnostic test or a medication) 
Deconstructing tasks into these categories constitutes a PCA analysis (the acronym PCA stands for Perception-Cognition-Action). EHR developers are encouraged to perform a PCA analysis to identify potential use errors, noting that one can generate a large set of potential use errors by exploring the range of perceptual errors (e.g., not noticing an on-screen alert), cognitive errors (e.g., incorrectly recalling a vital sign value), and action errors (e.g., clicking on the wrong patient name).

A PCA-type analysis is likely to generate hundreds of potential use errors. While many such errors are unlikely to be hazardous, some might lead to harm. A risk analysis should be performed to identify those posing significant risk.

\subsubsection{Determine risks associated with use errors}

The dominant form of use-related risk analysis calls for developers to use numerical rating scales to characterize both the probability (i.e., likelihood) of a use error, and the severity of the harm that can arise due to that use error. The resulting risk estimation for each use error is derived from the product of the probability and severity ratings.

Commonly used rating scales and a risk estimation matrix are shown in Table 2-3 and Figure 2-2. Using these ratings scales, a developer might estimate the probability of a particular use error to be a 3 (Occasional) and the severity of the harm it might cause as a 4 (Critical), resulting in a risk of $12(3 \times 4)$. According to the sample risk estimation matrix, the use error falls in the highest part of the yellow zone, suggesting the need to decrease the risk through user interface redesign or other risk mitigations. 
Table 2-3: Levels of probability and severity associated with use errors and their potential harms (adapted from ISO 14971:2007 Medical devices -- Application of risk management to medical devices).

\begin{tabular}{|l|l|}
\hline Severity & $\begin{array}{l}\text { Probability (i.e., Likelihood) } \\
\begin{array}{l}5-\text { Catastrophic } \\
\text { Results in patient death. }\end{array}\end{array}$ \\
\hline $\begin{array}{l}\text { Likelihood of occurrence is greater than or } \\
\text { equal to } 10^{-3} .\end{array}$ \\
$\begin{array}{l}\text { Results in permanent impairment or life- } \\
\text { threatening injury. }\end{array}$ & $\begin{array}{l}4-\text { Probable } \\
\text { Likelihood of occurrence is less than } 10^{-2} \text { and } \\
\text { more than } 10^{-4} .\end{array}$ \\
\hline $\begin{array}{l}\text { Results in injury or impairment requiring } \\
\text { professional medical intervention. }\end{array}$ & $\begin{array}{l}3-\text { Occasional } \\
\text { Likelihood of occurrence is less than } 10^{-4} \text { and } \\
\text { greater than } 10^{-5} .\end{array}$ \\
\hline $\begin{array}{l}\text { Results in temporary injury or impairment not } \\
\text { requiring professional medical intervention. }\end{array}$ & $\begin{array}{l}\text { Likelihood of occurrence is less than } 10^{-5} \text { and } \\
\text { greater than } 10^{-6} .\end{array}$ \\
\hline $\begin{array}{l}1-\text { Negligible } \\
\text { Inconvenience or temporary discomfort. }\end{array}$ & $\begin{array}{l}1-\text { Improbable } \\
\text { Likelihood of occurrence is less than } 10^{-6} .\end{array}$ \\
\hline
\end{tabular}


Figure 2-2: Use error probability and severity matrix.

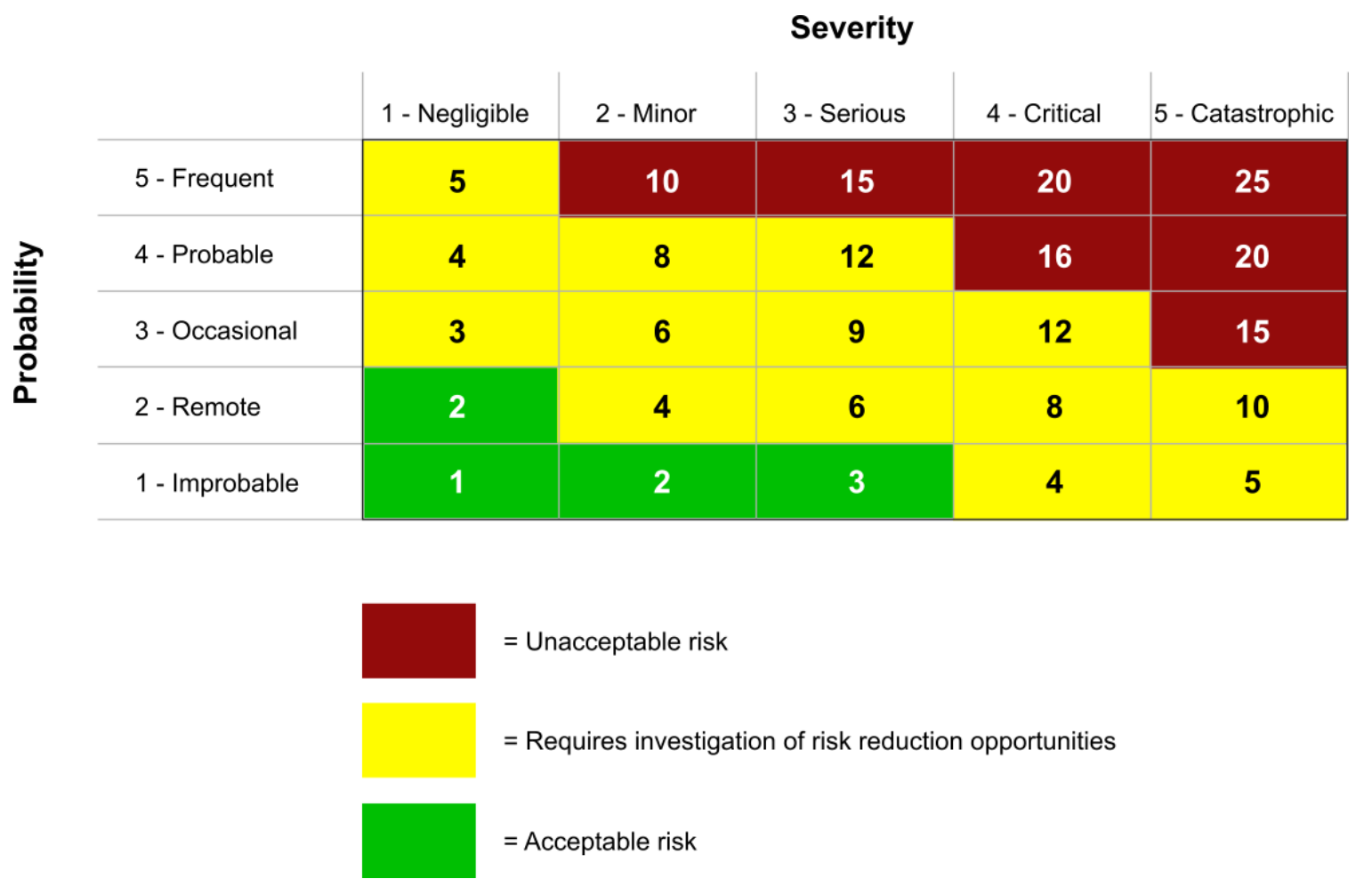

Depending on a developer's risk management philosophy, it may explore ways to reduce all risks that start in the red and yellow zone, ideally dropping them into the green zone or lower region of the yellow zone. For example, in the medical device industry, manufacturers normally view risks in the red zone as unacceptable and mandating risk reduction, while risks in the yellow zone as warranting an exploration of opportunities to reduce risk through various strategies.

For example, medical device developers refer to ISO 14971: Medical devices -- Application of risk management to medical devices, for conceptual and detailed guidance on how to approach a risk analysis properly. While there is an ongoing effort to develop a national risk management standard for EHR development, in the interim, EHR developers should refer to ISO 14971 as well as existing national and international standards software risk management.

In practice, it is quite difficult to accurately estimate the probability of a use error occurring. For example, there is little basis for estimating the chance that a user will not detect an onscreen alert. Developers can review field experience with predecessor products to help estimate probabilities, but reliable use error reporting is uncommon, which undermines the integrity of the analysis. Therefore, probability estimates usually are educated guesses that could be wrong by an order of magnitude. As a result, the emerging trend in high-risk industries (for example, including medical device industry) is to focus more on severity of harm when deciding if there is a need for risk reduction measures. For example, using the rating scale presented above, a conservative approach would be to explore ways to eliminate the risk of any use error that has a severity rating of 3 (Serious) or above. 
Other factors that play a role in risk management are the detectability of a use error and the ability of the user to correct the error if he or she detects it. Thus, developers may choose to factor these variables into their risk analyses. In such a scheme, for example, low use error detectability would increase risk, and high use error detectability would reduce risk. These factors also present opportunities for risk mitigation. For example, a user interface could be redesigned to make a use error more apparent and, therefore, more recoverable due to its timely detection.

\subsubsection{Reduce use-related risks}

When the likelihood of a user committing a use error is high and the resulting harm can be severe, the risk must be reduced to a level that the developer's risk management process stipulates is an acceptable level. EHR developers have little, if any, control over the severity of the harm that can result from an EHR use error, such as overdosing a patient, because the harm is a matter of biology rather than technology.

Therefore, EHR risk control measures focus on reducing or eliminating the chance of a use error occurring, or providing means to detect and recover from the error before it causes harm.

Applying the user interface design principles presented in EHR User Interface Design Principles is one way to reduce the risk of use error occurrence.

Table 2-4 lists several sample principles and their intended use-safety benefit.

Table 2-4: Sample principles and their intended use-safety benefit.

\section{Guideline \\ Intended use-safety benefit}

Persistently display and highlight important patient information, such as disease mechanisms, current medications, and allergies, on patient information displays. Information may be presented in a persistent header, "dashboard," "whiteboard" or similar user interface element that contains the key information. Do not permit the patient name to become hidden due to paging or scrolling, for example.

Present users with options that are operational mode-appropriate. For example, when prescribing medication to a pediatric patient, present medication dose options that are appropriate to children rather than adults.

Reduce the chance that a user will place a medication order for the wrong patient
Reduce the chance that a clinician will enter a pediatric patient's weight using the wrong units of measure 
Guideline

Provide users with a direct means to perform emergency tasks, rather than requiring them to first perform non-essential tasks. Relatively low importance and non-urgent steps could cause an undesirable delay that could lead to patient harm. For example, enable appropriate users to order an emergency medication (e.g., tissue plasminogen activator (t-PA) for ischemic stroke) without having to complete a patient admission form ahead of time.
Intended use-safety benefit

Reduce the chance that there will be a delay in delivering therapy to a new EHR patient with an acute stroke who has not yet been "admitted" in the system

The principles provided in section 3.2 - Principles can serve as a good general starting point for identifying mitigations. However, certain risks will likely require developers to develop specific, custom mitigations based on the developed EHRs distinct design.

To produce a reasonably safe EHR, developers must be prepared to modify an EHR's user interface in response to evidence and expert judgments that certain user interface aspects are leading to potentially harmful use errors. Human factors experts describe a hierarchy of risk mitigations where the most effective (and often the most effortful) is to redesign the user interface to eliminate the potential of use error. Often, effective but somewhat less reliable risk mitigation strategies include making the correct actions more obvious by providing cues (i.e., affordances), making the error less likely through preventative measures (i.e., constraints), or making the use error or its consequences more detectable. Generally, warnings, alerts, and instructions that essentially inform the user to "be careful" or "avoid making this error" are much less effective mitigations.

For example, experience in the medical device industry suggests that, in the interest of minimizing disruption to a product development effort, some developers eschew significant changes by defining a user interface designs as "frozen" (i.e., not subject to change) ahead of a final user test of the device. Instead, they favor changes to user documentation, product labeling, or user training in response to suboptimal test results. However, modifications to documentation and training are generally considered weak risk mitigations as compared to user interface design modifications. For example, it is more effective to require users to confirm an important parameter setting, rather than place an advisory in the user manual for users to double-check settings.

As discussed in section 2.4 - Evaluating the user interface, developers can conduct usability tests to evaluate the effectiveness of chosen risk reduction measures. If the risk mitigations are effective, users will be able to complete tasks without committing safety-related use errors. If even one user still makes a safety-relevant use error despite the mitigation(s), then different or additional risk control measures are warranted. 


\subsection{Designing the user interface}

A comprehensive description of an EHR user interface design process is outside the scope of this document. Otherwise, this document would devolve into a lengthy discussion of such topics as:

- Exploring a range of conceptual models that best satisfy the EHR's function requirement and user interface requirements, and foster the formation of simple and accurate mental models among users.

- Considering a range of user interface structures that reinforce a simple and accurate mental model, provide rapid access to frequently used as well as important functions, and afford a logical and desirable workflow.

- Developing multiple visual design solutions that emphasize the right information and controls while also addressing aesthetic goals.

- Converging on a user interface design solution that combines the strongest conceptual model, user interface structure and visual design solutions.

Important steps:

1) Ensure that user interface requirements drive the design, rather than letting technical software requirements and programmers' personal judgments override the user needs expressed in the form of user interface requirements.

2) Repeatedly evaluate the design-in-progress to determine if there are any new opportunities for use error that should be a new input to the risk management process. For example, it may be quite productive to evaluate a design near the completion of the following stages of development:

- Conceptual design - which might be represented in the form of several sample screens (e.g., wireframes) and a user interface structure diagram.

- Preliminary design - which might be represented by a medium-fidelity, interactive prototype of selected portions of the application.

- Detailed design - which might be represented by a high-fidelity, interactive prototype of most portions of the application.

- Final (production-equivalent) design - which should be represented by a fully functional (i.e., production-equivalent, ready for release) version of the EHR, including the supporting learning aids.

\subsubsection{Fulfill user interface design requirements}

As suggested above, the user interface design effort should be driven by the user interface requirements that, in turn, are based on user needs and preferences as well as risk management efforts. In practice, this means that user interface designers should be held accountable for meeting the user interface requirements as opposed to designing the user interface according to 
team members' strictly personal judgments and/or programming expedience. Approached properly, meeting user interface requirements should not be an obstacle to innovation. Rather, taking a disciplined approach to user interface design might actually reveal innovation opportunities.

\subsubsection{Re-analyze tasks and perform additional risk management}

As an EHR's user interface evolves and becomes more complete, it enables a more detailed tasks analysis. Instead of speculating about how users will perform tasks as a basis for identifying potential use errors, risk analysts can perform increasingly specific and accurate task analyses. This results in a more accurate and comprehensive set of potential use errors, although the results should substantially overlap with results of the initial analysis. Updated task analysis results - specifically the updated list of potential use errors - should be an input to a continuing risk management effort.

\subsection{Evaluating the user interface}

\subsubsection{Evaluate initial concept(s)}

There are several ways to evaluate a user interface design in the early stage of its development. A couple common approaches are detailed below.

\subsubsection{Conduct expert review}

An expert review is a productive way to identify an EHR's user interface design strengths and opportunities for improvement at various stages of its development. For example, experts can evaluate an EHR when it is at the concept development stage and expressed in the form of sample screens comprising one or just a few important workflows. They can evaluate an early interactive prototype that includes only certain functions. And, they can evaluate more complete prototypes as well as finished products, the latter for the purpose of identifying opportunities to produce an improved, next-generation version of an EHR already in use. Additionally, developers can engage expert reviewers in an extended dialogue regarding a particular finding or comment, whereas it might be difficult or impractical to obtain additional feedback from usability test participants who have limited time to spend in an EHR evaluation. Therefore, it might be advantageous for an EHR developer to initiate two, three, or even more expert reviews over the course of a development effort, noting that such reviews can be completed quickly and at relatively low cost.

An expert review can be a valuable complement to formative usability testing (see section 2.4.1.1 - Conduct expert review) because it can generate different types of findings over a wider range of time that might arise from an analysis of test data. However, developers should not consider expert reviews to be a complete substitute for formative usability testing because certain design strengths and opportunities for improvement become evident only when actual users perform representative tasks with an EHR.

An expert review calls for one or more experts to inspect an EHR and state opinions about its interactive qualities, including the good and bad. Participating experts need not pass a qualifying 
test of their knowledge and judgment in the same manner as a pilot who must be certified to fly a particular airliner. Rather, the standard of care for selecting experts is up to the developer; it is a judgment call.

Still, certification as a human factors professional holding a degree in a user interface designrelated field and similar credentials are certainly hallmarks of a genuine expert. A degree of objectivity is advantageous as well, which is why it makes little sense for members of the development team to conduct their own expert reviews. For this reason, developers often turn to outside consultants, or at least co-workers who are not members of the particular EHR development team, to conduct such reviews.

Ultimately, the productivity of a particular review depends on the selected expert(s) to possess a suitable base of knowledge and experience in such areas as, but not limited to, the following:

- User interface design and its variants (e.g., user experience design, interaction design, user interface architecture)

- Human factors engineering (aka usability engineering)

- Human-computer interaction

- Visual design

- Clinical domain knowledge (i.e., clinical terms, tasks, and overall workflows)

An expert review is a flexible process, one that is usually shaped by a reviewer's or review team members' preferred practices, which may include the following activities:

- Develop a first impression by exploring the EHR and attempting to perform tasks before getting a complete introduction to the EHR through briefings, training, and readings.

- Learn about the EHR's intended uses, users, and the environments in which the EHR will be used.

- Review user interface design goals with the development team.

- Participate in training, most likely of a condensed form but possibly in its fullest form, to use the EHR. Alternatively, observe all or a portion of an actual training session.

- Scan or read in full the EHR learning aids.

- Establish a set of general (i.e., global) and specific (i.e., local) user interface design topics upon which the review will focus. The set of topics could mirror the ones that guided the expert review that the research team performed on five EHRs (see section 4.4.1 - Expert review categories), or an alternative set that may be assembled based on the reviewers' judgment. 
- Document user interface design strengths and opportunities for improvement (i.e., weaknesses, shortcomings). The term "opportunities for improvement" is preferable because it is a more constructive expression that is aligned with the end-goal of producing an optimal EHR user interface.

Optionally, provide recommendations pertaining to the opportunities for improvement. Such recommendations may be narrative and/or graphical depending on the topic at hand. They may vary in specificity based on the vendor's needs, the reviewers' user interface design expertise, and the development stage at which the review is conducted.

Expert review findings are typically presented in slideshow or narrative document formats. Slideshow presentations dedicate slides to general and specific topics, each one including an illustration of the user interface element(s) of note, if applicable, a brief description of the strength or opportunity for improvement, and a recommendation for improvement where warranted (see section 6.2 - Sample expert review findings). Narrative documents contain the same basic content but might include somewhat lengthier discussions of particular findings.

The most productive expert reviews typically employ a formal process that ensures comprehensive coverage of the topics of interest. The review should be presented to the development team such that individual findings evoke a constructive response rather than neglect or outright dismissal. Measures to ensure that the expert review findings have a positive influence on an EHR in development or subject to updating include the following:

- Draw upon the expert review results to update the use-related risk assessment. For example, review the expert review results to determine if they have identified additional use errors warranting risk analysis, or suggest modifications to prior estimates of a use error's probability (i.e., likelihood) and the severity of the harm(s) that could arise.

- Add the expert review findings - particularly opportunities for improvement linked to user interface design shortcomings - into a tracking system that assures they will be addressed in some manner, which could include implementing a design change or deciding to keep the design as is. This approach to dealing with expert review findings is akin to how software developers normally track programming "bugs," ensuring that highpriority bugs are fixed before the application's release.

A heuristic analysis is a type of expert review that serves the same basic purpose, but is worth discussion given that it is widely used among software developers. The technique calls for multiple reviewers - typically two to three - to conduct independent reviews according to a common set of evaluation criteria (i.e., design principles such as those presented in section 3 EHR User Interface Design Priniciples). After independently developing a list of problems, the reviewers discuss and reach a consensus regarding the problems and their severity according to applicable criteria such as use-safety, task completion, and usability. In principle, adding more experts is likely to identify a greater proportion of the existing design problems. As such, heuristic analysis is a more rule-based approach to conducting an expert review, which might or might not pay dividends in terms of the end-product's quality. Notably, a heuristic analysis is normally focused on problems as opposed to design strengths. This approach makes it best suited to the evaluation of well-developed user interfaces when the goal is to perfect them, whereas an expert 
review that also focuses on design strengths may be more helpful at an earlier stage of application development.

\subsubsection{Conduct cognitive walkthrough}

It might be impractical to conduct a usability test of a user interface design at the early stage of its development when the EHR might not be functional enough to support such testing. In place of a usability test, developers may engage prospective EHR users in a cognitive walkthrough.

Whereas a formative usability test calls for representative users to interact with a working prototype of an EHR, a cognitive walkthrough requires only static screens, complemented by supporting explanations by the walkthrough administrator (i.e., the administrator "fills in the blanks" left by a lack of design detail and dynamic behavior).

The term walkthrough, just as it is used describe certain types of rehearsals, delineates the process of stepping through a series of activities - a workflow - without actually performing the activities. Participants in an EHR cognitive walkthrough would view certain screens and share their impressions of them. They would also describe how they might act if interacting with a working EHR and discuss the underlying thought processes pertaining to each step of a task (e.g., considering options, performing mental calculations, arriving at decisions).

The EHR conceptual design stage is a good time to conduct a cognitive walkthrough, presuming that the application's user interface is instantiated in the form of static screens only, or perhaps only marginally interactive prototype. Otherwise, it would be more productive to conduct a formative usability test.

A good sample size for a cognitive walkthrough is the same as a formative usability test of an early prototype - perhaps 5-10 individuals. However, the variety of EHR users might warrant a larger sample, albeit one that includes only a few representatives of each type of user.

The technical steps are similar to a formative test as well (see section 2.5.2.1 - Conduct formative usability test). In brief, they may include:

- Determine cognitive walkthrough goals.

- Develop cognitive walkthrough plan.

- Recruit the participants.

- Conduct the cognitive walkthrough sessions.

- Consolidate and analyze the data.

- Document the results.

In practice, cognitive walkthroughs are often conducted in a casual manner as compared to formative usability tests and certainly as compared to a summative usability test. In place of a test plan, the research team may prepare a general guide or even base their efforts on some notes. In 
place of recruiting participants from outside the development organization, insiders who have no prior knowledge of the EHR under development may serve as participants (although recruiting outsiders might still be best practice). As compared to usability tests, walkthroughs can be conducted by a single researcher more readily. However, a two-person research team might still be warranted if the walkthrough covers a significant amount of ground. In place of a document, the research team might choose to write a concise memo to document the results. Alternatively, the team might choose to convey the study findings in a briefing, capturing the major points in meeting notes.

A conceptual walkthrough should be a helpful, convenient exercise rather than a burden to the development process. Still, similar to the way developers should track expert review findings, developers should incorporate the findings from a cognitive walkthrough into the risk management and design optimization efforts.

\subsubsection{Evaluate the preliminary user interface}

When an EHR's user interface has evolved beyond the concept stage and a functional prototype is available, an expert review might still be in order. An expert review takes relatively little time and can be very productive. However, a functional prototype enables a usability test, which is generally considered the optimal way to evaluate a software user interface because it can reveal problems that might go undetected in an expert review. When testing involves a design in formation, it is commonly called a formative usability test. This technique is described below.

\subsubsection{Conduct formative usability test}

Formative usability testing calls for prospective users to perform hands-on tasks with a prototype EHR. As suggested above, the prototype may be fully or partly functional. Indeed, while some portions of the user interface might be quite refined, others might be static or missing altogether. The point is to invite prospective users to interact with the EHR at its early stage of development, when design and software changes are relatively easy to implement, and then at subsequent stages of its development to ensure the design is on the right path toward a successful validation (see section 2.5.4 - Validate the user interface).

Human factors research suggests that even small-scale formative usability tests, involving as few as 5-10 prospective users, generate valuable results. Therefore, it is advisable to conduct multiple small-scale formative usability tests at various points in the development process, rather than one large one at a single point in the development process. Metaphorically speaking, developers are better served by taking the EHR out for many short test drives rather than one long one.

Formative usability test characteristics usually have the following characteristics:

- Test participants represent the intended users. Ideally but with practical exceptions, test participants should not be development organization employees, even if they might have previously served in a role matching that of an intended user (i.e., as a clinician). Rather, participants should be recruited from the organizations that might use the EHR in the future, such as nurses working in large medical practices. 
- Testing is guided by a plan (i.e., protocol) that describes the test goal, test method, test participants, state of EHR development (i.e., the prototype's functional and visual fidelity), hands-on tasks, and data to be collected data. NIST provides a sample usability test plan on its website. ${ }^{4}$

- Test participants may think aloud while interacting with the prototype EHR so that testing personnel can understand the participants' thought processes and identify the root causes of interaction problems that arise.

- Testing reveals the following types of usability-related events, which will indicate where the EHR user interface might require modification to ensure its safe, effective, and satisfying use:

o Use errors - in which a participant uses the EHR in a manner that does not match its intended use and/or could lead to patient injury.

o Close calls - in which a participant almost commits a use error or avoids a use error by relying on her/his vigilance (as opposed to guidance provided by the user interface).

o Difficulties - in which participants struggle to complete a particular task.

- Testing presents multiple opportunities to collect the test participants' opinions about EHR features they like and dislike, thereby identifying opportunities for design improvement.

\footnotetext{
${ }^{4}$ Schumacher, R. M., \& Lowry, S. Z. (2010). NIST guide to the processes approach for improving the usability of electronic health records. National Institute of Standards and Technology.
} 
- Test findings are documented in a document that may include design recommendations. NIST advises that documents include the following contents: ${ }^{5}$

\author{
Executive summary \\ Introduction \\ Method \\ Participants \\ Study design \\ Procedure \\ Test location \\ Test environment \\ Test forms and tools \\ Participant instructions \\ Usability metrics \\ Results \\ Data analysis and documenting \\ Discussion of the findings \\ Appendices \\ Sample recruiting screener \\ Participant demographics \\ Non-disclosure agreement and informed consent form \\ Example moderator's guide \\ System usability scale questionnaire (if used) \\ Incentive receipt and acknowledgment form
}

\title{
2.4.3 Verify the user interface
}

Developers can ensure that EHRs fulfill user interface requirements by verifying a final user interface design. Verification is a matching exercise, often performed and documented using a spreadsheet or other requirements tracking application, that seeks to ensure that there is a design feature or behavior to match each user interface requirement. The exercise serves to ensure that no user interface requirement has been overlooked and/or unmet.

For example, in FDA's parlance regarding medical devices, verification calls for developers to confirm that there is a design output (i.e., user interface design feature or behavior) to match each design input (i.e., user interface design requirement). If there is a requirement is to place a meaningful title at the top of every window and major grouping, verification calls for the inspection of every EHR window and major grouping to ensure that such titles are present.

An EHR's user interface may be considered verified when all user interface requirements have been met by the final (i.e., production-equivalent) user interface design.

\footnotetext{
${ }^{5}$ Schumacher, R., Lowry, S. NISTIR 7742: Customized Common Industry Format Template for Electronic Health Record Usability Testing. 15-November 2010.
} 


\subsubsection{Validate the user interface}

While a developer might produce an EHR that meets established user interface requirements (i.e., is verified), it does not ensure that the intended users will be able to interact with it safely, effectively, not to mention with a modicum of satisfaction. Therefore, design verification is insufficient evidence of user interface design quality. Further evidence of interactive quality is needed, and can be provided by performing a step called validation.

To validate a user interface is to conduct a summative (i.e., final) usability test on a productionequivalent EHR. Such testing differs from formative usability testing because the focus is no longer on identifying design strengths and opportunities for improvement, but rather on determining if users who interact with the EHR in a representative manner are able to complete tasks successfully and without committing use errors that could cause harm.

For example, summative usability testing is the de facto standard way to validate a medical device's use-safety and effectiveness, and both US and international standards on the application of human factors engineering to medical devices call for such testing.

Summative usability test characteristics should have the following characteristics:

- Test participants include representatives of each distinct group of intended users. Most EHR user populations will include multiple distinct user groups.

- The number of test participants is usually greater than the number included in a formative usability test. For example, in the medical device industry, the standard of care for summative usability testing is to include 15 representatives of each distinct user population, suggesting a minimum sample size of 15,30 , and 45 participants for EHR's that will be used by people who individually fit into 1,2 , and 3 distinct user populations (e.g., physicians, registered nurses, and medical assistants), respectively.

- Testing is guided by a plan (i.e., protocol) that describes among other details the test goal, test method, test participants, state of the EHR prototype, hands-on tasks, and data to be collected data. Again, NIST provides a sample usability test plan in NISTIR 7742 Customized Common Industry Format Template for Electronic Health Record Usability Testing.

- Test participants are not directed to think aloud while interacting with the EHR, as they might be instructed to do in a formative usability test, because the information sharing technique can affect how people interact with the EHR in ways that do not serve validation purposes.

- Testing focuses on the riskiest tasks. The objective is to put the high-risk user error mitigations to the test by calling upon test participants to perform the associated tasks and see if the error occurs, or if there are patterns of close calls and difficulties that suggests a greater chance of use errors occurring in real world use. 
- Test personnel seek to identify the root causes of any use errors and patterns of close calls and difficulties based on their observations of test participants performing tasks and associated interviews that usually come at the end of the test when the participant has performed all tasks. A key interview question to pose after a test participant has attempted all hands-on tasks is whether he or she believes the user interface is safe and effective as designed, or needs modification to ensure that it is.

- Test findings are documented in a document that may include design recommendations. Again, NIST provides a sample usability test report in NISTIR 7742 Customized Common Industry Format Template for Electronic Health Record Usability Testing.

With test results and associated analyses in hand, developers should perform a residual risk analysis on any use errors that occurred during the test as well as patterns of close calls and difficulties suggesting an heightened chance of use error. If the residual risk analysis suggests that the EHR is reasonably safe and effective, a developer may conclude that the application has been validated. If the residual risk analysis suggests that the EHR is not reasonably safe and effective, the EHR will require modification and the modifications will require follow-up validation.

\subsubsection{Additional details about validation usability testing}

This section provides expanded guidance on conducting an effective validation usability test of an EHR.

Developers may consider an EHR to be validated when they can make the claim, supported by validation usability test data, that:

- The application is not vulnerable to use errors that could potentially, lead to significant harm (i.e., that the implemented design mitigations effectively protect against use error).

- The intended users could successfully complete tasks that are essential to ensuring patient safety.

The veracity of these claims depends heavily on the quality of the summative usability test and how such testing interrelates with risk management efforts, which in turn interrelates with user research efforts. This explains why it is imperative to apply human factors engineering (aka usability engineering) techniques early and throughout the EHR user interface development process. Starting late or skipping a step not only stands to erode the quality of the user interface, but also jeopardizes a developer's ability to make the above claims.

One approach to summative usability testing, and associated risk management efforts, includes the following steps:

1. Review the use-related risk analysis results to identify high-risk tasks.

2. Write a summative usability test plan that calls for a sample of representative users to attempt to complete high-risk tasks. 
3. Conduct a pilot test (including one or more sessions) to ensure that the test will proceed smoothly without artifacts that could artificially degrade or enhance user performance.

4. Conduct the test sessions.

5. Consolidate and analyze the test data.

6. Perform root cause analysis of user interaction problems (see next section for more detail).

7. Write a summative usability test report.

8. Conduct a residual risk analysis of identified user interaction problems.

9. (If necessary) Modify the EHR to reduce the chance of user interaction problems and conduct follow-on validation testing (potentially focusing only on the modified portions of the EHR). In other words, conduct another iteration of the design-model-test cycle.

10. Update the summative usability test report to include the results of the residual risk analysis; rationales supporting the claim that the EHR is reasonable safe and effective.

As show in in Figure 2-3 and discussed above (Step 9), the process allows for multiple iterations to ensure that all significant, use-related risks have been reduced to an acceptable level in the spirit of preventing harm, and that users are able to complete essential tasks. Simply stated, if a summative usability test reveals that users are committing significant (i.e., safety-related) use errors, the EHR developer should conduct follow-up studies to determine ways to modify the EHR to eliminate or reduce the chance of such occurrences 
Figure 2-3: An illustration of the risk analysis and mitigation process.

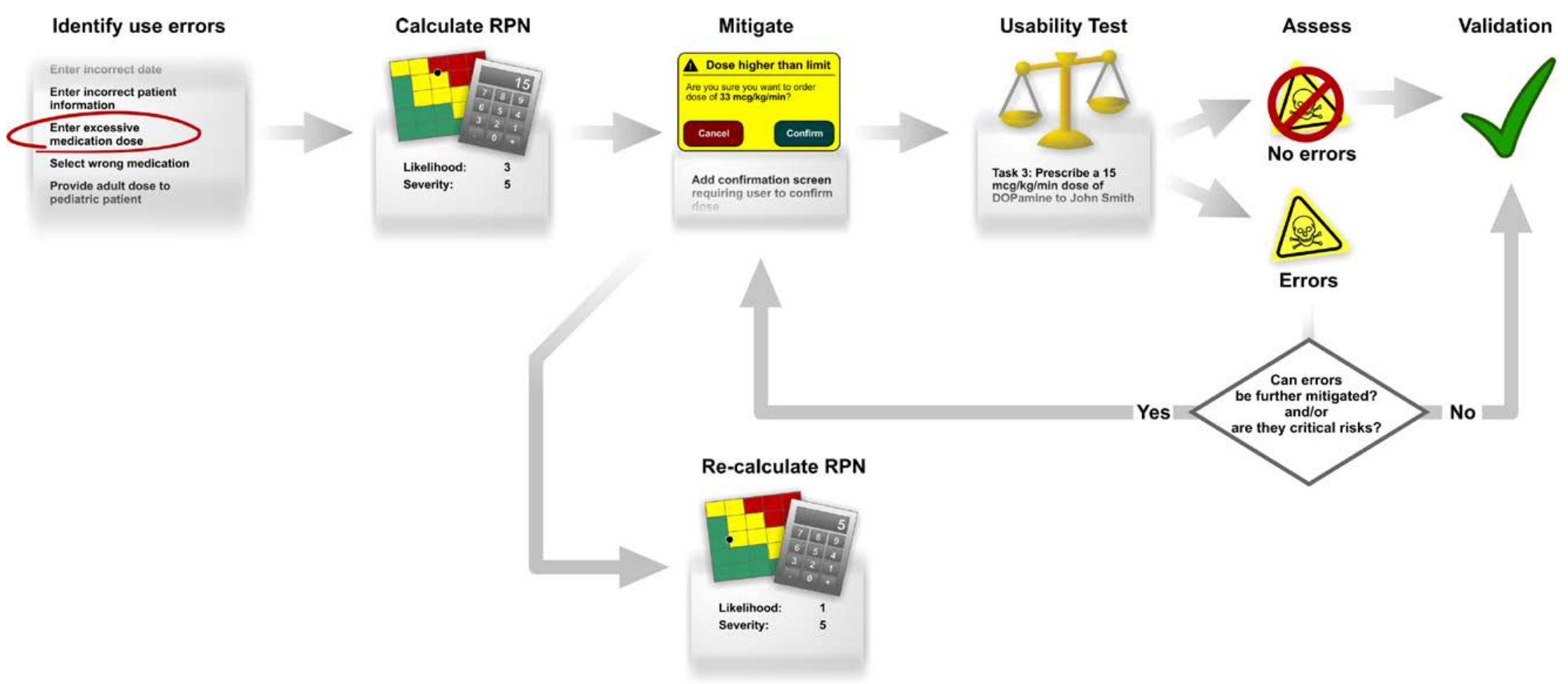




\subsubsection{Recommendations for conducting successful validation usability tests}

Specific validation usability testing recommendations include the following:

- Test participants. Test participants should represent the intended users. Developer employees should not be test participants, even if they have appropriate clinical backgrounds. In cases that the EHR is being validated for use within the USA, the test participants should be USA residents, like, for example, the requirement set forth by FDA for validation usability testing of medical devices.

- EHR configuration. Recognizing that EHRs are often customized for particular institutions and departments within them, developers should choose the most common and complete configuration for validation testing.

- EHR production equivalence. The EHR should be in a production-equivalent form. It should not be a "work-in-progress" that is expected to change before its release.

- Sample data. The EHR should be populated with representative, sample data to enable realistic interactions with the application.

- Test session. Common steps in a usability test sessions are the following:

1. Greet the test participants and have him or her review and complete an informed consent form, which may include statements pertaining to the non-disclosure of confidential information and the use of video recordings and photographs. Inform the test participant that the test is intended to evaluate the EHR, not the participant's skills, work procedures, or clinical expertise.

2. Orient the test participant to the test environment, test personnel, test purpose, and data collection instruments (rating scales, rating criteria). The orientation may include a general introduction to the EHR in a manner that does not constitute training or task assistance.

3. Direct the test participant to perform pre-selected hands-on tasks independently. Depending on the EHR, the number and extent of the tasks can vary widely. It is common for a validation usability test to call upon test participants to perform 1020 separate tasks.

4. After the test participant completes all hands-on tasks, conduct an interview to collect test participant impressions of user interaction problems (e.g., use errors, close calls, and difficulties) and seek feedback about the EHR's overall usesafety and ways to address any particular safety concerns. In certain cases, it is also beneficial to ask follow-up questions after the test participant completes a series of interrelated tasks (see Timing of questions below).

5. Compensate, thank, and dismiss the test participant. 
- Test session duration. A summative usability test usually consumes at least 90 minutes, averages 2 hours, and may extend to 3 hours or longer. Longer test sessions usually include one or more rest periods.

- Test personnel. A summative usability test calls for adherence to a formal plan and requires extensive data collection. As such, it is advantageous to have two people work as a team to administer each test session. Typically, one team member serves as the test administrator, directing the test activities and leading discussions with the test participant. The other team member serves as the data analyst, observing the quality of user interactions with the EHR and recording the associated data (e.g., use errors, close calls, difficulties, anecdotal comments, observations). However, it is possible for one person to conduct test sessions depending on the test's focus, amount of test data, and data collection methods.

- Familiarization period. In cases that a user is likely to take time to become familiar or reacquaint himself or herself with an EHR before using it, it is acceptable to designate time at the beginning of a test session for this purpose. However, test personnel should not dictate a minimum amount of time that should be spent, or how the participant should act. For example, it would be wrong to instruct the participant to study the EHR's use in detail and review the user manual's content. Rather, it is appropriate to state something like this:

"Imagine that it is your first day working in a new hospital unit. The charge nurse expects you to use the unit's EHR independently, and to seek help from a colleague only if you face difficulties you cannot resolve independently. You now have the option to take up to 15 minutes to become familiar with the EHR in the same manner that you might in a real use situation. My colleague and I will be nearby doing other work. Let us know when you feel comfortable proceeding to the handson tasks."

- Thinking aloud. As mentioned earlier, a technique called thinking aloud calls for the test participant to verbalize his or her thoughts while performing a task. It is appropriate during formative usability testing but not during summative usability testing. However, it is acceptable for a summative usability test participant to think aloud spontaneously, just as long as the test administrator does not encourage it.

- Task directions. Task directions are a prompt or instruction provided to a participant at a task's start that describes the high-level task the test participant should perform. It is common to present task directions on a card for the test participant to read aloud. Test personnel may then ask the test participant if he or she understands the task. If there is a lack of understanding, the test administrator may choose to provide minor clarifications regarding the task goal, taking care not to provide any information that could be considered task assistance.

Task directions should be worded in a manner that clarifies the task goal but does not assist test participants by instructing them how to perform the task. Avoid using terms 
that cue users to the proper menu selections or buttons to click. Task directions should use words that all test participants will understand, regardless of age and education level.

Good: Determine if Olivia Green, a 10-month old girl, is growing at a normal rate.

Poor: Olivia Green is a 10-month old girl. Review the growth chart on the "Patient Chart" screen to determine if she is growing at a normal rate.

- Presentation of learning aids. Test personnel should not direct users to access available learning aids (e.g., user manual, online help), noting that no such direction would occur in a real world use scenario. Rather, test participants should access learning aids only on their own initiative and in cases when such aids are normally available.

- Assistance. The test administrator may choose to provide assistance to a test participant when the test participant has (1) reached an impasse such that he or she cannot continue independently, and (2) has passed a practical task time limit. Provide progressive levels of assistance, starting with a minimal assist that will help the participant to "get back on track." Note that providing assistance constitutes a task failure. The reasons to provide any assistance at all, as opposed to stopping the task, are to (1) determine how well the test participant will be able to perform subsequent portions of the task, and (2) provide the user with the necessary, contextual knowledge to proceed with follow-on tasks.

- Assistance threshold. Only assist a test participant with a task when he or she has endeavored to complete the task without assistance for a predetermined period of time (e.g., 10 minutes) that is considered sufficient for even slow performers to complete it. If a participant asks for assistance before the assistance threshold, encourage the participant to persevere with the task.

- Hotline access. It is acceptable to provide test participants access to an actual or simulated hotline (i.e., help line) if such a resource will be available to actual EHR users and the hotline service can be delivered in a "production-equivalent" form. Participants may call a hotline using their cellphone, or a portable phone provided in the test room. Ensure hotline calls are completed using a speakerphone to enable test personnel to monitor and record the call.

- Natural task flow. Do not interrupt the natural flow of a user task by dividing an integrated task into multiple steps or stopping to pose questions or collect ratings, for example. That said, it might be justifiable to divide a large span of activity into chunks that are normally separated by significant amounts of time.

- Timing of questions. Pose essential questions about use errors, close calls, and difficulties - as well as their potential root causes - only at the natural stopping points during the hands-on portion of the test, or after the test participant completes all handson tasks. If debriefing about an event during a post-task interview could bias the participant's subsequent task performance, collect these subjective assessments during the post-test interview. 
- Documenting user interaction problems. Document all user interaction problems in as much detail as possible, including onset conditions and performance influencing factors.

- Identifying root causes. In a post-task or post-session interview regarding use errors, close calls, and difficulties, be sure to ask questions and follow-up questions that generate responses helpful to determining the root causes of the interaction problems. Dissuade users from blaming themselves and encourage them to suggest design-related root causes. Note that the test participants' shared insights might be judged at a later point to be accurate or inaccurate. Indeed, calling on participants to suggest user interface design-related root causes of use error might produce some false positives, but analysts can subsequently separate truth from creative, off-base suggestions.

- Design improvements. Do not solicit design suggestions from participants during a summative usability test. Instead, summative usability testing should focus on collecting data that will determine if it is safe and effective. The only appropriate time to solicit design suggestions is at the end of the test, if the participant says he or she thinks the design needs to change to be safer to use.

- Final use-safety and usability assessment. Near the end of a test after the participant has attempted all hands-on tasks, test personnel should give the test participant the opportunity to comment on the EHR's overall use-safety and indicate if he or she believes there is a need for design modifications (including changes to instructions and training) so ensure its use-safety. Additionally, test personnel may seek test participants' overall impressions of the EHR's usability (unrelated to use-safety) if their responses are of commercial interest.

- Ratings. Test personnel may collect usability ratings focusing on matters such as ease of use and task speed if they are of commercial interest. However, developers should not treat the ratings as evidence of use-safety. Also, to avoid biasing discussions, test personnel should delay the collection of commercial-oriented feedback until all other technical portions of the summative usability test are completed.

- Pleasant, neutral demeanor. Test personnel, and particularly the test administrator, should maintain a pleasant, neutral demeanor during all discussions about the EHR. They should not communicate any pleasure or displeasure regarding the EHR's performance or the test participants' expressed views.

- Training. Test participants should receive training if the EHR developer plans to implement administrative controls that will ensure EHR users receive training before using the EHR. Otherwise, the test should include both trained and untrained participants. Practicality suggests providing consolidated training in a single session as opposed to spreading multiple training sessions over many days or weeks.

- Representative training. Any test participant training should be realistic, taking a realistic amount of time and including the same instruction as a real world training session. Company personnel may deliver the training even if actual training might be delivered by an independent third party. However, the "gold standard" approach is to 
retain an independent party (e.g., one or more clinician educators) to deliver the training if this more closely models how actual training will be delivered.

- Decay period. There should be a decay period between the end of a test participant training session and the ensuing test session. Decay, as short as 1-2 hours might be sufficient, particularly if the training and test sessions are short and the real world gap between training and first use is also short. Longer training and test sessions, and real world cases in which substantial time often passes between training and first use, suggest a longer training decay period (e.g., training on Day 1 and testing on Day 2 ) is appropriate. For pragmatic reasons, it is unusual for decay periods to extend more than a few days.

\subsubsection{Additional details about usability testing}

\subsubsection{Selecting usability test participants}

The quality of a usability test depends in part on the appropriateness of the test participants involved, which is largely under the EHR developer's control. In principal, the usability test plan should include a recruiting script that establishes the characteristics of qualified test participants, which should already be available in the form of user profiles or personas (see section 2.1.1.1 User profiles and section 2.1.1.2 - Personas).

As discussed in section 2.5.5.1 - Selecting usability test participants, usability test participants should bring objectivity to a test session. Therefore, it is generally considered poor practice to engage developer organization representatives as test participants. Even if such participants can be objective from a technical standpoint, their views of a given EHR might be influenced by their organizational affiliation and personal relationships (direct or indirect) with test personnel and other stakeholders. Consequently, their feedback about the EHR could be distorted (overly positive or negative) as compared to the feedback that could be collected from more objective individuals.

Also as discussed in section 2.5.5.1 - Selecting usability test participants, test participants should represent members of an EHR's distinct user populations. It is common practice to engage three to five representatives of each distinct user population in a formative usability test, although larger sample sizes might be warranted, especially when test results will drive major design decisions.

When selecting participants for a summative usability test, test planners should take care to sample each distinct user group equally rather than assemble a group that proportionally mirrors the user population. In other words, if two-thirds of the users are nurses, but there are three additional types of distinct users, nurses should comprise only one quarter of the test participants (e.g., 15 out of 60). In parallel, it is advantageous to select individuals with specific, secondary characteristics to obtain a sample that represents a normal distribution within a particular group of 15 individuals, such as the following:

- Type of care environment (e.g., hospital/unit, clinic, physician's office, long-term care facility) 
- Occupational experience (e.g., years working as a nurse)

- Experience using one or several specific EHRs

\subsubsection{Specifying usability test tasks}

Formative usability tests afford the opportunity to have test participants perform any task of interest to the EHR development team. As such, tasks might be safety-related or not, and task selection might have more to do with which portions of the EHR have been prototyped versus not. Summative usability testing does not afford this degree of freedom. As discussed in section 2.5.4 - Validate the user interface, a summative usability test's purpose is to validate that the intended users can complete safety-related tasks without committing potentially harmful use errors. This requirement automatically constrains the task list, at least the list that pertains to validation, noting that a test may include other tasks about which there is an interest in ensuring a high degree of usability for commercialization sake.

Each task specifications within a summative usability test plan should include the following contents:

- Short task description.

- Test participant prompt (Note that the prompt should exclude content that could instruct the participant how to perform the task).

- List of associated risks (found in use-related risk management documents, such a userelated failure modes and effects analysis). The list can simply reference risk identification numbers included in the risk analysis.

- EHR configuration at the task's starting point.

- Additional information required to complete the task (e.g., a set of patient vital signs requiring manual entry).

- Distractions (if any), such as realistically interrupting a task with a phone call that the test participant would be required to answer.

- Assistance threshold - the point at which a test administrator will offer limited assistance to the test participant to overcome an obstacle to continuing. Note that such assistance should constitute a task failure.

- Summary of expected actions.

- List of anticipated potential use errors. Note that all use errors, anticipated or not, as well as close calls and difficulties should be captured in a data log. 


\subsubsection{Root cause analysis}

This document section provides expanded guidance on how to determine the root causes of use errors and patterns of close calls and difficulties.

It is most productive and accurate to assume that most user interaction problems with an EHR stem from shortcomings in its user interface and support system. Conversely, it is unproductive and arguably naïve to blame the users for interaction problems. As such, summative usability testing specialists abide by the mantra "don't blame the users."

Nonetheless, "to err is human." People play the leading role in the performance of a use error. There is no question that people can be forgetful, careless, clumsy, lazy, confused, and demonstrate many more shortcomings. But just as it is obvious that people can exhibit these shortcomings, it is also obvious that an EHR must be designed defensively to account for these shortcomings.

Developers should be mindful that EHRs are used by different types of people, these people will perform a wide range of types of tasks in various environment and use scenarios, and an EHR may be in use for many years. Logic dictates that some interactions will involve individuals who might not be the strongest performers when it comes to using an EHR, and that the EHR must protect against potentially harmful use errors. Moreover, the EHR's user interface must be free of flaws that would induce even the most gifted users to commit use errors despite their best efforts to perform tasks properly to ensure safety and success.

Moving forward with the viewpoint that user interface design shortcomings, and not users, are the principal cause of use errors, the challenge is to identify the most likely, user interface-related cause of use errors. One approach to root cause analysis (RCA) includes the following steps, some of which may be performed in parallel.

1. Identify provisional root causes. Test personnel, and perhaps other team members familiar with the test results, should have a sense for what might have caused a given user interaction problem. To start the RCA process, it makes sense to document the cause(s) as provisional and subject to change. It might be productive for team members to identify provisional root causes independently and then discuss them enroute to consensus, provisional findings.

2. Analyze anecdotal evidence. Test data should include test participant explanations regarding the cause of use interaction problems. Recognizing that this information might be accurate or inaccurate, analysts should review it to see if it reinforces the provisional root causes(s) or suggests modifying them.

3. Inspect the EHR for user interface design flaws. Arguably, the most productive step in the RCA process is to inspect the EHR's user interface for design flaws. The inspection may be based on the principles provided in section 3-EHR User Interface Design Principles as well as other sources. 
4. Consider other contributing factors. Analysts should also consider whether other factors contributed to the root cause, such as characteristics of other EHRs, which might cause negative transfer of experience that leads to a use error; use environment factors, such as poor interoperability among systems; and test artifacts that induce unnatural user-EHR interactions.

5. Develop a final hypothesis. The final step is to develop a final hypothesis about the user interaction problems root cause(s), informed by the aforementioned analyses. Clearly, the final hypothesis will be the end product of considering evidence and applying professional judgment.

\subsubsection{Document user interaction problems}

This section provides expanded guidance about reporting user interaction problems in a summative usability test report.

A report about a summative usability test of an EHR should thoroughly document the quality of user interactions with the application. Presuming that a developer has followed a user-centered approach to user interface design that has included formative usability testing, the quality of user interactions with a production-equivalent EHR should be quite good. However, there are almost always residual, and potentially minor, problems to report. This is why there is almost always a need for root cause analysis of the problems and an associated residual risk analysis, which might lead to design modifications and follow-up testing. Therefore, it is important to fully document user interaction problems, possibly in a narrative or tabular form, including the following details:

- Problem title. A short description of the problem for convenient reference.

- Associated task. The single or multiple tasks during which the problem occurred.

- Associated risks. Cross references to the use-related risk analysis document and line item (or equivalent) that addresses the problem, indicating its probability, the severity of the potential harm, and any risk reduction measures.

- Potential harm(s). The harm that may arise due to the problem, as already stated in the use-related risk analysis document.

- Problem description. Detailed description of the problem, including the number of test participants who experienced the problem (e.g., committed the use error) and how many times the problem occurred in total.

- Participant-reported root causes. Descriptions of the root causes identified by the participants.

- Root cause analysis. Descriptions of the root causes determined by the usability analysts based on their analysis of test participants' performance and commentary. 
These components of a comprehensive problem document may be augmented later on with the following:

- Residual risk analysis. Document the results of a residual risk analysis of the problem, which may indicate if the EHR's user interface is reasonably safe and effective "as is," or if it needs modification. A description of a potentially effective modification is not necessary, but it may be included in a summative usability test report.

Importantly, even if a single test participant commits an error that could lead to patient harm (e.g., ordering a 10-fold overdose of the intended medication), the user interface should be assessed as a potential cause of the error. Often, the assessment suggests that the user interface could be improved to prevent the use error, and that the improvement should be implemented to bring userelated risk into an acceptable range. After all, once the EHR is released there will be many thousands of opportunities for other clinicians to make the same, harmful error. 


\section{EHR User Interface Design Principles}

\subsection{Introduction}

This document section presents $343 \mathrm{EHR}$ user interface design principles intended to promote good user interface design characteristics and avoid user interface design shortcomings that introduce usability and use-safety problems.

All of the principles are original, although many of them mirror principles developed by other organizations and for software types other than EHRs. After all, while EHRs may have unique characteristics, they also share characteristics with software used by other industries and in other settings.

The research team's principles development process included the following steps:

- Conducted a literature search to identify sources of user interface principles pertaining to diverse products and systems. The approximately 20 sources included standards, textbooks, and websites.

- Starting with a set of over 900 pre-existing principles, assembled a consolidated set of the most applicable principles from the identified literature sources, which subsequently served as inspiration for EHR-specific principles. Many of these pre-existing principles were legitimized by strong empirical evidence, and served as a foundation upon which the research team built the set of EHR-specific principles.

- Wrote EHR-specific principles based on the project research findings, but in some cases inspired by pre-existing principles. Specifically, researchers' qualitative analyses identified evidently good and poor user interface design characteristics. These features were identified through EHR user responses to a Web-based survey, observations of clinicians using EHRs, expert reviews of existing EHRs' user interfaces, and usability tests of EHRs. The researchers then wrote principles.

- Refined the EHR-specific principles based on feedback from multiple reviews by project staff.

The principles prioritize EHR safety and effectiveness over general usability, even though usability is also an important attribute. For example, one principle suggests using consistent terminology, but continues to explain that this step can make an EHR (1) easier to learn to use, and (2) help prevent users from becoming confused by the use of two different terms to describe the same thing. In other cases where the rationale might not be as self-evident, clarification is provided. 
The principles have the following characteristics in common:

- Based on one or more research findings, complemented by research team members' professional judgments.

- None of the principles are based solely on professional judgment.

- Describe a design strategy rather than prescribe a particular software user interface design solution, examples notwithstanding.

- Apply to various types of EHRs rather than just a special class of them.

- Apply to various types of EHR software platforms (e.g., desktop, web, and mobile applications).

- Compatible with various types of design and software development processes.

- Written in plain English as opposed to user interface designer jargon.

- Consistently written in the imperative voice.

The principles are organized into the following categories:

$\begin{array}{lll}\text { Accessibility } & \text { External Consistency } & \text { Status Indication } \\ \text { Affordances } & \text { Feedback } & \text { User Support } \\ \text { Alerts } & \text { Information density } & \text { Visual Design } \\ \text { Clinical Decision Support } & \text { Internal Consistency } & \text { Buttons } \\ \text { Conceptual Model } & \text { Language } & \text { Controls } \\ \text { Content Organization } & \text { Metaphor } & \text { Graphics } \\ \text { Customizability } & \text { Navigation } & \text { Color } \\ \text { Data Entry } & \text { Patient Identification } & \text { Typography } \\ \text { Efficiency } & \text { Search } & \text { Workflow } \\ \text { Error Prevention, } & \text { Security } & \text { Miscellaneous } \\ \text { Detection, and Recovery } & & \end{array}$

Importantly, the principles represent best practices that are likely to enhance a given EHR's usability and use safety. However, they are not intended to be strict requirements and some of them might not be fully applicable to a given EHR. Given the distinct qualities of any given EHR user interface design, EHR developers should determine which principles are applicable to their particular user interface. 


\subsection{Good Design Principles}

\section{Accessibility}

1. Accessibility. Follow standards and principles for software accessibility to the extent feasible and appropriate to the application ${ }^{6}$.

2. Adjustable font size. Enable users to adjust the EHR's font sizes to improve legibility, either by using custom controls in the EHR or using a web browser's built-in "zoom" feature.

3. Display compatibility. Design the EHR in a manner that makes it compatible with different size displays. For example, if a user accesses the EHR on a computer with a smaller than usual display (e.g., a laptop computer display instead of a desktop computer display), critical information and functionality should remain visible and legible.

\section{Affordances}

4. Direct manipulation. Enable users to manipulate onscreen objects directly when this interaction style is likely to make interactions more intuitive, efficient, and error resistant than other interaction styles.

5. Dynamic menu controls. Place informative symbols in consistent locations to control the behavior of dynamic menus (e.g., drop-down menus, roll-over menus, window shade menus). For example, include a downward facing arrow on the left or right side of a control to indicate that clicking on the button will cause additional information to appear.

6. Hidden functions. Avoid implementing key functions in a manner that cannot be readily discovered without reading the user manual (e.g., functions that require the user to right-click on a control, or place the mouse cursor over a control to present hidden information).

\section{Alerts}

7. Alerts. Limit alerts to those that are clinically essential to ensure safe and effective patient care.

8. Alert activation. Ensure that safety-critical alerts cannot be deactivated.

9. Alert prominence. Make alerts visually prominent to help draw the user's attention. When appropriate, place the alert in proximity to the most closely related onscreen contents.

\footnotetext{
${ }^{6}$ For example, the W3C Web Content Accessibility Guidelines, available at http://www.w3.org/WAI/intro/wcag
} 
Figure 3-1: A prominent alert.

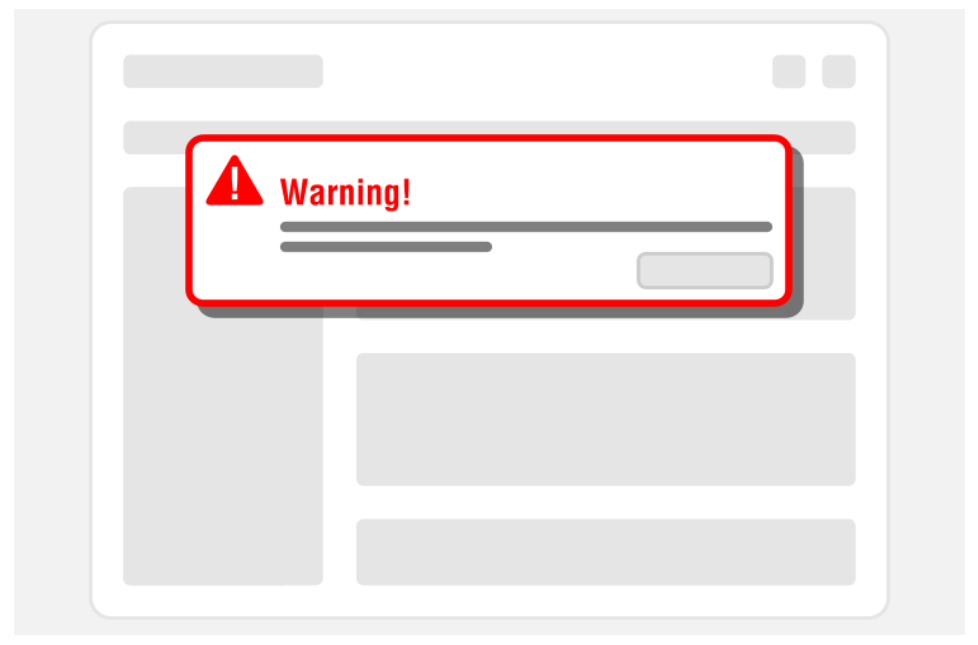

10. Alert acknowledgement. Require users to acknowledge safety-critical alerts, but not necessarily other types of lower-priority alerts.

11. Alert hierarchy. Display alerts in accordance with an importance hierarchy that may give the alerts different appearances, sounds, and behaviors. For example, highest priority alerts might feature a flashing red background, while lowest priority alerts might feature a non-flashing cyan background ${ }^{7}$.

Figure 3-2: An example of alert hierarchy.

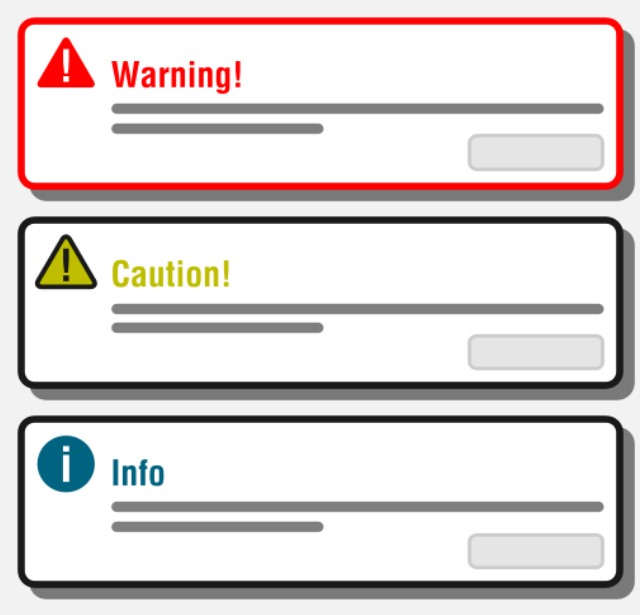

12. Alert timing. Present alerts at the time when they are most relevant to the user's actions. For example, certain alerts might be most useful upon opening patient charts, and others might be useful during plan creation.

${ }^{7}$ IEC 60601-1-8:2006 - Medical electrical equipment -- Part 1-8: General requirements for basic safety and essential performance -- Collateral standard: General requirements, tests and guidance for alarm systems in medical electrical equipment and medical electrical systems 
13. Alert symbols. Avoid using symbols associated with alerts (e.g., a triangle with an exclamation point) for non-alert information. Excessive or incorrect use of alert symbols, some of which might have standardized uses, might cause users to overlook actual alerts.

14. Flashing. Minimize the use of flashing to draw the user's attention to information (e.g., use flashing only for high priority alerts). Flash rates should be no faster than 2 cycles per second. The signal should be visible at least 50 percent of the time ${ }^{8}$.

15. Urgent tasks. Alert users to the need to perform urgent tasks.

\section{Buttons}

16. Button appearance. Make buttons and equivalent, selectable objects visually distinct from other onscreen content.

17. Button label. Ensure that button labels accurately describe the associated function.

18. Button label location. Place button labels on the button's themselves or above the button. Avoid placing button labels below the buttons because the labels are unlikely to be visible during a button press, thereby preventing the user from detecting an erroneous button press.

19. Button names. Generally, label buttons using verb-noun pairs, such as "Add Note" and "Open Chart." The pairing reduces the ambiguities that can arise when the label is a standalone verb or a noun.

20. Button size. Size buttons to facilitate rapid selection using all of the possible pointing devices (e.g., trackpad, mouse, finger), noting that overly small targets can increase selection time and selection errors.

21. Button spacing. Space buttons apart so that their centers are separated by the pixel equivalent of at least 0.5 inches and optimally 0.75 inches or more 9 .

\section{Clinical decision support}

22. Decision support. Provide users with convenient access to the information necessary to make a given clinical decision. For example, the necessary information may be presented on a single screen, or mostly on a single screen with direct links to the rest of the needed information. Avoid partitioning information if it would be more easily acquired and interpreted when presented in an integrated form.

\footnotetext{
${ }^{8}$ ANSI/AAMI HE75:2009/(R)2013 Human factors engineering - Design of medical devices. Section 21.4.10.3.

${ }_{9}^{9}$ ANSI/AAMI HE75:2009/(R)2013 Human factors engineering - Design of medical devices. Section 18.3.2.2.2
} 
23. Reliance on memory. Minimize the number of EHR-specific information (e.g., codes, terms, symbols, special interaction sequences, color meanings) users must remember to interact effectively with the application.

24. Contextual patient details. Persistently display and highlight important patient information, such as disease mechanisms, current medications, and allergies, on patient information displays. Information may be presented in a persistent header, "dashboard," "whiteboard" or similar user interface element that contains key information. Do not permit the patient name to become hidden or displaced by other information due to paging or scrolling, for example.

25. Patient information summary. Present patient information in a form that enables users to quickly develop a comprehensive understanding of the patient's condition, particularly at the start of a new shift (i.e., during shift-turnover). Some use scenarios might warrant displaying the summary in parallel (e.g., in a dedicated window) with other EHR content.

26. Patient history. Enable clinicians to review multiple facets of a patient's medical history at once, serving many possible purposes including orienting clinicians during the shift-turnover process and preparing for rounds.

27. Medication orders. Enable users to order medications with sufficient specificity and precision to cover all potential needs including highly complex ones and those associated with special populations (e.g., neonates, children). For example, a physician might need to order a medication for delivery in one of many possible forms (e.g., injection, liquid, nasal, tablet) and with an unusual degree of precision to treat a patient that is particularly sensitive to a drug (e.g., a low-weight neonate receiving a powerful drug administered based on body weight).

28. Medication range. Display the normal limits (e.g., fluid delivery rate) associated with the delivery of a particular medication. Such information can help users detect a dosing error, such as a ten-fold dosing error that could arise simply by typing an extra "0" in a data entry field.

29. Recent medications. Provide users with a convenient means to view all medications that a patient has taken recently as compared to all prescribed medications. Similarly, indicate which medications the patient was previously prescribed, but has since stopped taking (i.e., discontinued medications).

30. Similar medications. Where appropriate, present lists of similar medications to remind users of different medications that might serve the same purpose as a selected medication and provide certain benefits (e.g., cause fewer side effects).

31. Pending orders. Give users access to a summary of all pending orders for a given patient. Such a summary will enable them to check the accuracy of the orders and possibly edit them to correct mistakes and make necessary additions. 
32. Calculators. Build-in calculators (e.g., determine a drug dose based on patient weight) that that can increase the speed of tasks requiring calculations, and prevent users from having to perform mental calculations that could be subject to error.

Figure 3-3: An example of a dose calculator.

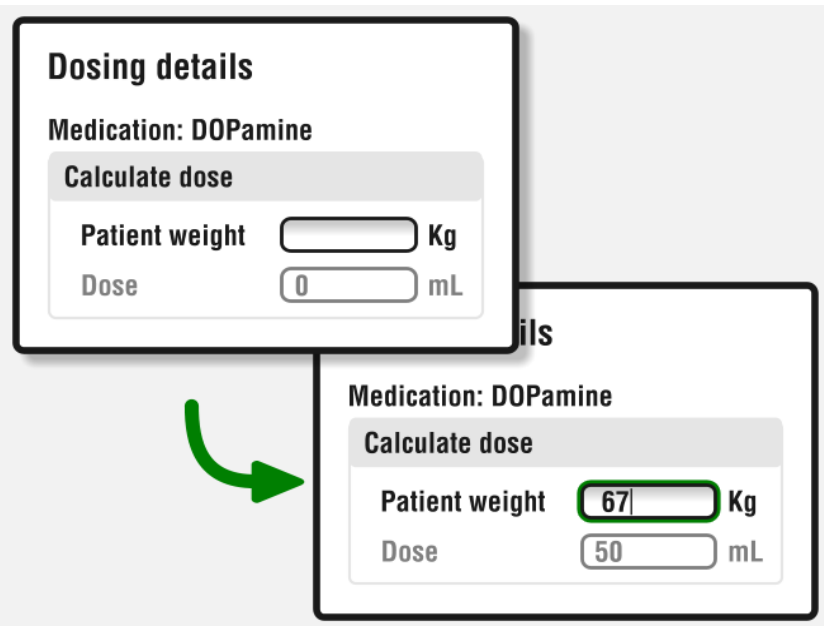

33. Educational materials. Provide access to educational materials that might help the user make clinical decisions and that the user can provide to patients to help them understand certain medical topics.

34. Discontinued medications. Enable users to indicate why medications were discontinued. For example, if a clinician discontinued a patient's medication because it induced an undesirable side effect, other clinicians could use such information to optimize future prescription decisions (e.g., by avoiding medications in the same class)

\section{Conceptual Model}

35. Conceptual model. Establish an overarching EHR organization scheme (i.e., conceptual model) that enables users to form a simple mental model of how to navigate within it and locate specific content. For example, users may be well served by a conceptual model that parses the user interface into a limited number (e.g., 5-7) of major elements.

36. EHR prioritization. Ensure that the conceptual model emphasizes the most important EHR capabilities, such as admitting a patient or charting a patient's progress in a certain type of EHR.

37. Integration. Integrate EHR functions in a manner that gives the user the sense of working within a single, consistently designed application, even if it is actually a set of individual applications that are linked together. 


\section{Content Organization}

38. Information order. Place information in a useful order. For example, within a list of patient visit records, place the report from the most recent visit ahead of the second most recent visit.

39. Information layout. Arrange information in a manner that clarifies functional interrelationships and facilitates efficient visual scanning.

40. Information placement. Place the same information in the same location (on a given screen or within a given type of window, for example) to the extent possible.

41. Information and control duplication. Limit the redundant presentation of information and controls to cases where they serve safety-critical purposes and/or substantially increase task efficiency (i.e., are a major convenience).

42. Unnecessary information. Omit,(i.,e., hide) or de-emphasize (e.g., gray-out) information that is not directly relevant to the user's role, current task, or to other information on the screen.

43. Menu depth. Where possible, limit the menu system depth to three or fewer levels.

44. Similar functions. Avoid providing different functions that users might perceive as being identical to each other. Instead, seek opportunities to consolidate similar functions, thereby simplifying the user interface structure. For example, instead of providing separate tabs for "Patient Conversations" (for documenting telephone calls with patients) and "Patient Visits" (for documenting in-person meetings with patients), provide a single tab "Patient Encounters" that enables users to document both phone and in-person conversations with patients.

45. Data grouping. Present data in logical groups that facilitate patient monitoring and diagnoses (e.g., respiratory ailment, cardiovascular ailment). For example, present on the same screen those parameters that are nearly continuously monitored. 
Figure 3-4: A sample patient summary that presents five groupings of related patient information.

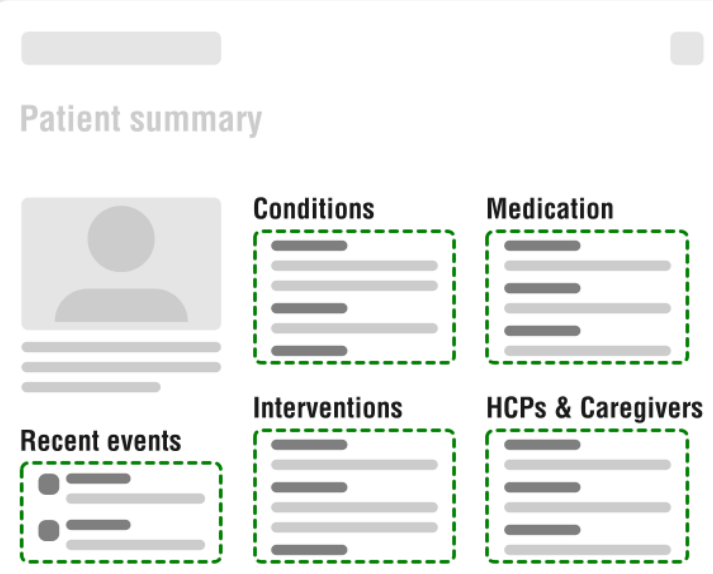

46. Data ordering and filtering. Enable users to control the view of tabulated data, arranging and filtering it to display information in the most useful form.

Figure 3-5: Clicking on a table header sorts the table's data according to selected column.

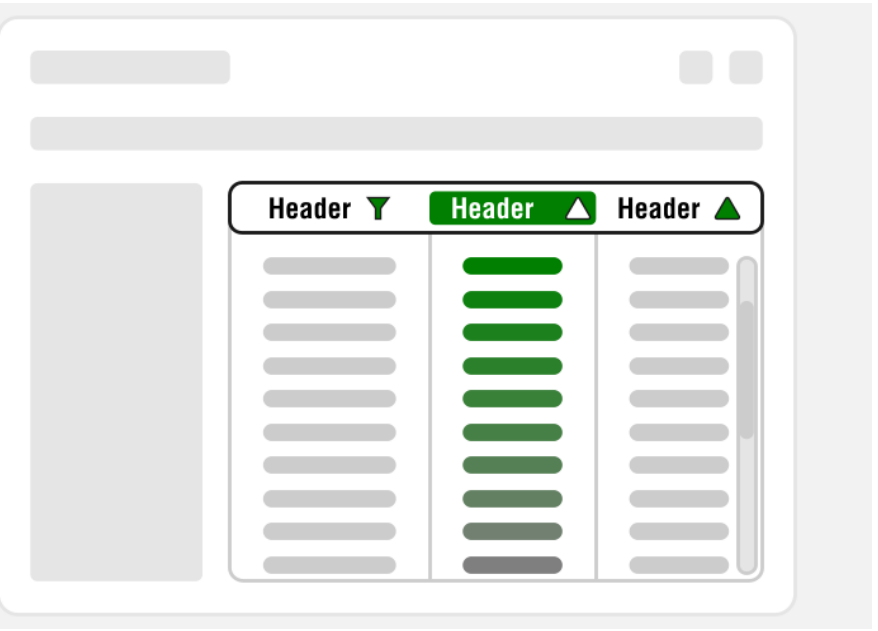

47. Information types. Clearly distinguish between different types of information that could be mistaken for one another (e.g., a flu test vs. a flu shot).

48. Diagnostic data. Provide users with the means to view all diagnostic information (e.g., laboratory results, vital signs, etc.) for a selected patient and the ability to sort or filter them by type, date (e.g., most recent to oldest), and any other important factor.

49. Patient-related content. Clearly distinguish between content that is related to a particular patient and content that is not. 
50. Scrolling. Enable users to scroll within tables and other information presentations that are too large to fit at one time on the screen or within a window, for example. Present scroll controls in a consistent manner throughout the EHR and enable users to scroll either by touching or clicking on the scroll controls or by scrolling the mouse wheel while the mouse cursor hovers over the associated table. Horizontal scrolling should be avoided as much as possible.

Figure 3-6: Samples of scrolling controls for tabular data.
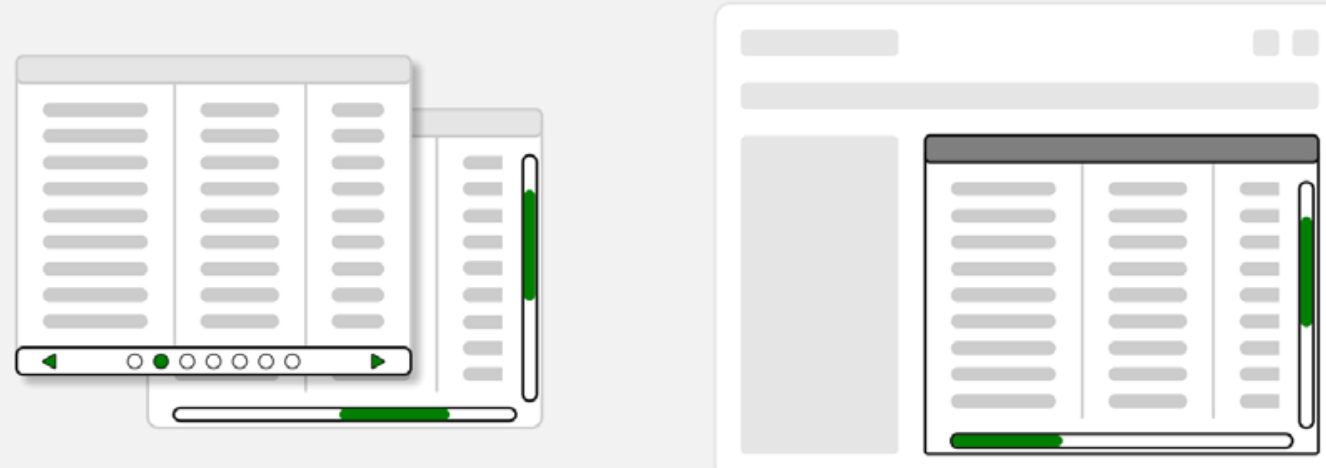

51. Use of labels. Label all major user interface elements (e.g., information groups, windows, pop-up dialog boxes) and special objects (e.g., file cabinet illustration).

Figure 3-7: Examples of panel and pop-up window labels.
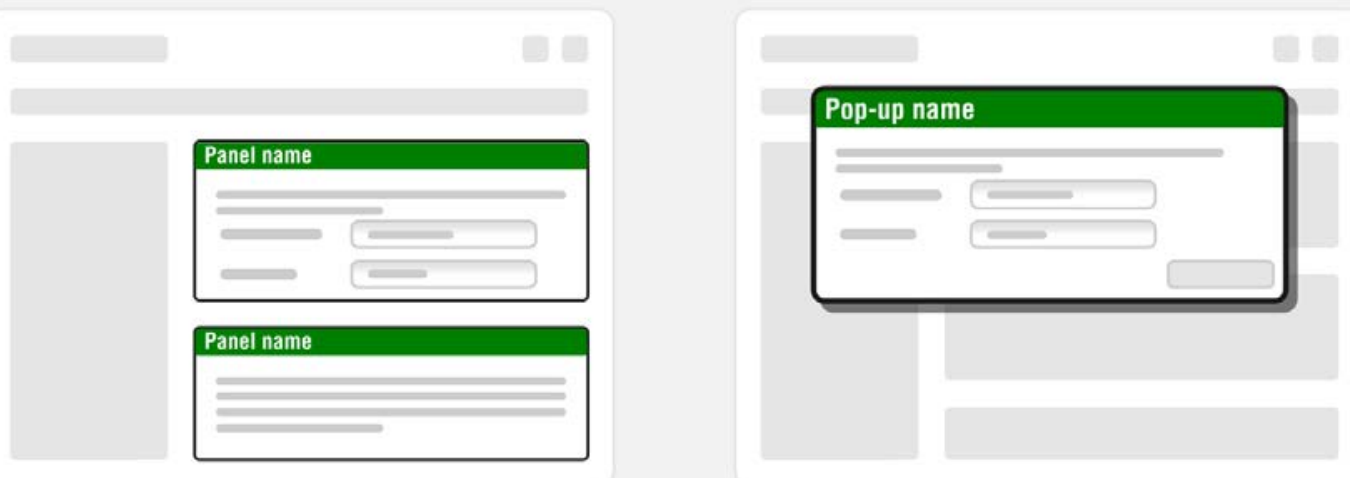

52. Hierarchical labeling. Use hierarchical labeling to reinforce functionally-related or task-related information and controls and to reduce the number of words needed to identify the labeled elements. For example, instead of including the labels "Patient name", "Patient age", and "Patient height", include a heading label "Patient", followed by secondary labels "Name", "Age", and "Height".

53. Label placement. Minimize the distance between labels and the related onscreen elements to ensure that they will be associated.

54. Current parameter values. Differentiate the most recent (i.e., current) parameter value from older ones. 
Figure 3-8: Today's current parameters are highlighted with a green header and thick black outline.

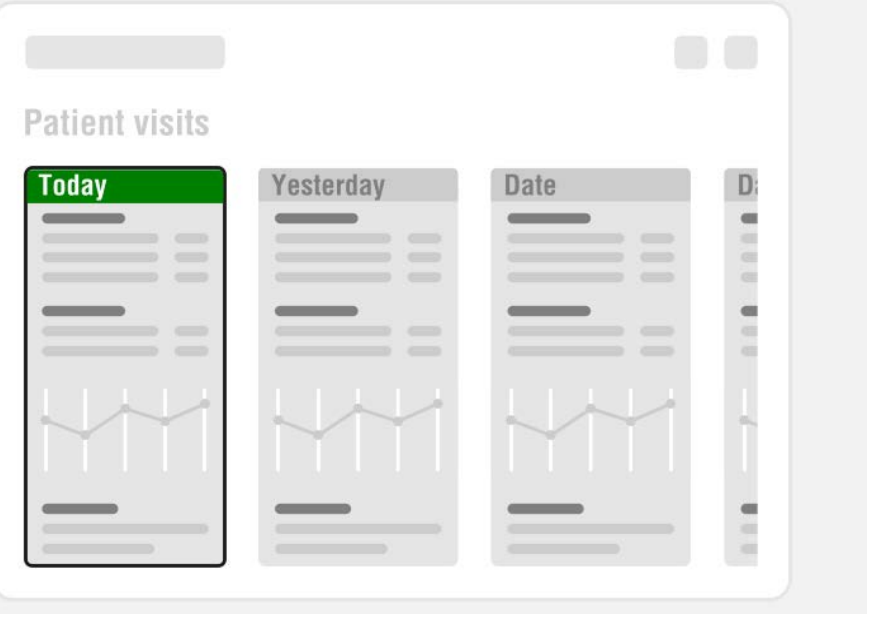

\section{Controls}

55. Control-display relationship. Place controls adjacent to associated information. For example, place a control just below the associated information so that users may immediately recognize the relationship. This physical arrangement also enables users to view the information, rather than block it with their finger, when pressing the control on a touchscreen, for example.

56. Control conspicuity. Make sure that important controls are sufficiently visually conspicuous to stand out from other onscreen elements.

57. Control actions. Controls that have a similar appearance and are grouped together should behave consistently (e.g., each of four controls in a $1 \times 4$ arrangement should evoke pop-up menus, rather than having one of the controls send a message).

58. Single control purpose. Assign a single control a single purpose. Do not vary a single control's function based on the context of use.

59. Antiquated interaction mechanisms. Avoid using user interface mechanisms (i.e., widgets) that are widely considered to be "old-fashioned" and arcane because (1) users might lack familiarity with them (i.e., there would be no positive transfer of experience from other applications), and (2) users might feel disdain for learning to use an application that does not work like other, contemporary applications.

60. Highlighting selections. When a user selects an option that remains on the screen afterward (e.g., 1 out of 10 tabs), change the object's appearance to indicate that it is the one selected. 


\section{Customizability}

61. Customization scope. Enable users to customize EHR functions when there can be significant safety and efficiency improvements, but only when all users will use the same, customized version of the EHR or will log into the system and be the only person to view it in its customized form.

62. Customization constraints. Constrain customization options so that users cannot configure the EHR in a manner that would jeopardize its usability or use safety. For example, if enabling users to customize the colors used for displaying a vital sign, enable users to select only colors that do not produce a low color contrast with the vital sign's

63. Default settings. Enable users to easily return settings to their default values except when the action could cause problems (e.g., initial confusion, longer information search times, erroneous data and control inputs) for other users.

Figure 3-9: An example of "Reset" controls that cause the EHR to revert to its default settings.

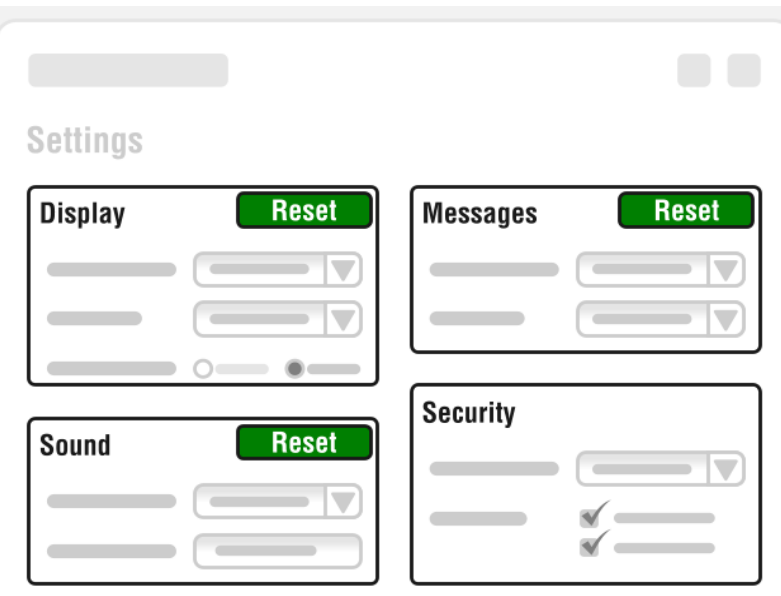

64. Institutional customization. Enable large user groups (e.g., entire hospital units) to customize user interface elements to provide necessary functions and optimize workflows

65. EHR configuration. When an EHR has many distinct user groups, enable users within a group to configure it to best suit their particular needs. Do not make one group of users adapt to a user interface configuration better suited to another user group of users.

66. User accounts. Prevent unauthorized users from customizing the EHR in a manner that will affect other users' interactions with the EHR. For example, enable users to customize settings only for their particular EHR account, but not other users' accounts. 
67. User control. Give users the opportunity to perform tasks in the order that best matches their preferred and appropriate workflow. Make sure that such flexibility does not degrade the users' ability to form a simple mental model of the EHR's organization and workflows.

68. Permanence of customized settings. If the user adjusts a customizable setting on one page in the EHR, apply the setting to other pages in the EHR, thereby avoiding the need to repeat the adjustment.

69. Saving user settings. Avoid requiring users to set their preferences repeatedly. For example, instead of requiring users to manually hide an informational pop-up window multiple times, provide a "Do not show pop-up window" button that will hide the pop-up window permanently.

70. Indication of customizable features. Indicate which aspects of the user interface may be customized.

71. Layout. Consider enabling users to re-arrange on-screen information to best meet their information needs and workflow.

72. Changing units of measure. Enable users to change the units of measure in cases that parameter values might be measured in more than one type of units. For example, enable users to choose to enter patient weight in pounds or kilograms to match the units in which the weight was taken.

73. Resizing. Enable users to resize selected onscreen elements, such as windows, to optimize their view of onscreen content to best suit their needs and preferences. However, an EHR should not enable users to hide or otherwise diminish the conspicuity of essential and safety-critical information, such as alert windows (or equivalent displays).

74. Progressive disclosure. Initially present users with essential information, such as in an overview or summary, and give them the option to access supplemental information upon request. This approach, which gives users control over how much information they view and the pace of presentation, can prevent overloading users with unnecessary information. 
Figure 3-10: The "View more" buttons provide access to additional details; an example of progressive disclosure.

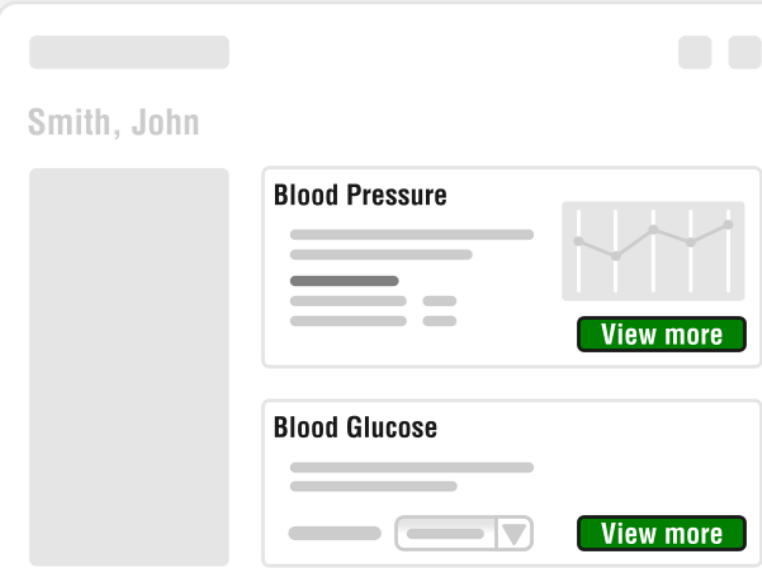

75. Lists. List items in a column rather than in a row to facilitate scanning and differentiate the list from text paragraphs.

Figure 3-11: An example of items presented in a vertical list (left) and in a paragraph format (right).

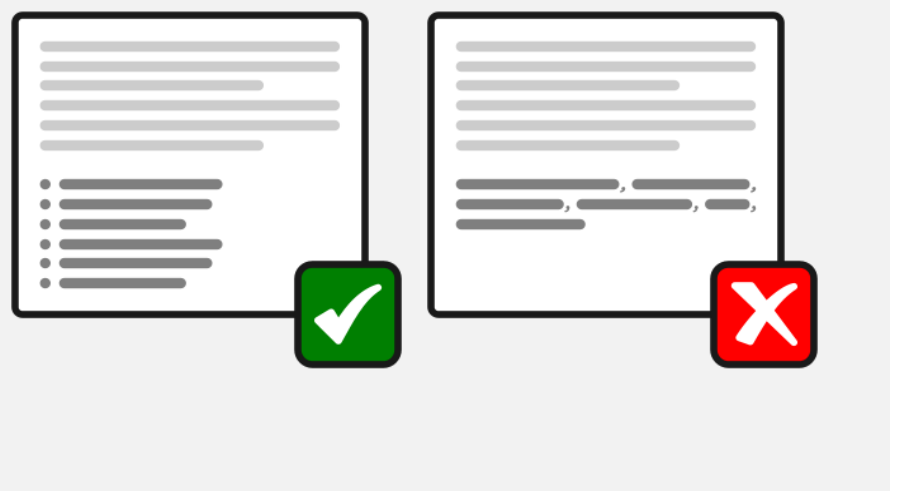

76. Pacing. Let users establish the pace of interaction with the EHR rather than forcing users to perform tasks at a predetermined pace that might exceed their capabilities.

\section{Data Entry}

77. Data input constraints. Constrain the format in which users may enter data. For example, if a numerical entry should be in the 0 to 10.0 range, constrain data entries to be in that range and automatically insert the decimal point when a user enters " 2 " and "7" to specify a dose of "2.7," for example. This technique is called "data masking." 
78. Inputted data validation. Automatically check safety-related, inputted data to ensure it is in the correct format and within an appropriate range. Alternatively, prompt the user to confirm the correctness of inputted values and correct them if necessary. Reject values that are clinically impossible or inappropriate based on patient's characteristics (i.e., patient gender, age) or previously stored values.

Figure 3-12: An example of a data input validation error.

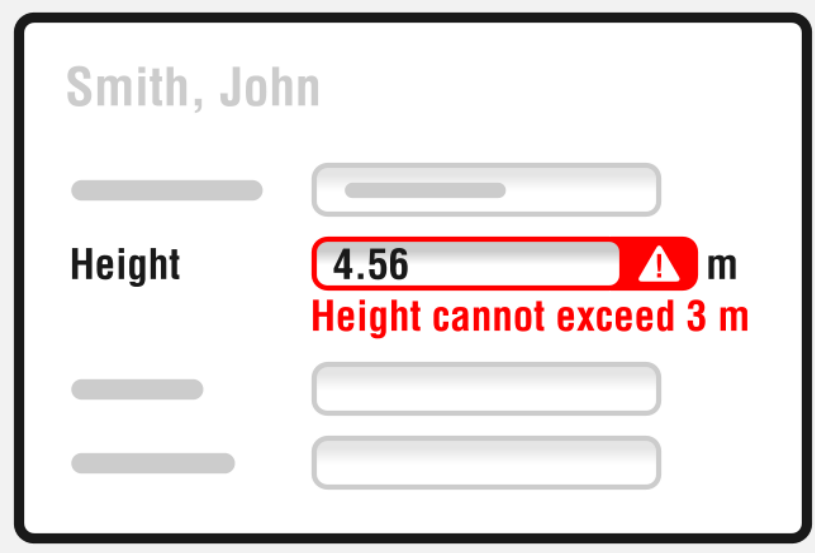

79. Saving changes. Prompt the user to confirm or cancel new data inputs. Also, make the mechanism for saving new inputs explicit (e.g., pressing a "Save" button) as opposed to implicit (e.g., saving by closing a window). Implicit methods for saving inputted data can lead users to question whether or not their inputs were saved.

80. Clear function. Enable users to clear the data entered into a form and start the data entry task again.

81. Unsaved changes. Indicate when the user has made a change (e.g., selected a new setting, entered information into a data field) that still needs to be saved or canceled.

82. Overwriting data. Indicate when new data entries will overwrite existing data.

83. Data protection. Prevent users from editing information considered to be final (e.g., signed off). If users need to modify existing data, require them to "sign off" on their edits and possibly to explain the change.

84. Annotation. Enable users to annotate EHR information as needed to ensure effective patient care. For example, enable users to add a note to vital sign data that might be highly variable for a specific reason that should be communicated to others. 
Figure 3-13: Examples of annotation.

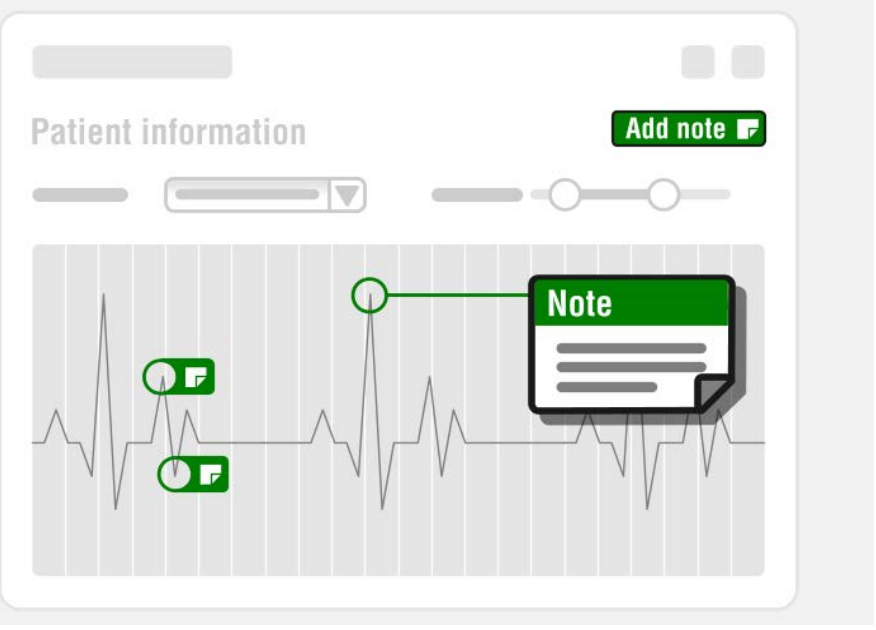

85. Selection versus typing. Where possible, enable users to enter data by selecting from a set of options (e.g., drug names) rather than typing the entry. This approach can accelerate tasks and prevent data entry errors (e.g., typos). The approach also provides users with a sense of the input options as opposed to requiring the user to recall them. In cases where typing allows for improved accuracy or efficiency, enable users to enter data by typing rather than selecting.

Figure 3-14: Examples of selection controls (left) and text entry fields (right).

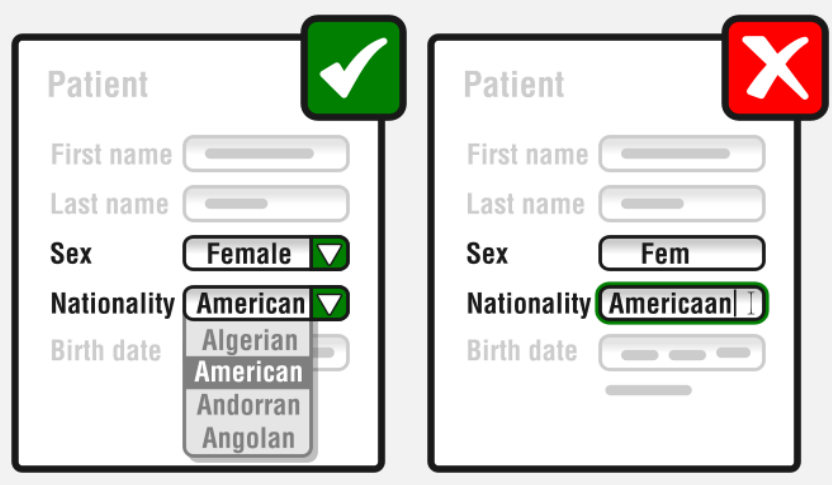

86. Alternative means of data entry. As appropriate, enable users to enter data using various input methods. For example, to the extent made possible by the hardware, enable users to enter data using an on-screen or a physical keyboard.

87. Onscreen keyboards. Use onscreen keyboards that have a conventional layout (e.g., keyboards with an alphabetical or QWERTY layout). Do not require users to type on a keyboard with an unconventional layout because it is likely slow down data entry tasks and increase the chance of data input errors. 
Figure 3-15: An example of a conventional QWERT keyboard layout (top) and a less common Dvorak layout (bottom).

\begin{tabular}{|c|c|c|c|c|c|c|c|c|c|c|c|c|}
\hline Tab & $q$ & w & & & $r$ & $t$ & $y$ & $\mathbf{u}$ & i & 0 & $p$ & 4 \\
\hline Caps & a & & & d & $f$ & g & h & j & k & 1 & & $\leftarrow$ \\
\hline$\uparrow$ & & & $x$ & C & v & b & n & $n$ & & & & \\
\hline 曲 & & ?1 & & & & & & & 1 & & & \\
\hline
\end{tabular}

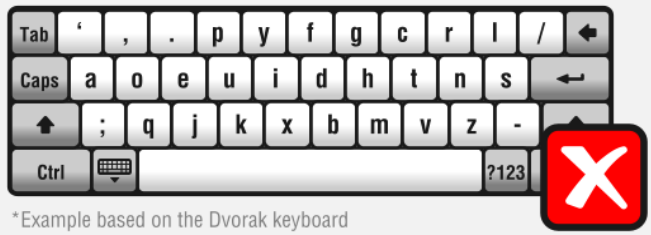

88. Sliders. Provide users with sliders (or equivalent) to change values when this input modality provides sufficiently precise input values. This data entry approach can be faster that typing-in values and less prone to error because typos are impossible.

Figure 3-16: Slider controls used to toggle the alarm status and adjust its volume.

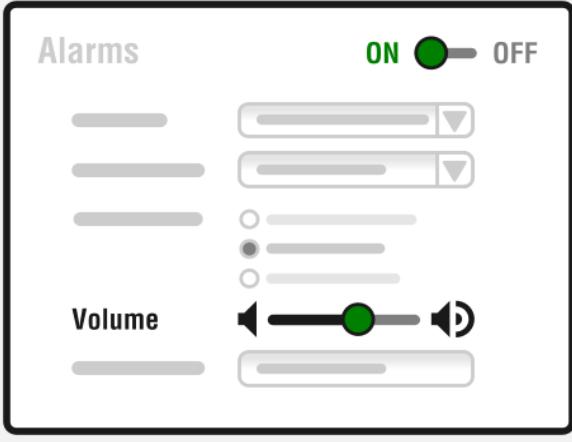

89. Input fields. Make input fields visually distinct from other on-screen elements (e.g., static text fields, instructions, examples).

90. Input field layout. Fit input fields into an alignment grid (system of invisible vertical and horizontal lines) to facilitate content scanning. Do not arrange input fields in what may appear to be a random pattern, presenting multiple input fields in a single row, with spacing dependent on field length alone because such layouts are difficult to scan. Place the fields in a logical order that facilitates a natural workflow. 
Figure 3-17: Examples of a well aligned field layout (left) and a haphazardly arranged layout (right).
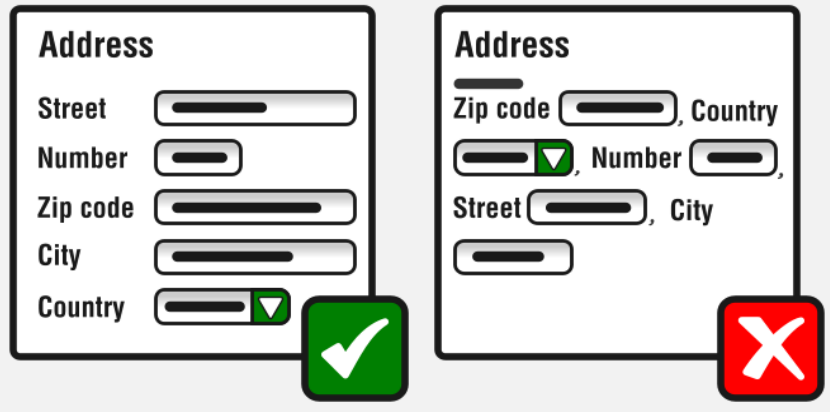

91. Input field size. Make data input fields sufficiently large to contain the necessary amount of information. Do not force users to limit data entries when the limitation can have negative consequences (e.g., an overly terse or incomplete diagnostic comment). Limit the field size to an exact length when the inputted data format is fixed (e.g., 9 numbers such as found in a social security number).

Figure 3-18: An example of well-sized fields (left) and fields that are too small to contain the expected information (right).

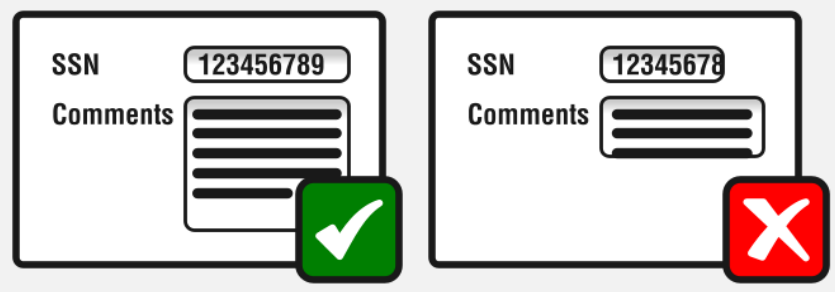


92. Units of measure. Clearly present units of measure adjacent to the associated measures (e.g., vital signs). Avoid mixing units of measure from different systems (e.g., English and Metric) except in cases in which a mixture is conventional. Do not change units automatically, instead require users to make such changes so that they are immediately aware of the change. It is acceptable to present units of measure using common shorthand, such as using "Ibs." as shorthand for "pounds."

Figure 3-19: Sample controls for adjusting weight, height, and temperature units.

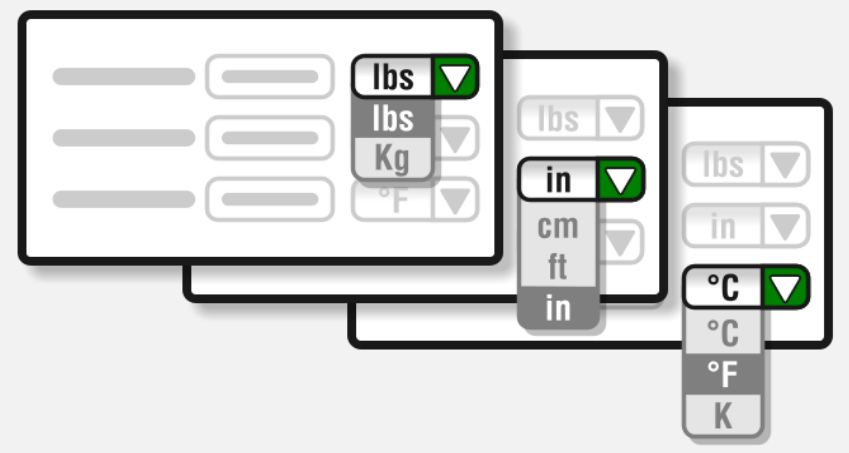

93. Settings. Clearly differentiate between settings where only one option may be selected at a time (e.g., radio buttons) and multiple selections are possible (e.g., check boxes).

94. Data format example. Provide an example of how to format data (e.g., mm-dd-yyyy to indicate the proper format for a birthdate) when a special format is required.

Figure 3-20: A sample format suggestion appearing below the Birth Date field.

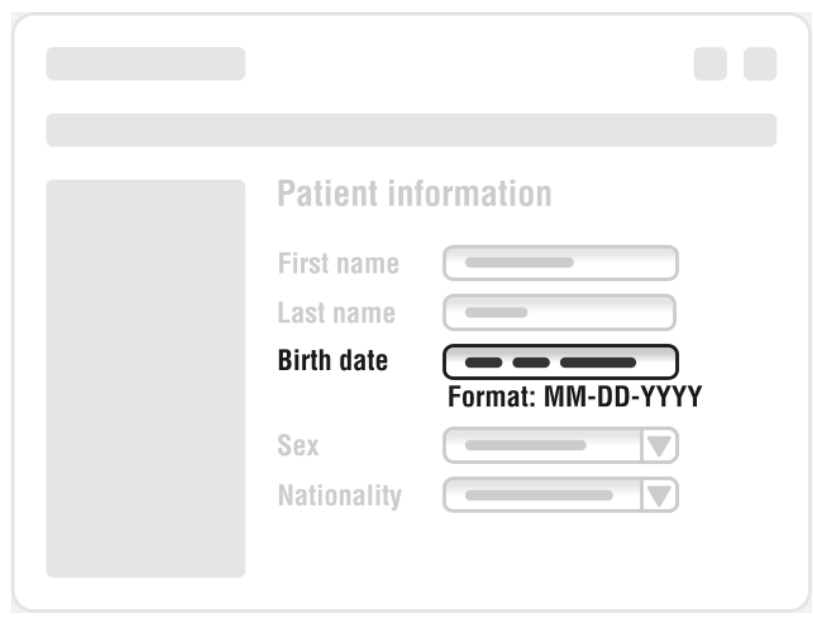

95. Leading and trailing zeroes. Do not require users to enter leading or trailing zeroes when inputting numbers. 
96. Incomplete or missing data. Enable users to indicate, perhaps by annotating a data entry with text, when certain data might be incomplete or unavailable.

97. Missing information. Prompt users to enter any required and missing information before they finish a particular data entry task. For example, a user who is preparing a referral but does not specify a time frame within which a consultation should occur should be reminded to enter the time frame. This principle does not apply to optional information and urgent and emergency use scenarios.

98. Required versus optional fields. Indicate which fields within a data entry form are required (i.e., must be filled-in) versus may be left empty. A common convention is to place an asterisk with required fields, denoting that the asterisk means required in an adjacent key.

99. Copying and pasting information. Enable users to "copy and paste" appropriate information within the EHR, as well as import and export copied information where the capability will reduce user workload and prevent use error.

100. Automated entry of large datasets. Enable automatic data entry where possible, such as enabling the EHR to upload electronic lab report data that automatically enters data for multiple patients.

101. Auto-populate data. Automatically populate forms with data (e.g., patient vital signs) when the data is available electronically. This approach will reduce clinician workload and eliminate data transcription errors.

102. Pre-filling information. Pre-fill information that has already been entered into the system (e.g., patient identifiers) or is known by the system (e.g., city name based on a previously entered zip code).

Figure 3-21: An example of a workflow that utilizes the entered postal code to automatically fill the city field.

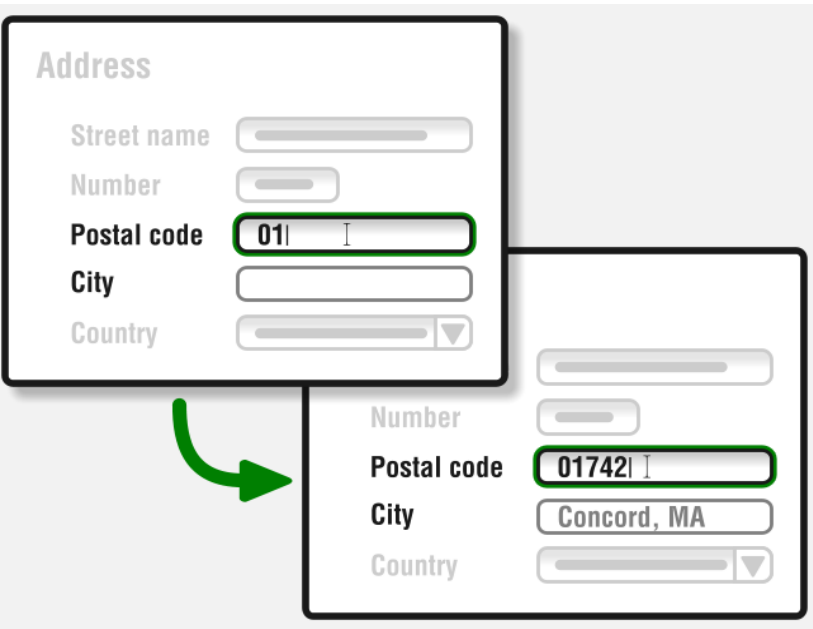


103. Type-ahead. Present data possibilities dynamically as users enter letters into certain fields (e.g., drug name). For example, display a list of all drugs that start with the letters "d" and "o" as soon as the user enters these letters into a drug name field.

Figure 3-22: An example of type-ahead functionality that presents drug names including the entered characters.

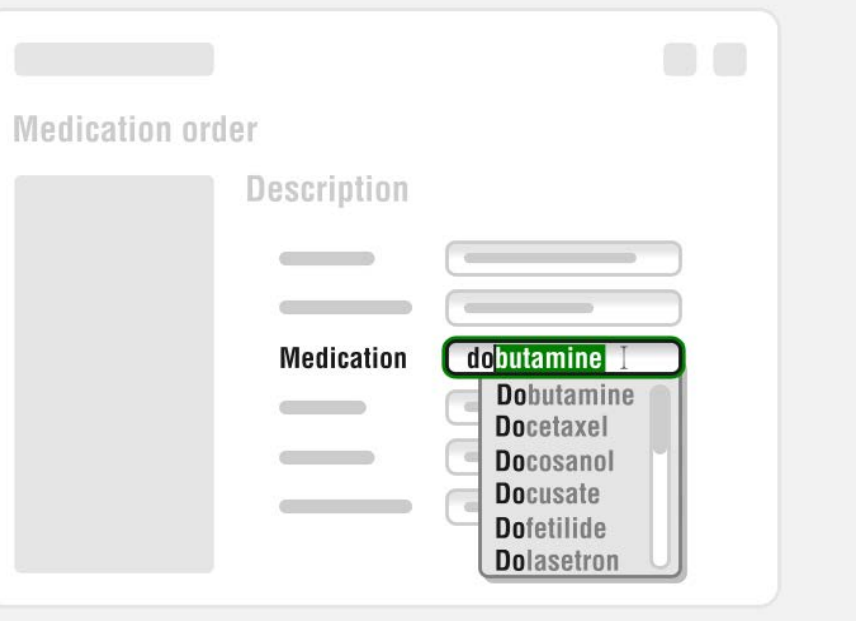

104. Repetitive data entry. Do not require users to enter the same information more than once unless it serves a safety-critical or security-related purpose. The task of reentering data can frustrate users and unnecessarily introduce opportunities for use error.

\section{Efficiency}

105. Improving efficiency. Provide users with the means to complete urgent and frequent tasks rapidly to increase patient safety, limit user workload, and boost productivity. Where necessary, the increased efficiency of high priority tasks may come at the expense of reduced efficiency of low priority and infrequent tasks.

106. Frequent tasks. Ensure that users can perform frequent tasks quickly, compromising the speed of less frequent tasks other than those for which patient safety depends on rapid task performance.

107. Emergency access. Enable users to access the EHR immediately in an emergency, even if they do not have or remember the proper password, for example. Do not lock users out of the system in a use environment in which emergency access might be necessary. Alert the system administrator (or equivalent), as well as other appropriate individuals to the emergency access so that the proper security checks can be initiated.

108. Emergency task performance. Provide users with a direct means to perform emergency tasks, rather than requiring them to first perform non-essential tasks. For example, enable appropriate users to order an emergency medication (e.g., tissue plasminogen activator (t-PA) for ischemic stroke) without having to complete a patient admission form ahead of time. The requirements to first perform relatively low 
importance and non-urgent steps could cause an undesirable delay that could lead to patient harm.

109. Required actions. Provide users with a means to determine required (i.e., pending) actions rapidly. For example, present a prompt guiding the user to perform the next step in a task.

110. Number of steps. Reduce the number of steps required to complete tasks, such as printing a patient chart, by employing default settings and consolidating choices to the fewest screens possible.

111. Keyboard shortcuts. If the EHR will run on a system featuring a physical keyboard, explore opportunities for utilizing keyboard shortcuts that will reduce the time to perform frequently performed actions.

112. Navigation shortcuts. Consider including a shortcut menu that provides direct access to the EHR's most frequently used features (e.g., print, search, medication orders), individuals (e.g., physicians, therapists), and facilities (e.g., referral providers, pharmacies).

113. Previews. Seek opportunities to present previews of information that might eliminate the need for users to navigate to a different page within the EHR. For example, enable users to preview past test results by placing the mouse cursor over a test name, rather than requiring users to click on the test name, go to another screen, and then return to the original screen.

114. Processing time. Minimize the application's processing times, such as data loading times, which occur in the course of performing a task.

115. Pre-set content. Explore opportunities to limit the need for manual typing by providing pre-set content for frequently used functions. For example, include "canned" notes or messages that users can select easily.

116. Operations on multiple selections. Enable users to perform certain functions on multiple selections simultaneously. For example, enable users to select multiple patients simultaneously and allocate them to a particular clinician with a single action.

117. Macros. Consider enabling users to implement "macros" - user-defined combinations of multiple interaction steps - to automate frequently performed actions.

118. Interoperability. Enable the EHR to share data with other systems when it promises to reduce clinicians' workload and data entry and retrieval tasks that otherwise might be vulnerable subject to use error.

119. Integration with billing system. Indicate whether a particular procedure, prescription formulation, or diagnosis is likely to be rejected by a patient's insurance. 
120. Logging in. Limit the effort required to log into the application, considering that users might need to do so dozens of times per day. For example, limit the number of steps required.

121. New patient records. Enable users to create a new patient record rapidly to facilitate urgent or emergency care, such as might be delivered to a newborn baby. Enable nonessential inputs to be omitted, albeit after indicating that some fields have been left blank and confirming it was the user's intention.

122. Recurring orders. Enable users to enter recurring orders, rather than requiring them to re-enter all portions of new but identical orders.

123. Consolidated information. Eliminate or limit the need for users to view many screens to gather the information necessary from multiple screens to perform high-priority tasks. Requiring users to view many, rather than one screen, to acquire the information necessary to accomplish a task can lead users to overlook key information and/or forget it while performing intermediate information collection steps.

\section{Error Prevention, Detection, and Recovery}

124. Safety checks. Build-in safety checks when erroneous or detrimental user actions (prescribing two drugs that have harmful interactions, prescribing a dose that exceeds norms) could lead to harm. For example, alert the user to unusual (i.e., outlier) medication orders.

125. Dependence on memory. Minimize or eliminate the need for users to remember critical numbers (e.g., blood glucose reading)

126. Confirmation. Require the user to confirm or cancel patient safety-related inputs and important, irreversible actions. Do not automatically dismiss the confirmation message after a period of time.

127. Extensive confirmations. In cases where users are about to perform a particularly critical action, consider requiring a confirmation action. For example, require users to re-enter their account password before deleting a patient account or re-entering a dose amount before ordering a medication delivery.

128. Error message appearance. Ensure that error messages have a distinct appearance that reliably draws the users' attention.

129. Error message visibility. Ensure that critical error messages, or direct links to them, will remain visible rather than become covered-up by other information (e.g., overlaying window), contained within a collapsed window, or otherwise not in view.

130. Error message wording. Provide plainly worded messages that adequately explain the error condition without introducing unnecessary details. Do not present error messages using codes (e.g., E-45) that must be memorized or understood by referring to another resource (e.g., instructions for use). Provide clear recovery instructions to 
resolve the given problem, or perhaps instruct the user to contact technical support. State error messages in a neutral tone that does not place blame on the user, but rather provides an objective statement about the error that occurred and how to recover from it.

131. Error recovery. Provide users with the information and mechanisms necessary to recover from use errors immediately upon error detection, and perhaps after considerable time has passed since the error occurred. For example, give users the ability to immediately "undo" an incorrect action and, later on, change a previously submitted order.

132. Undo function. Enable users to undo an action. This capability will enable users to explore (i.e., try-out) application capabilities more freely without concern for taking irreversible steps. It will also enable users to check their work and correct mistakes.

133. Automation. If the EHR can fully resolve an error without user action, then it should not ask the user to do so (e.g., rather than displaying a pop-up error message instructing the user to open the Summary tab, the interface could simply open the Summary tab for the user).

134. Mode sensitivity. Present users with options that are operational mode-appropriate. For example, when prescribing medication to a pediatric patient, present medication dose options that are appropriate to children rather than adults.

135. User roles. Restrict user permissions (i.e., ability to modify and access certain data and functions within the application) based on user roles. For example, do not enable a medical assistant to sign off on a lab result.

136. Use of defaults. Avoid the use of default values when there is a significant risk of harm arising due to inappropriately accepting a default value (e.g., accepting a default medication dose intended for an adult when treating a child). Otherwise, pre-fill fields when there is an especially common input value.

137. Reset. Enable users to return settings to previous and/or default settings except where such actions are contraindicated due to an associated risk.

138. Aged data. Do not display data that has aged to the point that it is no longer pertinent to, or could actually interfere with, safe and effective medical care. Such data could be stored in an archive and be available by request.

139. Updated/synchronized information. Present up-to-date (i.e., time-synchronized) information at a rate that ensures it remains useful and does not conflict substantially with other displays of the same information. At a minimum, indicate when information has aged to the point that it could compromise clinical decisions if actual conditions (e.g., vital signs) have changed. Limit the speed at which values update so that users can read them before they change to a duration matched to the reading task. 
140. Auto-saving. In cases where the user is entering non-safety-related data, automatically save data as the user enters it into the EHR, thereby preventing the deletion of information if users inadvertently navigate to another tab.

141. Data importing. Inform users when they must take action to import data from an external system or website into the EHR. For example, inform users that they must load an external drug library before they can use the EHR's dose calculation settings.

142. Date formatting. Where possible, utilize a date presentation format that explicitly distinguishes between the day and month (e.g., 12-Jun-2013).

143. Duplication. If the user attempts to add data that already exists in the EHR (e.g., adding a duplicate patient chart or medication order), inform the user of the potential duplication.

144. Information sources. Provide a means for users to identify the source of information that was not generated locally (e.g., test results received from a third party or copied from an external source).

145. Information suppression. Indicate when information (e.g., a new message) has been suppressed (i.e. hidden from view) so that users remain aware of it, and provide the means to display it quickly.

146. Medication names. When presenting medication names in the medication order, display both the brand and generic names.

147. Order status. Clearly indicate the status of medication orders (i.e., whether a medication has been ordered or administered)

148. Original data. When it facilitates a task, display original data at the same time that replacement data is entered and until the user confirms the replacement data. For example, when a user reschedules a patient visit, display the original visit date until the user confirms the new date.

149. Patient selection. Require users to load a patient chart by explicitly selecting a patient (e.g., clicking on a patient name in a list of patients). Avoid implicit means of loading a patient chart, such as clicking a "Next" button to load the subsequent patient in the patient list, to prevent inadvertent patient selections.

150. Pre-processed information. Pre-process information for presentation to users in a directly useful form. Do not require users to decode or mentally manipulate the information in complex ways to make it useful. For example, an EHR should perform mathematical calculations for the user when the necessary data is available. As another example, an EHR could present a user with a list of priority tasks distilled from a longer list of pending tasks. Pre-processing information reduces user workload and eliminates opportunities to commit use errors (e.g., incorrect calculations, forgetting to perform a task). Meanwhile, it might be appropriate to present users with raw data 
along with the processed results to ensure a full understanding of the given condition or situation.

151. Recovery timeout period. After users perform destructive actions (e.g., deleting a patient profile), provide a "recovery" period during which they can recover deleted content. For example, enable users to recover deleted messages within the 24 hours following the deletion of a message.

152. Rounding of numbers. Ensure that rounding of numbers cannot result in the loss of valuable precision.

153. AM/PM. When presenting times using a 12-hour format, make the AM/PM indication prominent on clocks and timestamps that serve safety-critical purposes. In particularly critical displays, consider adding reinforcing text, such as "Morning," "Afternoon," "Evening," and "Night."

154. Pop-up location. Pop-up elements (e.g., dialog box) should not cover safety-critical information or information necessary to respond appropriately to the pop-up.

155. Scrolling. Do not require users to scroll to see safety-critical information and controls.

\section{Feedback}

156. Scheduling. Perform automatic checks to prevent schedule conflicts.

157. Use of feedback. Indicate when the EHR is "working" on a task that will not be completed in what users would perceive as an instant. For moderate-length tasks (e.g., a task consuming 5 to 60 seconds), show progress in accurate increments. For longer tasks (e.g., a task consuming 1 minute), consider also displaying the amount of time remaining before the task will be complete. Adjust the time frames to best suit user perceptions, such as those that might be expressed in a usability test.

Figure 3-23: An example of a data import progress indicator. 
158. Confirmation of user actions. Inform the user when their actions have been detected by the EHR. For example, if the user saves changes to a patient chart, provide a clear indication that the changes have been saved successfully.

159. System response time. Ensure that the EHR responds to user inputs rapidly (e.g., within 0.1 second) so that users perceive the response as immediate. Perceptibly immediate system responses will help prevent users from committing double key presses and becoming irritated by delayed system responses. ${ }^{10}$

160. Pop-up windows. Reserve pop-ups for situations requiring prompt user intervention. When presenting pop-up windows that require prompt user intervention, disable other portions of the user interface, so that the user cannot proceed to use the EHR until taking action with the pop-up window.

161. Updated/synchronized information. Indicate when displayed information has been updated dynamically, such as when the EHR receives updated data from a connected patient monitor or when another user updates information in the EHR. For example, highlight newly updated information or present a prompt that informs the user about the information having been updated.

162. Deletions. Provide users with an immediate confirmation when content has been successfully deleted.

163. Interoperability. Provide confirmations that data exported from the EHR has been successfully sent to and/or received by the target system. For example, confirm that a pharmacy successfully received an electronically transmitted prescription.

164. Touchscreen button response. Touchscreen buttons should respond immediately, as perceived by the user, to a touch. The response may be to change appearance as well as actuate the associated function, thereby following a "touch down" control approach. Or, the response may be to change appearance to indicate the "down" state but not actuating the associated function, thereby following a "lift-off" control approach. The latter approach allows users to slide their finger off a button to release it without it actuating the associated function.

165. Use of sound. Do not rely solely on sound alone to provide users with essential information and feedback because some users might mute their computer's speakers or might have a hearing impairment (e.g., conditions that reduce the perceived volume of sounds and make high frequency sounds inaudible) that interfere with their ability to perceive the feedback.

\section{External Consistency}

166. Consistency with for example, medical devices. Explore opportunities to optimize consistency for example, with medical devices that users might use in conjunction with

\footnotetext{
${ }^{10}$ Source: http://www.nngroup.com/articles/response-times-3-important-limits/.
} 
the EHR. For example, arrange vital signs in a manner that is similar to the arrangement on a typical patient monitor.

167. Consistency with legacy EHRs. Avoid non-essential design changes that introduce unnecessary inconsistencies between the EHR and legacy EHRs (i.e., previous versions of the same EHR). Limit the frequency of user interface changes so that the EHR meets users' established expectations, and notify users when changes have been implemented. However, discard suboptimal legacy design features when they become a liability; a threat to EHR use-safety and effectiveness.

168. Cross-platform consistency. If the EHR can be accessed using different types of computers (e.g., desktop computer, laptop computer, tablet, smartphone), ensure that the user interface on each platform is consistent in essential ways with the user interface on the other platforms.

169. Operating system software conventions. Follow software user interface design conventions common to the EHR's operating system (e.g., drag bottom-right corner of a window to re-size it, enable users to use the tab key to jump among data entry fields) to the extent that they help users learn to use EHR in a safe, effective, and efficient manner.

170. Uncommon controls. Avoid using novel or proprietary controls when a conventional control provides similar functionality. Use novel controls only in cases where they enhance the EHR's usability or use-safety. For example, it may be appropriate to implement a specialized control that requires users to confirm a medication order by gesturing a particular pattern on the screen.

171. Keyboard and mouse behavior. Make sure the EHR responds to keyboard and mouse behaviors in a manner that is consistent with established conventions (e.g., right-clicking on the mouse shall cause a contextual menu to appear).

172. Keyboard shortcuts. Avoid using keyboard shortcut combinations that might conflict with established keyboard shortcuts commonly used in other applications.

173. Vital sign color coding. For example, follow medical device conventions for color coding vital sign related information.

174. Date and time. Present the date and time with information for which aging is a relevant factor, such laboratory reports and vital signs. Present time in the conventional, regional format, which might employ a 12-hour clock (whereby mid-afternoon would be 3:00 pm) or a 24-hour clock (whereby mid-afternoon would be 15:00). Ideally, present the date in a manner that explicitly distinguishes between the day and month (e.g., 12Jun-2013). Alternatively, present the date in the conventional, regional format, which is month/day/year (e.g., 12-25-15) in the USA and day/month/year (e.g., 25-12-15) in some European countries, for example. 


\section{Information Density}

175. Content density. Present information and controls more densely on a single screen when it is more helpful to present it in one place rather than many places, eliminating the need for users to search for information and controls on other screens. This objective is particularly important when presenting information in a table, graph, or chart that should be viewed in its totality to identify patterns and anomalies. Even though about 20\%-30\% [1] blank space can improve information readability and a screen's visual appeal, these benefits can be outweighed by the benefit of viewing closely-related information on a single screen.

176. Information spacing. Space information apart to make it easier to read and keep it from looking cluttered. For example, maintain a margin between the side of a window and the information contained within it, and maintain a minimum distance between adjacent icons. Such blank spaces, which are also called padding and gutters usually give a user interface a more pleasing appearance.

\section{Internal Consistency}

177. Consistent controls. Ensure that controls serving similar purposes (e.g., expanding a menu) have a consistent appearance and behavior throughout the EHR.

178. Consistent cursor behavior. Ensure that cursor behaviors (e.g., cursor changes due to roll-overs or mouse clicks) are consistent throughout the EHR. For example, when the user places the cursor over a button, the cursor icon should change from an arrow symbol to a pointing finger symbol.

179. Consistent layout. Ensure that different screens within the application present content in a consistent and appropriately similar arrangement.

180. Consistent icons and graphics. Ensure that icons with the same meaning have an identical appearance.

181. Consistent terms. Use consistent terms in all text-based content including instructions (i.e., prompts), screen titles, data entry field and button labels, and status messages. Consistent terms can reduce the burden on users to learn and recall EHR-specific terms and eliminate uncertainties that might arise from the use of different terms to describe the same thing.

182. Consistent sentence structure. Use consistent sentence structure in all text-based content, including instructions (i.e., prompts), screen titles, data entry field and button labels, and status messages.

183. Information format. Display similar information in a similar manner throughout the user interface. 
184. Transient elements. Display transient elements, such as pop-ups, in a consistent location so that users come to anticipate where they will appear and recognize their functions based on their locations.

\section{Language}

185. Familiar terms. Use terms that are familiar to the intended users and in common use.

186. Distinct terms. Avoid using terms that are synonymous and might be confused for one another (e.g., "Patient Encounters" and "Patient Visits").

187. Jargon. Avoid the use of computer science (i.e., programmer) jargon that is likely to be unfamiliar to the intended users. Conversely, it is acceptable to use medical jargon (e.g., STAT, which means immediately) that will be familiar to the intended users.

188. Invented terms. Avoid or limit the use of invented (i.e., made-up) terms because they can burden users with having to learn and remember them and the terms can be subject to misinterpretation.

189. Slang. Avoid the use of slang (e.g., "pulled chart"), which is distinctly more casual than medical jargon, because some users might not be familiar with it.

190. Abbreviations and acronyms. Limit the use of abbreviations and acronyms to those familiar to, and conventionally used by, the intended users.

191. Definition of abbreviations and acronyms. Provide users with access to definitions of the abbreviations and acronyms used by the EHR.

192. Truncation. Do not truncate information, such as drug names and patient names.

193. Concise wording. Write concise text passages to accelerate information acquisition by minimizing the amount of reading required.

194. Names. Ensure that names, such as those used to describe laboratory tests, are sufficiently descriptive and distinctive so as to prevent confusion and selection errors.

195. Ambiguous status indications. Avoid using generic labels that might not clearly convey the intended information. For example, present the label "Order: Not delivered" instead of "Order: No", which might be misinterpreted by the user.

196. Localization. Enable users to switch the EHR's language to match their preference among the available, regionally-appropriate options.

197. Paragraph breaks. Separate what otherwise would be large text blocks into smaller ones using paragraph breaks (e.g., a breaks every 3-4 sentences) to enhance text readability. Users are prone to only scan or skip reading long paragraphs. 
198. Use of term "exit". Limit the use of the term "exit" to cases when the EHR will close in response to a control option labeled "exit." Using the term for other purposes can raise concern among users that they will exit the application altogether, as opposed to existing a particular window, for example.

\section{Metaphor}

199. Use of metaphor. Employ metaphorical user interface features (e.g., tabbed pages, patient chart, calendar, address book, dashboard) when most users will have real world experience that makes use of a given metaphor advantageous, such as making certain interactions (e.g., moving to a new page) more intuitive.

200. Metaphorical consistency. Apply metaphors consistently and completely so that user interface behaviors (e.g., how the pages turn within a multiple page document) are predictable, complemented by appropriate, metaphor-compatible terminology. For example, it would be appropriate to open and close a drawer in conjunction with filing documents in a metaphorical cabinet, but it would not be appropriate to open and close metaphorical folder tabs.

\section{Miscellaneous}

201. Calendar - level of detail. Enable users to switch between daily, weekly, and monthly within calendar-type features.

202. Contact information. Provide users with a convenient means (e.g., phone number, email address, text message box) to communicate with other professionals (e.g., physician listed in a referral, administrative assistant who schedules patient visits).

203. Information retrieval time. Minimize the time the system retrieve information, thereby accelerating user tasks. Tolerable wait times can be as short as two seconds before users become impatient and possible cancel the information request.[1]

204. Patient focus. To the maximum extent possible, enable clinicians to focus attention on the patient instead of the EHR at key stages of a patient encounter.

\section{Navigation}

205. Calendar - return to current day. Provide a means to return immediately to the current day within a calendar.

206. Navigation control location. Place screen navigation controls (e.g., " $X$ " button that closes an open window) in consistent locations (e.g., upper-right or lower-right corners).

207. Navigation control prominence. Make screen navigation controls (e.g., " $X$ " button that closes an open window) prominent. 
208. Main screen. As appropriate, enable users to form an accurate mental model of EHR user interface, as well as rapidly access major EHR features, by establishing a "home screen," which might also be called a "resting screen" or "initial display."

209. Returning to main screen. Provide users with a direct means to return to the top of the user interface structure (i.e., the home screen). For example, enable users to return to the home screen by clicking on an application logo in the upper left corner of the screen.

210. Backing up. Enable users to go back a step except when a previous step must be rendered irreversible for logical reasons. Provide a clear means to return to the previously viewed screen (e.g., a dedicated "Go Back" button or the web browser's built-in back button, if the EHR is web-based).

211. Browser back button behavior. If the EHR is accessed using a web browser, ensure that the browser's back button behaves as expected (i.e., navigating back to the last viewed screen).

212. Menu item order. Navigational menus should be arranged in a logical order based on the expected task-flow and/or frequency of use of the included menu items.

213. Link-destination labeling. Match the names of text links and destination page titles to facilitate user interface navigation and to enable users to form an accurate mental model.

214. Screen titles. Add a meaningful, visually dominant title to every screen to help users recognize the nature of the displayed information and help them form a clear association between selected controls/options and displayed information.

215. Relative location within user interface structure. Indicate the currently viewed page's location within the overall user interface structure by displaying a "breadcrumb" or similar cue.

216. Functional grouping of menu and content. Visually strengthen the link between navigation buttons and content areas (e.g., use a tab metaphor, animate the transition between views to emphasize that the content in the main window originates from the view icons).

217. Pagination. Online documents should be paginated (e.g., Page 6 of 30) so that users can determine their place in the documents and, in lieu of hyperlinks, find information of interest after reading a table of contents or index based on page number.

218. Tabbing among data entry fields. Enable users to tab (i.e., press the tab key) to jump among required fields within a data entry form in a logical order. Within a form that generally appears to have rows and columns, highlight input fields starting at the topleft, moving to the right on the given row, and then progressing left side of the next row 
down. Do not tab to protected or inactive input fields that are not subject to input or modification.

\section{Patient Identification}

219. Patient identification. Provide multiple, parallel, and salient means to correctly identify a patient in the system. The need for parallel means stems from the potential for patients to have the same name (e.g., father and son) or the same birthdates, which could lead users to select the wrong patient based on one variable (e.g., name). Possible identifiers include the patient's name, unique identifying number, age, gender, weight, location, and photograph. Such information might be most conspicuous if placed at the top of the display or pertinent task window, for example.

220. Patient name. Place the patient's name prominently (e.g., top-left corner) on screens or windows presenting information about a specific patient. .

221. Patient overview. Present a summary of patient information, in addition to the patient's name, when the added information will help identify the patient and/or immediately bring key information (e.g., last office visit, last diagnosis, and allergy) to the user's attention.

Figure 3-24: A sample patient overview layout.
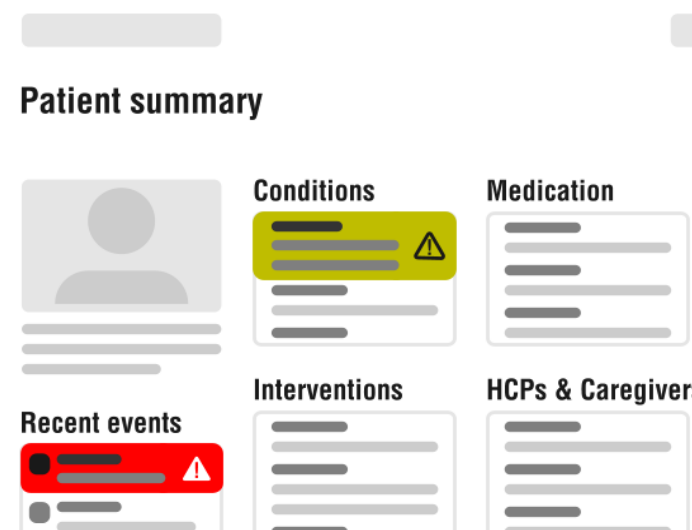

Interventions

HCPs \& Caregivers
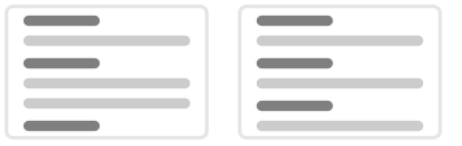

222. Patient photograph. Present a patient photograph with other patient information when the feature will help ensure reliable patient identification or enhance bedside manner, for example.

223. Linking inputted data and patients. Ensure that there is a strong association between data entry controls and the associated patient(s). For example, users should be able to clearly associate a patient name with a scheduled appointment. 


\section{Search}

224. Search availability. Enable users to search for information (e.g., a patient chart) when the capability is likely to make information acquisition tasks more productive. Provide multiple ways to search (e.g., by patient's first name, by patient's last name, by date-ofbirth) to help ensure a successful search.

225. Default search mode. By default, utilize an inclusive search algorithm that searches for any (rather than all) of the search terms entered by the user. Enable users to switch to an exclusive search mode (which finds only results that include all search terms entered by the user) by modifying the search syntax (e.g., including "and" between search terms) or by selecting the preferred search setting from a dropdown list.

226. Patient search. Enable users to access patient information using multiple logical methods (e.g., first name, last name, patient hospital ID, admission date, date of birth).

227. Search field. Visually distinguish the search field from other fields in the application. For example, utilize shape coding, color coding, or labeling to help users notice and identify the search field.

228. Pre-population of search boxes. Prepopulate search fields with search results to accelerate the search task and assist users with searches when they are unsure how the item sought is spelled.

229. Special characters. Ensure that search fields accept special characters (e.g., apostrophes, symbols, accented letters, etc.).

230. Suggestions. Provide suggestions for similar searches (e.g., searching for a medication using an alternative name) or searches using different spellings (e.g., if the software detects that the user misspelled the search term).

231. Similar search terms. Present search results for terms that are similar to the search terms entered by the user (i.e., synonyms). For example, enable users to search for a bone density scan using the phrases "DEXA scan" or "bone density scan", and to search for a thyroid stimulating hormone test by searching for either "TSH" or "thyroid stimulating hormone.

232. Specificity. Enable users to enter different types of information into search fields, rather than requiring them to enter only one specific type of information. For example, enable users to search for different types of orders without having to first specify the type of order.

233. Searched content. Enable users to search for content that exists in the EHR's database, as well as in documents that are accessible through the EHR (for example, text files, PDF documents, and spreadsheets).

234. Number of search results. Provide a clear indication of the total number of search results. 
235. Filtering. Enable users to filter search results by adjusting search settings after performing an initial search.

\section{Security}

236. Screen locking. Enable users to lock screens when there is a safety or privacy-related risk associated with unauthorized access to the EHR. Also, consider providing a means to access EHR functionality in an urgent situation or emergency without entering a password.

237. Overrides. Enable users to override system protections when there are use cases that call for them to have full freedom to perform certain tasks, such as ordering a medication that might normally be contraindicated. In such cases, ensure that the user has the credentials to override the system and require the user to confirm the exception and enter a justification.

238. Unauthorized functions. Hide functions from users who are not authorized to use them, or alter their appearance in a manner that indicates that the functions are unavailable (e.g., display them grayed-out).

239. Privacy. Incorporate features that help to ensure the privacy of patient information, thereby satisfying governmental and institutional rules (e.g., HIPAA, local hospital policies) and user preferences.

\section{Status Indication}

240. Status indication. Keep the user informed in a timely manner and by appropriate means (e.g., alerts, pop-up messages, status bar, activity tracking window) about the system's status (e.g., the system's readiness to receive inputs, the time remaining to complete a task, system connectivity, data transfer progress).

241. Status description. Provide unambiguous status indications. For example, regarding the status of a lab test order, use terms such as "pending" or "completed" rather than "no" and "yes."

242. Mode indication. Conspicuously and persistently indicate the EHR's operational mode (or equivalent operational state) when there is more than one (e.g., adult patient mode versus pediatric patient mode).

243. Multi-user collaboration. If the EHR can be used by multiple users simultaneously, provide an indication when other users are viewing or editing the displayed information.

\section{User Support}

244. Non-reliance on memory. Provide users with the information needed to make decisions and take action. Prompt users to perform necessary tasks. Do not rely on them to recall information and to take critical actions. 
245. Procedural guidance. Guide users through unfamiliar, difficult to remember, and/or infrequently performed procedures (e.g., lead users through a linear sequence of steps).

246. Prompts. Provide detailed task prompts when there is only one or a small number of tasks that users may perform at a given stage of user interaction with the EHR. Provide a more general prompt if the task options are many and yet it will help to inform he user that the system is waiting for them to take action. Place prompts in a consistent location on the screen where users will become accustomed to looking for instruction, or in close proximity to the pertinent onscreen content, such as information contained in a window. Prompts should remain on the screen until the user takes appropriate action.

247. Contextual support. Where possible, provide guidance within the user interface itself, rather than requiring users to access a separate learning aid.

248. Online help. Provide online help regarding topics for which there might be a need for immediate and concise guidance to complete a task. Limit online help to content that will help users understand basic operations and complete key tasks safely and effectively. Separately, provide users with access to a complete source of guidance (e.g., user manual) that might be necessary for users to develop a deeper understanding of certain EHR features of particular interest. Where possible, offer users context-sensitive help, taking them initially to online help content that is likely to be of interest given the task underway at the time the user seeks online help. Give users a direct means to return to a prior task after consulting the online help.

249. Access to technical support. Provide users with the information necessary (e.g., hotline phone number, email address) to contact technical support.

250. Tooltips. Complement icons and buttons with tooltips (or an equivalent) that explain the feature.

251. Clarifications. Augment screen titles, prompts, and similar affordances with even more clarifying information when necessary, and perhaps upon request, to ensure that users understand how to perform tasks.

252. Context. Provide users with a sense of the task at hand by various means, such as the series of recent selections (i.e., a "bread crumb" trail or action history) or a checklist showing the steps completed versus remaining.

253. Failure indications. Indicate when the EHR has failed or its functional capabilities have compromised.

254. Links to external resources. Indicate when a control action will take the user outside of the application, such as to a separate website that provides background information about a medical condition. In addition, provide a clear means for returning to the application from the external resources. 
255. Meaningful text links. Use meaningful text as links (aka hyperlinks) so that users understand where they will go (i.e., what they will see at the destination point) when they click on the link.

256. User feedback. Provide users with the means to provide feedback to the EHR system administrator (or equivalent) regarding what they judge to be hazardous and/or ineffective EHR characteristics and software "bugs."

257. Diagnosis code search. When appropriate, provide clinicians with diagnosis code suggestions based on the patient's problem list and order placed. For example, if a clinician creates an order for Metformin, suggest diabetes-related ICD codes.

\section{Visual Design - Color}

258. Use of color. Use color in a meaningful way, such as to code certain information (e.g., red $=$ abnormal lab value) and to draw attention (e.g., display alert information as black text on a light orange background). Color code misinterpretations are more likely when there are more than eight colors with coded meanings that users must recall.[1]

259. Accommodating color blindness. Do not rely on color alone to draw attention to or visually differentiate critical information, particularly because some users might have impaired color vision (e.g., Deuteranope, which makes red and green objects look similarly gray). For example, not only display abnormal test result in red text and numbers, but also display them in reverse video (e.g., white numbers against a red background).

260. Use of conventional colors. Use colors in a manner that matches user population conventions (e.g., use red in the USA to indicate a dangerous situation or condition).

261. Use of alarm-related colors. Use alarm-related colors (e.g., red and yellow) only for critical, urgent indications. Ensure that the color use matches applicable standards for alarms and medical applications.

262. Color saturation. Avoid using highly saturated (pure hues rather than colors that include some amount of gray) color backgrounds except where they serve a definitive purpose, such as drawing the user's attention to safety-critical information.

263. Visual contrast. Ensure that onscreen content (e.g., text, numbers, icons, lines) contrast appropriately with their backgrounds. For example, very dark content (e.g., dark blue or black text) contrasts well with a light background (e.g., white or light gray data entry field). Medium brightness content (e.g., text, numbers, waveforms) and their backgrounds do not afford good contrast with each other, even when the colors vary. Contrast between foreground contents and their backgrounds should be at least 6:1 and ideally closer to 10:1.

264. Highlighting. Highlight important information (e.g., make it bigger, color it, separate it from other information) so that it draws the user's attention. Lacking such highlighting, 
users might overlook important information that is embedded within less important information.

265. Highlighting changes. Visually highlight recent changes to information sets, such as medication orders, diagnoses, and appointments.

\section{Visual Design - General}

266. Aesthetics. Follow fundamental rules of design pertaining to screen layout, proportion, color, typography, and other visual design attributes to produce an aesthetically pleasing user interface. Note that appropriately aesthetic solutions tend to be more usable than those that look unappealing, and can increase user satisfaction with an EHR. However, do not compromise the safety and effectiveness of the user interface design for the sake of enhanced aesthetics.

267. Unnecessary decoration. Do not add decorative visual features and effects (static or dynamic) that could compromise the EHR's use-safety, utility, and usability.

268. Functional grouping. Visually demarcate functionally related information (e.g., separate one block of related information from another using a borderline or blank space).

269. Information alignment. Place information in a format that facilitates rapid scanning. For example, left-justify the labels in one column (or right-justify them if the label string lengths vary substantially) and left-justify the associated information in an adjacent column. Avoid arranging labeled information (e.g., Patient name: Jones, Thomas Weight: 185 lbs. Height: 74 inches) in what appears to be a paragraph with several chunks of text and numbers.

270. Screen layout. Present critical information in the most prominent location in the most prominent location on the screen.

271. View simplification. Give users the opportunity to temporarily dismiss or hide distracting and/or unnecessary information, but only if it does not contain essential information and controls.

272. Label appearance. Make labels visually subordinate to the related information (i.e., make the information stand out more). For example, presuming a light gray background, make label text medium-dark blue and associated parameter values black.

273. Menus. Display all menu options in a single view, excepting cases in which secondary (i.e., cascading, branching, multi-level) options may be shown or hidden (i.e., collapsed). Place the menu options in a logical order based on function, frequency of use, or (lastly) name (i.e., place in alphabetical order). Ensure that all options logically belong in a given menu so that misplaced option does not disrupt the users' mental model of the menu structure or frustrate a user's search for it in other locations. 
274. Menu selection. Utilize a distinct visual styling to distinguish the currently selected menu option from the unselected menu options.

275. Disabled functions. Visually differentiate functions that are available from those that are unavailable. For example, if certain controls appear "grayed out," no other onscreen elements, and particularly no active functions, should have the same grayed-out appearance or similar appearance.

276. Active links. Present active links using a single, distinct, and exclusive visual style. Avoid using this same visual style for presenting static text.

277. Tabs. Give tabs an appearance that is distinct from adjacent and similar objects (e.g., buttons).

278. Table cell sizing. Avoid making table cells unnecessarily tall or wide such that a large table requires extensive scrolling. In browser applications, limit the degree to which column widths will expand for no purpose, for example. Conversely, ensure that table cells are sufficiently large to display complete entries rather than truncate them.

279. Table columns. Ensure that all table columns are visible when possible. If a column cannot be displayed due to space constraints, provide a conspicuous indication that additional information is available outside the visible area.

280. Table rows. Make table rows visually distinct (e.g., color or shade every other row) to help users keep their place within the table.

281. Table sorting. Enable users to sort tabular data per the contents in particular columns, in ascending or descending order. For example, enable users to sort a table by patient name by clicking on the name column.

282. Browser window size. Do not allow users to shrink windows beyond the point that essential information remains visible.

283. Unit placement. Place units of measures near the associated parameter value. Common placements are to the immediate right or below the values. However, they may also be placed in parentheses to the right or below the parameter title, which itself may ideally be placed above the numerical values. Each of these placements helps to ensure that users will properly associate the values and units of measure. However, make sure to include sufficient space between the unit of measure and the associated parameter value to avoid reading errors due to the visual interference that can arise due to overly close spacing.

284. Overlapping layers. Do not overlap information except in cases where overlapping reinforces a desirable visual effect (e.g., stacked pages/windows); in which case there should be a strong overlap effect (e.g., distinct boundary line, drop shadow). 
285. Window transparency. Avoid making overlapping layers (e.g., windows, pop-ups) transparent to the point that it significantly reduces the legibility of content on the top layer.

286. Window background. When displaying pop-up windows, consider "graying-out" or darkening the background content to make the pop-up window's content more prominent.

287. Distinguishing different screens. Incorporate visual design differences to distinguish between screens that serve significantly different functions. For example, color code patient-related screens with a light blue background and scheduling-related screens with a light green background.

288. Headings and titles. Distinguish screen headings and titles from other on-screen content using color, bolded typefaces, or large font sizes.

289. Differentiation from browser. Visually distinguish the EHR user interface and particularly its associated toolbars from the enclosing browser or operating system controls.

290. Scroll bar visibility. Do not "hide" scroll bars or otherwise require users to discover them.

\section{Visual Design - Graphics (i.e., icons, symbols, charts)}

291. Symbols with conventional meaning. Use symbols (e.g., icons) that have a conventional meaning (e.g., magnifying glass representing a search capability) to convey only those meanings and not others. Otherwise, users might experience negative transfer, leading them to assign the conventional meaning to an icon that the given EHR uses to convey a different meaning.

292. Icon appearance. Size icons so that the salient details that are important to correct interpretation are legible from the maximum, intended viewing distance.

293. Icon composition. Compose individual icons from as many common elements as possible to minimize the number of visual details users must recognize or learn (i.e., limit the visual vocabulary), while also ensuring sufficient distinctiveness among the icons.

294. Icon design. Harmonize the appearance of icons rather than presenting users with variously styled icons. Design variables subject to harmonization include size, aspect (height to width ratio), degree of abstraction, level of detail, use or color, line style, and 2D versus 3D appearance. Generally, representative (i.e., realistic) icons are superior to abstract ones because users are more likely to assign correct meanings to representational ones, while they might have to guess at the meaning of abstract ones other than those already in common use (e.g., red octagon meaning "stop"). 
295. Icon dissimilarity. Make icons sufficiently dissimilar looking so that one will not be confused with another. Significant differences help users tell icons apart (i.e., disambiguate).

296. Icon size. Make icons sufficiently large to ensure that their content is legible and understandable.

297. Icon style. Harmonize the appearance of functionally related icons (e.g., open window/close window, adult patient/pediatric patient). Visually harmonious icons may have the same size, degree of simplification (i.e., abstraction), level of detail, line width, and color saturation, for example.

298. Complementary text labels. Complement icons with text labels or tooltips that clarify the icon's meaning.

299. Branding. Keep vendor brand content from having a negative effect on the EHR's safety, effectiveness and usability. For example, while a login window might be heavily branded, including the vendor's logo; other pages might only employ a colored frame linked to the vendor brand and eliminate other branding details (e.g., logos, tag lines) in favor of leaving more space for useful content.

300. Data visualization. Present data in graphical formats (e.g., parameter value vs. time) when it will communicate essential information (e.g., trends, overall conditions) faster than a table of numbers, for example.

301. Graph axes. Display a graph's axes labels, including units of measure and graduation marks as appropriate, at all times.

302. Graph axis range. If graph axes ranges are set automatically by the EHR, ensure that the ranges are set to realistic values for the displayed parameter. For example, an axis on a graph of body mass index (BMI) values should range from 5 (the minimum likely value) to 60 (extremely obese) containing normal range hard axis lines at BMI 15 and BMI 30.

303. Graph color coding. When presenting multiple trends on a single graph, avoid relying only on color coding to distinguish between the different trends. Utilize distinct symbols as data points (e.g., square, circle, triangle) to differentiate between the different trends.

304. Graph information. Augment graphs with helpful information including meaningful titles, axes labels, symbol keys, and other supplemental information that will facilitate information acquisition and decision-making.

305. Graph options. Enable users to graph selected parameters when this capability is necessary to support clinical analysis and decisions. 
306. Graph size. Ensure that graphs are sufficiently large, or can be viewed at different resolutions for example, so that users can extract the necessary data, such as the value of a vital sign at a certain point in time. Do not reduce the size of graphs to the point that they become unreadable, except when the graph will serve simply as a thumbnail suitable for enlargement to a readable size.

307. Legend. Where appropriate, provide a legend (aka key) that defines the meaning of icons and other symbols used in the EHR.

308. Normal range(s) on graphs. Indicate the normal range for the data shown on graphs.

309. Precise values of plotted data. Give users the means to determine the precise value of plotted data points, such as hovering over a particular data point to produce a popup presenting the value.

310. Time frames of graphs. Display graphs at a default scale that is optimal for data analysis. For example, an adult patient's weight might be best displayed on a graph showing weight change over many years whereas a child's weight graph might be best displayed on a graph showing weight change over many weeks or even days.

311. Toggle ambiguity. Take steps to avoid toggle ambiguities; cases in which there is a choice of two settings (i.e., states) and it is difficult to determine which one is selected based on the appearance of the settings. Ambiguity often arises because users cannot determine if a highlight option is already selected or available for selection. One solution is to add visual detail (e.g., checkmark) to indicate the selected (i.e., currently active) option.

312. Trends. Display (or give users the option to display) trends among appropriate parameters when projections can be helpful. Clearly differentiate actual and projected values.

313. Icon conventions. Use icons in a manner that is consistent with established conventions (i.e., avoid using familiar icons to represent concepts or actions that they do not typically represent). For example, do not use an icon of an envelope to represent a search function, because users will likely associate the envelope icon with a messaging-related functionality.

314. Charting by exception. Enable users to "chart by exception," whereby they can information update a data set by recording only the values that have changed since the last update. This approach should reduce clinicians' workload, reduce the chance of data entry errors, and enable users to focus on correctly entering fewer values.

315. Consistent scales. In cases where users might be comparing trends across multiple charts (e.g., comparing multiple children's growth curves), present the different charts using a consistent scale and axes to facilitate an accurate comparison between the graphs. 


\section{Visual Design - Typography}

316. Information distinctiveness. Present specific types (i.e., categories) of information in distinctive forms so users more readily recognize their purposes. For example, present interaction instructions and clinical information in different forms. Possible ways to differentiate text, for example, include font (i.e., typeface), size, color, background, and paragraph width.

317. Text and number sizes. Ensure that text and numbers are legible from the expected viewing distance (e.g., 18 inches away from a display). They should subtend a visual angle of at least 16 minutes of arc and optimally 20-24 minutes of arc from the maximum expected viewing distance to be accommodated. Note that some EHR content might be viewed at an arm's reach from the computer monitor while other content might be viewed from the opposite side of a hospital bed.

318. Text formatting. Utilize text formatting (e.g., bold, large, or colorful text) to highlight critical details and important information.

319. Text style. Limit variations in text style (e.g., font, boldness, italics, underlining) to a minimum. Generally, use a sans-serif typeface (e.g., Arial, Futura, Helvetica, Verdana) to avoid legibility issues that might arise with the use of typefaces with embellishments, which usually take the form of short lines extending from the ends of the lines that form the letters. Be sure that the text style ensures clear distinction between all letters and numbers. For example, there should be distinct difference in the appearance of the numbers " 1 " and "7."

320. Capitalization. Indicate how text entries should be capitalized when capitalization is important. Otherwise, do not require users to use a specific type of capitalization when entering data. Also, limit the use of capitalization to highlighting information, for example, because reading time can increase when the words in sentences are fully capitalized.

321. Decimal point. Format decimal points to ensure they are highly visible. Formatting options include oversizing the decimal (e.g., make it many times larger than the dot on a similarly sized letter "i")

322. TALLman letters. Where helpful at differentiating similar looking drug names, Use TALLman letter that highlight in capitals letterforms the distinctive letters within the name (e.g., chlorproPAMIDE, chlorproMAZINE).

323. Line spacing. Ensure that the descenders (i.e., the bottom parts of lowercase letters such as " $g$ ") from one line of text are separated from top of the letter in underlying text by enough space to ensure text legibility.

324. Anti-aliasing. Ensure that all text has a "crisp" anti-aliased setting to prevent the appearance of blurred text that users might struggle to read. 
325. Bitmapped fonts. Avoid using bitmapped fonts, which tend to have a pixelated and less legible appearance than vector-based fonts.

326. Data labels. Format data (either static or in input fields) such that it is more prominent than the accompanying labels. For example, when displaying a patient's blood pressure, utilize a larger and/or bolder font for the current blood pressure value (e.g., "120/80 $\mathrm{mmHg}$ ") than for the accompanying label ("Blood pressure:").

327. Decimal point alignment. Display columnar numerical data in decimal point alignment (i.e., with decimal points placed in the same vertical line).

328. Horizontal spacing. Incorporate sufficient horizontal space around displayed text so that individual words do not span multiple lines (i.e., hyphenated across multiple lines).

\section{Workflow}

329. Workflow. Organize EHR content so that it facilitates a safe, effective, and efficient workflows.

330. Consistency with clinical workflow. Explore opportunities to maximize consistency between the EHR's workflow and established clinical workflows with which users are likely to be familiar.

331. Role-related workflows. Present workflows that match the user's role (e.g., nurse, physician, administrative user) by organizing content and functions to match the particular user type's typical tasks. For example, present nurses with common nursingrelated EHR tasks (e.g., entering patient vital signs) immediately on the EHR's home screen.

332. Flexibility. Enable users to perform certain tasks in different manners based on their preference and/or experience.

333. Prerequisite actions. Notify the user about prerequisite actions before allowing them to start a task.

334. Task progression. Provide users with the next logical content when they are progressing through a multi-step procedure rather than requiring them to return to a starting point and then navigating to the next logical content. 
335. Procedural steps. Divide lengthy procedures into discrete "chunks" that follow a natural workflow. Avoid creating overly minor steps (e.g., separate steps to enter birth date and then driver's license) when a single step could include multiple sub-steps (e.g., entering data into several fields on a single window). Indicate progress on a given procedure (e.g., checkmark the completed steps in a summary of all required steps displayed adjacent to a current task window or in a status bar).

Figure 3-25: An example of procedural steps that give the user an indication of their progress through the application.

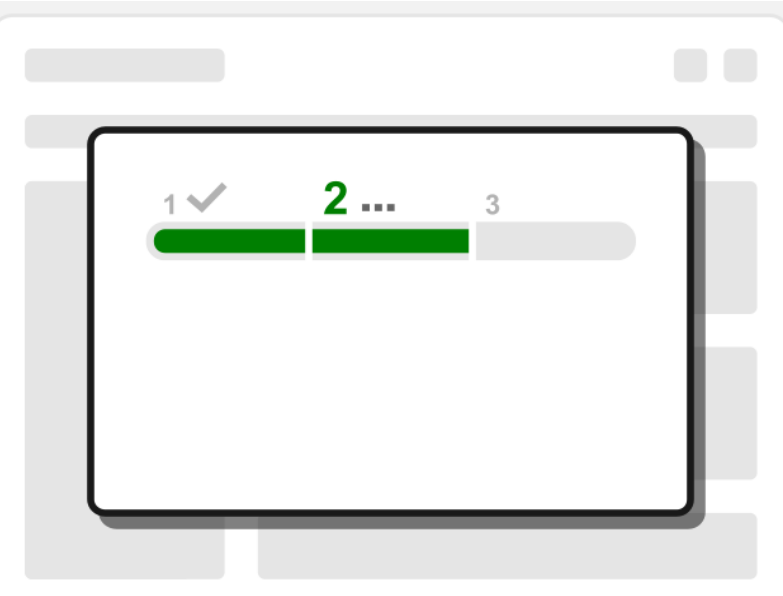

336. Procedural step numbering. Number procedural steps that should be performed in a particular order. Numbering has the added benefit of helping users keep their place in a procedure and differentiating procedural information from other types of information.

337. Accelerators. Provide experienced users with the means to accelerate frequent tasks, just as long as the accelerators do not complicate tasks for inexperienced users. In other words give experienced users shortcuts. Common accelerators are function keys and top-level links to frequently selected options (e.g., frequently ordered laboratory tests).

338. Reminders. Provide helpful reminders to perform task that might be overlooked, such as providing patients with educational materials.

339. Multi-tasking. Enable multi-tasking (e.g., opening two patient charts at a time) where it is necessary to complement a safe and effective workflow.

340. Modal windows. Require users to complete tasks presented within a window, such as confirming an inputted value, before they can interact with other windows.

341. Multi-user communication. Enable communications (e.g., chatting, email messages) with other staff members, or groups of staff members, who use the EHR, and possibly those using other systems, as necessary to facilitate effective medical practice. 
342. Printing. Enable users to readily print selected information from various parts of the EHR.

343. Use of external systems. Minimize the need for users to access external systems and tools (e.g., web sites, other applications) in the course of EHR use. Seek opportunities to incorporate external functionality into the EHR to simplify the user's workflow. 


\section{Appendix A: Additional details about project activities}

\subsection{Usability testing of EHRs}

In the course of this data collection effort, the research team conducted comprehensive usability tests of five EHRs.

Researchers adopted a formative evaluation test approach given that it would enable a rich flow of information about the test participants' experiences using the selected EHRs while they performed the same basic tasks. The testing goal was not to validate the EHRs' user interfaces, but rather to identify their interactive strengths and shortcomings, which could then support the development of user interface guidance to optimize an EHR's user interface.

In 2012, five EHR developers volunteered their EHRs for usability testing in response to a NIST announcement that appeared in the Federal Register on February 14, 2012 (Vol. 77, No. 30). Coincidentally, each of these EHRs was intended primarily for use in outpatient medical practices, such as those used in outpatient clinics and physicians' offices.

The EHR developers provided access to realistically configured EHRs, supported production of a 15-35 minute training video, and provided technical support. They had no input into test planning, testing, or data analysis. Moreover, none of the developers received data resulting from the test of their particular EHR.

Each usability test session lasted approximately two hours; enabling participants to perform up to of 15 separate, predefined tasks (see listing in section 6.1 - Usability testing tasks). There were a total of 15 test sessions per EHR, resulting in a total of 63 participants, including the following:

- Providers $(n=31)$

- $\quad$ Nurses $(n=21)$

- $\quad$ Medical assistants $(n=11)$

Several two-person teams, each including a test administrator and a data analyst (i.e., data logger), conducted the five EHR tests. All test sessions took place in usability test facilities that included a test room and adjacent observation room. All test sessions were video recorded. Spreadsheets containing the raw usability test data were delivered to NIST.

Test data included use errors, close calls, difficulties, anecdotal comments about EHR interactions and quantitative ratings of the EHR according to a novel scoring system (see section $4.2-E H R$ usability scoring system). All test data was de-identified to protect the identities of the test participants, EHRs, and test locations. The EHRs were de-identified because the research focus was not on the usability of the five sample EHRs, but rather on collecting evidence of EHR 
user interface features that contribute to an application's use safety and effectiveness, as well as usability by extension.

After documenting the test results in standalone documents, researchers "coded" the test data according to the methods described in section 4.3 - Qualitative analysis methods. This activity produced 777 coded findings that served as an input to the user interface design principles development effort.

\subsection{EHR usability scoring system}

As part of the EHR usability testing effort (see section 4.1 - Usability testing of EHRs), the research team administered an experimental user experience quality scoring exercise that focused on use safety, effectiveness, and usability.

Readers might be familiar with multiple scoring systems that developers have used for years to evaluate the usability of various types of software applications. Perhaps the most widely used system is the System Usability Scale (SUS) ${ }^{11}$, which calls upon respondents to agree or disagree to various degrees with the following statements:

- I think that I would like to use this system frequently.

- I found the system unnecessarily complex.

- I thought the system was easy to use.

- I think that I would need the support of a technical person to be able to use this system.

- I found the various functions in this system were well integrated.

- I thought there was too much inconsistency in this system.

- I would imagine that most people would learn to use this system very quickly.

- I found the system very cumbersome to use.

- I felt very confident using the system.

- I needed to learn a lot of things before I could get going with this system.

SUS and other scoring systems inspired the development of an EHR-specific scoring system.

Initially, researchers wrote 30 statements (10 safety-related), some derived in part from other scoring system statements, which could be used to assess an EHR's interactive quality. Next, they drew upon their professional judgment to reduce the list to an arguably more manageable 15 statements (see section 4.2.1 - EHRUS usability scoring system development).

${ }^{11}$ See http://www.usability.gov/how-to-and-tools/methods/system-usability-scale.html 
Working within IRB-related restrictions placed on the research, the research team further narrowed the list down to the following 11 statements that constituted just a sample of the kinds of statements that might be proven to be effective indicators of an EHR's usability:

- The EHR uses familiar terms, symbols, and units.

- The EHR makes important information stand out.

- The EHR's workflows match my expectations.

- $\quad$ The EHR makes it easy to detect and correct mistakes.

- The EHR gives me a comprehensive view of a patient's health.

- The EHR will prevent harmful mistakes.

- The EHR makes it easy to share information with other healthcare professionals.

- I can use the EHR effectively while working under pressure.

- $\quad$ The EHR is easy to learn to use.

- I am confident that I can use the EHR to perform tasks correctly.

These statements were not yet evaluated in terms of their construct validity and inter-rater reliability. Rather, they were considered suitable for use in the experimental exercise to produce a general sense for the value of an EHR-specific scoring system. In view of these limits, the researchers administered the exercise to the $62 \mathrm{EHR}$ usability test participants. ${ }^{12}$

Each usability test participant interacted with one of the five evaluated EHRs, referred heretofore as EHRs A, B, C, D, and E. The 5-point rating scale allowed the following levels of agreement with each of the statements:

- $1=$ strongly disagree

- 2 = disagree

- $3=$ neither agree nor disagree

- 4 = agree

- 5 = strong agree

Table 4-1 presents the resulting data.

\footnotetext{
${ }^{12}$ One of the 63 usability test participants did not complete the survey due to test administrator oversight.
} 
Table 4-1 Results of EHR usability scoring exercise.

\begin{tabular}{|c|c|c|c|c|}
\hline EHR & Mean Rating & Std. Dev. & $\begin{array}{l}\text { CV (Coefficient } \\
\text { of Variance }\end{array}$ & $\begin{array}{l}n \text { (number of } \\
\text { participants) }\end{array}$ \\
\hline A & 3.2 & 1.15 & 0.36 & 15 \\
\hline B & 2.5 & 0.94 & 0.38 & 15 \\
\hline C & 2.9 & 1.02 & 0.36 & 15 \\
\hline D & 2.8 & 1.06 & 0.38 & 15 \\
\hline $\mathbf{E}$ & 3.3 & 1.16 & 0.35 & 15 \\
\hline All & 2.9 & 1.07 & 0.36 & 75 \\
\hline
\end{tabular}

The data should be analyzed for trends, and the repeatability of the findings for trend analysis. Accordingly, it is not certain that EHR E would emerge as the most usable EHR if the researchers asked 62 additional participants to perform the scoring exercise, or if there has been a central tendency among raters who would give a mid-range rating even to a demonstrably superior EHR.

Notably, the coefficient of variation (ratio of the mean to the standard deviation) of the limited data set ranges from 0.35 to 0.38 , which suggests modestly good but uncertain inter-rater reliability.

Further research will be required to determine the construct validity and inter-rater reliability of an EHR usability scoring system. Such research will need to compare the results of scoring exercises to other, credible measures of EHR usability (e.g., task times, error rates, expert judgments), as well as other, established usability scoring systems (e.g., SUS). Researchers might be well served to utilize the full set of 30 statements provided in section 4.2.1 - EHRUS usability scoring system development and determine which statements are the best indicators of an EHR's true interactive quality. Further analysis might include a factor analysis of the larger set of 30 statements to reveal clusters of related characteristics, as a springboard for selecting an abbreviated set of statements.

\subsubsection{EHRUS usability scoring system development}

\subsubsection{EHRUS Version 1}

1. The EHR gives me a comprehensive view of a patient's health.

2. The EHR makes it easy to find information.

3. I am confident that I can use the EHR to perform tasks correctly.

4. The EHR's content is poorly organized.

5. The EHR will prevent time-wasting mistakes. 
6. The EHR makes it easy to share information with other healthcare professionals.

7. The EHR will help me deliver higher-quality patient care.

8. I will not remember how to perform infrequent tasks with this EHR.

9. The EHR makes it easy to detect and correct mistakes.

10. The EHR's workflows match my expectations.

11. I will eventually master the EHR's advanced features.

12. The EHR indicates progress on multi-step tasks.

13. The EHR makes it clear what to do next.

14. The EHR's various parts are not well integrated.

15. The EHR provides the information necessary to make good decisions.

16. Using this EHR will increase my workload.

17. The EHR makes important information stand out.

18. The EHR is easy to learn to use.

19. The EHR will prevent harmful mistakes.

20. It takes too long to enter data into the EHR.

21. It will be easy to help co-workers learn to use the EHR.

22. The EHR does not follow consistent rules on how to use it.

23. The EHR makes it clear why it is recommending certain actions.

24. Using the EHR will not improve patient safety.

25. The EHR uses familiar terms, symbols, and units.

26. The EHR includes only necessary and useful features.

27. I can use the EHR effectively while working under pressure.

28. The EHR requires me to enter the same information too many times.

29. The EHR makes it easy to share information.

30. The EHR is poorly designed for people who do my job. 
The following statements (a subset of above list) are particularly use-safety related:

31. The EHR gives me a comprehensive view of a patient's health.

32. The EHR makes it easy to find information.

33. I am confident that I can use the EHR to perform tasks correctly.

34. The EHR makes it easy to share information with other healthcare professionals.

35. The EHR will help me deliver higher-quality patient care.

36. The EHR makes it easy to detect and correct mistakes.

37. The EHR provides the information necessary to make good decisions.

38. The EHR will prevent harmful mistakes.

39. The EHR makes it clear why it is recommending certain actions.

40. Using the EHR will not improve patient safety.

41. The EHR uses familiar terms, symbols, and units.

\subsubsection{EHRUS Version 2}

42. The EHR uses familiar terms, symbols, and units.

43. The EHR makes it easy to share information.

44. The EHR makes important information stand out.

45. The EHR indicates progress on multi-step tasks.

46. The EHR's workflows match my expectations.

47. The EHR makes it easy to detect and correct mistakes.

48. The EHR gives me a comprehensive view of a patient's health.

49. The EHR makes it easy to find information.

50. The EHR will prevent harmful mistakes.

51. The EHR makes it easy to share information with other healthcare professionals.

52. I can use the EHR effectively while working under pressure. 
53. The EHR's various features and functions are well integrated.

54. The EHR includes only necessary and useful features.

55. The EHR is easy to learn to use.

56. I am confident that I can use the EHR to perform tasks correctly.

\subsection{Qualitative analysis methods}

Each of the EHR user interface principles presented in this document are linked to one or more project research findings; the product of an intensive qualitative data analysis.

The qualitative analysis approach involved the following steps:

- Determine units of analysis - Analysts reviewed the research data and determined the appropriate unit of analysis for each data source, as follows:

o Web survey - survey respondents' answers to two questions posed in the Webbased survey ("What EHR characteristics make it particularly easy to use?" and "What EHR characteristics make it particularly difficult to use?")

o Site visit interviews - answers to questions posed to clinical and administrative interviewees during site visits

o Site visit observations - field researchers' observation notes that comprised a finding focused on a particular usability issue, safety concern or task step

o Usability testing - use errors, close calls (cases when a user almost commits a use error), difficulties, instances of test administrator assistance, and subjective feedback (i.e., anecdotal usability test participant comments provided during a task or follow-up interviews)

o Expert reviews - a specific finding (i.e., user interface design strength or weakness), accompanied by potential benefits or consequences and potential design improvements

- Determine finding codes - Analysts reviewed the 15 expert review categories (see section 4.4.1 - Expert review categories) and, drawing on professional judgment and an initial review of the findings from the other research activities, expanded the number to 25 to cover a wide range of user interface design topics. During the coding process, four additional categories ("Billing", "Interoperability", "Staff Communication", and "Patient Education/Communication") emerged as necessary to enable sufficiently specific data coding and were added to the list of 25 categories, thereby resulting in a total of 29 categories (see section 4.4.2 - Complete list of categories). 
- Train analysts to ensure consistent coding - A lead researcher trained three analysts to code the research findings to ensure consistency. The researcher described each of the coding categories individually and provided examples of associated findings from various data sources and then selected and coded a set of 30 findings selected at random from the site visit data and asked each of the three analysts to code these findings independently. The researcher then led a discussion during which each analyst reported their codes for each finding, and the researcher provided clarification and led a discussion to resolve discrepancies between raters. Additionally, the lead researcher was available to discuss any questions that arise throughout the entire coding process.

- Code the findings - The lead researcher and three analysts reviewed all of the data in raw or processed form and coded each finding (i.e., unit of analysis, as described above) with one or two categories: a primary code, capturing the main aspect of interest for the finding, and potentially a secondary code, capturing any additional relevant information. Importantly, each data source was coded by two or more individuals to limit potential bias resulting from a particular analyst's perspective on a particular data source.

For example, the following usability test finding was coded as "Clinical Decision Support"(primary code) and "Visual Design" (secondary code):

"One provider suggested displaying abnormal lab results, or those that require follow-up, in red to help users to distinguish between critical and non-critical results"

Ultimately, the analysts coded over 3,400 findings. Some findings were coded as "N/A" if they were not relevant to design guidance, were not technically compelling, or were insufficiently detailed to support a principle.

Table 4-2 lists the number of findings generated from each data source.

Table 4-2: Number of findings generated from each data source.

\begin{tabular}{|c|c|c|}
\hline EHR & Number of findings & $\begin{array}{l}\text { Number of coded } \\
\text { findings leading to } \\
\text { principles }\end{array}$ \\
\hline Usability testing & 806 & 777 \\
\hline $\begin{array}{l}\text { Site visit interviews and } \\
\text { observations }\end{array}$ & 1,389 & 927 \\
\hline Expert reviews & 665 & 665 \\
\hline Web survey & 592 & 350 \\
\hline Total & 3,452 & 2,719 \\
\hline
\end{tabular}


- Ensure coding quality control - The three analysts marked findings about which they were uncertain about the tentatively assigned codes. The lead analyst reviewed and adjusted the codes assigned to these findings, and also performed spot checks on randomly selected findings as an additional coding quality check.

- Synthesize principles - Analysts and two principal researchers reviewed the coded findings by category (e.g., "Alerts") or multiple categories (e.g., "Workflow" and "Efficiency") and formulated principles where they judged there to be sufficient evidence to support them. Ultimately, the analysts wrote over 380 principles, many of which were based on multiple findings. Multiple findings pertaining to the same topic was considered a strong indicator that a principle was warranted.

- Review prinicples - Multiple analysts judged the integrity of the principles, by crosscomparing them to principles from other authoritative sources and their professional expertise. This review led to some principle deletions as well as to editorial adjustments to increase clarity and conciseness. This step reduced the number of principles from 380 to 343.

- $\quad$ Link principles to codes - Analysts produced a spreadsheet that links the 343 EHR user interface design principles to the coded research findings. Analysts reviewed each finding for its potential to support a particular principle, and also reviewed principles to determine which additional findings further supported them.

\subsection{Usability finding categories}

\subsubsection{Expert review categories}

This section lists the 15 categories used to structure and categorize the expert review findings. This list was created based on the collective expert judgment of UL-Wiklund and its collaborators, as well as a review of Nielsen's 10 heuristics for user interface design (Nielsen, 2005) ${ }^{13}$, Shneiderman's 8 Golden Rules of Interface Design (Shneiderman, 1992) ${ }^{14}$, and 14 usability heuristics developed for the medical industry that were later incorporated into the TURF framework (Zhang, 2003; Zhang \& Walji, 2011) ${ }^{15}$.

\section{Content Organization}

Clarification: Pertains both to content on a single screen and the distribution of contents among multiple screens.

\footnotetext{
${ }^{13}$ Nielsen, J. (2005). Ten usability heuristics.

${ }^{14}$ Shneiderman, B. (1992). Designing the user interface: strategies for effective human-computer interaction (Vol. 3). Reading, MA: Addison-Wesley.

${ }^{15}$ Zhang, J., \& Walji, M. F. (2011). TURF: Toward a unified framework of EHR usability. Journal of biomedical informatics, 44(6), 1056-1067. Chicago; Zhang, J., Johnson, T. R., Patel, V. L., Paige, D. L., \& Kubose, T. (2003). Using usability heuristics to evaluate patient safety of medical devices. Journal of biomedical informatics, 36(1), 23-30.
} 
The configuration (physical arrangement, order of presentation) of information (e.g., text, graphics, charts, tables) within a screen and the degree to which it facilitates information acquisition, data entry, and control tasks.

\section{Navigation}

Clarification: Pertains to movement among multiple screens.

The clarity and efficiency of movement among an application's various screens.

\section{Nomenclature}

The use of familiar terms for the intended users.

\section{Visual Design}

The use of graphics, color, typography, layout, and other visual elements to facilitate information acquisition, draw attention to important information, and optimize the aesthetic appeal of onscreen content.

\section{Error Prevention}

Design characteristics that protect users from committing use and/or safety errors.

\section{Error Recovery}

Design characteristics that help users detect and recover from use and/or safety errors, giving users a chance to check their actions and make necessary corrections before the error becomes consequential.

\section{Feedback}

Indications that a user action is required, and/or information that a user action has been accepted/submitted, or otherwise is in progress. Feedback from an EHR may take visual and/or auditory forms.

\section{Status Indication}

Information about system processes and progress (e.g., search is being undertaken, document is being downloaded, print job is being sent to printer), or clinical processes and progress (e.g., order status, pending tasks).

\section{Internal Consistency}

The EHR employs a common set of design rules and behaviors that enable users to apply lessons learned about performing a given task with the EHR to other tasks within the EHR.

\section{External Consistency}


An EHR employs a set of design rules and behaviors that are consistent with EHRs and other EHRs and software products with which a user might be familiar, such as business applications, websites, medical devices, and consumer products.

\section{User Support}

The use of user interface features such as headings, prompts, alerts, tool-tips, reminders, and online help to guide users' interactions with the EHR.

\section{Support for clinical decision making}

The EHR provides information necessary to make proper medical decisions and choices at the right time.

\section{Affordances}

Clarification: Pertains to user interface design characteristics that are intrinsic (e.g., large text that increases legibility, a drop shadow on a button that distinguishes the button from a text field, a button that has both an icon and text label) rather than elements normally regarded to be forms of user support.

Information contained within the design element informs actions that can be undertaken with the element (e.g., "buttons" that afford pushing, accordion chevrons that afford expanding and collapsing).

\section{Customizability}

Opportunities for users to adjust certain EHR characteristics (e.g., available functions, information configurations, degree of prompting) to suit their personal needs and preferences.

\section{Workflow}

Clarification: Pertains to EHR-related tasks in the context of preferred clinical practices.

The efficiency with which users can perform overall EHR-related tasks, which may occur in parallel or in series with interactions involving other equipment and personnel, and the degree to which it matches users' preferred task order and established mental models.

\subsubsection{Complete list of categories}

This appendix lists the 29 categories used to categorize the research findings from all data sources. Note that the categories pertain to either user interface design principles (i.e., "Content Organization") or particular EHR-specific tasks and aspects (i.e., "Patient Identification.").

Emergent categories (i.e., that arose organically from themes discovered during the coding process) are marked with a star.

- Affordances 
- Clinical Decision Support

- Conceptual Model

- Content Organization

- Information density

- Customizability

- Data Entry

- Internal Consistency

- External Consistency

- Efficiency

- Workflow

- Error Prevention, Detection, and Recovery

- Alerts

- Feedback

- Language

- Navigation

- Patient Identification

- Status Indication

- Search

- User Support

- Visual Design

- Typography

- Graphics

- Color

- Metaphor

- Billing*

- Interoperability*

- Patient Education/Communication*

- Staff Communication* 


\section{Appendix B: EHR development best practices}

Research team members conducted an interview with Dr. Raj Ratwani, Scientific Director at MedStar's National Center for Human Factors in Healthcare, regarding recent research the center conducted about EHR vendors' user-centered design (UCD) processes. ${ }^{16}$ Dr. Ratwani and a team of human factors experts interviewed eleven EHR vendors determine current vendor UCD practices and develop an appreciation for the difficulties vendors face incorporating UCD into EHR design and development processes.

Based on the interview with Dr. Ratwani, the research team developed a set of EHR usability engineering best practices regarding the incorporation of UCD into EHR design and development. Each best practice describes its associated benefits, current EHR vendor practices, and recommendations for implementing the practice.

\subsection{Promote usability at the leadership level}

Strong leadership support for usability engineering helps build an organizational culture that values and prioritizes user-centered design activities.

Current practices: Leadership support for UCD varies among EHR vendors, from knowledgeable and supportive leadership who view EHR usability as a market differentiator to generally unsupportive or un-knowledgeable leadership who have an overly limited view of usability engineering (e.g., mistakenly considering post-market feedback solicitation as a complete usability engineering program).

Recommendations: EHR vendor leadership should ensure they are well-informed regarding basic user-centered design principles and their effect on EHR usability, use safety, and commercial success. Additionally, leadership should champion UCD processes and usability both at internal meetings and by allocating sufficient financial and scheduling resources for UCD activity and dedicated human factors expertise, either in-house or via a consulting practice. Finally, human factors and usability engineering staff should inform colleagues about the value of user-centered design, including calculating or estimating the potential return benefits and demonstrating how particular user feedback.

\subsection{Establish and maintain a diverse user base}

A diverse user network enables vendors to obtain rapid feedback throughout the design and development process.

\footnotetext{
${ }^{16}$ Ratwani, R. M., Fairbanks, R. J., Hettinger, A. Z., \& Benda, N. C. (2015). Electronic health record usability: analysis of the user-centered design processes of eleven electronic health record vendors. Journal of the American Medical Informatics Association.
} 
Current practices: Current practices vary widely. Some vendors run usability tests at their annual conference, which is likely to be attended by many of their customers, while others have a network of users they can contact throughout the year.

Recommendations: Engage the user base to participate in regular user research activities. Consider identifying a core group of users representing diverse demographic characteristics and clinical specialties. This core clinician network will enable rapid recruitment of usability test participants and the collection of user feedback throughout the design and development process. Vendors can increase clinicians' motivation to participate in such a network by emphasizing their impact on the product design and showing participants examples of how their feedback led to design changes.

\subsection{Conduct a variety of user-centered design activities}

Conducting a variety of user-centered design activities helps developers form a multifaceted understanding of user interactions with the product. Plan user-centered design activities to provide relevant and appropriate input during particular phases of the design and development process.

Current practices: Vendors with established UCD procedures tend to conduct a mix of activities, with the most common ones being development of use cases and user profiles or personas to guide the development process and the conduct of formative and/or summative usability tests. Some developers also conduct field observations in the initial design phase. Vendors without an established UCD program tend to conduct a limited subset of UCD activities, typically at only one or two points throughout the EHR development process.

Recommendations: Plan to conduct a range of UCD activities to provide appropriate input to the design and development process. Incorporate activities such as focus groups, card sorting, use case scenario development, interviews, and observations early in the process and transition to formative and finally summative usability testing later in the process.

\subsection{Conduct formative usability tests early and often}

Conducting multiple formative tests in the early stages of the design process, and using test findings to inform product development, facilitates safe and usable EHR design.

Current practices: Some developers skip formative testing based on the false premise that it is an optional step in the UCD process. As a result, developers who skip formative testing are more likely to encounter significant usability and use safety related shortcomings during summative usability testing.

Recommendations: Allocate resources to formative usability testing throughout the product development lifecycle, focusing on phases in which study findings can still inform the EHR design and design changes are less costly (i.e., relative to later-stage testing). 


\subsection{Invest in professional usability expertise and encourage frequent interaction between usability experts and development teams}

A collaborative, open dialogue between the development team and dedicated usability experts promotes the integration of user-centered design into the development process.

Current practices: Vendors with dedicated UCD teams typically follow one of two models. In one model, individual UCD team member work within a particular development team. In the other model, the UCD team serves in an advisory position, training developers and engaging with the development team at key junctures during the development process. Both models have proven to be effective.

Recommendations: Hire dedicated in-house usability professionals or maintains an ongoing relationship with a usability consulting organization. Encourage an ongoing dialogue between usability experts and the development team, within a team-based or advisory framework (see above).

\subsection{Integrate user-centered design into the development process}

Plan for usability testing and improvements from start of the development process to ensure time to address use-safety and usability issues.

Current practices: Vendor organizations typically prioritize a timely, on-schedule product launch. Organizations with well-developed UCD incorporate and plan for usability testing and design improvements in their development timelines.

Recommendations: Put a rigorous UCD process in place from the beginning of the development process that spans the entire product life-cycle, including post-market introduction. Human factors professionals should integrate themselves into the regular software development process without slowing down the development time line. Usability professionals should clearly communicate desired design modifications, explaining the benefits of implementation (vs. costs of nonimplementation) and prioritizing those design modifications that are likely to have the biggest impact in terms of usability and use-safety.

\subsection{Infuse design and evaluation with clinical expertise}

Considering typical and optimal user workflows and clinician's mental models of frequent, urgent and critical tasks will increase product efficiency, usability, and user satisfaction.

Current practices: Some vendors work with clinical consultants; others hire staff with a clinical background. Some vendors invest several hundred hours observing and interviewing clinicians on-site in lieu of the advisory or in-house model. 
Recommendations: Develop user profiles and use cases to better understand representative users. Investigate users' typical workflow and develop an understanding of the cognitive requirements of particular tasks (i.e., through cognitive task analysis), users' mental model of particular tasks, and the nature of user collaboration and interaction. Finally, obtain feedback from trusted clinicians regarding the development of appropriate and technically correct formative and summative test tasks and scenarios.

\subsection{Develop a use-related risk management process}

\section{A use-related risk management process details and analyzes use-related risks, ensuring that the product does not enable users to commit a safety-related use error or that the risk is mitigated to the greatest possible extent.}

Current practices: Few vendors have dedicated patient safety or risk groups that work closely with the usability and development teams.

Recommendations: Incorporate risk analysis into the development cycle. Convene a dedicated multidisciplinary team (e.g., software developers, clinicians, etc.) to determine actions users can take within the EHR that might introduce patient harm. After producing an exhaustive list of such actions, assess each action's likelihood of occurrence, related clinical harm(s), severity of harm, and mitigations currently in place to address said risk. Then, determine additional steps developers can take to reduce or eliminate the risk. After implementing the newly introduced mitigation, again assess the likelihood of occurrence and severity of harm based on the new mitigation. 


\section{Appendix C: Sample documents}

\subsection{Usability testing tasks}

The research team selected tasks that would be realistic and representative of the kinds of activities a user might perform with this EHR, tailored to each of the three user groups. Tasks were developed collaboratively among members of the research team with human factors and clinical expertise. Tasks represent both common and safety-critical EHR functions.

To increase task realism, tasks were integrated into scenarios, which were 1-3 short paragraphs describing a particular patient and clinical need. These scenarios also served to provide clinical context for the tasks.

Table 6-1 lists tasks available to be performed by medical assistant participants.

Table 6-1: Medical assistant tasks.

\section{Scenario Task}

1

1 - Reschedule appointment

2 - Update patient address

2

3 - Find appointment request

4 - Schedule follow-up appointment

5 - Modify location of x-ray order

36 - Print chest x-ray order

7 - Document location change

4

8 - Fax CT scan order

9 - Document order fax

5

10 - Create new patient record

Table 6-2 lists tasks available to be performed by nurse participants.

Table 6-2: Nurse Tasks.

\section{Scenario Task}

$1 \quad 1$ - Enter vital signs 


\begin{tabular}{|l|l|}
\hline & $\mathbf{2}$ - Order rapid flu test \\
\hline $\mathbf{2}$ & $\mathbf{3}$ - Schedule follow-up appointment \\
\hline $\mathbf{3}$ & $\mathbf{5}$ - - -ocate cholesterol lab result \\
\hline $\mathbf{6}$ & $\mathbf{6}$ - Document patient education \\
\hline $\mathbf{7}$ & $\mathbf{8}$ - Docate current orders \\
\hline $\mathbf{5}$ & $\mathbf{1 0}$ - Fax chest x-ray order \\
\hline $\mathbf{1 1}$ - Cancel hematocrit order \\
\hline $\mathbf{6}$ & $\mathbf{1 3}$ - Reschiew growth chart \\
\hline $\mathbf{7}$ & $\mathbf{1 4}$ - Document - Reconcile medications \\
\hline
\end{tabular}

Table 6-3 lists tasks available to be performed by provider participants

Table 6-3: Provider tasks.

\section{Scenario Task}

1 - Review patient information

1

2 - Order referral

3 - Order tests

4 - Check for and place order

5 - Order vaccine

6 - Cancel referral

3

7 - Modify location of bone density scan

8 - Place multiple orders

9 - Order abdominal x-ray 
10 - Schedule follow-up appointment

11 - Add diagnosis

12 - Reconcile medications

5

13 - Adjust dose

14 - Prescribe medications

15 - Print prescriptions

\subsection{Sample cognitive task analysis}

\subsubsection{Introduction}

Developers can develop a deeper understanding of user interactions with an EHR by performing a cognitive task analysis of user tasks, focusing on those ensuring use safety and effectiveness. Cognitive task analysis might sound complex since the term "cognitive" suggests a need for extensive training in psychology or cognitive science. However, a cognitive tasks analysis can be a reasonably straightforward and practical process

Applied Cognitive Task Analysis (ACTA) is a streamlined cognitive task analysis methodology that focuses on efficiently identifying what users and need to know to accomplish a task and, accordingly, the factors developers must take into account to design effective systems. ${ }^{17}$ One form of ACTA, a cognitive demands table, calls upon analysts to determine what is mentally difficult about a task, why it is difficult, what errors the difficulty might induce, and coping strategies that help individuals overcome the difficulty. Note that the focus is on difficulties in this case, but a cognitive task analysis can be approached in a more neutral manner, identifying aspects of various tasks that are easy, neither easy or difficult, or difficult.

This appendix presents the results of a cursory cognitive analysis (pertaining to a hypothetical EHR) that of five difficult and common EHR tasks. It focuses on the following five tasks, which are a small subset of the dozens of tasks that people might perform using a given EHR:

- Prescribe medication

- Order procedure

- Manage problem list

- Enter vital signs

- Schedule appointment

\footnotetext{
17 Militello, L. G., \& Hutton, R. J. (1998). Applied Cognitive Task Analysis (ACTA): A practitioner's toolkit for understanding cognitive task demands. Ergonomics, 41(11), 1618-1641.
} 
The results of such an analysis, or analyses of a similar nature, can be a rich source of inspiration for user interface designers who seek to streamline tasks by making cognitive tasks easier to perform correctly. Possible user interface design solutions linked to the sample cognitive task analysis findings are presented below as a demonstration of the potential value of such an analysis.

Table 6-4: Cognitive task analysis insights and resulting design solutions.

\begin{tabular}{|c|c|c|}
\hline Task & Challenge & Potential UI Design Solutions \\
\hline $\begin{array}{l}\text { Open patient } \\
\text { chart }\end{array}$ & $\begin{array}{l}\text { Clinicians might open the wrong } \\
\text { patient's chart because they have } \\
\text { multiple patient charts open at once }\end{array}$ & $\begin{array}{l}\text { Persistently and conspicuously } \\
\text { present key patient information (e.g., } \\
\text { name, date of birth, photograph) in } \\
\text { the patient chart and accompanying } \\
\text { controls (e.g., tab used to select the } \\
\text { patient chart). }\end{array}$ \\
\hline $\begin{array}{l}\text { Prescribe } \\
\text { medication }\end{array}$ & $\begin{array}{l}\text { Clinicians might confuse medications } \\
\text { with similar spellings or purposes }\end{array}$ & $\begin{array}{l}\text { Present medication name using a } \\
\text { large font (e.g., greater than } 18 \text { point } \\
\text { font size). } \\
\text { Utilize TALLman lettering to highlight } \\
\text { spelling differences between } \\
\text { similarly named medications. } \\
\text { Require users to confirm they } \\
\text { selected the correct medication. }\end{array}$ \\
\hline $\begin{array}{l}\text { Prescribe } \\
\text { medication }\end{array}$ & $\begin{array}{l}\text { When ordering prescriptions, } \\
\text { clinicians must recall and consider } \\
\text { patient diagnoses, which might not be } \\
\text { presented alongside medication } \\
\text { ordering module }\end{array}$ & $\begin{array}{l}\text { Present relevant patient details (e.g., } \\
\text { allergies, currently prescribed } \\
\text { medications) in a persistent manner, } \\
\text { such that they are visible on all } \\
\text { ordering screens. } \\
\text { Utilize algorithms to automatically } \\
\text { detect any conflicts between patient } \\
\text { and medication characteristics. If a } \\
\text { potential conflict is detected, present } \\
\text { an alert message to the user. }\end{array}$ \\
\hline $\begin{array}{l}\text { Prescribe } \\
\text { medication }\end{array}$ & $\begin{array}{l}\text { Some EHRs present an extensive list } \\
\text { of potential interactions but do not } \\
\text { indicate severity, thereby requiring } \\
\text { clinicians to prioritize among contra- } \\
\text { indications }\end{array}$ & $\begin{array}{l}\text { Utilize visual design attributes (e.g., } \\
\text { ordering, color-coding, flashing) to } \\
\text { indicate the relative priority of } \\
\text { displayed interactions and contra- } \\
\text { indications. }\end{array}$ \\
\hline $\begin{array}{l}\text { Search for } \\
\text { procedure }\end{array}$ & $\begin{array}{l}\text { Clinicians might not distinguish } \\
\text { between inclusive (i.e., result must } \\
\text { include all search terms) and } \\
\text { exclusive search, thereby potentially } \\
\text { over-specifying the search term such } \\
\text { that the desired procedure does not } \\
\text { appear in the search results }\end{array}$ & $\begin{array}{l}\text { Present a prompt that explains the } \\
\text { syntax used for inclusive vs. } \\
\text { exclusive searches. } \\
\text { When presenting search results, } \\
\text { provide a prompt explaining the } \\
\text { search method used based on the } \\
\text { user's search string. }\end{array}$ \\
\hline
\end{tabular}




\begin{tabular}{|c|c|c|}
\hline Task & Challenge & Potential UI Design Solutions \\
\hline $\begin{array}{l}\text { Add diagnosis } \\
\text { code (s) }\end{array}$ & $\begin{array}{l}\text { Clinicians might not know which ICD } \\
\text { codes will be accepted by the } \\
\text { patient's insurance }\end{array}$ & $\begin{array}{l}\text { Enable users to select diagnosis } \\
\text { codes from a list of available codes. } \\
\text { Enable users to search for a code by } \\
\text { entering associated terms. }\end{array}$ \\
\hline $\begin{array}{l}\text { Audit problem } \\
\text { list }\end{array}$ & $\begin{array}{l}\text { Multiple clinicians can edit a patient's } \\
\text { problem list, potentially leading to } \\
\text { duplicate entries. Clinicians might } \\
\text { disagree regarding the contents of the } \\
\text { patient's active problem list }\end{array}$ & $\begin{array}{l}\text { Use a software algorithm to } \\
\text { automatically identify duplicate } \\
\text { entries and inform the user of their } \\
\text { presence. } \\
\text { Indicate when a patient chart is } \\
\text { being edited simultaneously by } \\
\text { multiple users. Also, indicate which } \\
\text { edits are being made by other users. }\end{array}$ \\
\hline $\begin{array}{l}\text { Audit problem } \\
\text { list }\end{array}$ & $\begin{array}{l}\text { Clinicians might delete rather than de- } \\
\text { activate an entry, thereby removing } \\
\text { important information from the } \\
\text { patient's medical history }\end{array}$ & $\begin{array}{l}\text { Do not enable users to permanently } \\
\text { delete information from a patient } \\
\text { profile (e.g., only enable users to de- } \\
\text { activate information). } \\
\text { Provide a clear indication or warning } \\
\text { informing the user that deleted } \\
\text { information cannot be recovered. } \\
\text { Require users to actively confirm that } \\
\text { they intend to delete the patient } \\
\text { profile (e.g., by entering their EHR } \\
\text { account password to confirm their } \\
\text { intention to delete the profile). }\end{array}$ \\
\hline $\begin{array}{l}\text { Enter vital } \\
\text { signs with } \\
\text { units }\end{array}$ & $\begin{array}{l}\text { Clinicians might measure patient's } \\
\text { vital signs in an order that does not } \\
\text { match the vital sign data fields' order, } \\
\text { thereby leading to data entry errors }\end{array}$ & $\begin{array}{l}\text { Include prominent, conspicuous } \\
\text { labels for each vital sign, to minimize } \\
\text { potential that users will overlook the } \\
\text { label. } \\
\text { Provide alerts regarding abnormal } \\
\text { (or impossible) vital sign ranges or } \\
\text { potential data entry errors and, if } \\
\text { necessary, modify vital sign fields. } \\
\text { Enable hospital administrators to } \\
\text { modify the order of vital sign fields so } \\
\text { that it matches the hospital's } \\
\text { established order forms. }\end{array}$ \\
\hline
\end{tabular}




\begin{tabular}{|l|l|l|}
\hline Task & Challenge & Potential UI Design Solutions \\
\hline $\begin{array}{l}\text { Enter vital } \\
\text { signs with } \\
\text { units }\end{array}$ & $\begin{array}{l}\text { Several vital signs (e.g., height, } \\
\text { temperature) may be recorded in } \\
\text { either English or metric units. } \\
\text { Clinicians might select incorrect units } \\
\text { or neglect to modify default units, }\end{array}$ & $\begin{array}{l}\text { Provide a conspicuous indication of } \\
\text { the currently selected unit. } \\
\text { Automatically calculate and display } \\
\text { the vital sign in its secondary unit } \\
\text { next to the primary unit. } \\
\text { Provide alerts regarding abnormal } \\
\text { (or impossible) vital sign ranges or } \\
\text { potential data entry errors and } \\
\text { modify vital sign fields if necessary }\end{array}$ \\
\hline $\begin{array}{l}\text { Enter vital } \\
\text { signs with } \\
\text { units }\end{array}$ & $\begin{array}{l}\text { Clinicians might enter vital sign } \\
\text { measurements incorrectly (e.g., } \\
\text { misplacing a decimal point, omitting } \\
\text { digits, entering one vital sign in } \\
\text { another vital sign's data field) }\end{array}$ & $\begin{array}{l}\text { Provide alerts regarding abnormal } \\
\text { (or impossible) vital sign ranges or } \\
\text { potential data entry errors and } \\
\text { modify vital sign fields if necessary }\end{array}$ \\
\hline
\end{tabular}




\subsubsection{Prescribe medication}

\begin{tabular}{|c|c|c|c|c|}
\hline Task & Challenge & Performance shaping factors & Potential use errors & Risk reduction strategies \\
\hline $\begin{array}{l}\text { Open patient } \\
\text { chart }\end{array}$ & $\begin{array}{l}\text { Select correct } \\
\text { patient }\end{array}$ & $\begin{array}{l}\text { - Clinicians see many patients (perhaps } \\
\geq 10 \text { ) in a single day } \\
\text { Clinicians might wish to keep multiple } \\
\text { patient charts open at once } \\
\text { - Some patients might have identical or } \\
\text { similar names }\end{array}$ & $\begin{array}{l}\text { - } \quad \text { Open wrong patient chart } \\
\text { - } \quad \text { Confuse one patient's chart for } \\
\text { another }\end{array}$ & $\begin{array}{l}\text { - Confirm patient identity by reviewing at } \\
\text { least two patient identifiers (e.g., } \\
\text { D.O.B., full name, photo) } \\
\text { Constrain the number of patient charts } \\
\text { that might remain open at once }\end{array}$ \\
\hline $\begin{array}{l}\text { Access } \\
\text { prescription } \\
\text { ordering } \\
\text { module }\end{array}$ & $\begin{array}{l}\text { Locate ordering } \\
\text { module }\end{array}$ & $\begin{array}{l}\text { The patient chart's home screen } \\
\text { features relatively high information } \\
\text { density } \\
\text { Exposure to other EHR content } \\
\text { organization schemes can lead to } \\
\text { negative transfer } \\
\text { - Providers might have multiple patient } \\
\text { chart and medication prescription } \\
\text { windows open simultaneously }\end{array}$ & $\begin{array}{l}\text { - Open wrong module due to } \\
\text { confusing nomenclature or content } \\
\text { organization (e.g., open procedure } \\
\text { ordering module rather than } \\
\text { prescription ordering module) } \\
\text { Open wrong patient's prescription } \\
\text { ordering module }\end{array}$ & $\begin{array}{l}\text { Interpret navigation cues by utilizing } \\
\text { icon tooltips } \\
\text { - Verify name on patient ordering } \\
\text { module }\end{array}$ \\
\hline $\begin{array}{l}\text { Search for } \\
\text { medication }\end{array}$ & $\begin{array}{l}\text { Search for correct } \\
\text { medication }\end{array}$ & $\begin{array}{l}\text { Clinicians might confuse medications } \\
\text { with similar spellings or purposes } \\
\text { Medications might be listed under } \\
\text { unexpected names (e.g., generic } \\
\text { instead of brand name) } \\
\text { Medication names can be complex, } \\
\text { have multiple forms, and can change as } \\
\text { similar medications or generic versions } \\
\text { are developed } \\
\text { Search results might be extensive }\end{array}$ & $\begin{array}{l}\text { Type medication name into search } \\
\text { field incorrectly } \\
\text { Select a medication that appears to } \\
\text { be correct in the medication list, but } \\
\text { is incorrect due to: similar } \\
\text { name/spelling, generic alternatives, } \\
\text { wrong dosage, concentration, or } \\
\text { form of medication (e.g., liquid form } \\
\text { instead of tablets) } \\
\text { Inadvertently click on wrong } \\
\text { medication (i.e., selection accuracy) } \\
\text { Add medication to wrong patient's } \\
\text { chart (i.e., if wrong patient chart } \\
\text { selected previously) }\end{array}$ & $\begin{array}{l}\text { Verify medication spelling by referring } \\
\text { to an external website or resource } \\
\text { Confirm medication name and details } \\
\text { are appropriate after selection }\end{array}$ \\
\hline
\end{tabular}




\begin{tabular}{|c|c|c|c|c|}
\hline Task & Challenge & Performance shaping factors & Potential use errors & Risk reduction strategies \\
\hline $\begin{array}{l}\text { Specify } \\
\text { prescription } \\
\text { details }\end{array}$ & $\begin{array}{l}\text { Specify correct } \\
\text { prescription details } \\
\text { (e.g., dosage, } \\
\text { concentration, and } \\
\text { brand) }\end{array}$ & $\begin{array}{l}\text { - Some medications are available in a } \\
\text { relatively high number of dosages, } \\
\text { concentrations, and forms }\end{array}$ & $\begin{array}{l}\text { Inadvertently select incorrect } \\
\text { medication concentration (i.e., } \\
\text { selection accuracy) }\end{array}$ & $\begin{array}{l}\text { - Review all prescription details prior to } \\
\text { finalizing order }\end{array}$ \\
\hline $\begin{array}{l}\text { Review any } \\
\text { contra- } \\
\text { indications } \\
\text { or alerts }\end{array}$ & $\begin{array}{l}\text { Review contra- } \\
\text { indications and } \\
\text { alerts }\end{array}$ & $\begin{array}{l}\text { Patients might have many medications } \\
\text { on their list, such that prescribing a } \\
\text { particular medication might require } \\
\text { modifying the mediation list overall } \\
\text { Clinicians must recall and consider } \\
\text { patient diagnoses and allergies, which } \\
\text { might not be presented alongside } \\
\text { medication ordering module } \\
\text { Some EHRs present an extensive list of } \\
\text { potential interactions but do not indicate } \\
\text { severity, thereby requiring clinicians to } \\
\text { prioritize among contra-indications }\end{array}$ & $\begin{array}{l}\text { Ignore critical drug interaction alerts } \\
\text { due to alert fatigue and rapid clicking } \\
\text { pace } \\
\text { - Order wrong medication (i.e., a non- } \\
\text { effective treatment that is not } \\
\text { indicated for the patient's disorder) } \\
\text { - Order a medication with harmful } \\
\text { drug interactions }\end{array}$ & $\begin{array}{l}\text { Review drug interaction information } \\
\text { and alerts } \\
\text { - Access external websites that provide } \\
\text { dedicated and detailed information on } \\
\text { drug interactions }\end{array}$ \\
\hline $\begin{array}{l}\text { Send } \\
\text { prescription } \\
\text { to pharmacy } \\
\text { to be filled }\end{array}$ & $\begin{array}{l}\text { Confirm that } \\
\text { prescription has } \\
\text { been sent to } \\
\text { pharmacy }\end{array}$ & $\begin{array}{l}\text { - Patient's preferred pharmacy might not } \\
\text { be listed in EHR } \\
\text { - EHR might contain incorrect contact } \\
\text { information for patient's preferred } \\
\text { pharmacy } \\
\text { - EHR might not confirm that prescription } \\
\text { was submitted to pharmacy }\end{array}$ & 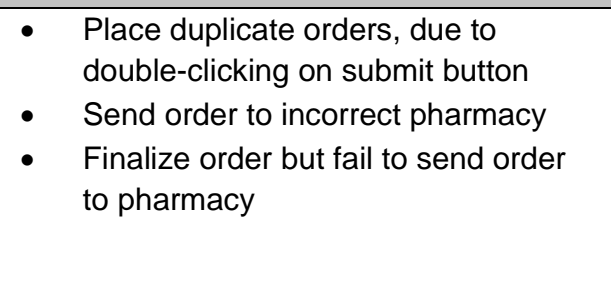 & $\begin{array}{l}\text { - } \quad \text { Verbally confirm pharmacy with patient } \\
\text { - } \quad \text { Confirm that prescription was } \\
\text { submitted to pharmacy }\end{array}$ \\
\hline
\end{tabular}




\subsubsection{Order procedure}

\begin{tabular}{|c|c|c|c|c|}
\hline Task & Challenge & Performance shaping factors & Potential use errors & Risk reduction strategies \\
\hline $\begin{array}{l}\text { Open patient } \\
\text { chart }\end{array}$ & Select correct patient & $\begin{array}{l}\text { - Clinicians see many patients } \\
\text { (perhaps } \geq 10 \text { ) in a single day } \\
\text { - Clinicians might wish to keep multiple } \\
\text { patient charts open at once } \\
\text { - Some patients might have identical or } \\
\text { similar names }\end{array}$ & $\begin{array}{l}\text { - Open wrong patient chart } \\
\text { - Confuse one patient's chart for } \\
\text { another }\end{array}$ & $\begin{array}{l}\text { - Confirm patient identity by } \\
\text { reviewing at least two patient } \\
\text { identifiers (e.g., D.O.B., full name, } \\
\text { photo) } \\
\text { - Constrain the number of patient } \\
\text { charts that might remain open at } \\
\text { once }\end{array}$ \\
\hline $\begin{array}{l}\text { Access ordering } \\
\text { module }\end{array}$ & Locate ordering module & $\begin{array}{l}\text { The patient chart's home screen } \\
\text { features relatively high information } \\
\text { density } \\
\text { - Exposure to other EHR content } \\
\text { organization schemes can lead to } \\
\text { negative transfer } \\
\text { - Providers might have multiple patient } \\
\text { chart and order windows open } \\
\text { simultaneously }\end{array}$ & $\begin{array}{l}\text { Open wrong module due to } \\
\text { confusing nomenclature or } \\
\text { content organization (e.g., } \\
\text { opening prescription ordering } \\
\text { module rather than procedure } \\
\text { ordering module) } \\
\text { Open wrong patient's procedure } \\
\text { ordering module }\end{array}$ & $\begin{array}{ll}\text { - } & \text { Interpret navigation cues by } \\
\text { utilizing icon tooltips } \\
\text { - } \quad \text { Verify name on patient ordering } \\
\text { module }\end{array}$ \\
\hline $\begin{array}{l}\text { Search for } \\
\text { procedure }\end{array}$ & $\begin{array}{l}\text { Search for correct } \\
\text { procedure }\end{array}$ & $\begin{array}{l}\text { - Clinicians might confuse procedures } \\
\text { with similar names, spellings or } \\
\text { descriptions (i.e., TSH vs. TSI test) } \\
\text { - Procedures might be listed under } \\
\text { different name than expected due to } \\
\text { multiple or overlapping clinical } \\
\text { terminology } \\
\text { - Procedures might be listed as } \\
\text { unfamiliar abbreviations } \\
\text { Procedure names and descriptions } \\
\text { might be long, complex and can } \\
\text { change over time (i.e., due to } \\
\text { innovation) } \\
\text { Clinicians might not distinguish } \\
\text { between inclusive (i.e., result must }\end{array}$ & $\begin{array}{l}\text { - Type procedure name into search } \\
\text { field incorrectly } \\
\text { - Select an incorrect procedure due } \\
\text { to similar name, spelling, or } \\
\text { description } \\
\text { - Inadvertently click on wrong } \\
\text { procedure (i.e., selection } \\
\text { accuracy) } \\
\text { Order procedure for wrong patient } \\
\text { (i.e., if wrong patient chart } \\
\text { selected previously) }\end{array}$ & $\begin{array}{l}\text { - Search for unfamiliar terms and } \\
\text { abbreviations by referring to an } \\
\text { external website or resource } \\
\text { Confirm procedure name and } \\
\text { details are appropriate after } \\
\text { selection }\end{array}$ \\
\hline
\end{tabular}




\begin{tabular}{|c|c|c|c|c|c|}
\hline & & & $\begin{array}{l}\text { include all search terms) and } \\
\text { exclusive search, thereby potentially } \\
\text { over-specifying the search term such } \\
\text { that the desired procedure does not } \\
\text { appear in the search results }\end{array}$ & & \\
\hline $\begin{array}{l}\text { Add diagnosis } \\
\operatorname{code}(s)\end{array}$ & $\begin{array}{l}\text { Select correct diagnosis } \\
\text { code(s) }\end{array}$ & & $\begin{array}{l}\text { Clinicians might not know which ICD } \\
\text { codes will be accepted by the } \\
\text { patient's insurance } \\
\text { Similar diagnoses might not be well- } \\
\text { distinguished in ICD code list or } \\
\text { database } \\
\text { Available ICD-9 codes might not be } \\
\text { sufficiently specific } \\
\text { The ICD-9 database is comprised of } \\
\text { over } 14,000 \text { diagnosis codes, and the } \\
\text { ICD-10 database is comprised of over } \\
68,000 \text { diagnosis codes } \\
\text { Patient's diagnosis list might not be } \\
\text { up-to-date } \\
\text { Procedure was ordered to determine } \\
\text { diagnosis (i.e., rapid flu test), so } \\
\text { clinician does not know which } \\
\text { diagnosis code is appropriate }\end{array}$ & $\begin{array}{l}\text { Enter an incorrect, unrelated, or } \\
\text { non-billable diagnosis code } \\
\text { - Incorrectly enter diagnosis name } \\
\text { when diagnosis code field calls for } \\
\text { ICD code, or vice versa } \\
\text { - Inadvertently click on wrong } \\
\text { diagnosis code (i.e., selection } \\
\text { accuracy) }\end{array}$ & $\begin{array}{l}\text { - Rely on previously memorized } \\
\text { ICD codes } \\
\text { - Search for unfamiliar diagnoses or } \\
\text { ICD codes by referring to an } \\
\text { external website or application } \\
\text { - Verify diagnosis code after } \\
\text { selection }\end{array}$ \\
\hline $\begin{array}{l}\text { Submit } \\
\text { procedure order }\end{array}$ & $\begin{array}{l}\text { Confirm that order has } \\
\text { been submitted }\end{array}$ & & $\begin{array}{l}\text { Submit button might be relatively } \\
\text { inconspicuous due to location } \\
\text { Submit button might not seem to } \\
\text { afford clicking } \\
\text { Submit button might not be clearly } \\
\text { labelled } \\
\text { EHR might not provide feedback } \\
\text { confirming order submission }\end{array}$ & $\begin{array}{l}\text { Exit order before submitting order } \\
\text { - Save order details but failing to } \\
\text { submit order } \\
\text { Submit order for wrong patient } \\
\text { (i.e., if wrong patient chart } \\
\text { selected previously) } \\
\text { - Place two duplicate orders, due to } \\
\text { double-clicking on submit button }\end{array}$ & $\begin{array}{l}\text { - Scan available buttons for } \\
\text { "submit" or synonymous terms } \\
\text { - Scan screen for confirmation } \\
\text { message after submitting order } \\
\text { - Check patient's chart to verify that } \\
\text { order appears in pending order list }\end{array}$ \\
\hline
\end{tabular}




\subsubsection{Manage problem list}

\begin{tabular}{|c|c|c|c|c|}
\hline Task & Challenge & Performance shaping factors & Potential use errors & Risk reduction strategies \\
\hline Open patient chart & Select correct patient & $\begin{array}{l}\text { - Clinicians see many patients } \\
\text { (perhaps } \geq 10 \text { ) in a single day } \\
\text { - Clinicians might wish to keep } \\
\text { multiple patient charts open at } \\
\text { once } \\
\text { - Some patients might have } \\
\text { identical or similar names }\end{array}$ & $\begin{array}{l}\text { - Open wrong patient chart } \\
\text { - } \quad \text { Confuse one patient's chart for another }\end{array}$ & $\begin{array}{l}\text { - } \quad \text { Confirm patient identity } \\
\text { by reviewing at least two } \\
\text { patient identifiers (e.g., } \\
\text { D.O.B., full name, photo) } \\
\text { Constrain the number of } \\
\text { patient charts that might } \\
\text { remain open at once }\end{array}$ \\
\hline $\begin{array}{l}\text { Access problem } \\
\text { list }\end{array}$ & Locate problem list & $\begin{array}{l}\text { The patient chart's home screen } \\
\text { features relatively high } \\
\text { information density } \\
\text { - Exposure to other EHR content } \\
\text { organization schemes can lead to } \\
\text { negative transfer } \\
\text { - Multiple providers might try to } \\
\text { open the same patient chart } \\
\text { problem list at the same time }\end{array}$ & $\begin{array}{l}\text { - Open wrong module due to confusing } \\
\text { nomenclature or content organization }\end{array}$ & $\begin{array}{ll}\text { - } & \text { Interpret navigation cues } \\
\text { by utilizing icon tooltips } \\
\text { - } & \text { Verify patient name on } \\
\text { - } & \text { module heading }\end{array}$ \\
\hline $\begin{array}{l}\text { Add diagnosis } \\
\text { code(s) }\end{array}$ & $\begin{array}{l}\text { Select correct diagnosis } \\
\text { code(s) }\end{array}$ & $\begin{array}{l}\text { - Clinicians might not know which } \\
\text { ICD codes will be accepted by } \\
\text { the patient's insurance } \\
\text { - Similar diagnoses might not be } \\
\text { well-distinguished in ICD code } \\
\text { list or database } \\
\text { - Available ICD-9 codes might not } \\
\text { be sufficiently specific } \\
\text { - The ICD-9 database is comprised } \\
\text { of over } 14,000 \text { diagnosis codes, } \\
\text { and the ICD-10 database is } \\
\text { comprised of over } 68,000 \\
\text { diagnosis codes } \\
\text { - Patient's diagnosis list might not } \\
\text { be up-to-date } \\
\text { Clinicians might not know which } \\
\text { diagnosis code is appropriate } \\
\text { because the procedure was } \\
\text { ordered to determine diagnosis }\end{array}$ & $\begin{array}{l}\text { - Enter an incorrect, unrelated, or non-billable } \\
\text { diagnosis code } \\
\text { - Incorrectly enter diagnosis name when } \\
\text { diagnosis code field calls for ICD code, or } \\
\text { vice versa } \\
\text { - Inadvertently click on wrong diagnosis code } \\
\text { (i.e., selection accuracy) }\end{array}$ & $\begin{array}{l}\text { Rely on previously } \\
\text { memorized ICD codes } \\
\text { - Search for unfamiliar } \\
\text { diagnoses or ICD codes } \\
\text { by referring to an } \\
\text { external website or } \\
\text { application } \\
\text { Verify diagnosis code } \\
\text { after selection }\end{array}$ \\
\hline
\end{tabular}




\begin{tabular}{|c|c|c|c|c|}
\hline \multicolumn{5}{|c|}{ (i.e., rapid flu test) } \\
\hline Audit problem list & $\begin{array}{l}\text { Maintain comprehensive } \\
\text { problem list }\end{array}$ & 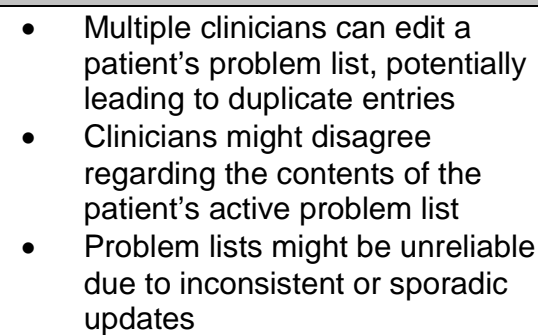 & $\begin{array}{l}\text { Delete rather than de-activate an entry, } \\
\text { thereby removing important information } \\
\text { from the patient's medical history } \\
\text { Neglect to update the patient's problem list } \\
\text { Inadvertently inactivate or re-activate } \\
\text { entries }\end{array}$ & $\begin{array}{l}\text { - Refer to organizational } \\
\text { policies regarding } \\
\text { problem list content } \\
\text { Review and update } \\
\text { problem list with patient } \\
\text { at the beginning or end } \\
\text { of each encounter } \\
\text { - Annotate problem list } \\
\text { entries }\end{array}$ \\
\hline $\begin{array}{l}\text { Save changes to } \\
\text { problem list }\end{array}$ & $\begin{array}{l}\text { Confirm that problem list } \\
\text { has been updated }\end{array}$ & $\begin{array}{l}\text { - Save button might be relatively } \\
\text { inconspicuous due to location } \\
\text { - Save button might not seem to } \\
\text { afford clicking } \\
\text { - Save button might not be clearly } \\
\text { labelled } \\
\text { - EHR might not provide feedback } \\
\text { confirming problem list update }\end{array}$ & $\begin{array}{l}\text { Exit problem list before saving changes } \\
\text { - Update wrong patient's problem list (i.e., if } \\
\text { wrong patient chart selected previously) }\end{array}$ & $\begin{array}{l}\text { Rely on internal } \\
\text { consistency in EHR to } \\
\text { guide user to save } \\
\text { problem list } \\
\text { Scan available buttons } \\
\text { for "save" or synonymous } \\
\text { terms } \\
\text { Scan screen for } \\
\text { confirmation message } \\
\text { after updating problem } \\
\text { list } \\
\text { Check patient chart after } \\
\text { saving to verify that } \\
\text { problem list was updated }\end{array}$ \\
\hline
\end{tabular}




\subsubsection{Enter vital signs}

\begin{tabular}{|c|c|c|c|c|}
\hline Task & Challenge & Performance shaping factors & Potential use errors & Risk reduction strategies \\
\hline Open patient chart & Select correct patient & $\begin{array}{l}\text { Clinicians see many patients } \\
\text { (perhaps } \geq 10 \text { ) in a single day } \\
\text { Clinicians might wish to keep } \\
\text { multiple patient charts open at } \\
\text { once } \\
\text { - Some patients might have } \\
\text { identical or similar names }\end{array}$ & $\begin{array}{l}\text { - } \quad \text { Open wrong patient chart } \\
\text { - } \quad \text { Confuse one patient's chart for another }\end{array}$ & $\begin{array}{l}\text { Confirm patient identity } \\
\text { by reviewing at least two } \\
\text { patient identifiers (e.g., } \\
\text { D.O.B., full name, photo) } \\
\text { Constrain the number of } \\
\text { patient charts that might } \\
\text { remain open at once }\end{array}$ \\
\hline $\begin{array}{l}\text { Access vital signs } \\
\text { module }\end{array}$ & $\begin{array}{l}\text { Locate vital signs } \\
\text { module }\end{array}$ & $\begin{array}{l}\text { The patient chart's home screen } \\
\text { features relatively high } \\
\text { information density } \\
\text { - Exposure to other EHR content } \\
\text { organization schemes can lead to } \\
\text { negative transfer } \\
\text { - Providers might have multiple } \\
\text { patient chart and vital signs } \\
\text { windows open simultaneously }\end{array}$ & $\begin{array}{l}\text { Open wrong module due to confusing } \\
\text { nomenclature or content organization (e.g., } \\
\text { opening prescription ordering module rather } \\
\text { than procedure ordering module) } \\
\text { Open wrong patient's procedure ordering } \\
\text { module }\end{array}$ & $\begin{array}{l}\text { - Interpret navigation cues } \\
\text { by utilizing icon tooltips } \\
\text { - Confirm patient name on } \\
\text { vital signs module }\end{array}$ \\
\hline $\begin{array}{l}\text { Enter vital signs } \\
\text { with units }\end{array}$ & $\begin{array}{l}\text { Enter accurate vital sign } \\
\text { values and units }\end{array}$ & $\begin{array}{l}\text { Clinicians might measure patient's } \\
\text { vital signs in an order that does } \\
\text { not t match the vital sign data } \\
\text { fields' order } \\
\text { Clinical workflow typically requires } \\
\text { that vital signs are entered quickly } \\
\text { - Several vital signs (e.g., height, } \\
\text { temperature) might be recorded in } \\
\text { either English or metric units } \\
\text { Order of vital sign data fields } \\
\text { varies among EHRs } \\
\text { Blood pressure field presentation } \\
\text { varies among EHRs (i.e., one vs. } \\
\text { two fields for systolic and diastolic } \\
\text { BP) }\end{array}$ & $\begin{array}{l}\text { Incorrectly enter vital sign measurements } \\
\text { (e.g., misplace a decimal point, omit digits, } \\
\text { enter one vital sign in another vital sign's } \\
\text { data field) } \\
\text { - } \quad \text { Enter incorrect units } \\
\text { Select incorrect units or neglect to modify } \\
\text { default units } \\
\text { Omit one or more vital signs fields }\end{array}$ & $\begin{array}{l}\text { - } \text { Confirm vital sign } \\
\text { measurements and units } \\
\text { seem appropriate in } \\
\text { context } \\
\text { - Compare vital sign } \\
\text { entries to past results and } \\
\text { normal ranges to ensure } \\
\text { results are written } \\
\text { correctly and in range (if } \\
\text { EHR does not do so } \\
\text { automatically) } \\
\text { Review alerts regarding } \\
\text { abnormal (or impossible) } \\
\text { vital sign ranges or } \\
\text { potential data entry errors }\end{array}$ \\
\hline
\end{tabular}




\begin{tabular}{|c|c|c|c|c|}
\hline & & & & $\begin{array}{l}\text { and modify vital sign } \\
\text { fields if necessary }\end{array}$ \\
\hline Save vital signs & $\begin{array}{l}\text { Confirm that vital signs } \\
\text { have been updated }\end{array}$ & $\begin{array}{l}\text { - Save button might be relatively } \\
\text { inconspicuous due to location } \\
\text { - Save button might not seem to } \\
\text { afford clicking } \\
\text { - Save button might not be clearly } \\
\text { labelled } \\
\text { - EHR might not provide feedback } \\
\text { confirming that vital signs were } \\
\text { saved to patient's chart }\end{array}$ & $\begin{array}{l}\text { - Exit vital signs module before saving } \\
\text { changes } \\
\text { - Save vital signs to wrong patient's chart } \\
\text { (i.e., if wrong patient chart selected } \\
\text { previously) }\end{array}$ & $\begin{array}{l}\text { - Rely on internal } \\
\text { consistency in EHR to } \\
\text { guide user to save vital } \\
\text { sign entries } \\
\text { Scan available buttons } \\
\text { for "save" or synonymous } \\
\text { terms } \\
\text { - Scan screen for } \\
\text { confirmation message } \\
\text { after adding vital signs } \\
\text { - Review patient chart after } \\
\text { saving to verify that vital } \\
\text { signs were updated }\end{array}$ \\
\hline
\end{tabular}




\subsubsection{Schedule appointment}

\begin{tabular}{|c|c|c|c|c|}
\hline Task & Challenge & Performance shaping factors & Potential use errors & Risk reduction strategies \\
\hline Open calendar & $\begin{array}{l}\text { Access calendar or } \\
\text { scheduling module }\end{array}$ & $\begin{array}{l}\text { EHRs vary as to whether clinician } \\
\text { or patient calendars are the } \\
\text { organizing structure for scheduling } \\
\text { Clinicians or medical assistants } \\
\text { might wish to open multiple } \\
\text { patient charts and/or calendars } \\
\text { simultaneously }\end{array}$ & $\begin{array}{l}\text { Open wrong provider's calendar } \\
\text { - Confuse one provider's calendar for } \\
\text { another }\end{array}$ & $\begin{array}{l}\text { - Confirm provider name } \\
\text { upon opening calendar } \\
\text { - If scheduling in patient } \\
\text { calendar, confirm patient } \\
\text { identify by reviewing at } \\
\text { least two patient identifiers } \\
\text { (e.g., D.O.B., full name, } \\
\text { photo) } \\
\text { Constrain the number of } \\
\text { patient charts that might } \\
\text { remain open at once }\end{array}$ \\
\hline $\begin{array}{l}\text { Search for } \\
\text { available } \\
\text { appointments }\end{array}$ & $\begin{array}{l}\text { Locate a mutually } \\
\text { convenient appointment }\end{array}$ & $\begin{array}{l}\text { EHR might not have straight- } \\
\text { forward or comprehensive } \\
\text { appointment search features } \\
\text { Appointments must fit within the } \\
\text { patient's schedule as well as the } \\
\text { clinician's schedule and criteria } \\
\text { (e.g., appointment length, } \\
\text { appointment type, timeframe, etc.) } \\
\text { EHR might not clearly indicate } \\
\text { whether an appointment search } \\
\text { yielded no results (i.e., as } \\
\text { opposed to loading issues or } \\
\text { incorrectly spelled patient or } \\
\text { clinician name) }\end{array}$ & $\begin{array}{l}\text { - Search for appointments in the wrong } \\
\text { patient's calendar } \\
\text { Incorrectly enter appointment search } \\
\text { parameters (e.g., misspell patient or } \\
\text { clinician names, search for wrong year or } \\
\text { date) } \\
\text { When appointment search yields no } \\
\text { results, incorrectly assume no } \\
\text { appointments are available rather than } \\
\text { confirming that appointment search } \\
\text { parameters are correct }\end{array}$ & $\begin{array}{l}\text { - } \quad \text { Verify appointment criteria } \\
\text { using notes from clinician } \\
\text { and patient input } \\
\text { - } \quad \text { Visually scan calendar for } \\
\text { available appointments } \\
\text { matching appointment } \\
\text { criteria }\end{array}$ \\
\hline
\end{tabular}




\begin{tabular}{|c|c|c|c|c|}
\hline $\begin{array}{l}\text { Select appointment } \\
\text { time and date }\end{array}$ & $\begin{array}{l}\text { Recall appointment } \\
\text { criteria }\end{array}$ & $\begin{array}{l}\text { Appointment selection is subject } \\
\text { to multiple criteria, including } \\
\text { appointment length, appointment } \\
\text { type, and timeframe } \\
\text { - Search results might be relatively } \\
\text { extensive and presented in a } \\
\text { visually overwhelming manner } \\
\text { hindering efficient visual scanning }\end{array}$ & $\begin{array}{l}\text { - Inadvertently click on wrong time slot (i.e., } \\
\text { selection accuracy) } \\
\text { - Schedule appointment for correct time on } \\
\text { incorrect date } \\
\text { - Schedule appointment for correct time } \\
\text { and date but incorrect year } \\
\text { - Schedule a patient for the wrong } \\
\text { appointment type (e.g., follow-up instead } \\
\text { of new patient appointment) } \\
\text { Inadvertently schedule multiple } \\
\text { appointments for a patient }\end{array}$ & $\begin{array}{l}\text { - Read and confirm selected } \\
\text { appointment with patient } \\
\text { - Compare selected } \\
\text { appointment time and date } \\
\text { to appointment criteria } \\
\text { provided to ensure selected } \\
\text { time is appropriate } \\
\text { - Review alerts regarding } \\
\text { abnormal (or impossible) } \\
\text { appointment options }\end{array}$ \\
\hline $\begin{array}{l}\text { Link appointment } \\
\text { to patient record }\end{array}$ & Select correct patient & $\begin{array}{l}\text { Clinicians or support staff might } \\
\text { have multiple patient charts open } \\
\text { simultaneously } \\
\text { - Some patients have identical or } \\
\text { similar names } \\
\text { - EHR might provide insufficient } \\
\text { feedback regarding whether } \\
\text { patient record has been } \\
\text { successfully linked to appointment }\end{array}$ & $\begin{array}{l}\text { - } \quad \text { Link appointment to an incorrect patient } \\
\text { - } \quad \text { Confuse one patient for another } \\
\text { Incorrectly assume that patient's } \\
\text { appointment was successfully linked to } \\
\text { patient record (i.e., if patient's name was } \\
\text { entered into a data field but not } \\
\text { recognized by the system) }\end{array}$ & $\begin{array}{l}\text { Confirm patient identify by } \\
\text { reviewing at least two } \\
\text { patient identifiers (e.g., } \\
\text { D.O.B., full name, } \\
\text { photo)Scan screen for } \\
\text { implicit or explicit cues that } \\
\text { patient record has been } \\
\text { successfully linked to } \\
\text { patient's appointment }\end{array}$ \\
\hline Save appointment & $\begin{array}{l}\text { Confirm that } \\
\text { appointment was saved }\end{array}$ & $\begin{array}{l}\text { - Save button might be relatively } \\
\text { inconspicuous due to location } \\
\text { - Save button might not seem to } \\
\text { afford clicking } \\
\text { - Save button might not be clearly } \\
\text { labelled } \\
\text { - EHR might not provide feedback } \\
\text { confirming that appointment was } \\
\text { successfully scheduled }\end{array}$ & $\begin{array}{l}\text { Exit appointment creation window or } \\
\text { schedule before saving changes } \\
\text { Schedule an appointment for the wrong } \\
\text { patient (i.e., if wrong patient chart selected } \\
\text { previously) }\end{array}$ & $\begin{array}{l}\text { Scan available buttons for } \\
\text { "save" or synonymous } \\
\text { terms } \\
\text { - Scan screen for } \\
\text { confirmation message after } \\
\text { saving appointment } \\
\text { Check patient or provider } \\
\text { schedule after saving to } \\
\text { verify that appointment was } \\
\text { scheduled } \\
\text { Compare newly scheduled } \\
\text { appointment's appearance } \\
\text { to previously scheduled } \\
\text { appointments }\end{array}$ \\
\hline
\end{tabular}




\subsection{Sample expert review findings}

Expert review findings are typically categorized as strengths or problems (i.e., opportunities for improvement), augmented by potential design improvements. This appendix presents several examples of expert review findings created in the context of the research study.

\section{Shortcoming: Placement of referral function}

Finding category: Content Organization

Issue: The referral placement (i.e., Referrals) function is categorized under the Demographics tab.

Potential consequences: Users might not find the Referral function because it does not seem related to Demographics.

Potential design improvement: Add a new tab titled Referral.

Figure 6-1: Referral function located under Demographics.

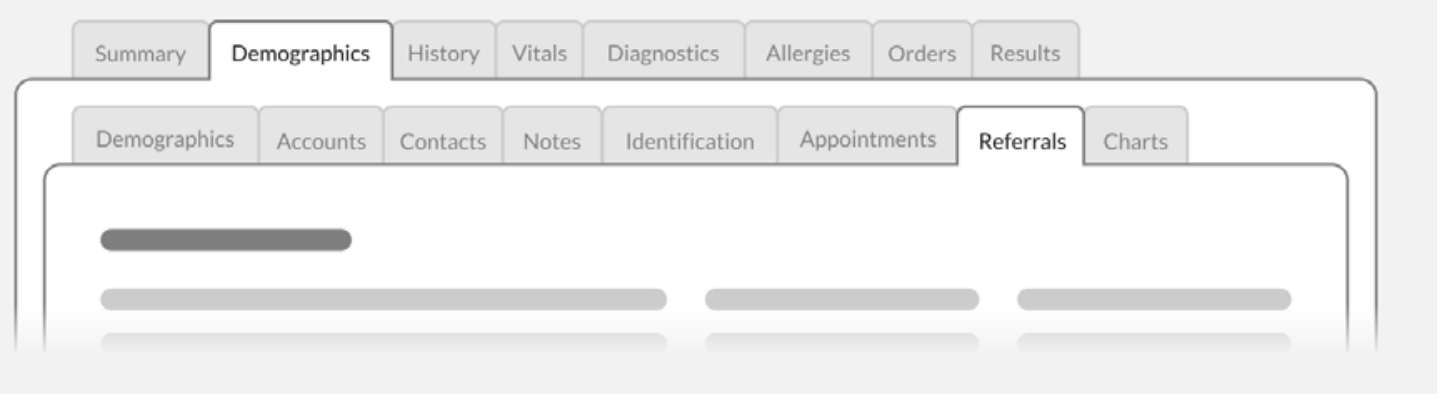

\section{Shortcoming: Use of ellipses}

Finding category: Affordances

Issue: The use of ellipses to indicate that the user can access more details about patient Allergies might not draw the user's attention because the symbol is neither informative nor conspicuous. Moreover, some users might not recognize whether the presence of ellipses is a positive indication that the patient has allergies or not; the symbol is ambiguous. The user should not have to navigate to a list of Allergies to determine that the patient has none.

Potential consequences: A user might overlook the fact that the patient has an allergy that has bearing on a diagnosis and/or treatment.

Potential design improvement: Replace the ellipses with an informative term, such as Yes that links to a list of Allergies. 
Figure 6-2: Indication of allergies is subtle.

Smith, Joanne

Gender: Female DOB: 12/12/1968 Age: 47 years Weight 232 lbs Allergies: ...

Figure 6-3: Clicking on “..." leads to list of allergies or none.

Smith, Joanne

Gender:Female

DOB: $12 / 12 / 1968$

Age: 47 years

Weight $232 \mathrm{lbs}$

Allergies: .h

Patient is allergic to pollen

\section{Strength: Allergy Alerts}

Finding category: Content Organization

Finding: The application alerts the HCP that the patient is allergic to pollen. Pollen is highlighted in red text to draw the user's attention.

Potential benefits: The HCP will be aware of ongoing health issues that might have bearing on patient diagnosis and treatment.

Figure 6-4: Clinical reminders.

\begin{tabular}{|c|c|c|}
\hline \multicolumn{3}{|l|}{ Smith, John } \\
\hline - Alerts and Reminders & & Edit \\
\hline - Appointments & & Add \\
\hline \multicolumn{3}{|l|}{$\begin{array}{l}\text { Tuesday, 2015-07-07 } \\
\text { 10:00 am Office Visit } \\
\text { Kim Brown }\end{array}$} \\
\hline v Medical Conditions & Add & Edit \\
\hline \multicolumn{3}{|l|}{ Asthma } \\
\hline \multicolumn{3}{|l|}{ Chronic Sinusitis } \\
\hline V Allergies & Add & Edit \\
\hline \multicolumn{3}{|l|}{ Pollen } \\
\hline v Medications & Edit & Print \\
\hline
\end{tabular}




\section{Appendix D: User Profiles of 10 EHR Users}

This section presents 10 sample user profiles for individuals who might interact with Electronic Health Records (EHRs). The samples cover the following occupational titles:

- Medical Assistant

- Medical Record and Health Information Technician

- Technologist and Technician

- Nurse

- Advanced Practice Registered Nurse

- Physician Assistant

- Anesthesia Care Provider

- Physician

- Surgeon

- Pharmacist

Note that the user profiles are samples (i.e., exemplars) and should not necessarily be used directly by EHR developers. Rather, an EHR developer should either (1) create original user profiles, perhaps guided by the format and general contents of the exemplars, or (2) at least adapt the exemplars based on its own research findings.

Also note that the 10 samples do not comprise a complete set. Depending on the EHR, there might be other types of users warranting their own user profiles. For example, there might be a need to develop user profiles matching the following occupational titles:

- Administrative assistants

- Billing specialists

- Emergency medical technicians

- Office managers

- Paramedics

- Therapists 
The user profiles describe characteristics deemed common among many individuals, but not necessarily all individuals sharing the same occupational title or belonging to what an EHR development team might describe as a distinct user group. The information categories included in the sample user profiles are the following:

- Occupational description

- Demographics (USA)

- Education

- Licensure/Certification

- Electronic Health Record (EHR) Use Environment(s)

- Experience using computers

- $\quad$ Sample EHR tasks

- Performance-shaping factors

Ultimately, EHR developers should include within user profiles the information that will be most useful to defining user interface requirements based on user needs and preferences and selecting participants for formative and summative evaluations (e.g., usability tests). The information might come from development project-specific research (e.g., user observations, user interviews), corporate knowledge about the users, findings from pertinent literature, and other possible sources.

While the following user profiles are 2-7 pages in length, a given profile might be considerably shorter or longer depending on the amount of pertinent information there is to share with readers.

\subsection{Medical Assistant}

\subsubsection{Occupational description}

Medical assistants (MAs) might carry out clinical as well as administrative tasks. Their duties vary depending on the location, specialty, and size of the practice. ${ }^{18}$ In larger practices or hospitals, MAs can specialize in either administrative or clinical work.

Some MAs specialize according to the type of office in which they work.

- Administrative MAs. Tasks include filling out insurance forms, coding patient medical information, answering telephone calls, and scheduling patient appointments.

\footnotetext{
${ }^{18}$ What Medical Assistants Do, http://www.bls.gov/ooh/healthcare/medical-assistants.htm
} 
- Clinical MAs. Duties vary depending on the state in which they work and the individual's training or certification. Tasks can include preparing patients for x-rays, drawing blood, assisting with activities of daily living (e.g., bathing, ambulation), obtaining basic medical information (e.g., why the patient is visiting the doctor), obtaining vital signs, and instructing patients about treatment plans.

\subsubsection{Demographics (USA)}

- Age: 37 years of age ${ }^{19}$

- Sex: Approximately $88 \%$ are female and $12 \%$ are male ${ }^{20}$

- Population: As of 2012 , there were around $560,800 \mathrm{MAs}^{21}$

\subsubsection{Education}

Most states lack formal educational requirements for becoming a medical assistant. MAs can graduate from post-secondary (i.e., after high school graduation) education programs or start medical assisting through on-the-job training. Although MAs are not required to graduate from post-secondary programs, most employers prefer to hire MAs who have completed such programs.

Programs for MAs span approximately one or two years, although some are considerably shorter (e.g., 6 weeks), and usually lead to a certificate, diploma, or associate degree. They are available at community and junior colleges, vocational schools, technical schools, and universities. Programs consist of both classroom and laboratory education with lessons in anatomy and medical terminology. Several states require MAs to graduate from an accredited program and/or pass an exam to do more advanced tasks, such as take $x$-rays and give injections.

In contrast, some MAs have a high school diploma (or the equivalent) and learn through on-thejob training. On-the-job training might include learning medical terminology and how to record information in and code electronic health records (EHRs).

\subsubsection{Licensure, certifications, and registrations}

Although MAs are not required to be certified, many employers prefer to hire certified assistants.

Several organizations offer certification that either requires the MA to pass an exam or graduate from an accredited program.

The National Commission for Certifying Agencies, part of the Institute for Credentialing Excellence, accredits five certifications for medical assistants:

- Certified Clinical Medical Assistant (CCMA) from the National Healthcareer Association

\footnotetext{
${ }^{19}$ Page 30 Figure 3-1. Page 31 first paragraph. EMS Workforce for the 21st Century: A National Assessment 30. http://www.ems.gov/pdf/emsworkforcereport june2008.pdf

${ }_{20}^{20}$ Page 21, http://bhpr.hrsa.gov/healthworkforce/supplydemand/usworkforce/chartbook/chartbook3.pdf

${ }^{21}$ Work Environment, http://www.bls.gov/ooh/healthcare/medical-assistants.htm
} 
- Certified Medical Administrative Assistant (CMAA) from the National Healthcareer Association

- Certified Medical Assistant (CMA) from the American Association of Medical Assistants (AAMA)

- National Certified Medical Assistant (NCMA) from the National Center for Competency Testing

- Registered Medical Assistant (RMA) from American Medical Technologists

\subsubsection{Electronic Health Record (EHR) Use environment(s)}

More than half of all MAs work in physician offices. ${ }^{22}$ According to a limited data collection effort in 2008 , the majority (62\%) of MAs worked in physician offices while small percentages of MAs worked in settings such as hospitals (13\%) and the offices of non-physician providers (11\%). ${ }^{23}$

Credentialed medical assistants are able to enter (i.e., transcribe) medication, radiology, and laboratory orders into the EHR in accordance with clinicians' orders. Their entry counts toward meeting the requirements for meaningful use for the Medicare and Medicaid EHR Incentive Programs. $^{24}$
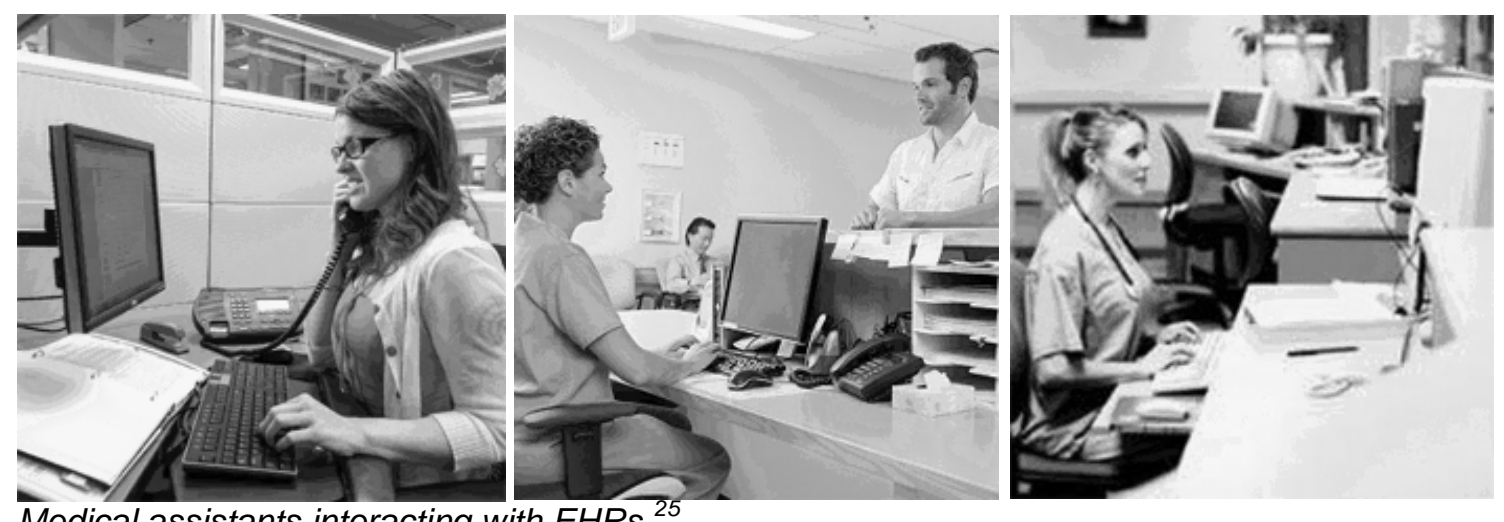

Medical assistants interacting with EHRs. ${ }^{25}$

\subsubsection{Experience using computers}

An MA's comfort level with computers might vary based on prior computer use and overall education.

Due to computer use starting in childhood, younger MAs are more likely to have experience with and be comfortable participating in computer-based training. MAs who used computers

\footnotetext{
${ }^{22}$ Work environment, http://www.bls.gov/ooh/healthcare/medical-assistants.htm\#tab-3

${ }^{23} \mathrm{http} / / / \mathrm{ww} w$. cuny.edu/about/administration/offices/hhs/CUNY.MAReport.2.6.12.pdf, page 3.

${ }_{24}^{24}$ Assessment-Based Recognition in Order Entry (ABR-OE), http://www.aama-ntl.org/medical-assisting/abr

${ }^{25}$ Photo sources: http://uminsideview.org/wp-content/uploads/2014/04/Corl.jpg, http://www.globeuniversity.edu/blogs/wpcontent/uploads/2014/10/bigstock-nurse-and-patient-conversing-a-56375318.jpg, http://www.pro-medtech.com/images/Medical_Assistant_Training.jpg
} 
throughout their schooling are generally more inclined to explore unfamiliar functions and perform tasks with high efficiency.

Often, younger MAs might also have a clearer mental model of computer-based data management, including uploading and downloading data and using computers to access other data sources. Those MAs that did not use computers during schooling are generally less comfortable exploring computer functions and in some cases perform tasks with less efficiency than younger MAs.

Typing speed is not necessarily a function of age. While younger MAs might have used computers since childhood and have become proficient typists, older MAs might have taken typing classes, which were more common in years past, and also become fast at data entry tasks due to their own cumulative years of interacting with computers.

\subsubsection{Sample EHR tasks}

- Create new patient records

- Add patient information to existing records, including demographic, billing and insurance information

- Schedule, reschedule, and cancel appointments

- Schedule referral appointments

- Print, fax, and electronically send provider signed orders for laboratory tests and procedures

- Document communication with other facilities (e.g., that an order has been faxed)

- Follow up with patients regarding upcoming appointments or follow-up care

- Document patient communication (e.g., a patient phone call)

- Locate clinician appointment and other follow-up care requests in patient records

- Enter vital signs (clinical MAs)

- Enter intake and output (clinical MAs) (e.g., oral fluid intake, urine output)

\subsubsection{Performance-shaping factors}

The following factors, related to occupational responsibility, training, and familiarity with computers, could be expected to influence MAs' interactions with EHRs:

- Fast-paced environments. Working in fast-paced work environments can create stress and fatigue, leading users to seek shortcuts and workarounds to complete required tasks in time available 
- Little time between patients. Lacking time between patients can make MAs more likely to delay entering data into the EHR. This would require them to rely more on memory to correctly recall and associate information and observations with the correct patient

- Working with multiple patients simultaneously. This requires MAs to rely more on memory to correctly recall and associate information and observations with the correct patient

- Need for clinician input. Confirming details or consulting with nurses and other healthcare professionals might delay work

- Alternating communication modes. Switching frequently between phone-based, paperbased, EHR-based, email, and in-person tasks and communication modes

- Excessive alerts and pop-ups. Encountering an extensive number of safety alerts and pop-ups might drive users to seek workarounds and ignore pop-ups

- Changing documentation modes. Shifting from documenting in paper charts to using a software-based solution might challenge familiar methods of documentation

- Lacking reference material. Lacking readily available reference material (e.g., user manual, quick reference guide) can lead the MA to rely on memory to recall operating procedures

- Interaction effect. Wearing protective equipment such as gloves might inhibit movement, visibility, and typing

\subsection{Medical Record and Health Information Technicians}

\subsubsection{Occupational description}

Medical Record and Health Information Technicians manage health information and data gathered from electronic health records (EHRs). Data analysts work to ensure the security, quality, accuracy, and accessibility of health information data.

Data analysts use various classification systems to collect, code, and categorize patient information. They often specialize in coding diagnoses using International Classification of Disease (ICD) codes and procedures using Current Procedural Terminology (CPT) codes. This information is imperative for insurance reimbursement, tracking specific types of diseases in databases and registries, and maintaining patients' medical and treatment histories. ${ }^{26}$

\subsubsection{Demographics (USA)}

- Age: $48.6 \%$ of data analysts are $35-55$ years old ${ }^{27}$

\footnotetext{
${ }^{26}$ Adapted from text found at http://www.bls.gov/ooh/healthcare/medical-records-and-health-informationtechnicians.htm\#tab-4

${ }^{27}$ Page 18, Figure 24, http://bhpr.hrsa.gov/healthworkforce/supplydemand/usworkforce/chartbook/chartbook3.pdf
} 
- Sex: Approximately $88.8 \%$ are female and $11.2 \%$ are male ${ }^{28}$

- Population: As of 2012, there were around 111,297 data analysts ${ }^{29}$

\subsubsection{Education}

Data analysts are encouraged to begin taking courses on health, computer science, math, and biology while in high school. Technicians can then earn an associate degree in health information technology or earn postsecondary certificates. Coursework in these postsecondary programs emphasizes the following areas:

- Medical terminology

- Anatomy and physiology

- Health data requirements and standards

- Classification and coding systems

- Healthcare reimbursement methods

- Healthcare statistics

- $\quad$ Computer systems ${ }^{30}$

\subsubsection{Certification}

Employers prefer to hire data analysts who have earned a professional certification. There are numerous certifications offered by several organizations in the field of health information. Common certifications include Registered Health Information Technician (RHIT), Registered Health Information Administrator (RHIA), and Certified Tumor Registrar (CTR) ${ }^{31}$ Some organizations base certification on passing an exam while others require graduation from an accredited program.

\subsubsection{Electronic Health Record (EHR) Use environment(s)}

Healthcare information technicians work primarily in hospitals, but also in office-based practices, long-term care facilities, and public health agencies. Additionally, facilities that use patient data or health information, such as pharmaceutical companies, insurance firms and health product vendors, might also need data analysts. ${ }^{32}$

\footnotetext{
${ }^{28}$ Page 18, Figure 24, http://bhpr.hrsa.gov/healthworkforce/supplydemand/usworkforce/chartbook/chartbook3.pdf

${ }^{29}$ Page 16, Figure 24, http://bhpr.hrsa.gov/healthworkforce/supplydemand/usworkforce/chartbook/chartbook3. pdf

${ }^{30}$ Adapted from text found at http://www.bls.gov/ooh/healthcare/medical-records-and-health-informationtechnicians.htm\#tab-4

${ }^{31} \mathrm{http}: / / \mathrm{www}$.bls.gov/ooh/healthcare/medical-records-and-health-information-technicians.htm\#tab-4

${ }^{32}$ Adapted from text found at http://www.ahima.org/certification/RHIT
} 


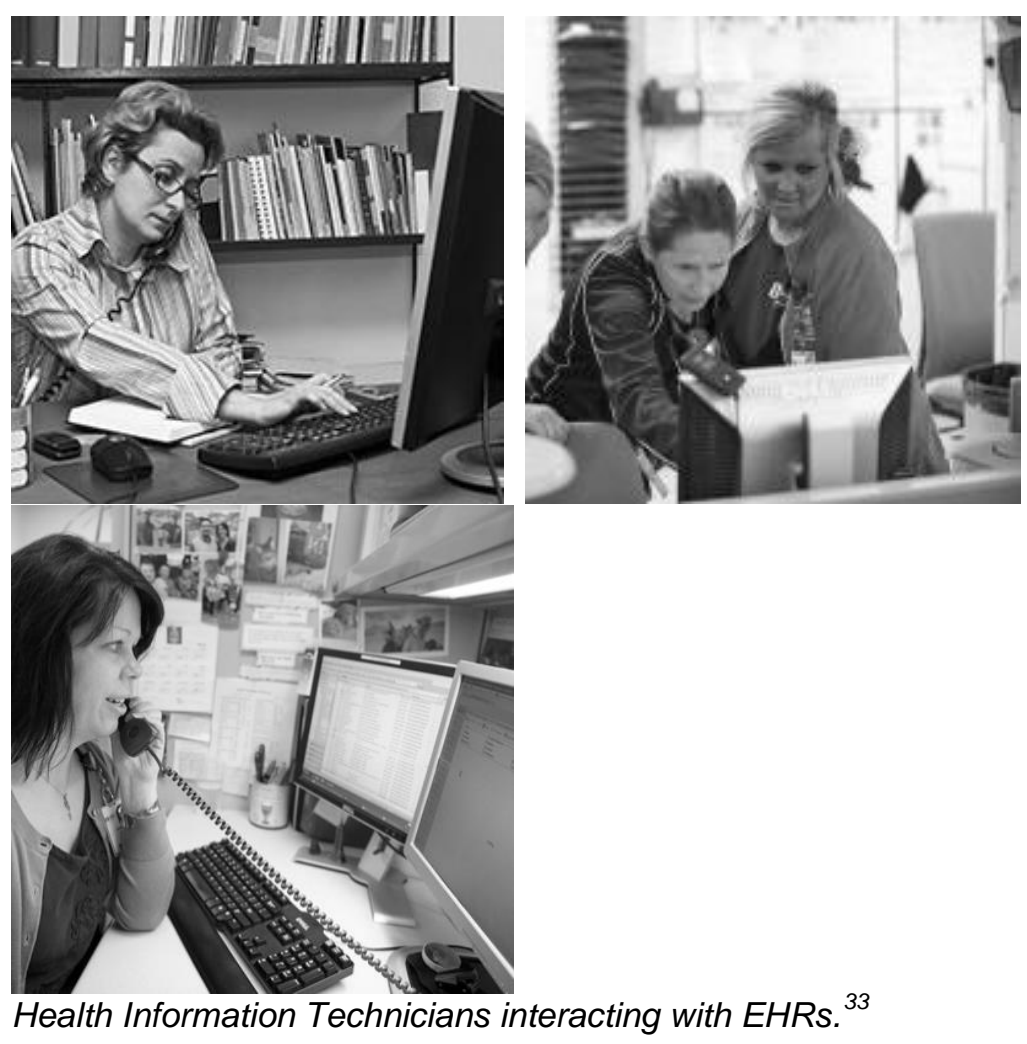

\subsubsection{Experience using computers}

Data analysts work with computers all day long. Thus, they typically have a high comfort level with computers and are more likely to have a clear mental model of computer-based data management, including uploading and downloading data and using computers to access other data sources. Those data analysts who did not use computers during schooling might be less comfortable exploring computer functions might perform some tasks with less efficiency.

\subsubsection{Sample EHR tasks}

- Retrieve patient records for medical personnel

- Work as a liaison between the health clinician and billing offices

- Review patient information for preexisting conditions

- Ensure patient records and reports are accurate and complete

- Assign correct classification codes (e.g., ICD-10 codes) to represent diagnosis and treatment

- Analyze and compile patient information for cancer registries and research purposes

\footnotetext{
${ }^{33}$ Photo sources: http://www.academyofmedicalprofessions.com/hospitalbillingandcoding.asp, http://inside.akronchildrens.org/2013/05/22/a-day-in-the-life-medical-coders/, http://inside.akronchildrens.org/2013/05/22/a-day-in-the-life-medical-coders/
} 
- Maintain facility, regional, and national databases of patients

- Ensure diagnoses and procedures are coded correctly and submitted for billing

- Track patient outcomes for quality assessment

- Communicate with providers to clarify diagnoses confirm accuracy of records

\subsubsection{Performance-shaping factors}

The following factors, related to occupational responsibility, training, and familiarity with computers, are just some of those that can influence technicians' interactions with EHRs:

- Repetitive Tasks. Facing repetitive tasks and alerts can drive users to seek workarounds and ignore pop-ups

- Need for clinician input. Confirming details or consulting with clinicians with intensive schedules might delay work

- Staring at computer screens. Staring at computer screens for long periods of time reviewing information and, therefore, might use shortcuts and overlook key details

- Continually changing environments. Continually changing environments make it challenging to keep up - shifting from acquiring information and documenting on paper to using a software-based solution, as well as switching from ICD-9 codes to ICD-10 codes (mandatory after October 1, 2015)

- Alternating communication modes. Switching frequently between phone-based, paperbased, EHR-based, email, and in-person tasks and communication modes

- Excessive alerts and pop-ups. Encountering an extensive number of safety alerts and pop-ups might drive users to seek workarounds and ignore pop-ups

- Changing documentation modes. Shifting from documenting in paper charts to using a software-based solution might challenge familiar methods of documentation

- Lacking reference material. Lacking readily available reference material forces the data analyst to rely on memory to recall procedures and instructions

\subsection{Technologists and Technicians}

\subsubsection{Occupational description}

A technologist or technician's occupational responsibility is based on his/her specialty and the environment in which s/he works. Technicians often perform routine tests, while technologists carry out more complicated tasks and supervise technicians. Technologists and technicians in large laboratories are inclined to specialize, while technologists and technicians in small laboratories perform a variety of tasks and procedures. 
We focus on the following types of specialized technologists in this profile:

- Medical laboratory technologists and medical laboratory technicians collect samples and perform medical laboratory tests on body fluids and tissues ${ }^{34}$

- Radiologic technologists use imaging techniques to perform medical imaging exams and administer radiation therapy treatments ${ }^{35}$

\subsubsection{Demographics (USA)}

- Age: Average in the USA is 43 years for technologists ${ }^{36,37}$

- Sex: Approximately $72 \%$ are female, $28 \%$ are male (as of 2010$)^{38}$

- Population: 325,800 medical and clinical laboratory technologists and technicians (as of $2012)^{39}$ and 229,300 radiologic and magnetic resonance imaging technologists (as of 2012) $)^{40}$

\subsubsection{Education}

There are numerous pathways to become a technologist or a technician. Specific specialties have varying requirements for training. Outlined below are examples of ways that one might become a technologist or technician.

\subsubsection{Technologists}

Typically, entry-level medical technologists need to earn a bachelor's degree in life sciences or medical technology. An accredited bachelor's program in medical laboratory technology focuses on chemistry, biology, mathematics, and statistics, as well as laboratory skills and management. ${ }^{41}$

Many states require radiologic technologists to complete a postsecondary education program accredited by the Joint Review Committee on Education in Radiologic Technology in radiography or MRI to earn a license. Graduates of such postsecondary programs can leave with graduate certificates, associate degrees, or bachelor's degrees. Associate degree programs that include both classroom and clinical training are the most common. ${ }^{42}$

\subsubsection{Technicians}

Medical laboratory technicians must complete an associate degree program in clinical laboratory science or a related field. Technicians can enter the field by earning certificates through the

\footnotetext{
${ }^{34}$ http://www.bls.gov/ooh/healthcare/medical-and-clinical-laboratory-technologists-and-technicians.htm

${ }^{35}$ Adapted from text found at http://money.usnews.com/careers/best-jobs/radiologic-technologist

${ }^{36} \mathrm{http} / / / \mathrm{www}$.asrt.org/docs/default-source/research/r13_wagesalarysurvey.pdf?sfvrsn=2 and

${ }^{37} \mathrm{http}: / / \mathrm{www}$.ascp.org/pdf/MedicalLaboratoryPersonnelShortage.aspx

${ }^{38} \mathrm{http}: / /$ www.asrt.org/docs/default-source/research/r13_wagesalarysurvey.pdf?sfvrsn=2

${ }^{39} \mathrm{http} / / / \mathrm{www}$.bls.gov/ooh/healthcare/medical-and-clinical-laboratory-technologists-and-technicians.htm\#tab-1

${ }^{40} \mathrm{http} / / / \mathrm{www}$.bls.gov/ooh/healthcare/radiologic-technologists.htm\#tab-1

${ }^{41}$ Adapted from text found at http://www.bls.gov/ooh/healthcare/medical-and-clinical-laboratory-technologists-andtechnicians.htm\#tab-4

${ }^{42}$ Adapted from text found at http://www.bls.gov/ooh/healthcare/radiologic-technologists.htm\#tab-4
} 
armed forces, vocational or technical schools, or hospitals programs. Expedited certificate programs exist for those who already have a degree in a related field, such as nursing. ${ }^{43}$

\subsubsection{Licensure, certifications, and registrations}

Licensure, certification, and registration requirements vary by state, specialty, and employer. To earn a license, a technologist or technician often needs to have graduated from an accredited program and passed an exam. ${ }^{44}$ The American Society for Clinical Pathology Board of Certification provides certification for laboratory personnel, including medical laboratory scientists (MLS) and medical laboratory technicians (MLT).

\subsubsection{Electronic Records (EHR) Use environment(s)}

More than half of all technologists and technicians work in medical and surgical hospitals. Other sites of employment include physician offices, medical and diagnostic laboratories, and outpatient care centers.

Medical and clinical laboratory technologists and technicians often wear protective masks, gloves, and eye protection when working with infectious specimens or flammable materials. ${ }^{45}$ Radiologic technologists minimize the risk of radiation hazards by using lead aprons, gloves, and other shielding devices, while also monitoring exposure to radiation. ${ }^{46}$
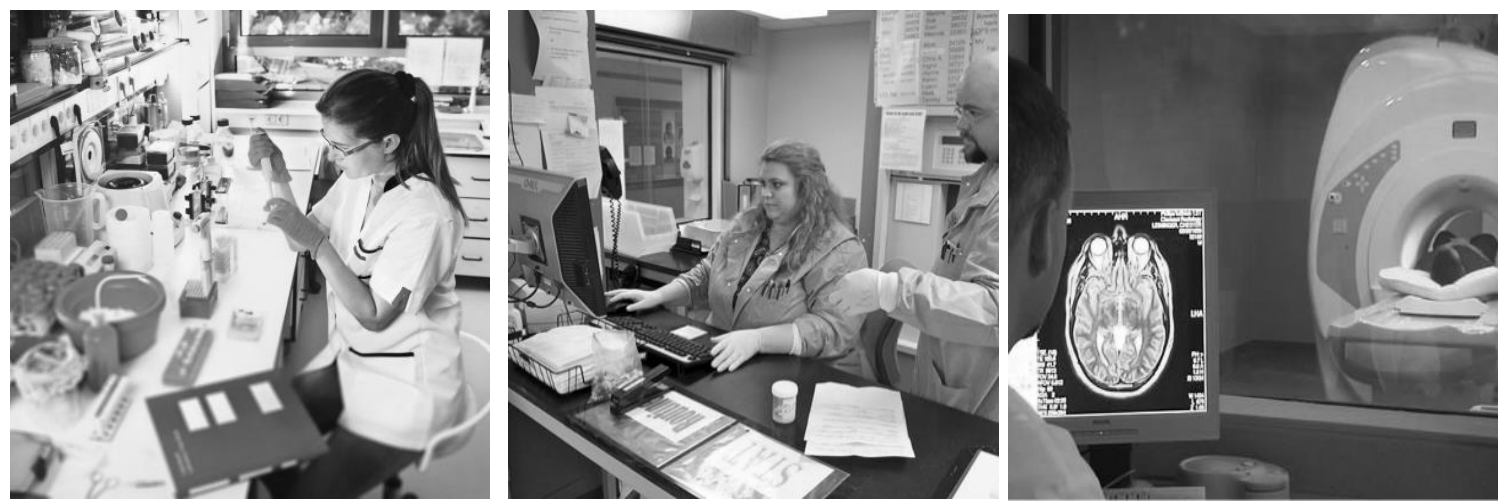

Medical, clinical, and radiologic technicians and technologists interacting with electronic health records (EHRs). ${ }^{47}$

\subsubsection{Experience using computers}

In general, technicians and technologists are bimodal in their use of computers and EHRs. This largely depends on whether the technician or technologist used computers during his/her

\footnotetext{
${ }^{43}$ Adapted from text found at http://www.bls.gov/ooh/healthcare/medical-and-clinical-laboratory-technologists-andtechnicians.htm\#tab-4

${ }^{44}$ Adapted from text found at http://www.bls.gov/ooh/healthcare/medical-and-clinical-laboratory-technologists-andtechnicians.htm\#tab-4

${ }^{45}$ Adapted from text found at http://www.bls.gov/ooh/healthcare/medical-and-clinical-laboratory-technologists-andtechnicians.htm\#tab-3

${ }^{46}$ Adapted from text found at http://www.bls.gov/ooh/healthcare/radiologic-technologists.htm\#tab-3

${ }^{47}$ Photo sources: http://aphealthrecords.com/portfolio_ibusiness/laboratory/, http://inside.akronchildrens.org/2013/04/23/a-day-in-the-life-medical-lab-technician/, $\mathrm{http} / / / \mathrm{www}$.amberusa.com/dmxreadyv2/blogmanager/blogmanager.asp?category=37\&pageindex=7
} 
education. Thus, older technicians or technologists are generally less comfortable with computers and EHRs.

Due to computer use starting in childhood, younger technicians or technologists are more likely to have experience with and be comfortable participating in computer-based training. Technicians or technologists who used computers throughout their schooling are generally more inclined to explore unfamiliar functions and perform tasks with high efficiency.

Younger technicians or technologists might also have a clearer mental model of computer-based data management, including uploading and downloading data and using computers to access other data sources. Those who did not use computers during schooling are generally less comfortable exploring computer functions and in some cases perform tasks with less efficiency.

Typing speed is not necessarily a function of age. While younger technicians or technologists might have used computers since childhood and have become proficient typists, older technicians or technologists might have taken typing classes, which were more common in years past, and also become fast at data entry tasks due to their own cumulative years of interacting with computers.

\subsubsection{Sample EHR tasks}

- Read and carry out orders for diagnostic tests or procedures

- $\quad$ Prepare patients for procedures (e.g., record medical history)

- Document care, such as administering a test or procedure

- Communicate with physicians to evaluate the images or samples

- Print, fax, and send orders and results for laboratory tests and procedures

- Document communication with other facilities (e.g., that an order has been faxed)

- Record data from medical tests and enter results into a patient's record ${ }^{48}$

\subsubsection{Performance-shaping factors}

The following factors, related to occupational responsibility, training, and familiarity with computers, could be expected to influence technicians' and technologists' interactions with EHRs:

- Fast-paced environments. Working in fast-paced work environments can create stress and fatigue, leading technicians and technologists to seek shortcuts and workarounds to complete required tasks in time available

- Production pressures. Technicians and technologists can face significant production pressure in their daily work. For example, insufficient time between patients to perform

\footnotetext{
${ }^{48}$ Adapted from text found at http://www.bls.gov/ooh/healthcare/medical-and-clinical-laboratory-technologists-andtechnicians.htm\#tab-2
} 
tests or procedures can make technicians and technologists more likely to avoid seeking information, to delay entering data into the EHR or to enter less information than they would otherwise. Such behaviors could require them to rely more on memory to correctly recall and associate information and observations about a patient

- Need for clinician input. Confirming details or consulting with physicians or nurses who are difficult to reach might delay work and contribute to production pressure

- Alternating communication modes. Switching frequently between phone-based, paperbased, EHR-based, email, and in-person tasks and communication modes

- Excessive alerts and pop-ups. Encountering an extensive number of safety alerts and pop-ups might drive users to seek workarounds and ignore pop-ups

- Developing alarm fatigue. Developing alarm fatigue can lead to ignored or missed audible alarms, as well as a disdain for onscreen warnings

- Changing documentation modes. Shifting from documenting in paper charts to using a software-based solution might challenge familiar methods of documentation

- Lacking reference material. Lacking readily available reference materials can make technicians and technologists rely on memory to recall procedures and instructions

- Interaction effect. Wearing protective equipment such as gloves can inhibit movement, visibility, and typing

- Stress of dealing with difficult patients or situations. Under stressful conditions, technicians and technologists might be more likely to take shortcuts, miss relevant cues, or to become frustrated when presented with obstacles to achieving their clinical goals while using the EHR

\subsection{Nurse}

\subsubsection{Occupational description}

A nurse's role is based on his/her professional scope of practice (licensure), specialty field, experience, the environment in which s/he works, and the patients s/he serves. Nurses, including registered nurses (RNs), licensed practical nurses (LPNs), and licensed vocational nurses (LVNs), most often work as part of a team that includes physicians, other nurses, ancillary staff (e.g., respiratory therapists, physical therapists), and medical assistants. Some RNs oversee the work of other nurses, nursing assistants, and home health aides. An increasing number of RNs now work in roles outside the traditional direct patient care role, such as health systems management, research, and informatics. This profile specifically excludes advanced practice nurses (APN) such as nurse anesthetists, nurse midwives, and nurse practitioners (all of whom must first be RNs).

A nurse's work might focus on one or more of the following specific fields: 
- A health condition, such as diabetes

- A body part, such as the skin

- A group of people, such as the elderly

- A workplace, such as an emergency department in a hospital

Some nursing tasks common to most nurses are the following:

- Consult with doctors and other healthcare professionals

- Record patients' medical histories, symptoms, and vital signs

- Administer patients' medicines and treatments

- Contribute to plans for patient care

- Coordinate patient care in collaboration with other healthcare professionals

- Observe patients and record observations

- Operate medical equipment

- Help perform diagnostic tests and analyze results

- Serve as an interface and resource between patients and their families, and healthcare providers and other elements of the healthcare system

- Teach patients and their families how to manage illnesses or injuries

RNs provide a more advanced level of professional nursing care than do LVNs or LPNs. RNs structure their work around the nursing process, which involves assessing a wide range of factors that impact a patient's health and well-being (e.g., physiological, psychosocial, spiritual, and environmental factors) and applying clinical judgment to identify a patient's problems or potential problems. ${ }^{49}$ Based on this ongoing assessment and nursing diagnosis process, RNs develop and implement a nursing plan of care to address the individual patient's needs and evaluate patient outcomes.

\subsubsection{Demographics (USA)}

- Age: Average ages are 44.6 years for RNs and 43.6 years for LPNs ${ }^{50}$

- Sex: Approximately $91 \%$ of nurses are female (as of 2011$)^{51}$

- Population: $3,063,163$ RNs as of $2008^{52}$ and 738,400 LPNs and LVNs as of $2012^{53}$

\footnotetext{
${ }^{49} \mathrm{http}$ ///www.nursingworld.org/EspeciallyForYou/What-is-Nursing/Tools-You-Need/Thenursingprocess.html

${ }^{50} \mathrm{HRSA}$ analysis of the ACS 2008-2010 three-year file; HRSA analysis of the ACS 2008-2010 three-year file

${ }^{51}$ HRSA analysis of the ACS 2008-2010 three-year file; HRSA analysis of the ACS 2008-2010 three-year file
} 


\subsubsection{Education}

There are numerous pathways to becoming a nurse. Becoming an RN requires the completion of a post-secondary education program and earning an associate or bachelor's degree. Very few hospitals still have teaching/training programs that offer diplomas. Aspiring nurses learn about a wide breadth of topics, including anatomy and human development, and gain clinical experience through progressive, supervised practice.

\subsubsection{LPNs and LVNs}

LPNs and LVNs provide basic or routine nursing care under the clinical supervision of an RN, APN, or MD. LPNs and LVNs require a certificate or diploma from programs typically found in technical schools and community colleges. The programs span a year or more and include coursework and supervised clinical experiences. Common courses include:

- Pharmacology

- Nursing

- Biology

\subsubsection{Associate Degree in Nursing}

Many RNs enter the profession after earning an Associate Degree in Nursing (ADN) or Associate of Science in Nursing (ASN). Associate degree programs typically span 2-3 years and are available at community and vocational colleges. The programs include classroom instruction to prepare nurses for generalist practice, as well as hands-on training in hospitals, clinics, or other healthcare settings.

Common courses include:

- Adult and family health

- Psychiatric nursing

- Pharmacology

- Human development

- Anatomy and physiology

According to the U.S. Bureau of Labor Statistics (BLS), nurses who have earned associate degrees or diplomas often pursue bachelor's degrees later in their careers.

\subsubsection{Bachelor of Science in Nursing}

\footnotetext{
${ }^{52}$ http://bhpr.hrsa.gov/healthworkforce/rnsurveys/rnsurveyinitial2008.pdf

${ }^{53}$ http://bhpr.hrsa.gov/healthworkforce/reports/nursingworkforce/nursingworkforcefullreport.pdf
} 
RNs can take a more comprehensive educational path by earning a Bachelor of Science in Nursing (BSN). Colleges and universities offer BSN programs that typically span four years. However, students already licensed as RNs or possessing a bachelor's degree in another field might complete accelerated programs.

BSN programs concentrate on more advanced nursing practices and clinical training than lowerlevel degree programs. They provide students with the administrative and critical thinking skills necessary for advanced positions in the field.

Common courses include:

- Community health

- Leadership in nursing

- Healthcare management

- Nursing research

- Health assessment

- Professional issues in nursing ${ }^{54}$

\subsubsection{Licensure}

To practice in a specific state, all nurses must earn and maintain a nursing license in that state or in a state participating in the multistate Nursing Licensure Compact. While licensure requirements vary by state, nurses must complete a state-approved training program and pass examinations by the National Council of State Boards of Nursing. Passing the National Council Licensure Examination for Registered Nurses (NCLEX-RN) is a requirement to work as an RN. The NCLEXRN covers topics like psychosocial and physiological integrity, health promotion, and infection control. All states require LPNs and LVNs to pass the National Council Licensure Examination (NCLEX-PN). Depending on the state, nurses might be required to meet additional licensure requirements.

\subsubsection{Electronic Health Record (EHR) Use Environment(s)}

Nurses work in a wide variety of environments, including hospitals, clinics, physicians' offices, home healthcare service organizations, nursing care facilities, schools, correctional facilities, and military healthcare settings. ${ }^{55}$ In almost all of these settings, nurses document patient information, clinical assessments, and patient interactions in EHR systems.

\footnotetext{
${ }^{54}$ Adapted from text found at

http://study.com/articles/Registered_Nurse_RN_Educational_Requirements_for_Registered_Nurses.html; internal reference to www.bls.gov and www.ncsbn.org and

${ }^{55}$ Adapted from text found at http://www.bls.gov/ooh/healthcare/registered-nurses.htm
} 


\subsubsection{LPNs and LVNs}

Licensed practical vocational nurses often work in nursing homes, long-term care facilities, hospitals, physician offices, and private homes. The following table outlines the work locations for these nurses in 2012.

\begin{tabular}{lc} 
Employment settings $^{\mathbf{5 6}}$ & $\mathbf{\%}^{\mathbf{5 7}}$ \\
\hline Nursing care facilities & 29 \\
Hospitals & 20 \\
Offices of physicians & 12 \\
Home health care services & 11 \\
Residential care facilities & 8
\end{tabular}

\subsubsection{Registered Nurses}

The following table provides the distribution of registered nurses working in various hospital settings. Note that nurses work in many more settings that those listed and that some work in more than one setting.

\begin{tabular}{lr} 
Hospital settings $^{58}$ & $\%$ \\
\hline General or specialty inpatient unit & 38 \\
Critical or intensive care unit & 21 \\
Perioperative (OR, pre- or post-op care unit) & 19 \\
Step down-transitional-progressive-telemetry & 12 \\
Emergency department & 11
\end{tabular}

The following table provides the distribution of RNs working in various non-hospital settings. Again, note that RNs work in many more settings that those listed and that some work in multiple settings.

\footnotetext{
${ }^{56}$ Data Source: http://www.bls.gov/ooh/healthcare/licensed-practical-and-licensed-vocational-nurses.htm\#tab-3

${ }^{57}$ Note: Table taken from the Bureau of Labor and Statistics. Values add up to $80 \%$ which likely excludes other work environments or non-responses from BLS report.

${ }^{58}$ Data source: 2008 National Sample Survey of Registered Nurses
} 


\begin{tabular}{ll} 
Non-hospital settings $^{\mathbf{5 9}}$ & \% \\
\hline Ambulatory care & 24 \\
Home health & 16 \\
Long-term care/skilled nursing facility & 14 \\
Business/administration/review & 14 \\
Public/community health & 12 \\
Education & 12 \\
General or specialty inpatient & 11
\end{tabular}
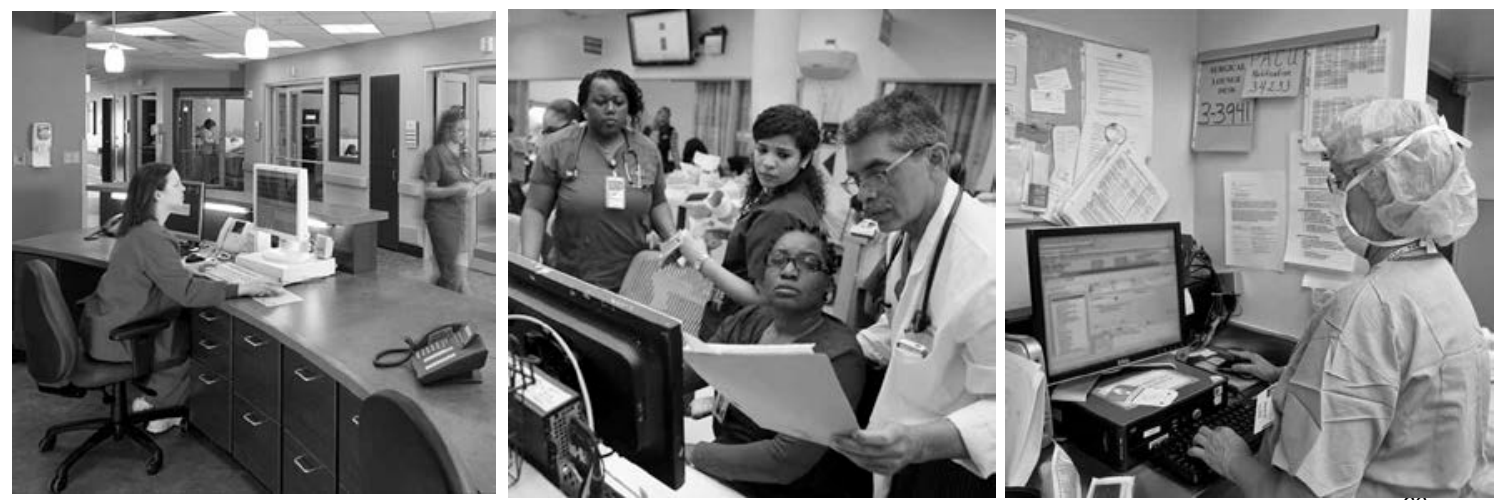

Nurses using an EHR at a central station (left, center) and in a post-anesthesia unit (right).

\subsubsection{Experience using computers}

In general, nurses are bimodal in their use of computers and EHRs. This largely depends on whether the nurse used computers during his/her education. Thus, older nurses are generally less comfortable with computers and EHRs.

Due to computer use starting in childhood, younger nurses are more likely to have experience with and be comfortable participating in computer-based training. Nurses who used computers throughout their schooling are generally more inclined to explore unfamiliar functions and perform tasks with high efficiency.

Younger nurses might also have a clearer mental model of computer-based data management, including uploading and downloading data and using computers to access other data sources. Those who did not use computers during schooling are generally less comfortable exploring computer functions and in some cases perform tasks with less efficiency.

Typing speed is not necessarily a function of age. While younger nurses might have used computers since childhood and have become proficient typists, older nurses might have taken

\footnotetext{
${ }^{59}$ Data source: 2008 National Sample Survey of Registered Nurses

${ }^{60}$ Photo sources: http://www.lecontemedicalcenter.com/upload/images/APaletz-LeConte/ICU_Hall_08.jpg, http://static01.nyt.com/images/2014/05/29/nyregion/LINCOLNweb3/LINCOLNweb3-articleLarge.jpg, http://2.bp.blogspot.com/-KIJrj9BzDEc/U6pqK0TtCel/AAAAAAAAABc/1472v8aHLUU/s1600/Jim+West+ photo+for+RN+documentation+article.jpg
} 
typing classes, which were more common in years past, and have also become efficient at data entry tasks due to their own cumulative years of interacting with computers.

\subsubsection{Sample EHR tasks by nurses}

- Enter patient medical history

- Enter vital signs (e.g., heart rate, blood pressure, oxygen saturation) into patient chart

- View patient lab and test results (e.g., cholesterol, liver function tests, x-ray)

- View patient history

- View physician's notes

- Review patient medication list

- Review pediatric patients' growth charts

- Access and print educational materials for patients

- Enter verbal/phone orders for lab tests (e.g., rapid flu test, CT scan, CBC), vaccines, and procedures on behalf of the MD

- Order referrals (e.g., for specialists, ancillary care) on behalf of the MD.

- Print, fax, or electronically send orders to other healthcare facilities

- Barcode scan medications and document administration

- Document a patient's physical assessment

- Document pain scores and interventions

- Document patient intake and output (e.g., intravenous fluid volumes, fluid taken orally, urinary output)

- Document care, such as administering a vaccine or providing patient education

- Document nursing plan of care

- Document patient communication (e.g., a patient phone call)

- Schedule appointments

- Add new patient information into the system

- Review physicians' schedules 
- Perform patient admission assessments

- Perform patient discharge and transfer assessments

- Communicate with patients via secure email regarding follow-up inquiries

- Communicate with office staff and other clinicians

\subsubsection{Performance-shaping factors}

The following factors, related to occupational responsibility, training, and familiarity with computers, could be expected to influence nurses' interactions with EHRs:

- Fast-paced environments. Working in fast-paced work environments can create stress and fatigue, leading users to seek shortcuts and workarounds to complete required tasks in time available

- Little time between patients. Lacking time between patients can make nurses more likely to delay entering data into the EHR. This would require them to rely more on memory to correctly recall and associate information and observations with the correct patient

- Working with multiple patients simultaneously. This requires nurses to rely more on memory to correctly recall and associate information and observations with the correct patient

- Need for clinician input. Confirming details or consulting with clinicians with intensive schedules might delay work

- Alternating communication modes. Switching frequently between phone-based, paperbased, EHR-based, email, and in-person tasks and communication modes

- Excessive alerts and pop-ups. Encountering an extensive number of safety alerts and pop-ups might drive users to seek workarounds and ignore pop-ups

- Developing alarm fatigue. Developing alarm fatigue can lead to ignored or missed audible alarms, as well as a disdain for on-screen warnings

- Changing documentation modes. Shifting from documenting in paper charts to using a software-based solution might challenge familiar methods of documentation

- Lacking reference material. Lacking readily available reference material (e.g., user manual, quick reference guide) can lead the nurse to rely on memory to recall operating procedures

- Interaction effect. Wearing protective equipment such as gloves might inhibit movement, visibility, and typing 


\subsection{Advanced Practice Registered Nurse}

\subsubsection{Occupational description}

Advance Practice Registered Nurses (APRN) increase access to health care by providing primary and secondary care services. APRN are registered nurses with additional graduate level education and national certification. There are four main APRN roles:

- Nurse Practitioners (NP) - serve in primary and specialty care settings within the hospital and outpatient care

- $\quad$ Nurse Anesthetists (CRNA) - primarily work in hospitals providing care and delivery of anesthesia for surgical, diagnostic and therapeutic procedures (see User Profile \#10, Anesthesia Care Providers)

- $\quad$ Nurse-midwives (CNM) - provide primary care for a wide range of women's health services (e.g., gynecological, prenatal, labor and delivery, postpartum)

- Clinical Nurse Specialists (CNS) - provide patient care and expertise in a specialty area based on a patient population, care setting, or disease process (e.g., diabetes, wounds)

The scope of practice for APRNs varies by state and specialty, and is determined by individual state laws and regulations. Many APRN can diagnose and treat acute, episodic, or chronic illnesses, as part of a healthcare team and increasingly, independently, depending on the state. Nurse practitioners may prescribe medicine as well as order, perform, or interpret diagnostic tests, independently or under the supervision of a physician depending on the state. ${ }^{61}$ We will focus primarily on NPs throughout this paper.

An APRN's occupational responsibility often depends on the work site and patient population. APRNs may work under physician supervision, in collaboration with a team of physicians and other healthcare professionals, but an increasing number of states allow APRNs to work autonomously. The table below shows a breakdown of specialties for NPs involved in patient care. $^{62}$

\begin{tabular}{lr} 
Practice Specialty for NPs $^{63}$ & $\%$ \\
\hline Primary Care & 48 \\
Other & 20 \\
Internal Medicine Subspecialties & 13 \\
Surgical Specialties & 9 \\
Psychiatry/Mental Health & 6 \\
Pediatric Subspecialties & 3
\end{tabular}

${ }^{61}$ Adapted from text found at http://www.bls.gov/oes/current/oes291171.htm

${ }^{62}$ Adapted from text found at 2012 National Sample Survey of Nurse Practitioners

${ }^{63}$ Data source: 2012 National Sample Survey of Nurse Practitioners 
No Specialty

\subsubsection{Demographics (USA)}

- Age: 48 years on average for NPs ${ }^{64}$

- Sex: Approximately $93 \%$ of NPs are female ${ }^{65}$

- Population: As of 2014 , there are around $205,000 \mathrm{NPs}^{66}$

\subsubsection{Education}

All APRNs have additional education and clinical training beyond their training as a Registered Nurse. An APRN must earn a bachelor's degree in nursing, a registered nurse license, a graduate nursing degree (either a masters in nursing or a doctor of nursing practice degree), national board certification, and state licensure and registration. ${ }^{67}$

\subsubsection{Licensure}

After achieving board certification by passing a national examination, APRNs can earn additional credentials at the state and federal level. ${ }^{68}$ All APRNs must complete continuing education (CE) and/or pass recertification exams throughout their career, as well as fulfill minimum practice time requirements, to maintain an APRN license. CE requirements vary by state and professional organization but share the goal of ensuring APRNs' knowledge and skills remain abreast of modern technological advances and medical discoveries.

\subsubsection{Electronic Health Record (EHR) Use environment(s)}

APRNs work and use EHRs in a wide variety of environments, including inpatient and outpatient hospital units, retail-based clinics, federal clinics, long-term care facilities, private physician or NP offices, home healthcare service organizations, nursing care facilities, schools, correctional facilities, ${ }^{69}$ and military healthcare ${ }^{70}$ settings.

The following table provides the distribution of NPs working in various environments. Note that NPs work in many more settings than those listed and some work in multiple settings.

\begin{tabular}{lr} 
Primary Work Settings $^{71}$ & $\%$ \\
\hline Ambulatory & 57 \\
Hospital & 31 \\
Long-Term and Elder Care & 5
\end{tabular}

642012 National Sample Survey of Nurse Practitioners

${ }^{65} 2012$ National Sample Survey of Nurse Practitioners

${ }^{66}$ AANP National NP Database, 2014

${ }_{67}^{67}$ Adapted from text found at http://www.aanp.org/images/about-nps/npgraphic.pdf

${ }^{68}$ Adapted from text found at http://www.nursepractitionerschools.com/faq/np-vs-physician-assistant

${ }^{69}$ Adapted from text found at

http://bhpr.hrsa.gov/healthworkforce/supplydemand/nursing/nursepractitionersurvey/npsurveyhighlights.pdf

${ }^{70} \mathrm{http}: / / \mathrm{www}$.airforce.com/careers/detail/nurse-practitioner/

${ }^{71}$ Data source: 2012 National Sample Survey of Nurse Practitioners 
Other Setting

Public or Community Health

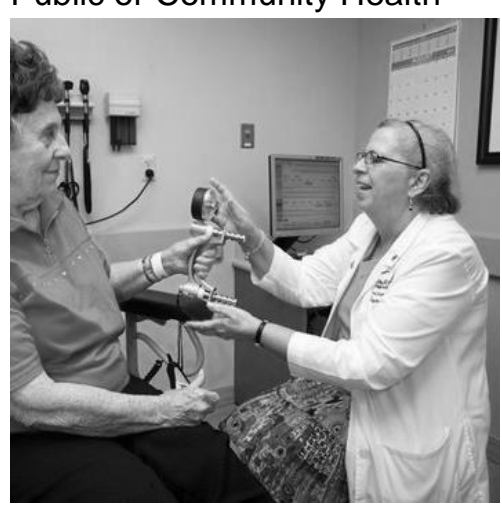

Advanced Practice Nurses interacting with EHRs.
5

2
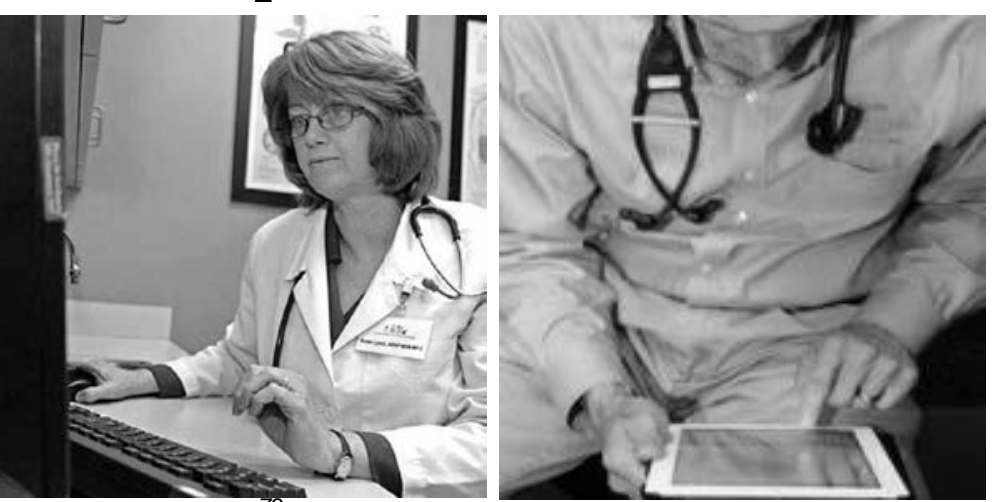

\subsubsection{Experience using computers}

In general, APRNs are bimodal in their use of computers and EHRs. This largely depends on whether the APRN used computers during his/her education. Thus, older APRNs are generally less comfortable with computers and EHRs.

Due to computer use starting in childhood, younger APRNs are more likely to have experience with and be comfortable participating in computer-based training. APRNs who used computers throughout their schooling are generally more inclined to explore unfamiliar functions and perform tasks with high efficiency.

Younger APRNs might also have a clearer mental model of computer-based data management, including uploading and downloading data and using computers to access other data sources. Those who did not use computers during schooling are generally less comfortable exploring computer functions and in some cases perform tasks with less efficiency.

Typing speed is not necessarily a function of age. While younger APRNs might have used computers since childhood and have become proficient typists, older APRNs might have taken typing classes, which were more common in years past, and also become fast at data entry tasks due to their own cumulative years of interacting with computers.

\subsubsection{Representative EHR tasks}

- Enter, edit, and view key patient information and reminders (e.g., allergies, problems, physician notes including assessment and plan)

- Reconcile the patient's medication list

\footnotetext{
${ }^{72}$ Photo sources: http://newoldage.blogs.nytimes.com/2013/08/22/a-surgical-center-for-older-patients/?_r=0, http://www.newsworks.org/index.php/local/healthscience/79826-pa-bill-would-allow-nurse-practitioners-to-workindependent-from-doctors, http://www.usatoday.com/story/news/nation/2014/08/18/nurse-practitioner-independencelaws/13470733/
} 
- Review diverse clinical materials to obtain a picture of the patient's current status, to make diagnoses, and to initiate or modify a care plan (e.g., review pediatric patients' growth charts and prior notes to assess the effects of prior treatment(s))

- Create or update a problem list, allergies, or other patient summary information.

- Initiate patient referrals to physicians and other clinicians (e.g., consultation with a specialist or assessment/intervention by another healthcare providers such as a dietician or speech therapist)

- Prescribe and transmit prescriptions electronically (outpatient or discharge)

- Enter medication orders (inpatient)

- Enter orders for vaccines, laboratory or diagnostic tests, and procedures (inpatient or outpatient)

- Modify or discontinue orders

- Receive, view, and acknowledge a patient's lab results (e.g., cholesterol, liver function tests)

- Communicate with colleagues about patient status or care plan issues.

- View patient data and other information in a chronological order to assess care trajectory

- Access and print patient educational materials

- Order or request follow-up appointments

- Communicate securely with patients electronically regarding inquiries

\subsubsection{Performance-shaping factors}

The following factors, related to occupational responsibility, training, and familiarity with computers, are just some of those that can influence an NP's interactions with EHRs:

- Fast-paced environments. Working in fast-paced work environments can create stress and fatigue, leading users to seek shortcuts and workarounds to complete required tasks in time available

- Production Pressures. APRNs can face significant production pressure in their daily work. For example, insufficient time to see patients or between patients can make APRNs more likely to avoid seeking information, to delay entering data into the EHR or to enter less information than they would otherwise. Such behaviors could require them to rely more on memory to correctly recall and associate information and observations about a patient 
- Working with multiple patients simultaneously. This requires APRNs to rely more on memory to correctly recall and associate information and observations with the correct patient

- Need for clinician input. Confirming details or consulting with physicians with intensive schedules might delay work

- Alternating communication modes. Switching frequently between phone-based, paperbased, EHR-based, email, and in-person tasks and communication modes

- Excessive alerts and pop-ups. Encountering an extensive number of safety alerts and pop-ups might drive users to seek workarounds and ignore pop-ups

- Developing alarm fatigue. Developing alarm fatigue can lead to ignored or missed audible alarms, as well as a disdain for onscreen warnings

- Changing documentation modes. Shifting from documenting in paper charts to using a software-based solution might challenge familiar methods of documentation

- Lacking reference material. Lacking readily available reference materials can make APRNs rely on memory to recall procedures and instructions

- Interaction effect. Wearing protective equipment such as gloves can inhibit movement, visibility, and typing

- Stress of dealing with difficult patients or situations. Under stressful conditions, APRNs might be more likely to take shortcuts, miss relevant cues, or to become frustrated when presented with obstacles to achieving their clinical goals while using the EHR

- Fatigue and Sleep Deprivation. APRNs can sometimes work long hours or can be working under the effects of acute and chronic sleep loss. This can lead to inattention, haste, a lower likelihood of detecting information presented in an EHR (thus suggesting a need to make critical information more salient), and a higher likelihood of ignoring prompts

\subsection{Physician Assistant}

\subsubsection{Occupational description}

Physician Assistants (PAs) increase patient access to healthcare by providing primary and secondary care services under the direct supervision of a physician. PAs scope of practice varies by state law, facility policies, and individual education and experience. PAs typically perform a wide range of tasks delegated by a physician as part of a healthcare team including diagnose and treat acute, episodic, or chronic illnesses; prescribe medications; order, perform, or interpret test results, and even assist in the conduct of surgical procedures. Thus, PAs often function in a manner similar to nurses or even advanced practice nurses. 
A PA's occupational responsibility and title often depend on specialty, work environment and patient population served. PAs must work with physician oversight, although the extent of oversight varies by state and specialty. The following table lists the distribution of PAs across common specialties:

\begin{tabular}{lr} 
Practice Specialty for PAs & $\mathbf{\%}^{\mathbf{7 3}}$ \\
\hline Primary Care & 32 \\
Surgical Subspecialties & 27 \\
Other Specialties & 19 \\
Emergency Medicine & 11 \\
Internal Medicine Subspecialties & 10 \\
Pediatric Subspecialties & 2
\end{tabular}

\subsubsection{Demographics (USA)}

- Age: 37 years on average ${ }^{74}$

- Sex: Approximately $67 \%$ are female and $33 \%$ are male for clinically practicing PAs ${ }^{75}$

- Population: As of 2015 , there are around $91,935 \mathrm{PAs}^{76}$

\subsubsection{Education}

Before entering an accredited PA program, PAs must hold a bachelor's degree and have approximately three years of hands-on patient care experience. A typical PA program is three years long and includes classroom instruction and 2,000 hours of clinical rotations. PA students study several subjects including anatomy, physiology, physical diagnosis, pathophysiology, and medical ethics.

The PA's clinical rotations include generalist primary care in ambulatory clinics, physician offices, and long-term care facilities. In more advanced rotations, the PA might focus on one or more of the following: family medicine, internal medicine, obstetrics and gynecology, pediatrics, general surgery, emergency medicine, and psychiatry. ${ }^{77}$

\subsubsection{Licensure}

A PA program graduate earns a master's degree and is eligible to take the Physician Assistant National Certifying Exam (PANCE), which is an exam administered by the National Commission

\footnotetext{
${ }^{73}$ Note: Table taken from the 2013 AAPA Annual Survey Data Tables. Values add up to $101 \%$ which is likely due to rounding errors.

Data source: https://www.aapa.org/WorkArea/DownloadAsset. aspx?id=2902

${ }^{74} 2013$ AAPA Annual Survey Report https://www.aapa.org/WorkArea/DownloadAsset.aspx?id=2902

${ }^{75} 2013$ AAPA Annual Survey Report https://www.aapa.org/WorkArea/DownloadAsset.aspx?id=2902

${ }^{76} \mathrm{http} / / / \mathrm{kff}$.org/other/state-indicator/total-physician-assistants/

${ }^{77}$ Adapted from https://www.aapa.org/Become-a-PA/
} 
on Certification of Physician Assistants (NCCPA).$^{78}$ All PAs must pass the PANCE and meet all state-specific requirements to obtain a license to see patients as a PA.

All PAs must complete continuing medical education (CME) and take recertification exams throughout their career to maintain a PA license. CME requirements vary by state and professional organization but share the goal of ensuring that PA's knowledge and skills remain abreast of modern technological advances and medical discoveries.

\subsubsection{Electronic Health Record (EHR) Use environment(s)}

PAs work in a wide variety of environments, including inpatient and outpatient hospital units, retail-based clinics, federal clinics, long-term care facilities, private physician offices, home healthcare service organizations, nursing care facilities, schools, correctional facilities, and military healthcare settings. ${ }^{79}$ The following table provides the distribution of PAs working in various settings.

\begin{tabular}{lr} 
Primary Work Settings $^{\mathbf{8 0}}$ & $\%$ \\
\hline Physician's offices & 58 \\
Hospital & 23 \\
Outpatient care centers & 7 \\
Government & 4 \\
Educational services & 3
\end{tabular}
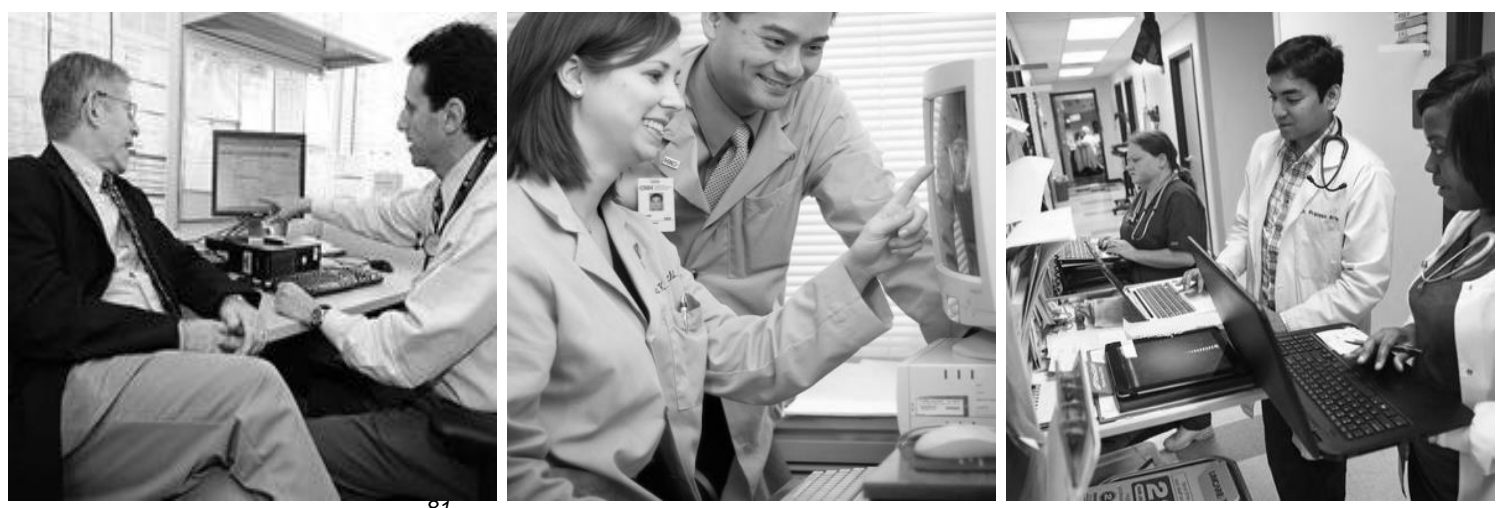

PAs interacting with EHRs. ${ }^{81}$

\subsubsection{Experience using computers}

In general, PAs are bimodal in their use of computers and EHRs. This largely depends on whether the PA used computers during his/her education. Thus, older PAs are generally less comfortable with computers and EHRs.

\footnotetext{
${ }_{78}^{78}$ Adapted from https://www.aapa.org/Become-a-PA/

${ }^{79} \mathrm{https}: / / \mathrm{www}$.aapa.org/WorkArea/DownloadAsset.aspx?id=898

${ }^{80}$ Data source: http://www.bls.gov/ooh/healthcare/physician-assistants.htm\#tab-3

${ }^{81}$ Photo sources: http://www.cepamerica.com/news-resources/perspectives-on-the-acute-care-continuum/2015-january/3barriers-(and-1-solution)-to-successful-ehr-adop, http://www.northshore.org/physicians/epic-training/, http://www.usatoday.com/story/news/nation/2015/05/25/athenahealth-epic-safety-reliability-electronic-healthrecords/27499877I
} 
Due to computer use starting in childhood, younger PAs are more likely to have experience with and be comfortable participating in computer-based training. PAs who used computers throughout their schooling are generally more inclined to explore unfamiliar functions and perform tasks with high efficiency.

Younger PAs might also have a clearer mental model of computer-based data management, including uploading and downloading data and using computers to access other data sources. Those who did not use computers during schooling are generally less comfortable exploring computer functions and in some cases perform tasks with less efficiency.

Typing speed is not necessarily a function of age. While younger PAs might have used computers since childhood and have become proficient typists, older PAs might have taken typing classes, which were more common in years past, and also become fast at data entry tasks due to their own cumulative years of interacting with computers.

\subsubsection{Sample EHR tasks}

- Enter, edit, and view key patient information and reminders (e.g., allergies, problems, physician notes including assessment and plan)

- Reconcile the patient's medication list

- Review diverse clinical materials to obtain a picture of the patient's current status, to make diagnoses, and to initiate or modify a care plan (e.g., review pediatric patients' growth charts and prior notes to assess the effects of prior treatment(s))

- Create or update a problem list, allergies, or other patient summary information

- Initiate patient referrals to other physicians and other clinicians (e.g., consultation with a specialist or assessment/intervention by another healthcare providers such as a dietician or speech therapist)

- Prescribe and transmit prescriptions electronically (outpatient or discharge)

- Enter medication orders (inpatient)

- Enter orders for vaccines, laboratory or diagnostic tests, and procedures (inpatient or outpatient)

- Modify or discontinue orders

- Receive, view, and acknowledge a patient's lab results (e.g., cholesterol, liver function tests)

- Communicate with colleagues about patient status or care plan issues.

- View patient data and other information in a chronological order to assess care trajectory 
- Access and print patient educational materials

- $\quad$ Order or request follow-up appointments

- Communicate securely with patients electronically regarding inquiries

\subsubsection{Performance-shaping factors}

The following factors, related to occupational responsibility, training, and familiarity with computers, are just some of those that can influence PA's interactions with EHRs:

- Fast-paced environments. Working in fast-paced work environments can create stress and fatigue, leading PAs to seek shortcuts and workarounds to complete required tasks in time available

- Production Pressures. PAs can face significant production pressure in their daily work. For example, insufficient time to see patients or between patients can make PAs more likely to avoid seeking information, to delay entering data into the EHR or to enter less information than they would otherwise. Such behaviors could require them to rely more on memory to correctly recall and associate information and observations about a patient

- Working with multiple patients simultaneously. This requires PAs to rely more on memory to correctly recall and associate information and observations with the correct patient

- Need for clinician input. Confirming details or consulting with physicians with intensive schedules might delay work

- Alternating communication modes. Switching frequently between phone-based, paperbased, EHR-based, email, and in-person tasks and communication modes

- Excessive alerts and pop-ups. Encountering an extensive number of safety alerts and pop-ups might drive users to seek workarounds and ignore pop-ups

- Developing alarm fatigue. Developing alarm fatigue can lead to ignored or missed audible alarms, as well as a disdain for onscreen warnings

- Changing documentation modes. Shifting from documenting in paper charts to using a software-based solution might challenge familiar methods of documentation

- Lacking reference material. Lacking readily available reference materials can make PAs rely on memory to recall procedures and instructions

- Interaction effect. Wearing protective equipment such as gloves can inhibit movement, visibility, and typing 
- Stress of dealing with difficult patients or situations. Under stressful conditions, PAs might be more likely to take shortcuts, miss relevant cues, or to become frustrated when presented with obstacles to achieving their clinical goals while using the EHR

- Fatigue and Sleep Deprivation. PAs might sometimes work long hours or can be working under the effects of acute and chronic sleep loss. This can lead to inattention, haste, a lower likelihood of detecting information presented in an EHR (thus suggesting a need to make critical information more salient), and a higher likelihood of ignoring prompts

\subsection{Anesthesia Care Providers}

\subsubsection{Occupational description}

Anesthesia care providers (ACPs) include anesthesiologists, certified registered nurse anesthetists (CRNAs), and anesthesia assistants. ACPs provide pre-, intra-, and post-operative care of surgical patients as well as patients undergoing other invasive procedures requiring anesthesia care (e.g., gastroenteroscopy, interventional cardiology and radiology, pediatric diagnostic radiology and radiation oncology). In addition to their pre- and post-operative uses of EHRs, which is similar to that of surgeons and advanced practice nurses, ACPs have a relatively unique use for EHRs in the intraoperative period. During this time, ACPs contemporaneously document all aspects of anesthesia care provided to a single patient. While similar to that of an intensive care unit nurse caring for a high-acuity patient, intraoperative anesthesia record keeping is more intensive and time-critical.

ACPs work as part of a team that includes an interventionalist physician (especially surgeons), several perioperative nurses, and other healthcare professionals. Most CRNAs and all anesthesia assistants are directed or supervised by anesthesiologists.

- Anesthesiologists are physicians with the training and expertise to provide perioperative patient care including anesthesiology, critical care, acute and chronic pain management, and resuscitation.

- There are more than 40,000 anesthesiologists in the US, representing just over $5 \%$ of all physicians. However, after excluding primary care and generalist physicians (internists and pediatricians), anesthesiology is one of the most common specialties.

\subsubsection{Demographics (USA) ${ }^{82}$}

- Age: Almost $46 \%$ of anesthesiologists are over 55 years old (as of December 2008)

- Sex: Approximately $79 \%$ of anesthesiologists are male (as of December 2008$)^{83}$ although the percentage of women entering surgery is increasing dramatically (38\% of surgical residents were female in 2009). In fact, the gender misdistribution of ACPs is much greater if one excludes obstetricians/gynecologists where $46 \%$ are female (and a

\footnotetext{
${ }^{82}$ Average age data are not available for ACPs.

${ }^{83}$ http://www.acshpri.org/documents/ACSHPRI_Surgical_Workforce_in_US_apr2010.pdf
} 
preponderance of new Ob/Gyn residents are female). Only about 5-6\% of all urologists, thoracic ACPs, and orthopedic ACPs are female.

\subsubsection{Education}

Anesthesiologists must complete four years of undergraduate school, four years of medical school, a one-year internship and 3 years of anesthesiology residency. An additional 1-2 year fellowship is needed to specialize in cardiothoracic, pediatric, neurological, or obstetric anesthesiology, critical care, or pain management. Like other physicians, anesthesiologists must complete continuing medical education (CME) throughout their career to maintain their medical license and board certification. CME requirements vary by state (to maintain licensure) and professional organizations (for maintenance of certification) but share the goal of ensuring physicians' knowledge and skills remain abreast of modern technological advances and medical discoveries. Maintenance of certification in anesthesiology requires additional training (e.g., realistic patient simulation of critical evens) and written examinations.

CRNAs must first be a licensed RN (see User Profile \#4) with critical care certification, at least one (but typically two) years of experience in critical care, successfully complete 3 years of nurse anesthesia training (leading to a MS or DNP degree), and pass a certification examination.

\subsubsection{Electronic Health Record (EHR) Use environment(s)}

ACPs work in a wide variety of procedural environments, from large academic medical centers to military surgery centers. ACPs typically work with patients on a short-term basis, due to the episodic nature of most procedures. ACPs work in office-based practices, stand-alone surgical centers, hospitals, academic medical centers, or within fully integrated healthcare organizations, such as the Veterans Health Administration. In larger group settings, ACPs benefit by being better able to communicate and coordinate patient care with other healthcare providers. ${ }^{84}$

Once a patient is scheduled for a procedure that requires anesthesia, an anesthesiologist (or sometimes an unsupervised CRNA in rural locales) reviews the patient's relevant information to ascertain the risks of receiving anesthesia, to optimize the patient prior to their procedure, and to develop a plan of care. The anesthesiologist may order additional diagnostic tests, request consultation with specialists (e.g., cardiologist, pulmonologist), and prescribe treatments prior to the procedure. During the anesthetic, the ACP documents in real-time each anesthesia treatment and patient response, and also documents (or confirms if automatically captured from the physiologic monitor) the patients' vital signs. Post-operative management has similar EHR requirements as those of surgeons or intensivists.

\footnotetext{
${ }^{84}$ Adapted from text found at https://www.facs.org/education/resources/medical-students/lifestyle
} 


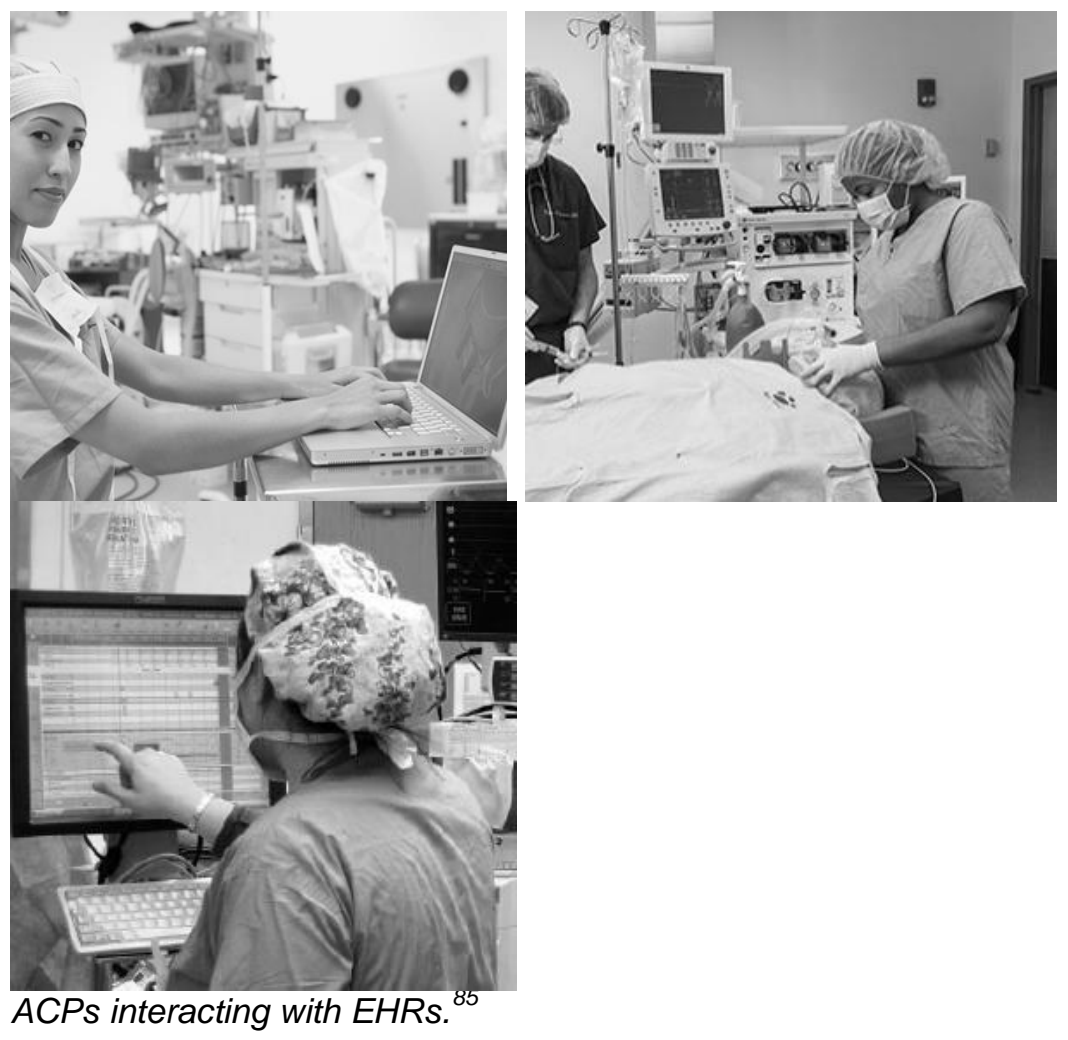

\subsubsection{Experience using computers}

ACPs routinely use cutting-edge technology on a daily basis as part of their profession, including complex anesthesia workstations, multi-parameter computer-based vital signs monitors, infusion pumps, and ultrasound machines. Thus, with the exception of a small proportion of older practitioners, ACPs are probably more comfortable using computers and technology than any other medical professional.

\subsubsection{Sample EHR tasks}

- Enter, edit, and view key patient information (e.g., vital signs), diagnostic test results, and prompts or reminders (e.g., compliance with evidence-based guidelines)

- Review a patient's medications, problem list, and prior anesthesia and procedure notes

- Review diverse clinical materials to obtain a picture of the patient's current status, to make diagnoses, and to initiate or modify a care plan

- Create a pre- or post-operative note about a patient.

\footnotetext{
${ }^{85}$ Photo sources: http://ovydizekowan.site40.net/graduate-degrees-in-nursing-informatics.php, https://www.quinnipiac.edu/academics/colleges-schools-and-departments/school-ofmedicine/academics/anesthesiologist-assistant-program/, http://www.asahq.org/resources/publications/newsletterarticles/2015/may-2015/electronic-health-records
} 
- Initiate patient referrals to other clinicians (e.g., consultation with a specialist or assessment/intervention by another healthcare providers such as a dietician or speech therapist)

- Prescribe and transmit prescriptions electronically (inpatient or outpatient)

- Enter, modify or discontinue medication orders (inpatient or outpatient)

- Enter orders for laboratory or diagnostic tests, and procedures (inpatient or outpatient)

- Communicate with colleagues about patient status or care plan issues.

- View patient data and other information in a chronological order to assess care trajectory

- Access and print patient educational materials

- Communicate securely with patients electronically regarding inquiries

\subsubsection{Performance-shaping factors}

The following factors, related to occupational responsibility, training, and familiarity with computers, could be expected to influence physicians' interactions with EHRs:

- Fast-paced environments. Working in fast-paced work environments can create stress and fatigue, leading ACPs to seek shortcuts and workarounds to complete required tasks in the time available

- Production Pressures. Most ACPs face significant production pressure in their daily work. For example, they feel that they have insufficient time to see patients preoperatively or to complete documentation at the end of a procedure before moving on to the next patient. These pressures can make the ACP more likely to avoid seeking information, to delay entering data into the EHR, or to enter less information than they would otherwise. Such behaviors could require ACPs to rely more on memory to correctly recall and associate information and observations about a patient

- Working with multiple patients simultaneously. When supervising the care of multiple patients by CRNAs or AAs, the anesthesiologist might need to rely more on memory to correctly recall and associate information and observations with the correct patient

- Need for clinician input. Confirming details or consulting with other clinicians with intensive schedules might delay cases and increase production pressure

- Alternating communication modes. Switching frequently between phone-based, paperbased, EHR-based, email, and in-person tasks and communication modes

- Excessive alerts and pop-ups. Encountering an extensive number of safety alerts and pop-ups might drive users to seek workarounds and ignore pop-ups 
- Changing documentation modes. Shifting from documenting in paper charts to using a software-based solution might challenge familiar methods of documentation

- Lacking reference material. Lacking readily available reference material can make ACPs rely on memory to recall procedures and instructions

- Interaction effect. Routinely wearing and changing gloves can impair typing or touching of the user interface. The need to wear additional personal protective gear (e.g., in patients with antibiotic resistant infections, tuberculosis, etc.) can further inhibit movement and visibility

- Stress of dealing with difficult patients or situations. Under stressful conditions, ACPs might be more likely to take shortcuts, miss relevant cues, or to become frustrated when presented with obstacles to achieving their clinical goals while using the EHR

- Fatigue and Sleep Deprivation. ACPs might work extended hours and can be under the effects of acute and chronic sleep loss. This can lead to inattention, haste, a lower likelihood of detecting information presented in an EHR (thus suggesting a need to make critical information more salient), and a higher likelihood of ignoring prompts

\subsection{Physician}

\subsubsection{Occupational description}

A physician's occupational responsibility is based on his/her specialty, the environment in which s/he works, and the patients s/he serves. Physicians typically work as part of a team that includes additional physicians, nurses, and other healthcare specialists. In some cases, physicians oversee the work of advanced practice nurses, physician assistants, registered nurses, licensed practical nurses, nursing assistants, and home health aides.

A physician's work might focus on one or more of the following specific fields:

- A health condition, such as diabetes

- A body part, such as the skin

- A group of people, such as the elderly

- A workplace, such as an emergency department in a hospital

Physicians commonly perform the following tasks to diagnose and treat injuries or illness, and to maintain patient health and well-being:

- Examine patients for abnormalities

- Review medical histories

- Make clinical assessments and diagnoses 
- Order, perform, and interpret diagnostic tests

- Prescribe medications and other therapeutic interventions

- $\quad$ Create and modify care plans

- Communicate with other physicians, other members of the healthcare team

- Communicate with the patient and their family/caregivers about the care plan

- Counsel patients on preventative healthcare, including diet and hygiene

\subsubsection{Demographics (USA)}

- Age: $50 \%$ of physicians are between $40-59$ years old ${ }^{86}$

- Sex: Approximately $70 \%$ are male and $29 \%{ }^{87}$ are female ${ }^{88}$

- Population: As of 2010 , there were 850,085 physicians $^{89}$

\subsubsection{Education}

A physician must complete four years of undergraduate school, four years of medical school, a one year clinical internship and between two and seven years of advanced clinical training, residency and/or fellowship, depending on the physician's intended specialization. Aspiring physicians study a wide breadth of topics, including basic sciences such as anatomy and pharmacology, population health, and a broad range of clinical topics from gynecology to medical ethics. Throughout medical school, medical students care for patients in both inpatient and outpatient settings with progressive responsibility under the supervision of experienced physicians. In their last year or two, they might begin to specialize in a particular area of medicine. After passing licensure board exams and obtaining licensure from the state medical board, physicians are fully licensed to practice in that state. There is no reciprocity between states although it is typically not too difficult for a physician in one state to obtain a license in another. While a specialist physician does not require an additional license, states and individual hospitals might require specialty board certification. This requires additional years of clinical training, and then passing certification examination(s). Thus, the typical physician does not start independent clinical practice until after age 30.

In the USA, $67 \%$ of physicians are allopathic physicians (i.e., medical doctors or M.D.s), $7 \%$ are osteopathic physicians (doctors of osteopathic medicine or D.O.s), and the remaining $26 \%$ are international medical school graduates (IMGs) who might have a variety of degrees. To practice in the United States, all IMGs, regardless of specialty, must pass a comprehensive examination process and complete at least one year of additional clinical training in a US residency program.

${ }^{86} 2010$ A Census of Actively Licensed Physicians in the United States

${ }^{87}$ Note: The Federation of State Medical Boards 2010 physician census gathered data related to the ratio of male to female physicians in the USA. The sum of the values (i.e., $70 \%$ and $29 \%$ ) is $99 \%$, which is most likely due to rounding errors or non-responses.

882010 A Census of Actively Licensed Physicians in the United States

${ }^{89} 2010$ A Census of Actively Licensed Physicians in the United States 
IMGs with prior specialist training (e.g., cardiac surgeon) must repeat most of the same training in the US to be able to practice that specialty.

Although M.D.s and D.O.s are subject to similar educational requirements, D.O.s tend to approach patient care more holistically. Approximately $60 \%$ of D.O.s are practice in primary care specialties (PCPs). ${ }^{90}$

All physicians must complete continuing medical education (CME) throughout their career, as well as fulfill minimum practice time requirements, to maintain a medical license. CME requirements vary by state (to maintain licensure) and professional organizations (for maintenance of board certification) but share the goal of ensuring physicians' knowledge and skills remain abreast of modern technological advances and medical discoveries. Maintenance of certification requirements might also include additional training and even examinations.

\subsubsection{Electronic Health Record (EHR) Use environment(s)}

Physicians work in a variety of environments, including hospitals, clinics, offices, home healthcare organizations, nursing care facilities, schools, correctional facilities, and military healthcare settings.

As of $2012,60 \%$ of doctors less than 40 years of age practiced medicine in a group setting with other healthcare providers, such as in a hospital. ${ }^{91}$ Other group practice environments include specialty practices and clinics. Group settings enable physicians to collaborate with other providers and coordinate patient care. ${ }^{92}$ Increasingly group practices are multidisciplinary and might include advanced practice nurses and other healthcare workers. This is important for EHR use because there might be even more interchange of diverse personnel providing care to individual patients in these integrated group practices.

Of the nearly 1 billion annual visits to physicians' offices in 2010, more than 55\% were to primary care physicians. ${ }^{93}$ While there has been a strong movement for physicians to specialize, many 'specialists' provide their patients with some primary care services and about one-third of all US physicians identify themselves as being primary care physicians. ${ }^{94}$ Primary care physicians are trained in General Internal Medicine ( $45 \%$ of primary care practicing physicians), Family Medicine (42\%), Pediatrics (24\%) or are general practitioners (i.e., no board certification, 6\%). Most geriatricians and internal medicine/pediatrics practitioners as well as some gynecologists provide primary care services for their patients.

While the exact numbers vary depending on the source of the data and the definition of an "active" physician, the following table provides a reasonable estimate of the distribution of physicians working in various specialties. This table excludes primary care oriented disciplines.

\footnotetext{
${ }^{90}$ Adapted from text found at http://www.osteopathic.org/osteopathic-health/about-dos/what-is-a-do/Pages/default.aspx

${ }^{91} \mathrm{http}: / / \mathrm{www}$.healthecareers.com/article/most-popular-practice-setting-for-physician-employment/173573

${ }_{92}$ Adapted from text found at http://www.bls.gov/ooh/healthcare/physicians-and-surgeons.htm

${ }^{93}$ Source: National Ambulatory Medical Care Survey: 2010 Summary Tables, Accessed at:

http://www.cdc.gov/nchs/fastats/physician-visits.htm

${ }^{94}$ Data source: Agency for Healthcare Research and Quality, "The Number of Practicing Primary Care Physicians in the United States' Publication \#12-P001-2-EF, 2010,

http://www.ahrq.gov/research/findings/factsheets/primary/pcwork1/index.html
} 
Some medical and surgical specialties that deal with similar patient populations have been combined because their practice and information needs relevant to EHR design are similar.

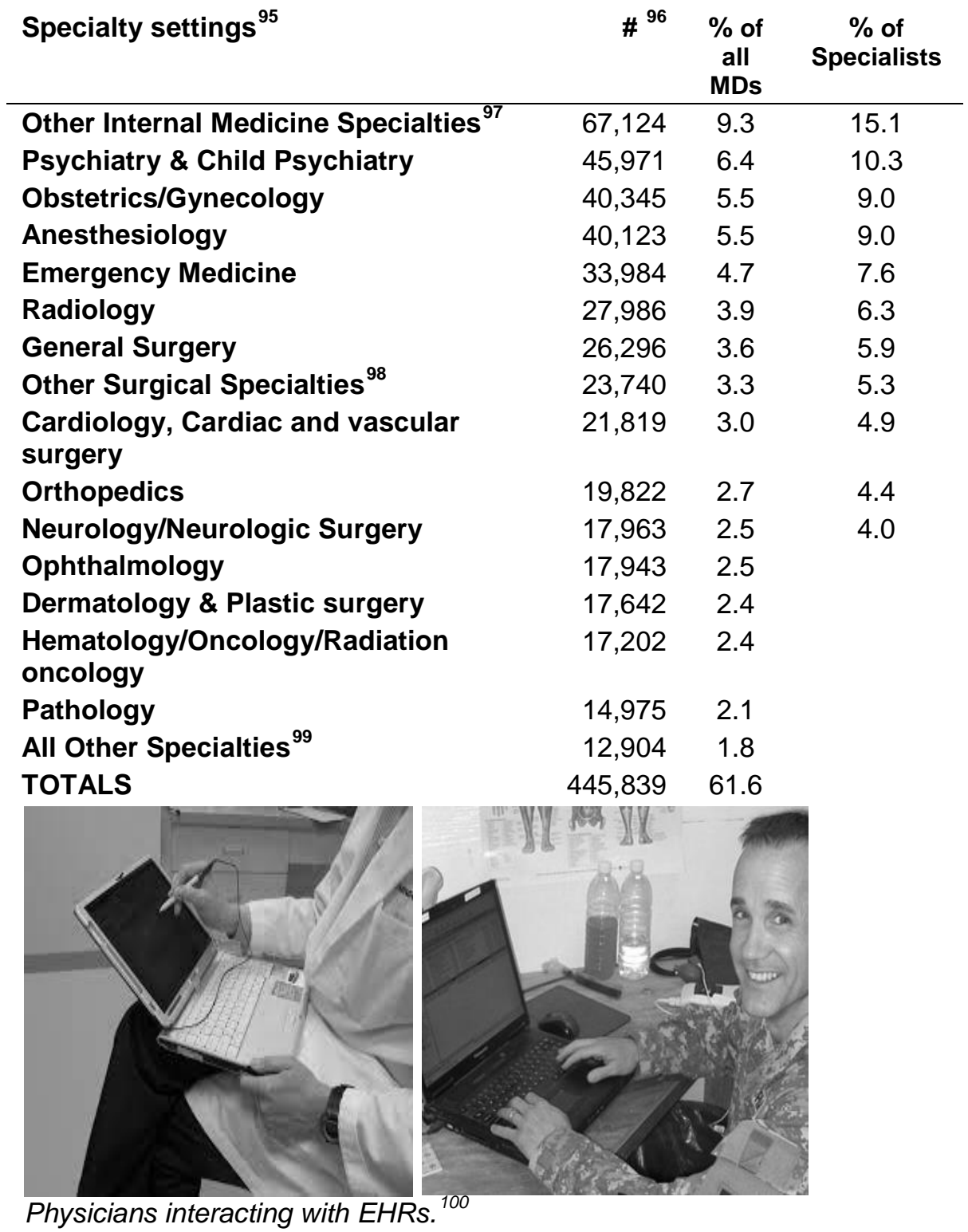

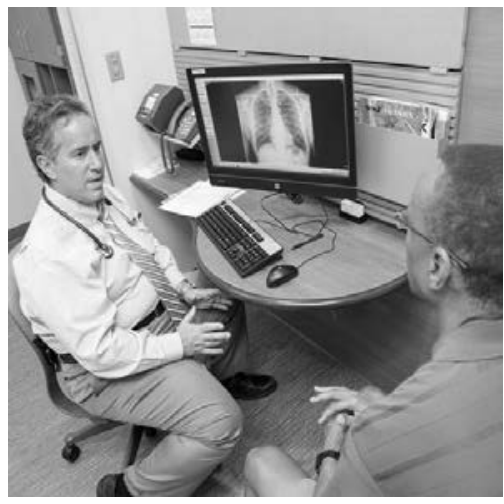

\footnotetext{
${ }^{95}$ This is 2015 data from a different data source: Special data request on State Licensing Information from Redi-Data Inc. March 2015. http://kff.org/other/state-indicator/physicians-by-specialty-area/

${ }^{96}$ Association of American Medical Colleges (AAMC). 2012 Physician Specialty Data Book, Table 1, from AMA physician master file. https://www.aamc.org/download/313228/data/2012physicianspecialtydatabook.pdf

${ }^{97}$ Other internal medicine specialties include gastroenterology, pulmonary/critical care, nephrology, infectious disease, preventive Medicine, endocrinology/diabetes/metabolism, rheumatology, and allergy/immunology.

${ }_{98}$ Other surgical specialties include (ordered by number of physicians): urology, otolaryngology and thoracic surgery.

${ }^{99}$ All other specialties above include, but are not limited to: Physical medicine \& rehabilitation (PMR) and Neonatology/perinatal medicine.

${ }^{100}$ Photo sources: http://www.wsj.com/articles/ama-urges-overhaul-of-electronic-records-1410840063, http://blog.M.D.sofkansas.com/trusting-voice-recognition-and-the-ehr/,

http://www.mc4.army.mil/story/Battalion_Surgeon_Readies_Unit_with_EMR_Garrison_Use_Exercise_Play
} 


\subsubsection{Experience using computers}

In general, while most physicians are quite comfortable with computers and EHRs, some are less comfortable and this might be somewhat related to how much the physician used computers during his/her education and training (and thus correlated with physician age).

Due to computer use starting in childhood, younger physicians are more likely to have experience with and be comfortable participating in computer-based training. Physicians who used computers throughout their schooling are generally more inclined to explore unfamiliar functions and perform tasks with high efficiency.

Younger physicians might have a clearer mental model of computer-based data management, including uploading and downloading data and using computers to access other data sources. Those physicians who did not use computers during schooling are generally less comfortable exploring computer functions and in some cases might perform computer-based tasks less efficiently than younger physicians.

\subsubsection{Representative EHR tasks}

- Enter, edit, and view key patient information and reminders (e.g., allergies, problems, physician notes including assessment and plan)

- Reconcile the patient's medication list

- Review diverse clinical materials to obtain a picture of the patient's current status, to make diagnoses, and to initiate or modify a care plan (e.g., review pediatric patients' growth charts and prior notes to assess the effects of prior treatment(s))

- Create or update a problem list, allergies, or other patient summary information.

- Initiate patient referrals to other clinicians (e.g., consultation with a specialist or assessment/intervention by another healthcare providers such as a dietician or speech therapist)

- Prescribe and transmit prescriptions electronically (outpatient or discharge)

- Enter medication orders (inpatient)

- Enter orders for vaccines, laboratory or diagnostic tests, and procedures (inpatient or outpatient)

- Modify or discontinue orders

- Receive, view, and acknowledge a patient's lab results (e.g., cholesterol, liver function tests)

- Communicate with colleagues about patient status or care plan issues. 
- View patient data and other information in a chronological order to assess care trajectory

- Access and print patient educational materials

- Order or request follow-up appointments

- Communicate securely with patients electronically regarding inquiries

\subsubsection{Performance-shaping factors}

The following factors, related to occupational responsibility, training, and familiarity with computers, are just some of those that can influence physicians' interactions with EHRs:

- Fast-paced environments. Working in fast-paced work environments can create stress and fatigue, leading users to seek shortcuts and workarounds to complete required tasks in time available

- Production Pressures. Most physicians face significant production pressure in their daily work. For example, insufficient time to see patients or between patients can make physicians more likely to avoid seeking information, to delay entering data into the EHR or to enter less information than they would otherwise. Such behaviors could require them to rely more on memory to correctly recall and associate information and observations about a patient

- Working with multiple patients simultaneously. This requires physicians to rely more on memory to correctly recall and associate information and observations with the correct patient

- Need for clinician input. Confirming details or consulting with other clinicians with intensive schedules might delay work

- Alternating communication modes. Switching frequently between phone-based, paperbased, EHR-based, email, and in-person tasks and communication modes

- Excessive alerts and pop-ups. Encountering an extensive number of safety alerts and pop-ups might drive users to seek workarounds and ignore pop-ups

- Developing alarm fatigue. Developing alarm fatigue can lead to ignored or missed audible alarms, as well as a disdain for onscreen warnings

- Changing documentation modes. Shifting from documenting in paper charts to using a software-based solution might challenge familiar methods of documentation

- Lacking reference material. Lacking readily available reference material can make physicians rely on memory to recall procedures and instructions

- Interaction effect. Wearing protective equipment such as gloves can inhibit movement, visibility, and typing 
- Stress of dealing with difficult patients or situations. Under stressful conditions, physicians might be more likely to take shortcuts, miss relevant cues, or to become frustrated when presented might obstacles to achieving their clinical goals while using the EHR

- Fatigue and Sleep Deprivation. Physicians often work long hours and can be working under the effects of acute and chronic sleep loss. This can lead to inattention, haste, a lower likelihood of detecting information presented in an EHR (thus suggesting a need to make critical information more salient), and a higher likelihood of ignoring prompts

\subsection{Surgeon}

\subsubsection{Occupational description}

A surgeon's occupational responsibilities vary based on the surgeon's clinical setting and field of expertise. Surgeons typically work as part of a team that includes other physicians (especially anesthesiologists), nurses, and other healthcare professionals. Surgeons often oversee the work of fellows, residents, interns, nurse practitioners, physician assistants, registered nurses, licensed practical nurses. In a relatively low proportion of practices, they might also oversee the work of nursing assistants, and home health aides. ${ }^{101}$

Surgeons are physicians with the training and expertise to perform surgical procedures (i.e., operations) as well as preoperative diagnosis and postoperative patient care. Surgeons might focus on general surgery or specialize. General surgery traditionally includes colorectal surgery and trauma/burn surgery even though those areas are now subspecialties.

Obstetrics/Gynecology (Ob/Gyn) is both a surgical and a non-surgical specialty but is included here for completeness. When including Ob/Gyn, surgeons represent just less than $20 \%$ of all physicians. While the exact numbers vary depending on the source of the data and the definition of an "active" physician, the following table provides a rough estimate of the distribution of surgeons working in various specialties.

\begin{tabular}{|c|c|c|}
\hline Surgical specialties ${ }^{102}$ & \# & $\begin{array}{c}\% \text { of all } \\
\text { Surgeons }\end{array}$ \\
\hline Obstetrics/Gynecology & 40,826 & 28 \\
\hline General surgery & 25,187 & 17 \\
\hline Orthopedic surgery & 19,374 & 13 \\
\hline Ophthalmology & 18,317 & 13 \\
\hline Urology & 9,771 & 7 \\
\hline Otolaryngology & 9,320 & 6 \\
\hline Plastic surgery & 6,942 & 5 \\
\hline Neurologic Surgery & 5,171 & 4 \\
\hline Thoracic surgery & 4,521 & 3 \\
\hline Vascular surgery & 3,130 & 2 \\
\hline Pediatric surgery (estimate) ${ }^{103}$ & 2,700 & 2 \\
\hline
\end{tabular}




\subsubsection{Demographics (USA) ${ }^{104}$}

- Age: Almost $46 \%$ of surgeons are over 55 years old (as of December 2008)

- Sex: Approximately $79 \%$ of surgeons are male (as of December 2008) ${ }^{105}$ although the percentage of women entering surgery is increasing dramatically (38\% of surgical residents were female in 2009). In fact, the gender misdistribution of surgeons is much greater if one excludes obstetricians/gynecologists where $46 \%$ are female (and a preponderance of new Ob/Gyn residents are female). Only about 5-6\% of all urologists, thoracic surgeons, and orthopedic surgeons are female.

\subsubsection{Education}

Surgeons must complete four years of undergraduate school, four years of medical school, a one-year internship and between 2 and 9 years of residency and/or fellowship, depending on their specialty. Like other physicians (see that User Profile \#8 for more information), aspiring surgeons study a broad range of topics, but with greater emphasis on anatomy and pathology after medical school. Surgeons can practice independently after earning their medical degree, passing their licensure exams, completing residency and fellowship training, and meeting the requirements for specialty (and subspecialty as appropriate) board certification.

Like other physicians, surgeons must complete continuing medical education (CME) throughout their career, as well as fulfill minimum practice time requirements, to maintain their medical license and board certification. CME requirements vary by state (to maintain licensure) and professional organizations (for maintenance of certification) but share the goal of ensuring surgeons' knowledge and skills remain abreast of modern technological advances and medical discoveries. Maintenance of certification requirements might also include additional training and even examinations.

\subsubsection{Practice Attributes}

Surgical care includes preoperative assessment (to determine the need for and then to schedule surgery), preoperative preparation, intraoperative surgical care, immediate post-operative management, and longer-term follow up. Surgeons obtain referrals from other physicians (e.g., internists, primary care physicians) and also obtain consultations from various specialists (anesthesiologists, pain physicians, internal medicine specialists such as cardiologists).

\subsubsection{Electronic Health Record (EHR) Use environment(s)}

Surgeons work in a wide variety of environments, from large academic medical centers to military surgery centers. Surgeons typically work with patients on a short-term basis, due to the episodic nature of most surgeries. Surgeons work in office-based practices, stand-alone surgical centers, hospitals, academic medical centers, or within fully integrated healthcare organizations such as

\footnotetext{
${ }^{104}$ Average age data are not available for surgeons.

${ }^{105} \mathrm{http}: / /$ www.acshpri.org/documents/ACSHPRI_Surgical_Workforce_in_US_apr2010.pdf
} 
the Veterans Administration or Kaiser Permanente. In larger group settings, surgeons benefit by being better able to communicate and coordinate patient care with other healthcare providers. ${ }^{106}$

Often, healthcare professionals refer the patient and send the patient's relevant information to the surgeon. Surgeons have somewhat more specialized interactions with EHR systems than do other physicians such as internists. Because many surgeons dictate their notes, especially their operative summaries, their EHR expectations might be different from other healthcare professionals (e.g., more surgeons might use voice recognition products to enter notes into an EHR).

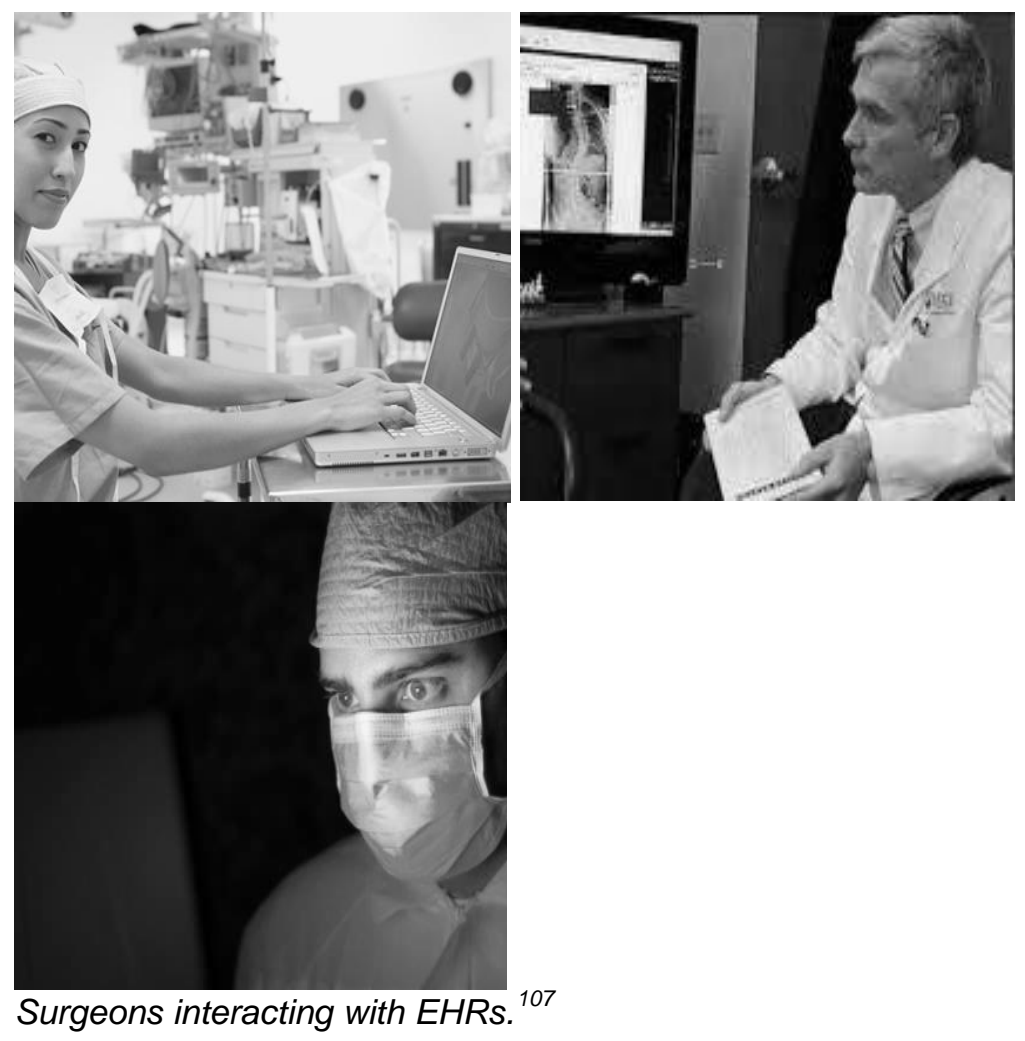

\subsubsection{Experience using computers}

Many surgeons use cutting-edge and emerging technology as part of their profession, including surgical robots. That said, surgeons are somewhat bimodal in their use of computers and EHRs older surgeons who did not use computers during their education might be more reluctant to use and less comfortable using EHRs.

Younger surgeons are more likely to have experience with and be comfortable participating in computer-based training. Surgeons who used computers throughout their schooling are generally more inclined to explore unfamiliar functions and perform tasks with high efficiency. These

\footnotetext{
${ }^{106}$ Adapted from text found at https://www.facs.org/education/resources/medical-students/lifestyle ${ }^{107}$ Photo sources: http://www.fdbhealth.com/blog/2013/december/will-meaningful-use-of-ehrs-prove-beneficial/, http://www.informationweek.com/healthcare/electronic-health-records/surgeon-cuts-vendors-out-of-ehr-quest/d/did/1252732, http://www.jimenezconsulting.com/solutions/ehr/default.aspx
} 
younger surgeons might also have a clearer mental model of computer-based data management, including uploading and downloading data and using computers to access other data sources.

\subsubsection{Representative EHR tasks}

- Enter, edit, and view key patient information and reminders (e.g., allergies, problems, physician notes including assessment and plan)

- Reconcile the patient's medication list

- Review diverse clinical materials to obtain a picture of the patient's current status, to make diagnoses, and to initiate or modify a care plan (e.g., review pediatric patients' growth charts and prior notes to assess the effects of prior treatment)

- Dictate or write operative notes

- Create or update a problem list, allergies, or other patient summary information.

- Initiate patient referrals to other clinicians (e.g., consultation with a specialist or assessment/intervention by another healthcare providers such as a dietician or speech therapist)

- Prescribe and transmit prescriptions electronically (outpatient or discharge)

- Enter medication orders (inpatient)

- Enter orders for vaccines, laboratory or diagnostic tests, and procedures (inpatient or outpatient)

- Modify or discontinue orders

- Receive, view, and acknowledge a patient's lab results (e.g., cholesterol, liver function tests)

- Communicate with colleagues about patient status or care plan issues.

- View patient data and other information in a chronological order to assess care trajectory

- Access and print patient educational materials

- Order or request follow-up appointments

- Communicate securely with patients electronically regarding inquiries

\subsubsection{Performance-shaping factors}

The following factors, related to occupational responsibility, training, and familiarity with computers, are just some of those that can influence surgeon's interactions with EHRs: 
- Fast-paced environments. Working in fast-paced work environments can create stress and fatigue, leading surgeons to seek shortcuts and workarounds to complete required tasks in time available

- Production Pressures. Most surgeons face significant production pressure in their daily work. For example, insufficient to see patients or between patients can make surgeons more likely to avoid seeking information, to delay entering data into the EHR or to enter less information than they would otherwise. Such behaviors could require them to rely more on memory to correctly recall and associate information and observations about a patient

- Working with multiple patients simultaneously. This requires surgeons to rely more on memory to correctly recall and associate information and observations with the correct patient

- Need for clinician input. Confirming details or consulting with other clinicians with intensive schedules might delay work

- Alternating communication modes. Switching frequently between phone-based, paperbased, EHR-based, email, and in-person tasks and communication modes

- Excessive alerts and pop-ups. Encountering an extensive number of safety alerts and pop-ups might drive users to seek workarounds and ignore pop-ups

- Changing documentation modes. Shifting from documenting in paper charts to using a software-based solution might challenge familiar methods of documentation

- Lacking reference material. Lacking readily available reference material can make surgeons rely on memory to recall procedures and instructions

- Interaction effect. Wearing protective equipment such as gloves can inhibit movement, visibility, and typing

- Stress of dealing with difficult patients or situations. Under stressful conditions, surgeons might be more likely to take shortcuts, miss relevant cues, or to become frustrated when presented with obstacles to achieving their clinical goals while using the EHR

- Fatigue and Sleep Deprivation. Surgeons work long hours and often work under the effects of acute and chronic sleep loss. This can lead to inattention, haste, a lower likelihood of detecting information presented in an EHR (thus suggesting a need to make critical information more salient), and a higher likelihood of ignoring prompts 


\subsection{Pharmacist}

\subsubsection{Occupational description}

Pharmacists distribute prescription medications and advise patients on how to safely and effectively use prescription medications in both the inpatient and outpatient settings. Inpatient pharmacists are part of a healthcare team where they are responsible for medication management including supply chain management. Pharmacists interact with electronic health records (EHRs) in pharmacies and other clinical settings. They might also administer immunizations, help patients manage chronic conditions, and review medication lists. Pharmacists often oversee the work of pharmacy technicians and provide consultation to physicians, nurses, APRNs, PAs, and other healthcare professionals.

\subsubsection{Demographics (USA)}

- Age: Average in the USA is 43 years (as of 2014) (108 $^{108}$

- Sex: Approximately $56 \%$ are female, $44 \%$ are male (as of 2013$)^{109}$

- Population: As of 2013 , there were 287,420 pharmacists ${ }^{110}$

\subsubsection{Education}

Becoming a pharmacist requires a Pharm.D. degree, typically earned upon the completion of four years of undergraduate school and four years of pharmacy school. Important areas of instruction during pharmacy school include: pharmaceutical chemistry, pharmacognosy, pharmacology, business management, pharmacy practice, as well as a clinical component. ${ }^{111}$ Because of the heavy use of computer-based procedures and documentation in modern pharmacy practice, pharmacists receive more informatics training than most other healthcare professionals.

Upon completion of pharmacy school, increasingly are expected to do one-year of additional clinical training (like a medical internship). Graduates can further pursue additional training with either a research focus (i.e., fellowship programs) or a clinical focus (i.e., residency programs). ${ }^{112}$

All pharmacists must complete continuing pharmacy education (CPE) throughout their career to maintain a pharmacist license. CPE requirements vary by state and board but share the goal of ensuring pharmacists' knowledge and skills remain abreast of modern advances and medical discoveries.

\subsubsection{Electronic Health Record (EHR) Use environment(s)}

Pharmacists work in variety of environments, including retail pharmacies, hospitals, clinics, mailorder pharmacies, wholesalers, and home health agencies. ${ }^{113}$ The following table provides the distribution of pharmacists working in various settings.

${ }_{108} \mathrm{http}: / /$ dpeaflcio.org/programs-publications/issue-fact-sheets/pharmacists-and-pharmacy-technicians-facts-and-figures/

${ }^{109} \mathrm{http}: / /$ dpeaflcio.org/programs-publications/issue-fact-sheets/pharmacists-and-pharmacy-technicians-facts-and-figures/

${ }_{110} \mathrm{http}$ ://dpeaflcio.org/programs-publications/issue-fact-sheets/pharmacists-and-pharmacy-technicians-facts-and-figures/

${ }_{111} \mathrm{http}$ ://www.aacp.org/resources/student/pharmacyforyou/documents/pharmd.pdf

${ }^{112}$ Adapted from text found at http://www.accp.com/stunet/compass/residency.aspx 


\begin{tabular}{lr} 
Pharmacist settings $^{114}$ & \% \\
\hline Pharmacies and drug stores & 43 \\
Hospitals; state, local, and private & 23 \\
Grocery stores & 8 \\
Department stores & 5 \\
Other general merchandise stores & 5
\end{tabular}

Clinical pharmacists can work in outpatient or inpatient settings. When in the outpatient setting they might work directly with patients but in either setting they typically oversee pharmacy technicians, and work closely with physicians and other health professionals to coordinate patient care. In these collaborative settings, pharmacists' primary goal is to ensure that the medications prescribed for patients contribute to the best possible outcomes. ${ }^{115}$ Pharmacists may also advise insurance providers or healthcare facilities, or work in the pharmaceutical industry to develop drugs. ${ }^{116}$

Clinical pharmacists are responsible for managing their facility's drug formulary (i.e., what medications will be available), ordering bulk medications for the facility, storing and organizing those medications, preparing, dispensing, and delivering the medications to the patient, assuring that dispensed medications are correctly labeled, assuring that every patient receives the correct medication, and generating accurate timely documentation of all of the above activities. Thus, clinical pharmacists, through additional training and extensive exposure to EHRs, are generally more familiar with computer use in their practice than are most physicians. Even older community pharmacists who were not exposed to computers during their training, have invariably now been forced to use EHRs in their practice for many years and become comfortable with their use.
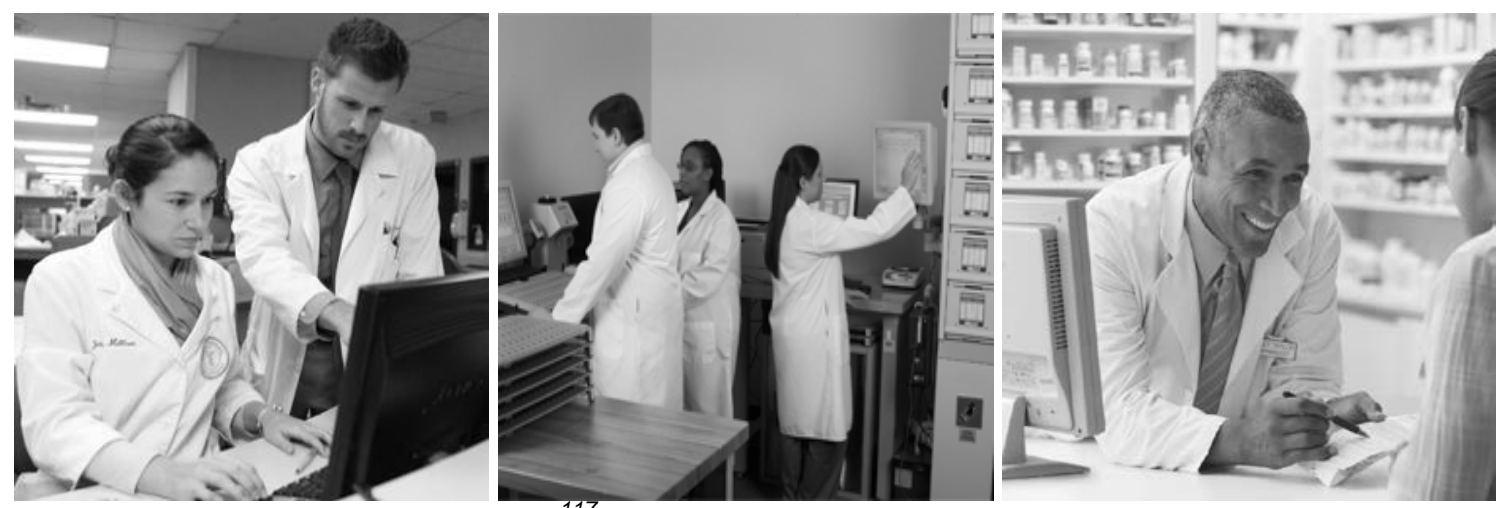

Pharmacists interacting with EHRs. ${ }^{11}$

\subsubsection{Sample EHR tasks}

- Review key patient information to determine whether the prescribed medications are meeting the patient's need and goals of care

\footnotetext{
${ }^{113} \mathrm{http} / / /$ dpeaflcio.org/programs-publications/issue-fact-sheets/pharmacists-and-pharmacy-technicians-facts-and-figures/

${ }^{114}$ Data source: http://www.bls.gov/ooh/healthcare/pharmacists.htm\#tab-3

${ }_{115}^{115}$ Adapted from text found at http://www.bls.gov/ooh/healthcare/physicians-and-surgeons.htm

${ }^{116}$ Adapted from text found at http://www.bls.gov/ooh/healthcare/pharmacists.htm\#tab-2

${ }^{117}$ Photo sources: http://www.ashpintersections.org/2015/01/new-ehr-improves-outcomes-at-the-university-of-kansashospital/, http://clinicalsolutionspharmacy.com/services/ehr_emr, http://healthit.gov/providers-professionals/pharmacyreview-and-process-q7
} 
- View patient data chronologically as flowchart showing progress over time

- Advise patients regarding prescribed medications and any potential side effects ${ }^{118}$

- Review patient prescriptions for potential drug interactions

- Receive and verify prescriptions electronically before filling

- Access and print patient educational materials

- Add new patient information into the system

- Enter patient medical history or patient assessments

- Review patient medication list

- View patient lab and test results (e.g., cholesterol, liver function tests, x-ray)

- View patient history and physician's notes

- Review pediatric patients' growth charts

- Access and print educational materials for patients

- Send notes to patients or other healthcare professionals

- Document care, such as administering a vaccine or providing patient education

- Communicate with patients via secure email

- Document patient communication (e.g., a patient phone call)

- Barcode scan medications and document administration

- Review nurses administration of medications

- Review physicians' orders on single and multiple patients

- Review pharmacy technicians' dispensing records and work

- Review, edit, document drug formularies

- Review and approve non-formulary drug requests

- Order medications (in bulk) from pharmaceutical manufacturers

- Retrieve, review, edit, and disseminate materials about medication safety and use

${ }^{118}$ Adapted from text found at http://www.uwhealth.org/quality-reports/the-pharmacists-role-on-the-patient-careteam/12224 
- Support the care team's efforts to educate patients on preventative health measures (e.g., vaccination)

- Recognize untreated health issues that could be corrected with medication therapy ${ }^{119}$

\subsubsection{Performance-shaping factors}

The following factors, related to occupational responsibility, training, and familiarity with computers, could be expected to influence pharmacists' interactions with EHRs:

- Fast-paced environments. Working in fast-paced work environments can create stress and fatigue, leading pharmacists to seek shortcuts and workarounds to complete required tasks in time available

- Production Pressures. Many pharmacists face significant production pressure in their daily work. For example, insufficient time to prepare and label medications can make pharmacists more likely to avoid seeking information, to delay entering data into the EHR or to enter less information than they would otherwise. Such behaviors could require them to rely more on memory to correctly recall and associate information and observations about a patient

- Working with multiple prescriptions simultaneously. This requires pharmacists to rely more on memory to correctly recall and associate information and observations with the correct patient

- Need for clinician input. Confirming details or consulting with physicians who are difficult to reach can delay work, increase production pressure, and contribute to interruptions.

- Alternating communication modes. Switching frequently between phone-based, paperbased, EHR-based, email, and in-person tasks and communication modes

- Excessive alerts and pop-ups. Encountering an extensive number of safety alerts and pop-ups might drive users to seek workarounds and ignore pop-ups

- Developing alarm fatigue. Developing alarm fatigue can lead to ignored or missed audible alarms, as well as a disdain for onscreen warnings

- Changing documentation modes. Shifting from documenting in paper charts to using a software-based solution might challenge familiar methods of documentation

- Lacking reference material. Lacking readily available reference material can make physicians rely on memory to recall procedures and instructions

- Interaction effect. Wearing protective equipment such as gloves can inhibit movement, visibility, and typing

\footnotetext{
${ }^{119}$ Adapted from https://www.accp.com/about/clinicalpharmacists.aspx
} 
- Stress of dealing with difficult patients or situations. Under stressful conditions, pharmacists might be more likely to take shortcuts, miss relevant cues, or to become frustrated when presented with obstacles to achieving their clinical goals while using the EHR

- Fatigue and Sleep Deprivation. Pharmacists often work long hours and can be working under the effects of acute and chronic sleep loss. This can lead to inattention, haste, a lower likelihood of detecting information presented in an EHR (thus suggesting a need to make critical information more salient), and a higher likelihood of ignoring prompts 


\section{References}

Altuwaijri, M. M. (2011, October). Achieving excellence in electronic health record deployment in Middle East hospitals. In Biomedical Engineering and Informatics (BMEI), 2011 4th International Conference (Vol. 4, pp. 1919-1923). IEEE.

Armijo, D., McDonnell, C., \& Werner, K. (2009). Electronic health record usability: evaluation and use case framework. AHRQ Publication, 9 (10).

Association for the Advancement of Medical Instrumentation. (2009). ANSI/AAMI HE75: 2009: Human factors engineering: Design of medical devices. Arlington, VA: Author.

Belden, J. L., Grayson, R., \& Barnes, J. (2009). Defining and testing EMR usability: Principles and proposed methods of EMR usability evaluation and rating. Healthcare Information and Management Systems Society (HIMSS).

Bevan, N. (2005, July). Guidelines and standards for web usability. In Proceedings of $\mathrm{HCl}$ International (Vol. 2005).

CFR (2012). Health information technology: Standards, implementation specifications, and certification criteria for Electronic Health Record technology, 2014 Edition. Code of Federal Regulations, 77(171).

Chen, Y., \& Lee, J. K. (2012, August). Analysis and evaluation about the barriers of the adoption and implementation of electronic health record system: A comparison study between China and Korea. In Information Technology in Medicine and Education (ITME), 2012 International Symposium on (Vol. 2, pp. 828-832). IEEE.

Christensen, C. (2012). A dashboard for OR patient safety optimization. Interview by Mark Hagland. Healthcare informatics: the business magazine for information and communication systems, 29(8), 29-31.

Connell, I. W. Full Principles Set. Set of 30 usability evaluation principles compiled by the author from the $\mathrm{HCl}$ literature, 2000.

Craig, D. (2011, May). An EHR interface for viewing and accessing patient health events from collaborative sources. In Collaboration Technologies and Systems (CTS), 2011 International Conference on (pp. 319-324). IEEE.

Davison, H. Cognitive task analysis: Current research [PowerPoint slides]. Retrieved from: http://web.mit.edu/16.459/www/CTA2.pdf.

DIS, I. (2009). 9241-210: 2010. Ergonomics of human system interaction-Part 210: Humancentered design for interactive systems. International Standardization Organization (ISO). Switzerland. 
Dringus, L.P. and Cohen, M.S. (2005). An adaptable usability heuristic checklist for online courses. $35^{\text {th }}$ ASEE/IEEE Frontiers in Education Conference.

Feblowitz, J. C., Wright, A., Singh, H., Samal, L., \& Sittig, D. F. (2011). Summarization of clinical information: A conceptual model. Journal of biomedical informatics, 44(4), 688-699.

Ferreira, A., Correia,R., and Antunes, M.B.L. (2012). Usable access control policy and model for healthcare. $24^{\text {th }}$ International Symposium on Computer-Based Medical Systems (CBMS).

Force, H. U. T. (2011). Promoting Usability in Health Organizations: initial steps and progress toward a healthcare usability maturity model. Health Information and Management Systems Society.

Ginsburg, M. (2012). Considering the visual element in clinical pediatric data management. 45th Hawaii International Conference on System Services.

Gocsik, T. (2012). Last man standing? Advice for engaging anesthesia clinicians when implementing an EMR in anesthesiology services. Healthcare informatics: the business magazine for information and communication systems, 29(10), 32-37.

Hagland, M. (2012). Physician documentation: Balancing clinical and administrative uses. Healthcare Informatics, 29(10), 30.

Horsky, J., Schiff, G. D., Johnston, D., Mercincavage, L., Bell, D., \& Middleton, B. (2012). Interface design principles for usable decision support: a targeted review of best practices for clinical prescribing interventions. Journal of biomedical informatics, 45(6), 1202-1216.

Ilie, V., Turel, O., \& Witman, P. D. (2013, January). Towards a New Design Paradigm for Complex Electronic Medical Record Systems: Intuitive User Interfaces. In System Sciences (HICSS), 2013 46th Hawaii International Conference (pp. 2585-2594). IEEE.

Kannampallil, T.G., Franklin, A., Mishra, R., Almosa, K.F., Cohen, T., and Patel, V.L. (2013). Understanding the nature of information seeking behavior in critical care: Implications for the design of health information technology. Artificial Intelligence in Medicine, 57(2013), 21-29.

Kannoju, P. K., Sridhar, K. Y., \& Prasad, P. S. (2010, November). A new paradigm of Electronic Health record for efficient implementation of healthcare delivery. In Biomedical Engineering and Sciences (IECBES), 2010 IEEE EMBS Conference (pp. 352-354). IEEE.

Katehakis, D. G., Sfakianakis, S. G., Kavlentakis, G., Anthoulakis, D. N., \& Tsiknakis, M. (2007). Delivering a lifelong integrated electronic health record based on a service oriented architecture. Information Technology in Biomedicine: IEEE Transactions, 11(6), 639-650.

Kaye, R., \& Crowley, J. (2000). Medical device use-safety: incorporating human factors engineering into risk management. Food and Drug Administration. 
Kimura, E., Kobayashi, S., Yoshikawa, T., and Ishihara, K. (2011). A framework for an authorization system with spatial reasoning capacity to improve risk management and information security in healthcare. IEEE/IPSJ International Symposium on Applications and the Internet.

Kobus, A. M., Harman, J. S., Do, H. D., \& Garvin, R. D. (2013). Challenges to depression care documentation in an EHR. Fam Med, 45(4), 268-71.

Koppel, R., Metlay, J. P., Cohen, A., Abaluck, B., Localio, A. R., Kimmel, S. E., \& Strom, B. L. (2005). Role of computerized physician order entry systems in facilitating medication errors. Jama, 293(10), 1197-1203.

Kume, N., Hirayama, Y., Ohboshi, N., Okamoto, K., Takemura, T., Araki, K., and Yoshihara (2012). The mobile environment of EHR browsing verified on tablet terminal. SCIS-ISIS.

Kuqi, K., Eveleigh, T., Holzer, T., \& Sarkani, S. (2012, March). Using design structure matrix for improving electronic medical record usability. In Systems Conference (SysCon), 2012 IEEE International (pp. 1-6). IEEE.

Kwak, Y. S. (2005, June). International standards for building electronic health record (ehr). In Enterprise networking and Computing in Healthcare Industry, 2005. HEALTHCOM 2005. In Proceedings of 7th International Workshop (pp. 18-23). IEEE.

Linder, J. A., Schnipper, J. L., Tsurikova, R., Melnikas, A. J., Volk, L. A., \& Middleton, B. (2006). Barriers to electronic health record use during patient visits. In AMIA Annual Symposium Proceedings (Vol. 2006, p. 499). American Medical Informatics Association.

Loranger, H., Schade, A., and Nielsen, J. (2002). Website Tools and Applications with Flash. Retrieved from: http://www.nngroup.com/reports/website-tools-and-applications-flash/

Militello, L. G., \& Hutton, R. J. (1998). Applied Cognitive Task Analysis (ACTA): A practitioner's toolkit for understanding cognitive task demands. Ergonomics, 41(11), 1618-1641.

Mosier, J. N., \& Smith, S. L. (1986). Application of guidelines for designing user interface software. Behaviour \& information technology, 5(1), 39-46.

Nielsen, J. (2005). Ten usability heuristics.

NISTIR 7804 (2012). Technical evaluation, testing and validation of the usability of Electronic Health Records. National Institute of Standards and Technology.

NISTIR 7865 (2012). A human factors guide to enhance EHR usability of critical user interactions when supporting pediatric patient care. National Institute of Standards and Technology.

NISTIR 7952 (2013). Toward a shared approach for ensuring patient safety with enhanced workflow design for Electronic Health Records: Workshop summary. National Institute of Standards and Technology. 
Patel, V. L., Zhang, J., Yoskowitz, N. A., Green, R., \& Sayan, O. R. (2008). Translational cognition for decision support in critical care environments: a review. Journal of biomedical informatics, 41(3), 413-431.

Perna, G. (2012). Mobility in nursing: An ongoing evolution. Why providers are investing strategically in nurse-friendly mobile solutions. Healthcare informatics: the business magazine for information and communication systems, 29(7), 16.

Prestigiacomo, J. (2011). Integrating telehealth and the EHR. Organizations make progress remotely linking physicians and patients, but documenting the remote consult will require much integration work. Healthcare informatics: the business magazine for information and communication systems, 29(6), 60-62.

Ratwani, R. M., Fairbanks, R. J., Hettinger, A. Z., \& Benda, N. C. (2015). Electronic health record usability: Analysis of the user-centered design processes of eleven electronic health record vendors. Journal of the American Medical Informatics Association, ocv050.

Ribitzky, R., Sterling, M., and Bradley, V. (2009). EHR usability pain points survey Q4-2009. HIMSS10: Annual Conference and Exhibition.

Rogoski, R. R. (2012). Bridging the EHR divide. Hospitals, physician practices work toward true interoperability. Healthcare informatics: the business magazine for information and communication systems, 29(10), 8-10.

Schiff, G. D., \& Bates, D. W. (2010). Can electronic clinical documentation help prevent diagnostic errors?. New England Journal of Medicine, 362(12), 1066-1069.

Schumacher, R. M., \& Lowry, S. Z. (2010). NIST guide to the processes approach for improving the usability of electronic health records. National Institute of Standards and Technology.

Schumacher, R. M., \& Lowry, S. Z. (2011). NISTIR 7742. Customized Common Industry Format Template for Electronic Health Record Usability Testing.

Shneiderman, B., \& Plaisant, C. (2005). Designing the user interface: strategies for effective. Human-Computer Interaction.

Sinha, P. K., Sunder, G., Bendale, P., Mantri, M., \& Dande, A. (2012). Electronic health record: standards, coding systems, frameworks, and infrastructures. John Wiley \& Sons.

Smith, S. L., \& Mosier, J. N. (1986). Guidelines for designing user interface software. Bedford, MA: Mitre Corporation.

Staggers, N. (2009). A systematic review on the designs of clinical technology: Findings and recommendations for future research. ANS. Advances in nursing science, 32(3), 252.

System Usability Scale (SUS). Retrieved from: http://www.usability.gov/how-to-andtools/methods/system-usability-scale.html 
Tancer, J., \& Varde, A. S. (2012, September). Cloud technology and EHR data management. In Information and Automation for Sustainability (ICIAfS), 2012 IEEE 6th International Conference on (pp. 112-117). IEEE.

Tarkan, S. (2013). Design Guidelines. Retrieved

from: http://www.cs.umd.edu/hcil/sharp/resultsmanagement/mstart/guidelines.pdf.

Tarkan, S., Franklin, L., Plaisant, C., \& Shneiderman, B. (2012). Design Guidelines for Ensuring Timely Management of Medical Orders. HCIL, UMD, Tech. Rep, 25.

Tractinsky, N., Katz, A. S., \& Ikar, D. (2000). What is beautiful is usable. Interacting with computers, 13(2), 127-145.

Wang, T. D., Wongsuphasawat, K., Plaisant, C., \& Shneiderman, B. (2010, November). Visual information seeking in multiple electronic health records: design recommendations and a process model. In Proceedings of the 1st ACM International Health Informatics Symposium (pp. 46-55). ACM.

Zhang, J., \& Walji, M. F. (2011). TURF: Toward a unified framework of EHR usability. Journal of biomedical informatics, 44(6), 1056-1067.

Zhang, J., Johnson, T. R., Patel, V. L., Paige, D. L., \& Kubose, T. (2003). Using usability heuristics to evaluate patient safety of medical devices. Journal of biomedical informatics, 36(1), 23-30. 